\title{
Outflows, infall and evolution of a sample of embedded low-mass protostars
}

\author{
The William Herschel Line Legacy (WILL) survey ${ }^{\star}$
}

\author{
J. C. Mottram ${ }^{1,2, \star \star}$, E. F. van Dishoeck ${ }^{1,3}$, L. E. Kristensen ${ }^{4,5}$, A. Karska ${ }^{3,6}$, I. San José-García ${ }^{1}$, S. Khanna ${ }^{1}$, \\ G. J. Herczeg ${ }^{7}$, Ph. André ${ }^{8}$, S. Bontemps ${ }^{9,10}$, S. Cabrit ${ }^{11,12}$, M. T. Carney ${ }^{1}$, M. N. Drozdovskaya ${ }^{1}$, M. M. Dunham ${ }^{4}$, \\ N. J. Evans ${ }^{13}$, D. Fedele ${ }^{2,14}$, J. D. Green ${ }^{13,15}$, D. Harsono ${ }^{1,16}$, D. Johnstone ${ }^{17,18}$, J. K. Jørgensen ${ }^{5}$, V. Könyves ${ }^{8}$, \\ B. Nisini ${ }^{19}$, M. V. Persson ${ }^{1}$, M. Tafalla ${ }^{20}$, R. Visser ${ }^{21}$, and U. A. Yıldiz ${ }^{22}$
}

(Affiliations can be found after the references)

Received 9 April 2016 / Accepted 17 November 2016

\begin{abstract}
Context. Herschel observations of water and highly excited $\mathrm{CO}(J>9)$ have allowed the physical and chemical conditions in the more active parts of protostellar outflows to be quantified in detail for the first time. However, to date, the studied samples of Class $0 / \mathrm{I}$ protostars in nearby star-forming regions have been selected from bright, well-known sources and have not been large enough for statistically significant trends to be firmly established.

Aims. We aim to explore the relationships between the outflow, envelope and physical properties of a flux-limited sample of embedded low-mass Class 0/I protostars.

Methods. We present spectroscopic observations in $\mathrm{H}_{2} \mathrm{O}, \mathrm{CO}$ and related species with Herschel $\mathrm{HIFI}$ and PACS, as well as ground-based follow-up with the JCMT and APEX in CO, $\mathrm{HCO}^{+}$and isotopologues, of a sample of 49 nearby $(d<500 \mathrm{pc})$ candidate protostars selected from Spitzer and Herschel photometric surveys of the Gould Belt. This more than doubles the sample of sources observed by the WISH and DIGIT surveys. These data are used to study the outflow and envelope properties of these sources. We also compile their continuum spectral energy distributions (SEDs) from the near-IR to $\mathrm{mm}$ wavelengths in order to constrain their physical properties (e.g. $L_{\mathrm{bol}}, T_{\mathrm{bol}}$ and $M_{\mathrm{env}}$ ).

Results. Water emission is dominated by shocks associated with the outflow, rather than the cooler, slower entrained outflowing gas probed by ground-based $\mathrm{CO}$ observations. These shocks become less energetic as sources evolve from Class 0 to Class I. Outflow force, measured from low- $J$ $\mathrm{CO}$, also decreases with source evolutionary stage, while the fraction of mass in the outflow relative to the total envelope (i.e. $M_{\text {out }} / M_{\text {env }}$ ) remains broadly constant between Class 0 and I. The median value of $\sim 1 \%$ is consistent with a core to star formation efficiency on the order of $50 \%$ and an outflow duty cycle on the order of $5 \%$. Entrainment efficiency, as probed by $F_{\mathrm{CO}} / \dot{M}_{\text {acc }}$, is also invariant with source properties and evolutionary stage. The median value implies a velocity at the wind launching radius of $6.3 \mathrm{~km} \mathrm{~s}^{-1}$, which in turn suggests an entrainment efficiency of between 30 and $60 \%$ if the wind is launched at $\sim 1 \mathrm{AU}$, or close to $100 \%$ if launched further out. $L\left[\mathrm{OI}\right.$ i is strongly correlated with $L_{\text {bol }}$ but not with $M_{\text {env }}$, in contrast to low- $J \mathrm{CO}$, which is more closely correlated with the latter than the former. This suggests that [O I] traces the present-day accretion activity of the source while $\mathrm{CO}$ traces time-averaged accretion over the dynamical timescale of the outflow. $\mathrm{H}_{2} \mathrm{O}$ is more strongly correlated with $M_{\text {env }}$ than $L_{\text {bol }}$, but the difference is smaller than low- $J C O$, consistent with water emission primarily tracing actively shocked material between the wind, traced by $[\mathrm{OI}]$, and the entrained molecular outflow, traced by low- $J \mathrm{CO} . L[\mathrm{OI}]$ does not vary from Class 0 to Class I, unlike $\mathrm{CO}$ and $\mathrm{H}_{2} \mathrm{O}$. This is likely due to the ratio of atomic to molecular gas in the wind increasing as the source evolves, balancing out the decrease in mass accretion rate. Infall signatures are detected in $\mathrm{HCO}^{+}$and $\mathrm{H}_{2} \mathrm{O}$ in a few sources, but still remain surprisingly illusive in single-dish observations.
\end{abstract}

Key words. stars: formation - stars: protostars - ISM: jets and outflows - surveys

\section{Introduction}

The general, cartoon picture of how stars form has been agreed for some time: a dense core within a molecular cloud becomes gravitationally unstable, causing material to fall inwards towards the centre; a protostar forms and launches a bi-polar molecular outflow; over time the outflow and infall combine to remove the envelope, eventually starving the protostar, which then slowly settles to the main sequence (e.g. Shu et al. 1987). However, a more detailed understanding is still required, particularly on infall and outflow, in order to quantitatively track the conversion of matter into stars and accurately predict the evolution and

\footnotetext{
* Herschel is an ESA space observatory with science instruments provided by European-led Principal Investigator consortia and with important participation from NASA.

$\star \star$ Corresponding author: J. C. Mottram, e-mail: mottram@mpia.de
}

outcome of the star-formation process for individual sources, stellar clusters and even whole galaxies.

The first step is improved quantification of the basic physical properties (e.g. $L_{\mathrm{bol}}, M_{\mathrm{env}}$ ) and evolutionary state of lowmass protostars, on which considerable progress has been made. Improvements in detectors and telescopes have lead to fullwavelength coverage from optical to radio wavelengths at better sensitivity and resolution, while dedicated very long baseline interferometry (VLBI) campaigns in the radio are providing much more accurate distances for nearby star-forming regions (e.g. Loinard 2013, for a recent review).

A framework for defining the evolutionary status of protostars has also been developed, dividing protostellar sources into one of five categories (Class 0, Class I, Flat, Class II and Class III) using various ways of quantifying the shift in the spectral energy distribution (SED) to shorter wavelengths 
as the source evolves: the infrared spectral index $\left(\alpha_{\mathrm{IR}}\right.$, e.g. Lada \& Wilking 1984; Lada 1987; Greene et al. 1994); the submillimetre $(\lambda>350 \mu \mathrm{m})$ to bolometric luminosity ratio $\left(L_{\text {submm }} / L_{\text {bol }}\right.$ used as a proxy for $M_{\text {env }} / L_{\text {bol }}$, e.g. André et al. 1993); and bolometric temperature $\left(T_{\text {bol }}\right.$, e.g. Myers \& Ladd 1993; Chen et al. 1995). For this latter measure, which is the intensity-weighted peak of the SED, these classifications are defined as: Class $0\left(T_{\text {bol }}<70 \mathrm{~K}\right)$, Class I $\left(70 \leq T_{\text {bol }}<650 \mathrm{~K}\right)$, Class II $\left(650 \leq T_{\text {bol }}<2800 \mathrm{~K}\right)$ and Class III $\left(T_{\text {bol }} \geq 2800 \mathrm{~K}\right)$. Flat-SED sources have $T_{\text {bol }}$ values in the $350-950 \mathrm{~K}$ range with a mean around $650 \mathrm{~K}$ (Evans et al. 2009).

The Spitzer Space Telescope (Gallagher et al. 2003) and more recently the Herschel Space Observatory (Pilbratt et al. 2010) have allowed the full potential of this evolutionary framework to be exploited in constraining how the properties of protostars change as the source evolves through large-area, high spatial resolution, uniform photometric surveys of many nearby star-forming regions (e.g. Evans et al. 2003, 2009; André et al. 2010; Rebull et al. 2010; Megeath et al. 2012; Dunham et al. 2014; Furlan et al. 2016). Furthermore, the statistics available from such large surveys have enabled estimates of the relative lifetimes of the different Classes to be obtained, showing in particular that the combined Class 0 and I phases, where the majority of the protostellar mass is accreted and the final properties of the star and its accompanying disk are imprinted, last approximately 0.4-0.7 Myr (Dunham et al. 2015; Heiderman \& Evans 2015; Carney et al. 2016).

For a $1 M_{\odot}$ star, such lifetimes imply typical timeaveraged mass-accretion rates onto the protostar of approximately $10^{-6} M_{\odot} \mathrm{yr}^{-1}$. Since not all material in the core will end up on the star, the infall rate in the envelope must presumably be higher than this by at least a factor of 2 or 3 . Searches to quantify the infall in protostars have presented candidates using molecular line observations (e.g. Gregersen et al. 1997; Mardones et al. 1997) based on the doppler-shift of infalling material causing asymmetries in the line profile (Myers et al. 2000). However, confirming and quantifying infall in protostellar envelopes remains extremely challenging, limiting our understanding of the rate at which, and route by which, material reaches the disk and protostar, as well as how this changes with time and depends on the mass of the core/star.

Bipolar molecular outflows also play an important role in the evolution and outcome of star formation, as they remove mass from and inject energy into the envelope and surrounding material. However, the driving mechanism for protostellar outflows is still uncertain (e.g. Arce et al. 2007; Frank et al. 2014). A decrease in the driving force was measured between Class 0 and I sources, in addition to relations with $L_{\mathrm{bol}}$ and $M_{\mathrm{env}}$, by Bontemps et al. (1996) using ground-based observations of CO. They attributed the decrease in outflow driving force with Class to a decrease in the accretion/infall rate as the source evolves. However, their study only included ten Class 0 sources, as few were known at the time.

Recent observations of $\mathrm{H}_{2} \mathrm{O}$ and highly-excited $\mathrm{CO}$ using the Heterodyne Instrument for the Far-Infrared (HIFI; de Graauw et al. 2010) and Photodetector Array Camera and Spectrometer (PACS; Poglitsch et al. 2010) with Herschel have shown that these primarily trace active shocks related to the outflow and/or warm disk winds heated by ambipolar diffusion, rather than the entrained outflow as is accessible with ground-based CO observations (Nisini et al. 2010; Kristensen et al. 2013; Tafalla et al. 2013; Santangelo et al. 2013, 2014; Mottram et al. 2014; Yvart et al. 2016). The linewidth and intensity in these tracers decreases between Class 0 and I while the excitation conditions $(T, N, n)$ remain the same (Mottram et al. 2014; Manoj et al. 2013; Karska et al. 2013, 2014a; Green et al. 2013a; Matuszak et al. 2015). However, these studies have typically considered relatively small samples $(N \lesssim 30)$ of bright, well-known sources and so the statistical significance of trends with evolution and other source parameters has, in some cases, been low.

Two of the main surveys studying nearby Class 0/I protostars with Herschel were the "Water in star-forming regions with Herschel" (WISH) guaranteed time key program (van Dishoeck et al. 2011), which observed 29 Class 0/I protostars with HIFI and PACS plus ground-based follow-up, and the "Dust, Ice, and Gas in Time" (DIGIT) Herschel key program (Green et al. 2013a, 2016), which observed a further 13 Class 0/I protostars, primarily with full-scan PACS spectroscopy. Both the WISH and DIGIT surveys selected their samples to target well known, archetypal sources, ensuring success in detecting water, $\mathrm{CO}$ and other species and the availability of complementary data. As a result, these samples favoured luminous sources with particularly prominent and extended outflows, which may not be representative of the general population of protostars. In addition, both programs together only included a total of 42 lowmass sources split between Classes 0 and I, limiting the statistical significance of trends with evolution that might otherwise have been expected, for example between integrated intensity in water emission and $T_{\text {bol }}$.

The motivation of the William Herschel Line Legacy (WILL) survey was therefore to further explore the physics (primarily infall and outflow) and chemistry of water, $\mathrm{CO}$ and other complementary species in Class $0 / \mathrm{I}$ protostars in nearby lowmass star forming regions using a combination of Herschel and ground-based observations, building on WISH and DIGIT. The aim was to increase the number of Class $0 /$ I protostars observed, thus improving the statistical significance of the existing correlations found by for example Kristensen et al. (2012), and allowing shallower correlations to be tested, as well as improving the sampling of fainter and colder sources.

This paper is structured as follows. Section 2 discusses the selection of the WILL sample, the basic physical properties of the sources and evaluates the properties of the combined WISH+DIGIT+WILL sample. Section 3 gives the details and basic results of both the Herschel observations and a complementary ground-based follow-up campaign. More detailed results and analysis are then presented thematically, centred around outflows (Sect. 4) and envelope emission (Sect. 5), followed by a discussion on the variation of water with evolution (Sect. 6). Finally, we summarise our main conclusions in Sect. 7.

\section{Sample}

\subsection{Selection}

The starting point for selecting a flux-limited sample of lowmass protostars was the catalogue of Class 0/I protostars identified as part of photometric surveys with the Spitzer Space Telescope of the closest major star-forming clouds that make up the Gould Belt (Gould 1879). In particular, these were drawn from the Spitzer c2d (Evans et al. 2009), Spitzer Gould Belt (Dunham et al. 2015) and Taurus Spitzer (Rebull et al. 2010) surveys.

The initial catalogue was compiled from individual cloud catalogues for the Perseus, Taurus, Ophiuchus, Scorpius (also known as Ophiuchus North), Corona Australis and Chameleon 
star-forming regions (for more details, see Jørgensen et al. 2007; Rebull et al. 2007, 2010; Padgett et al. 2008; Jørgensen et al. 2008; Hatchell et al. 2012; Peterson et al. 2011; Alcalá et al. 2008). At the time of selection in 2011, the Herschel Gould Belt (André et al. 2010) survey had also produced catalogues of protostellar candidates in the Aquila Rift region (Maury et al. 2011), so these were also considered in an attempt to extend the coverage of the WILL survey to particularly young (cold) embedded young stellar objects (YSOs).

From this master catalogue of protostars in major starforming regions within $500 \mathrm{pc}$, the following criteria were used to select the final WILL sample:

(i) infrared slope $(2-24 \mu \mathrm{m}) \alpha_{\mathrm{IR}}>0.3$ or non-detection;

(ii) $T_{\text {bol }}<350 \mathrm{~K}$

(iii) $L_{\mathrm{bol}}>0.4 L_{\odot}$ for Class $0\left(T_{\mathrm{bol}}<70 \mathrm{~K}\right)$, $L_{\mathrm{bol}} \geq 1 L_{\odot}$ for Class I $\left(70 \leq T_{\mathrm{bol}}<350 \mathrm{~K}\right)$;

(iv) $\delta<35^{\circ}$.

The distinction between Class I and II sources is normally made at $T_{\text {bol }}=650 \mathrm{~K}$ (Chen et al. 1995), however Evans et al. (2009) found that Flat SED sources cover the range 350-950 K with a mean around $650 \mathrm{~K}$ and therefore likely consist of more evolved Class I or younger Class II sources. An upper limit of $350 \mathrm{~K}$ was therefore imposed in order to exclude more evolved Class I sources from the sample. Water emission is typically weaker for Class I sources than Class 0s and is generally higher for more luminous sources (e.g. Kristensen et al. 2012), so a higher $L_{\text {bol }}$ cut was used for Class I sources in an attempt to ensure detections. Criteria i-iii were therefore designed to ensure that the sample includes only young, deeply embedded protostars that are bright enough to be detected in $\mathrm{H}_{2} \mathrm{O}$ and related species based on the experience of the WISH and DIGIT surveys. Criterion iv ensures that all WILL sources can be observed with ALMA to allow high spectral and spatial resolution ground-based interferometric follow-up of interesting sources.

Unfortunately, edge-on disks, reddened background sources and evolved asymptotic giant-branch (AGB) stars all have the potential to present similar infrared colours and thus contaminate any sample selected purely based on continuum properties. As first highlighted by van Kempen et al. (2009) for a sample of sources in Ophiuchus, molecular emission tracing dense gas can help to break this degeneracy. More specifically, the high critical density of $\mathrm{HCO}^{+} J=4-3$ or $J=3-2$ means it will not be strong in foreground cloud material, while the rarity of $\mathrm{C}^{18} \mathrm{O}$ similarly means that the $J=3-2$ transition is only bright and concentrated in protostellar sources. In addition, more evolved disk sources will not present strong emission in single-dish $\mathrm{HCO}^{+}$spectra due to beam-dilution. Such data, particularly for $\mathrm{HCO}^{+}$, have been collected and used to remove contaminants in a number of Gould Belt samples by Heiderman et al. (2010), Heiderman \& Evans (2015) and Carney et al. (2016), which have some overlap with the initial candidate sample. Therefore, following the cuts detailed above, non-detection in $\mathrm{HCO}^{+} J=4-3$ or 3-2 was used, where data were available, to exclude contaminant sources.

Most of the sources observed by the WISH and DIGIT surveys also conform to the above criteria, so any initial candidates within $5^{\prime \prime}$ of a WISH or DIGIT source were also excluded to avoid repeat observations. However, two sources, PER 03 and PER 11, have enough overlap with the WISH observations of L1448-MM (offset by 7.7") and NGC 1333-IRAS4B (offset by $\left.6.4^{\prime \prime}\right)$, respectively, particularly in the $\mathrm{H}_{2} \mathrm{O} 1_{10}-1_{01}(557 \mathrm{GHz})$ ground-state line obtained in a $39^{\prime \prime}$ beam, that they are removed from the WILL sample as presented here. Finally, source
TAU 05 was removed as it is the young and active Class II source DG Tau B, which has an edge-on disk (Podio et al. 2013).

\subsection{Properties and evaluation}

The properties of the final sample of 49 sources that make up the WILL sample are presented in Table 1. For simplicity, we give each a name based on the region and a number ordered by right ascension, but many are already well known and therefore the table also gives details of common names used by previous studies for the same sources.

The following distances are used for the various regions covered by our sample: $235 \mathrm{pc}$ for Perseus (Hirota et al. 2008), $140 \mathrm{pc}$ for Taurus (Kenyon et al. 2008), $125 \mathrm{pc}$ for Ophiuchus and Scorpius (de Geus et al. 1989), 130 pc for Corona Australis (Knude \& Høg 1998), 150 pc for Chameleon I and 178 pc for Chameleon II (Whittet et al. 1997). For Aquila, W40 and Serpens South, Ortiz-León et al. (2017) recently found that these regions, as well as Serpens Main, are at a common distance of $436 \mathrm{pc}$.

The determination of the source properties and evolutionary classification is discussed in detail in Appendix A. To summarise briefly, the SED for each source is constructed from the near-IR to (sub-)mm and used to calculate $L_{\text {bol }}, L_{\text {submm }} / L_{\text {bol }}, T_{\text {bol }}$ and $\alpha_{\mathrm{IR}}$. $M_{\text {env }}$ is obtained from sub-mm or mm photometry assuming that the dust is optically thin, while $v_{\mathrm{LSR}}$ is calculated from molecular line observations. Finally, the classification of each source is reached by considering the spatial and spectral properties of both the gas and dust associated with each source (see Appendix A.7 for more details).

The sample comprises 23 Class 0, 14 Class I, 8 Class II and 4 uncertain, potentially pre-stellar sources. In the case of this last group of sources, all in W40, they are faint or not detected at $<160 \mu \mathrm{m}$, show few detections in PACS and have no indications of outflow activity, but the presence of the W40 PDR, detected in some of the HIFI and ground-based lines, leaves some ambiguity. These and other cases of note are discussed in more detail in Appendix C.

Figure 1 shows the $L_{\mathrm{bol}}, T_{\mathrm{bol}}$ and $M_{\mathrm{env}}$ distribution of the WILL sample, along with the WISH and DIGIT samples for comparison. The properties of the WISH sample are taken from Kristensen et al. (2012) while those for the DIGIT sample are taken from Green et al. (2013a) and Lindberg et al. (2014). These are corrected to the distances for the various regions discussed above where needed. It should be noted that $M_{\text {env }}$ values are not available for the DIGIT sample, leading to the difference in the number of sources between the upper-left and upper-right panels.

The probability (p) that a given value of the Pearson correlation coefficient $(\rho)$ for sample size $n$ represents a real correlation (i.e. the likelihood that a two-tailed test can reject the nullhypothesis that the two variables are uncorrelated with $\rho=0$ ) can be expressed in terms of the standard deviation of a normal distribution, $\sigma$, as:

$p=|\rho| \sqrt{n-1} \sigma$,

following (Marseille et al. 2010). We consider $p=3 \sigma$ (i.e. $99.7 \%$ ) to be the threshold for statistical significance. Thus, for a sample size of 30 , values of $|\rho|>0.56$ indicate real, statistically significant correlations while for a sample size of 50, this is true for $|\rho|>0.43$. While one might expect correlations between some of the observed properties of embedded protostars due to the related nature of their different components (e.g. envelope, 
Table 1. The WILL survey source sample.

\begin{tabular}{|c|c|c|c|c|c|c|c|c|c|c|c|}
\hline Name & $\begin{array}{l}\text { RA }(J 2000) \\
(\mathrm{h} \mathrm{m} \mathrm{s})\end{array}$ & $\begin{array}{c}\operatorname{Dec}(J 2000) \\
\left({ }^{\circ}, \prime \prime\right)\end{array}$ & $\begin{array}{c}d \\
(\mathrm{pc})\end{array}$ & $\begin{array}{c}v_{\mathrm{LSR}}{ }^{a} \\
\left(\mathrm{~km} \mathrm{~s}^{-1}\right) \\
\end{array}$ & $\begin{array}{l}L_{\mathrm{bol}}^{b} \\
\left(L_{\odot}\right) \\
\end{array}$ & $\begin{array}{c}\frac{L_{\mathrm{submm}}}{} b \\
L_{\mathrm{bol}} \\
(\%)\end{array}$ & $\begin{array}{r}T_{\mathrm{bol}}{ }^{b} \\
(\mathrm{~K}) \\
\end{array}$ & $\alpha_{\mathrm{IR}}^{b}$ & $\begin{array}{c}M_{\mathrm{env}}{ }^{b} \\
\left(M_{\odot}\right)\end{array}$ & Class $^{c}$ & Other names $^{d}$ \\
\hline $\mathrm{AQU} 01^{e}$ & $18: 29: 03.82$ & $-01: 39: 01.5$ & 436 & +7.4 & 2.6 & 11.8 & 24 & - & 3.15 & 0 & Aqu-MM2 \\
\hline $\mathrm{AQU} 02^{e}$ & $18: 29: 08.60$ & $-01: 30: 42.8$ & 436 & +7.5 & 9.0 & 7.8 & 33 & - & 2.17 & 0 & Aqu-MM4, IRAS 18265-0132 \\
\hline $\mathrm{AQU} 03^{e}$ & $18: 30: 25.10$ & $-01: 54: 13.4$ & 436 & +7.1 & 3.5 & 5.3 & 246 & 0.7 & 0.79 & II & Aqu-MM6, IRAS 18278-0156 \\
\hline AQU $04^{e}$ & 18:30:28.63 & $-01: 56: 47.7$ & 436 & +7.6 & 6.5 & 4.5 & 320 & 0.5 & 1.21 & I & Aqu-MM7, IRAS 18278-0158 \\
\hline AQU 05 & $18: 30: 29.03$ & $-01: 56: 05.4$ & 436 & +7.3 & 2.4 & 9.2 & 37 & 1.4 & 0.68 & 0 & Aqu-MM10 \\
\hline AQU 06 & 18:30:49.94 & $-01: 56: 06.1$ & 436 & +8.3 & 1.3 & 8.2 & 40 & 1.9 & 0.59 & 0 & Aqu-MM14 \\
\hline CHA 01 & 11:09:28.51 & $-76: 33: 28.4$ & 150 & +4.9 & 1.6 & - & 189 & 1.6 & - & II & GM Cha, ISO-ChaI 192, CaINa2 \\
\hline CHA 02 & 12:59:06.58 & $-77: 07: 39.9$ & 178 & +3.0 & 1.8 & 0.6 & 236 & 1.3 & - & I & ISO-ChaII 28, IRAS 12553-7651 \\
\hline CRA 01 & 19:02:58.67 & $-37: 07: 35.9$ & 130 & +5.6 & 2.4 & 2.2 & 55 & 1.7 & 0.49 & 0 & ISO-CrA 182, IRAS 18595-3712 \\
\hline OPH 01 & $16: 26: 59.10$ & $-24: 35: 03.3$ & 125 & +3.8 & 4.3 & - & 69 & 2.0 & 0.17 & $\mathrm{II}+\mathrm{PDR} ?$ & ISO-Oph 90, WL 22 \\
\hline OPH 02 & $16: 32: 00.99$ & $-24: 56: 42.6$ & 125 & +4.2 & 8.6 & 0.1 & 80 & 1.8 & 0.09 & I & ISO-Oph 209, Oph-emb 10 \\
\hline PER 01 & $03: 25: 22.32$ & $+30: 45: 13.9$ & 235 & +4.1 & 4.5 & 2.7 & 44 & 2.3 & 0.89 & 0 & L1448 IRS2, Per-emb 22 \\
\hline PER 02 & 03:25:36.49 & $+30: 45: 22.2$ & 235 & +4.5 & 9.2 & 1.7 & 54 & 2.6 & 3.48 & 0 & L1448 N(A), L1448 IRS3, Per-emb 33 \\
\hline PER 04 & $03: 26: 37.47$ & $+30: 15: 28.1$ & 235 & +5.2 & 1.2 & 4.2 & 60 & 1.2 & 0.29 & 0 & IRAS 03235+3004, Per-emb 25 \\
\hline PER 05 & 03:28:37.09 & $+31: 13: 30.8$ & 235 & +7.3 & 11.1 & 0.6 & 84 & 2.2 & 0.36 & I & NGC 1333 IRAS1, Per-emb 35 \\
\hline PER 06 & 03:28:57.36 & $+31: 14: 15.9$ & 235 & +7.3 & 7.1 & - & 82 & 1.5 & 0.34 & I & NGC 1333 IRAS2B, Per-emb 36 \\
\hline PER 07 & 03:29:00.55 & $+31: 12: 00.8$ & 235 & +7.4 & 0.7 & 3.9 & 37 & 2.1 & 0.32 & 0 & Per-emb 3 \\
\hline PER 08 & 03:29:01.56 & $+31: 20: 20.6$ & 235 & +7.7 & 16.9 & 1.3 & 129 & 2.5 & 0.83 & I & Per-emb 54, NGC 1333 IRAS6 \\
\hline PER 09 & 03:29:07.78 & $+31: 21: 57.3$ & 235 & +7.5 & 22.7 & - & 129 & 2.6 & 0.26 & I & IRAS 03260+3111(W), Per-emb 50 \\
\hline PER 10 & 03:29:10.68 & $+31: 18: 20.6$ & 235 & +8.7 & 6.0 & 2.2 & 47 & 1.9 & 1.10 & 0 & NGC 1333 IRAS7, Per-emb 21 \\
\hline PER 12 & 03:29:13.54 & $+31: 13: 58.2$ & 235 & +7.8 & 1.1 & 8.7 & 31 & 2.4 & 1.20 & 0 & NGC 1333 IRAS4C, Per-emb 14 \\
\hline PER 13 & 03:29:51.82 & $+31: 39: 06.0$ & 235 & +8.0 & 0.7 & 5.0 & 40 & 3.5 & 0.49 & 0 & IRAS 03267+3128, Per-emb 9 \\
\hline PER 14 & 03:30:15.14 & $+30: 23: 49.4$ & 235 & +6.2 & 1.8 & 1.6 & 88 & 1.8 & 0.15 & I & IRAS 03271+3013, Per-emb 34 \\
\hline PER 15 & 03:31:20.98 & $+30: 45: 30.1$ & 235 & +6.9 & 1.6 & 5.8 & 36 & 1.2 & 1.16 & 0 & IRAS 03282+3035, Per-emb 5 \\
\hline PER 16 & 03:32:17.96 & $+30: 49: 47.5$ & 235 & +7.0 & 1.1 & 13.3 & 29 & 1.0 & 2.88 & 0 & IRAS 03292+3039, Per-emb 2 \\
\hline PER 17 & 03:33:14.38 & $+31: 07: 10.9$ & 235 & +6.6 & 0.2 & - & 71 & 2.4 & 1.94 & I & B1 SMM3, Per-emb 6 \\
\hline PER 18 & 03:33:16.44 & $+31: 06: 52.5$ & 235 & +6.6 & 0.5 & - & 38 & 1.6 & 1.59 & 0 & B1d, Per-emb 10 \\
\hline PER 19 & 03:33:27.29 & $+31: 07: 10.2$ & 235 & +6.8 & 1.1 & 1.7 & 93 & 1.9 & 0.29 & I & B1 SMM11, Per-emb 30 \\
\hline PER 20 & $03: 43: 56.52$ & $+32: 00: 52.8$ & 235 & +8.9 & 2.2 & 6.3 & 27 & 0.7 & 1.93 & 0 & IRAS $03407+3152$, HH 211, Per-emb 1 \\
\hline PER 21 & 03:43:56.84 & $+32: 03: 04.7$ & 235 & +8.8 & 1.9 & 3.8 & 35 & 1.5 & 1.54 & 0 & IC348 MMS, Per-emb 11 \\
\hline PER 22 & 03:44:43.96 & $+32: 01: 36.2$ & 235 & +9.8 & 2.4 & 3.4 & 45 & 0.9 & 0.70 & 0 & IRAS 03415+3152, Per-emb 8 \\
\hline SCO 01 & $16: 46: 58.27$ & $-09: 35: 19.8$ & 125 & $+3.6^{f}$ & 0.5 & 0.6 & 201 & 0.9 & 0.10 & II & IRAS 16442-0930, L260 SMM1 \\
\hline SERS 01 & $18: 29: 37.70$ & $-01: 50: 57.8$ & 436 & +8.2 & 17.4 & 3.9 & 46 & 1.3 & 1.10 & 0 & IRAS 18270-0153, SerpS-MM1 \\
\hline SERS 02 & $18: 30: 04.13$ & $-02: 03: 02.1$ & 436 & +7.8 & 73.2 & 4.6 & 34 & 2.5 & 8.44 & 0 & SerpS-MM18 \\
\hline TAU 01 & $04: 19: 58.40$ & $+27: 09: 57.0$ & 140 & +6.8 & 1.5 & 3.3 & 136 & 1.4 & 0.27 & I & IRAS 04169+2702 \\
\hline TAU 02 & $04: 21: 11.40$ & $+27: 01: 09.0$ & 140 & +6.6 & 0.5 & 0.8 & 282 & 0.5 & - & I & IRAS 04181+2654A \\
\hline TAU 03 & 04:22:00.60 & $+26: 57: 32.0$ & 140 & $+7.4^{f}$ & 0.4 & 0.2 & 196 & 1.0 & - & II & IRAS 04189+2650(W) \\
\hline TAU 04 & 04:27:02.60 & $+26: 05: 30.0$ & 140 & +6.3 & 1.4 & 1.5 & 161 & 0.8 & 0.64 & I & DG TAU B \\
\hline TAU 06 & 04:27:57.30 & $+26: 19: 18.0$ & 140 & +7.2 & 0.6 & 2.7 & 80 & 0.8 & 0.09 & I & HH31 IRS 2, IRAS 04248+2612 \\
\hline TAU 07 & 04:29:30.00 & $+24: 39: 55.0$ & 140 & $+6.3^{f}$ & 0.6 & 0.2 & 169 & 0.9 & - & II & HH 414, IRAS 04264+2433 \\
\hline TAU 08 & 04:32:32.00 & $+22: 57: 26.0$ & 140 & $+5.5^{g}$ & 0.5 & 1.2 & 300 & 0.5 & 0.18 & II & L1536 IRS, IRAS 04295+2251 \\
\hline TAU 09 & $04: 35: 35.30$ & $+24: 08: 19.0$ & 140 & +5.5 & 1.0 & 1.7 & 82 & 1.4 & 0.06 & II & L1535 IRS, IRAS 04325+2402 \\
\hline W40 01 & $18: 31: 09.42$ & $-02: 06: 24.5$ & 436 & +4.9 & 13.3 & 7.4 & 40 & 2.3 & 1.97 & $0+\mathrm{PDR}$ & W40-MM3 \\
\hline W40 02 & $18: 31: 10.36$ & $-02: 03: 50.4$ & 436 & +4.8 & 32.6 & 3.7 & 46 & 4.6 & 2.25 & 0 & W40-MM5 \\
\hline W40 03 & $18: 31: 46.54$ & $-02: 04: 22.5$ & 436 & +6.4 & 8.3 & 20.6 & 15 & - & 3.37 & PS?+PDR & W40-MM26 \\
\hline W40 04 & $18: 31: 46.78$ & $-02: 02: 19.9$ & 436 & +6.7 & 6.1 & 9.4 & 16 & - & 1.69 & PS?+PDR & W40-MM27 \\
\hline W40 05 & $18: 31: 47.90$ & $-02: 01: 37.2$ & 436 & +6.5 & 5.9 & 27.3 & 14 & - & 1.97 & PS?+PDR & W40-MM28 \\
\hline W40 06 & $18: 31: 57.24$ & $-02: 00: 27.7$ & 436 & +6.6 & 4.1 & 2.2 & 33 & - & 0.31 & PS?+PDR & W40-MM34 \\
\hline W40 07 & $18: 32: 13.36$ & $-01: 57: 29.6$ & 436 & +7.4 & 3.6 & 3.3 & 36 & 0.9 & 0.25 & 0 & W40-MM36 \\
\hline
\end{tabular}

Notes. ${ }^{(a)}$ From Gaussian fits to the $\mathrm{C}^{18} \mathrm{O} J=3-2$ observations (see Table A.9). ${ }^{(b)}$ Calculated as discussed in Sect. A.7. ${ }^{(c)}$ Evolutionary classification, see Sect. A.7 for details of the determination. PS = pre-stellar, PDR = narrow, bright ${ }^{12} \mathrm{CO} J=10-9$ emission consistent with a photon-dominated region. ${ }^{(d)}$ First additional names for Aquila, Serpens South and W40 are from Maury et al. (2011), “-emb" names from Enoch et al. (2009). ${ }^{(e)}$ Sources off-centre in beam. Peak coordinates in PACS maps used for extraction of ground-based data: AQU 01 18:29:03.61 -01:39:05.6; AQU 02 18:29:08.20 -01:30:46.6; AQU 03 18:30:24.69 - 01:54:11.0; AQU 04 18:30:29.32 -01:56:42.4. ${ }^{(f)}$ From Gaussian fits to the ${ }^{13} \mathrm{CO}(J=3-2)$ observations as $\mathrm{C}^{18} \mathrm{O}$ is not detected. ${ }^{(g)}$ Taken from Caselli et al. (2002).

outflow and driving source), such tests are a simple way of ascertaining whether or not the data are able to support such links. As mentioned above, the extension of the sample of sources studied in spectral lines with PACS and HIFI enabled by the WILL survey and presented here allows us to study these more completely for the first time.

The evolutionary tracks between $L_{\text {bol }}$ and $M_{\text {env }}$ shown in the top-right panel of Fig. 1 are taken from
Duarte-Cabral et al. (2013). They assume an exponential decrease of $M_{\text {env }}$ and a core-to-star formation efficiency of $50 \%$, such that the net accretion rate is given by:

$\dot{M}_{\mathrm{acc}}(t)=0.5 \frac{M_{\mathrm{env}}(t)}{\tau}$,

where $\tau$ is the e-folding time, which is assumed to be $3 \times 10^{5} \mathrm{yr}$. 

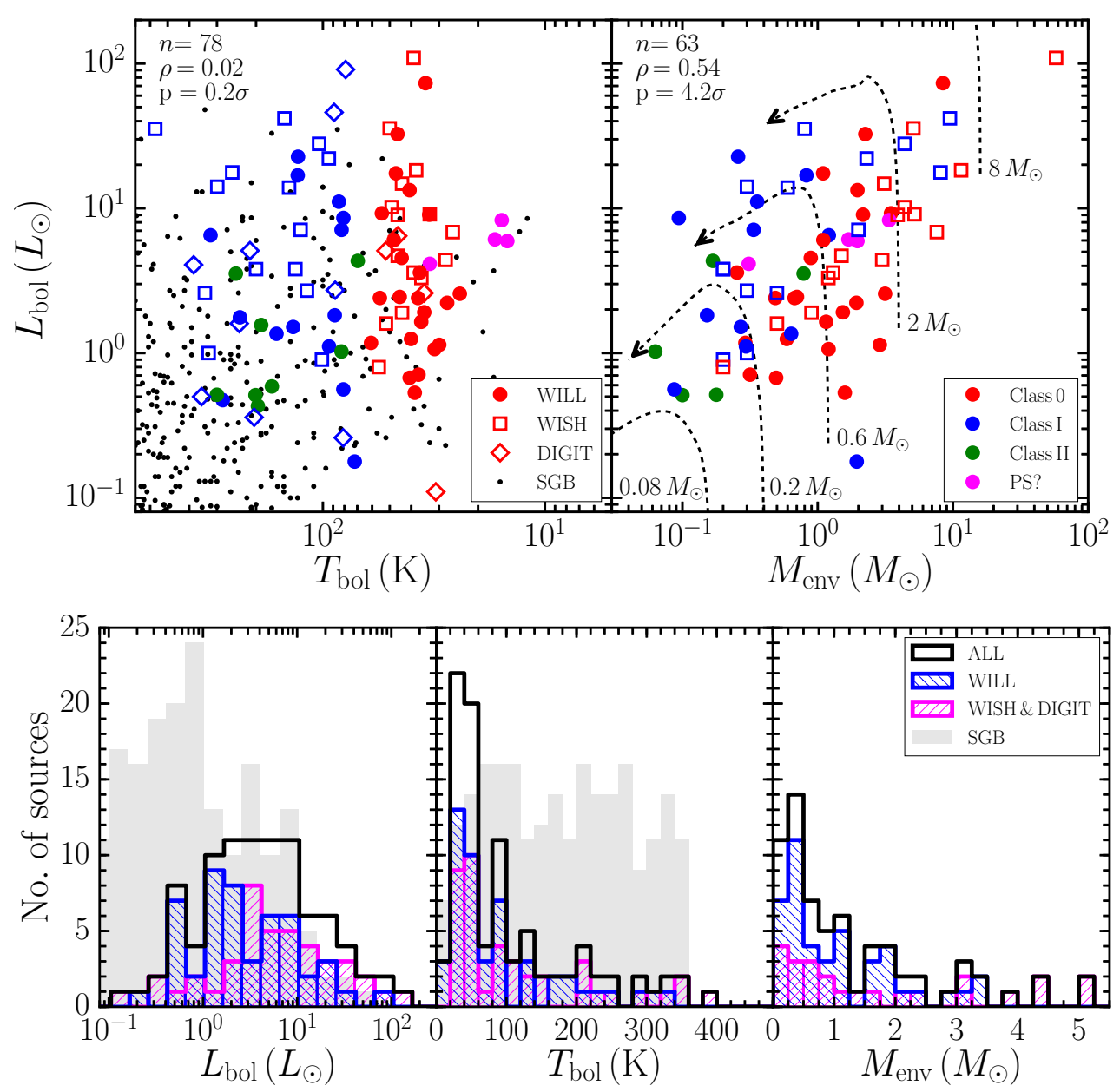

Fig. 1. Top: distribution of $L_{\mathrm{bol}}$ vs. $T_{\mathrm{bol}}$ and $M_{\mathrm{env}}$ for the WILL (filled circles), WISH (open squares) and DIGIT (open diamonds) surveys. In the left-hand panel, the Spitzer Gould Belt (SGB) determinations from Dunham et al. (2015) are shown for comparison (black dots). The different colours are used to distinguish between different source classifications: Class 0 (red), Class I (blue) Class II (green) and pre-stellar (PS, magenta). The number of sources $(n)$, Pearson correlation coefficient $(\rho)$, and the probability $(p)$ that the correlation is not just due to random distributions in the variables are shown in the upper-left of each panel including only Class 0/I sources. Evolutionary tracks between $L_{\mathrm{bol}}$ and $M_{\mathrm{env}}$ from Duarte-Cabral et al. (2013) are shown in the right-hand panel (see text for details), with the final stellar mass indicated for each track. Bottom: histograms showing the distribution of $L_{\mathrm{bol}}, T_{\mathrm{bol}}$ and $M_{\mathrm{env}}$ for the WILL (blue), combined WISH and DIGIT (magenta hatched), and total WILL, WISH and DIGIT (black) samples. The grey shaded region indicates the distribution of the Spitzer Gould Belt determinations for sources with $T_{\text {bol }} \leq 350 \mathrm{~K}$.

The WILL sample doubles the number of low-mass YSOs observed, which have slightly lower values of $L_{\mathrm{bol}}$ and $M_{\mathrm{env}}$, as well as lower $T_{\text {bol }}$ for Class 0 sources, than the WISH and DIGIT samples. Comparing to Spitzer Gould Belt (SGB) sources with $T_{\text {bol }} \leq 350 \mathrm{~K}$, taken from Dunham et al. (2015), it can be seen in Fig. 1 that the combined WILL+DIGIT+WISH sample is representative of the overall Class 0/I population and contains most sources above $\sim 1 L_{\odot}$. Below this luminosity, the sample rapidly becomes incomplete, and thus the combined sample is still biased towards higher mean $L_{\mathrm{bol}}$ compared with the general distribution, but the addition of the WILL sources shifts the completeness limit approximately a factor of three lower. In terms of $T_{\text {bol }}$, the sample is biased towards lower values, but judging from upper-left panel of Fig. 1, the higher $T_{\text {bol }}$ sources in the SGB data are primarily those below our $L_{\text {bol }}$ limit, that is, the mean $L_{\text {bol }}$ decreases as $T_{\text {bol }}$ increases for SGB sources. The differences between the values of Dunham et al. (2015) and those given here for individual sources are likely due to our inclusion of far-IR data in these determinations.

It is worth mentioning a couple of caveats. Firstly, the sample of Class 0 sources is dominated by sources in the Perseus molecular cloud, while the Class I sources are drawn from a number of regions that vary in the concentration and activity of their star formation (e.g. Taurus vs. Ophiuchus). There may well be regional differences due to environmental effects, which we cannot test due to the overall small sample size for a given region. Secondly, by excluding older Class I and flat-spectrum sources, we introduce a bias towards younger Class I sources, so the properties of an average Class I source may well be slightly different from those determined with this sample. However, in general for the part of parameter space that WILL, WISH and DIGIT are designed to probe, the addition of the WILL survey leaves the combined sample broadly complete.

\section{Observations and results}

The primary observations for the WILL survey were taken with Herschel ${ }^{1}$ using the Heterodyne Instrument for the Far-Infrared

\footnotetext{
1 Herschel is an ESA space observatory with science instruments provided by European-led Principal Investigator consortia and with important participation from NASA.
} 
Table 2. Principle lines observed with HIFI.

\begin{tabular}{|c|c|c|c|c|c|c|c|c|c|c|c|}
\hline Species & Transition & $\begin{array}{c}\text { Rest frequency }{ }^{a} \\
(\mathrm{GHz})\end{array}$ & $\begin{array}{l}E_{\mathrm{u}} / k_{\mathrm{b}} \\
(\mathrm{K})\end{array}$ & $\begin{array}{l}A_{\mathrm{ul}}^{b} \\
\left(\mathrm{~s}^{-1}\right)\end{array}$ & $\begin{array}{c}n_{\mathrm{cr}}{ }^{c} \\
\left(\mathrm{~cm}^{-3}\right)\end{array}$ & $\begin{array}{l}\eta_{\mathrm{mb}}{ }^{d} \\
(\mathrm{H} / \mathrm{V})\end{array}$ & $\begin{array}{c}\theta_{\mathrm{mb}}^{e} \\
\left({ }^{\prime \prime}\right)\end{array}$ & $\begin{array}{l}\text { WBS resolution } \\
\quad\left(\mathrm{km} \mathrm{s}^{-1}\right)\end{array}$ & $\begin{array}{l}\text { HRS resolution } \\
\quad\left(\mathrm{km} \mathrm{s}^{-1}\right)\end{array}$ & $\begin{array}{l}\text { Obs. time }{ }^{f} \\
\quad(\min )\end{array}$ & $\operatorname{Det}^{g}$ \\
\hline $\mathrm{o}-\mathrm{H}_{2} \mathrm{O}$ & $\begin{array}{l}1_{10}-1_{01} \\
3_{12}-2_{21}\end{array}$ & $\begin{array}{r}556.93599 \\
1153.12682\end{array}$ & $\begin{array}{r}61.0 \\
249.4\end{array}$ & $\begin{array}{l}3.46 \times 10^{-3} \\
2.63 \times 10^{-3}\end{array}$ & $\begin{array}{l}1 \times 10^{7} \\
8 \times 10^{6}\end{array}$ & $\begin{array}{l}0.62 / 0.62 \\
0.59 / 0.59\end{array}$ & $\begin{array}{l}38.1 \\
18.4\end{array}$ & $\begin{array}{l}0.27 \\
0.13\end{array}$ & $\begin{array}{l}0.03 \\
0.06\end{array}$ & $\begin{array}{l}38 \\
13 \\
\end{array}$ & $\begin{array}{r}39 / 46 \\
7 / 46\end{array}$ \\
\hline $\mathrm{p}-\mathrm{H}_{2} \mathrm{O}$ & $\begin{array}{l}1_{11}-0_{00} \\
2_{02}-1_{11}\end{array}$ & $\begin{array}{r}1113.34301 \\
987.92676\end{array}$ & $\begin{array}{r}53.4 \\
100.8\end{array}$ & $\begin{array}{l}1.84 \times 10^{-2} \\
5.84 \times 10^{-3}\end{array}$ & $\begin{array}{l}1 \times 10^{8} \\
4 \times 10^{7}\end{array}$ & $\begin{array}{l}0.63 / 0.64 \\
0.63 / 0.64\end{array}$ & $\begin{array}{l}19.0 \\
21.5\end{array}$ & & $\begin{array}{l}0.06 \\
0.07\end{array}$ & $\begin{array}{l}28 \\
36\end{array}$ & $\begin{array}{l}28 / 46 \\
25 / 46\end{array}$ \\
\hline $\mathrm{o}-\mathrm{H}_{2}^{18} \mathrm{O}$ & $1_{10}-1_{01}$ & 547.67644 & 60.5 & $3.29 \times 10^{-3}$ & $1 \times 10^{7}$ & $0.62 / 0.62$ & 38.7 & 0.27 & 0.07 & 38 & $1 / 46$ \\
\hline $\mathrm{p}-\mathrm{H}_{2}^{18} \mathrm{O}$ & $1_{11}-0_{00}$ & 1101.69826 & 52.9 & $1.79 \times 10^{-2}$ & $1 \times 10^{8}$ & $0.63 / 0.64$ & 19.0 & 0.13 & 0.06 & 28 & $0 / 46$ \\
\hline $\mathrm{C}^{18} \mathrm{O}$ & $9-8$ & 987.56038 & 237.0 & $6.38 \times 10^{-5}$ & $2 \times 10^{5}$ & $0.63 / 0.64$ & 21.5 & 0.15 & 0.07 & 36 & $4 / 46$ \\
\hline $\mathrm{CO}$ & $10-9$ & & 304.2 & $1.01 \times 10^{-4}$ & $3 \times 10^{5}$ & $0.59 / 0.59$ & 18.4 & 0.1 & 0.06 & 13 & $40 / 46$ \\
\hline${ }^{13} \mathrm{CO}$ & $10-9$ & 1101.34966 & 290.8 & $8.86 \times 10^{-5}$ & $3 \times 10^{5}$ & $0.63 / 0.64$ & 19.3 & 0.13 & 0.06 & 28 & $20 / 46$ \\
\hline
\end{tabular}

Notes. ${ }^{(a)}$ Taken from the JPL database (Pickett et al. 2010). ${ }^{(b)}$ Taken from Daniel et al. (2011) and Dubernet et al. (2009) for $\mathrm{H}_{2} \mathrm{O}$, the JPL database (Pickett et al. 2010) for $\mathrm{H}_{2}^{18} \mathrm{O}$ and CO isotopologues. ${ }^{(c)}$ Calculated for $T=300 \mathrm{~K}$. ${ }^{(d)}$ Taken from the latest HIFI calibration document at http:// herschel.esac.esa.int/twiki/pub/Public/HifiCalibrationWeb/HifiBeamReleaseNote_Sep2014.pdf. ${ }^{(e)}$ Calculated using Eq. (3) from Roelfsema et al. (2012). ${ }^{(f)}$ Total time including on+off source and overheads. ${ }^{(g)}$ Number of detections. Due to contamination of the reference positions, the status for observations of W40 sources 01,03 and 06 cannot be determined.

(HIFI, de Graauw et al. 2010) and Photodetector Array Camera and Spectrometer (PACS, Poglitsch et al. 2010) detectors between the 31st October 2012 and 27th March 2013. The observing modes, observational properties, data reduction and detection statistics are described for each instrument separately in Sects. 3.1 and 3.2. Complementary spectroscopic maps obtained through follow-up observations of the sample with ground-based facilities are then described in Sect. 3.3.

\subsection{HIFI}

\subsubsection{Observational details}

HIFI was a set of seven single-pixel dual-sideband heterodyne receivers that combined to cover the frequency ranges $480-1250 \mathrm{GHz}$ and $1410-1910 \mathrm{GHz}$ with a sideband ratio of approximately unity. Spectra were simultaneously observed in two polarisations, $H$ and $V$, which pointed at slightly different positions on the sky $\left(\sim 6.5^{\prime \prime}\right.$ apart at $557 \mathrm{GHz}$ decreasing to $\sim 2.8^{\prime \prime}$ at $1153 \mathrm{GHz}$ ), with two spectrometers simultaneously providing both wideband (WBS, $4 \mathrm{GHz}$ bandwidth at $1.1 \mathrm{MHz}$ resolution) and high-resolution (HRS, typically $230 \mathrm{MHz}$ bandwidth at $250 \mathrm{kHz}$ resolution) frequency coverage.

The HIFI component of the WILL Herschel observations consists of single pointed spectra at four frequency settings, principally targeting the $\mathrm{H}_{2} \mathrm{O} 1_{10}-1_{01}, 1_{11}-0_{00}$ and $2_{02}-1_{11}$ transitions at 557,1113 and $988 \mathrm{GHz}$ respectively and the ${ }^{12} \mathrm{CO}$ $J=10-9$ transition at $1152 \mathrm{GHz}$, which also includes the $\mathrm{H}_{2} \mathrm{O}$ $3_{12}-2_{21}$ transition. All observations were carried out in dualbeam-switch mode with a nod of $3^{\prime}$ using fast chopping. The specific central frequencies of the settings were chosen to maximise the number of observable $\mathrm{H}_{2} \mathrm{O}, \mathrm{CO}$ and $\mathrm{H}_{2}^{18} \mathrm{O}$ transitions, the details of which are given in Table 2 along with the corresponding instrumental properties, spectral and spatial resolution, and observing time. The main difference compared to the WISH HIFI observations of low-mass sources (see Kristensen et al. 2012; Mottram et al. 2014) was that the frequency of the WILL observations for the $\mathrm{H}_{2} \mathrm{O} 1_{10}-1_{01}$ and $1_{11}-0_{00}$ settings was set so that the corresponding $\mathrm{H}_{2}^{18} \mathrm{O}$ transition was observed simultaneously, and longer observing times were used for the $\mathrm{H}_{2} \mathrm{O} 1_{10}-1_{01}$ setting. The observation ID numbers for all WILL HIFI observations are given in Table B.1.
Initial data reduction was conducted using the Herschel Interactive Processing Environment (HIPE v. 10.0, Ott 2010). After initial spectrum formation, any instrumental standing waves were removed. Next, a low-order $(\leq 2)$ polynomial baseline was subtracted from each sub-band. The fit to the baseline was then used to calculate the continuum level, compensating for the dualsideband nature of the HIFI detectors (the initial continuum level is the combination of emission from both the upper and lower sideband, which we assume to be equal). Following this the WBS sub-bands were stitched into a continuous spectrum and all data were converted to the $T_{\mathrm{MB}}$ scale using the latest beam efficiencies (see Table 2). Finally, for ease of analysis, all data were converted to FITS format and resampled to $0.3 \mathrm{~km} \mathrm{~s}^{-1}$ spectral resolution on the same velocity grid using bespoke PYTHON routines.

Few differences have been found in line-shape or gain between the $H$ and $V$ polarisations (e.g. Kristensen et al. 2012; Y1ld1z et al. 2013; Mottram et al. 2014), so after visual inspection the two polarisations were co-added to improve signal-tonoise. The velocity calibration is better than $100 \mathrm{kHz}$, while the pointing uncertainty is better than $2^{\prime \prime}$ and the intensity calibration uncertainty is $\lesssim 10 \%$ (Mottram et al. 2014).

\subsubsection{Results}

Figures 2 and 3 present the observed HIFI ortho- $\mathrm{H}_{2} \mathrm{O} 1_{10}-1_{01}$ $(557 \mathrm{GHz})$ ground-state transition and ${ }^{12} \mathrm{CO} J=10-9$, respectively, for all WILL sources. The water spectra are complex, containing multiple components, some absorption, which is usually narrow, and emission up to $\pm \sim 100 \mathrm{~km} \mathrm{~s}^{-1}$ from the source velocity, similar to other Herschel HIFI observations of water towards Class 0/I sources (e.g. Kristensen et al. 2012). ${ }^{12} \mathrm{CO}$ $J=10-9$ typically shows two gaussian emission components with a lower total velocity extent than $\mathrm{H}_{2} \mathrm{O}$. Strong, narrow absorption in ${ }^{12} \mathrm{CO} J=10-9$ for W40 sources 01,03 and 06 (see Fig. 3) indicates that contamination in at least one of the reference positions affects these spectra and also likely affects most of the $\mathrm{H}_{2} \mathrm{O}$ transitions for these sources as well. The narrow yet bright nature of the ${ }^{12} \mathrm{CO} J=10-9$ seen in six sources (OPH 01 , W40 01 and W40 03-06, see Fig. 3), combined with the narrow and low-intensity nature of the $\mathrm{H}_{2} \mathrm{O}$ emission, suggests that they are related to photon-dominated regions (PDRs, cf. for example 
J. C. Mottram et al.: Outflows, infall and evolution of a sample of embedded low-mass protostars

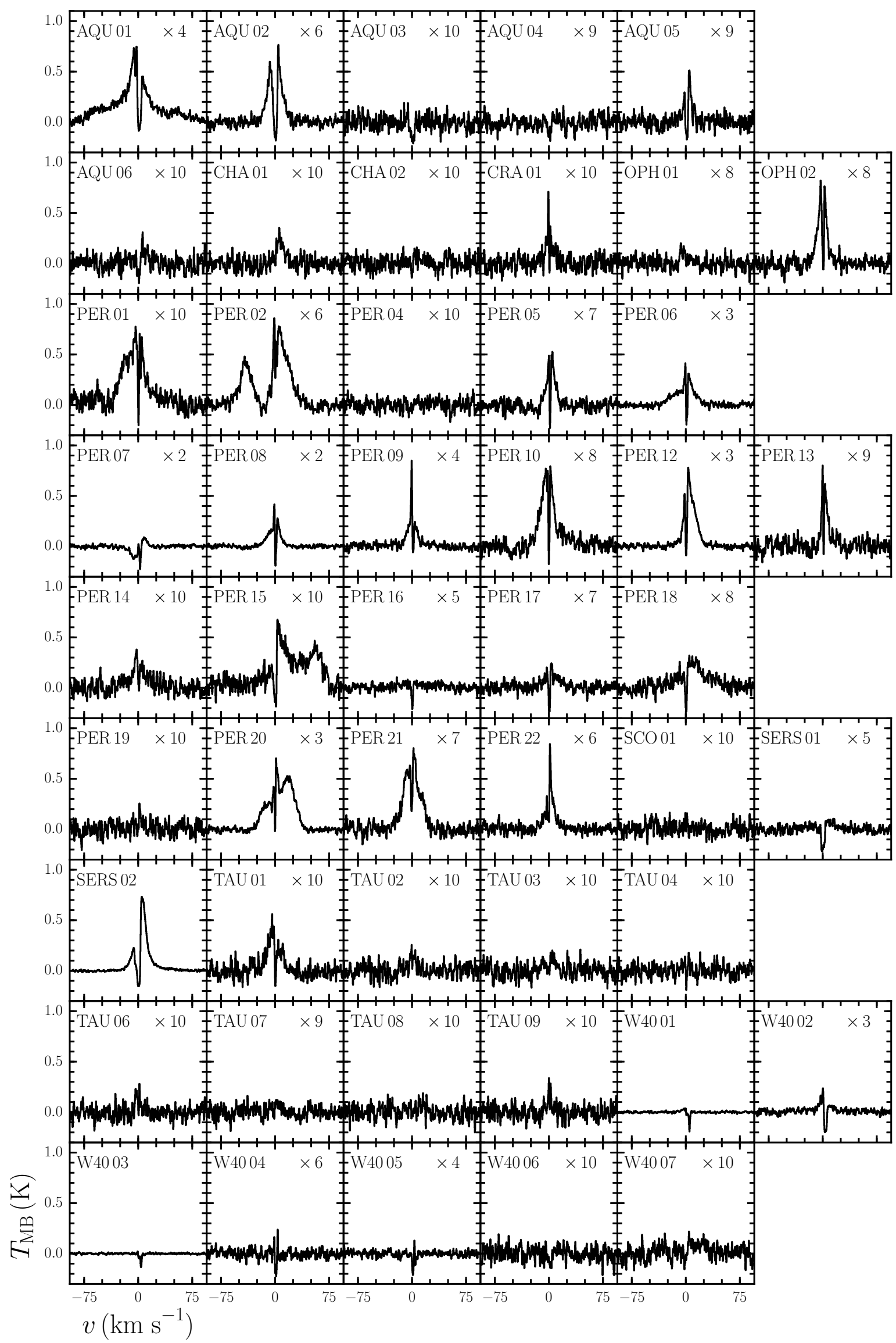

Fig. 2. $\mathrm{H}_{2} \mathrm{O} 1_{10}-1_{01}(557 \mathrm{GHz})$ continuum-subtracted spectra for the final WILL sample. All have been recentred so that the source velocity is at zero. The number in the upper-right corner of each panel indicates what factor the spectra have been multiplied by in order to show them on a common scale. 

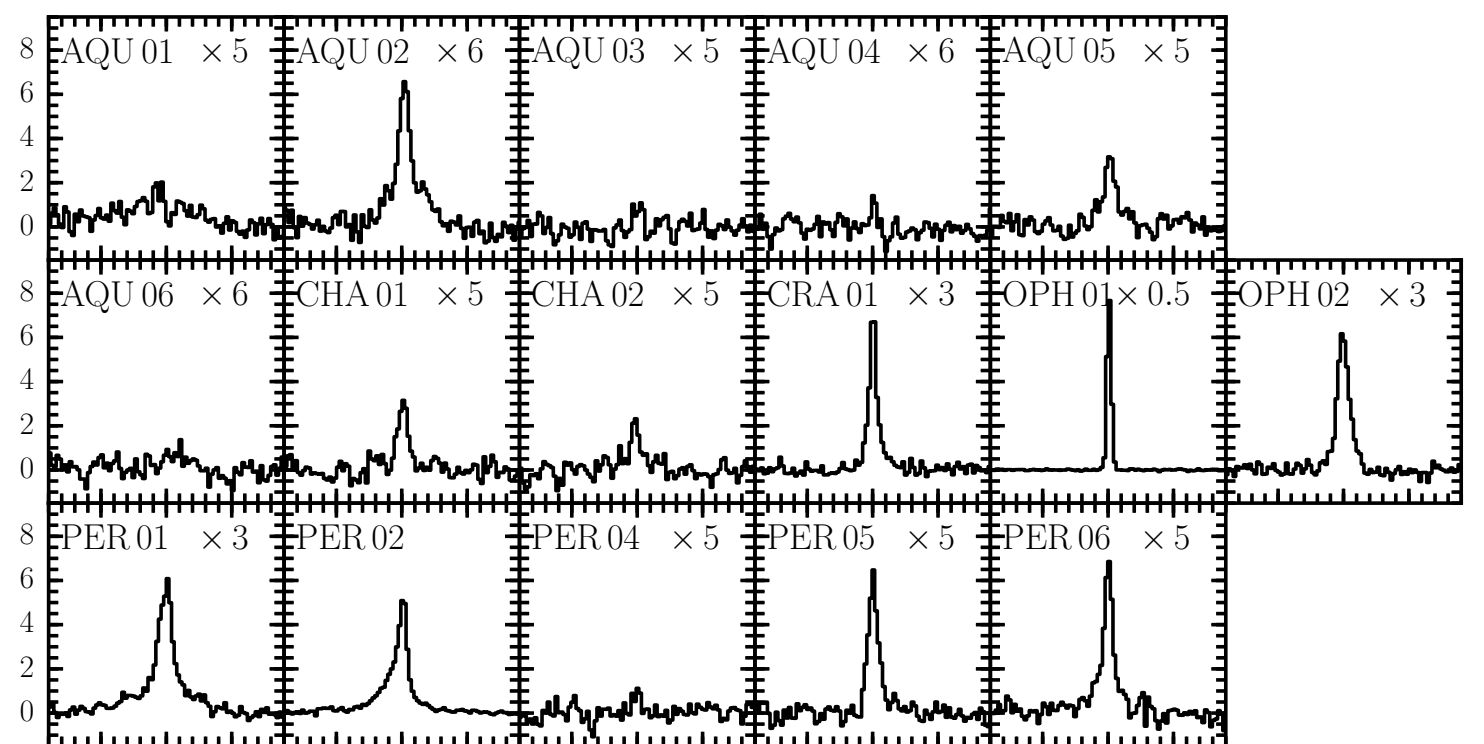

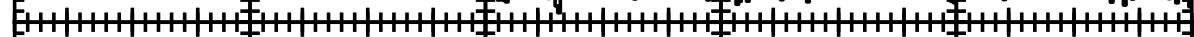
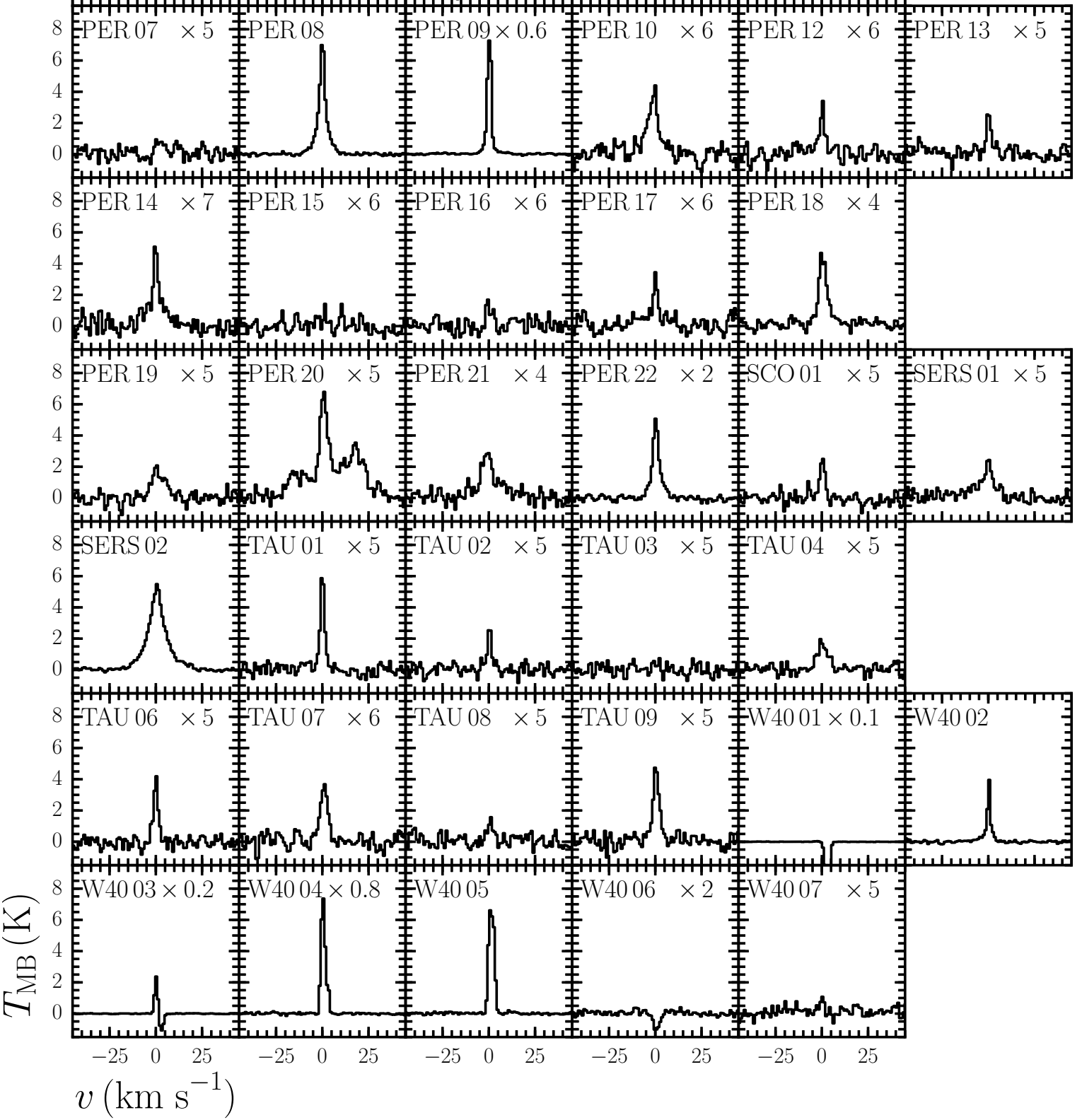

Fig. 3. CO $J=10-9$ continuum-subtracted spectra for the final WILL sample. All have been recentred so that the source velocity is at zero. The number in the upper-right corner of each panel indicates what factor the spectra have been multiplied by in order to show them on a common scale. 
Table 3. Wavelength ranges covered by WILL PACS line-scan settings.

\begin{tabular}{|c|c|c|}
\hline Setting & $\begin{array}{l}\text { Wavelengths } \\
\qquad(\mu \mathrm{m})\end{array}$ & Primary transitions \\
\hline 1 & $\begin{array}{c}78.6-79.5 \\
81.3-82.2 \\
84.2-85.0 \\
89.5-90.4 \\
123.7-126.1 \\
157.0-158.0 \\
162.5-164.5 \\
168.3-170.0 \\
179.0-180.8\end{array}$ & $\begin{array}{c}\mathrm{H}_{2} \mathrm{O} 4_{23}-3_{12}, 6_{15}-5_{24}, \mathrm{CO} 33-32, \mathrm{OH} \\
\mathrm{H}_{2} \mathrm{O} 6_{16}-5_{05}, \mathrm{CO} 32-31 \\
\mathrm{H}_{2} \mathrm{O} 7_{16}-7_{07}, \mathrm{CO} 31-30, \mathrm{OH} \\
\mathrm{H}_{2} \mathrm{O} 3_{22}-2_{11}, \mathrm{CO} 29-28 \\
\mathrm{H}_{2} \mathrm{O} 4_{04}-3_{13}, \mathrm{CO} 21-20 \\
{[\mathrm{C} \mathrm{II}]} \\
\mathrm{CO} 16-15, \mathrm{OH} \\
\mathrm{H}_{2} \mathrm{O} 2_{12}-1_{01}, 2_{21}-2_{12}\end{array}$ \\
\hline 2 & $\begin{array}{c}53.6-55.0 \\
63.0-63.5 \\
107.3-109.7 \\
189.0-190.5\end{array}$ & $\begin{array}{c}\mathrm{H}_{2} \mathrm{O} 8_{18}-7_{07},[\mathrm{OI}] \\
\mathrm{H}_{2} \mathrm{O} 2_{21}-1_{10}, \mathrm{CO} 24-23\end{array}$ \\
\hline
\end{tabular}

CO observations of the Orion Bar PDR, Hogerheijde et al. 1995; Jansen et al. 1996; Nagy et al. 2013).

The detection statistics for all transitions are given in the last column of Table 2, excluding W40 sources 01,03 and 06 due to the contamination of these spectra. The $\mathrm{H}_{2} \mathrm{O} 1_{10}-1_{01}$ transition is detected towards 39/46 sources in total, including 33/36 confirmed Class 0/I sources (not detected in CHA 02, PER 04 and W40 07, see Fig. 2), while ${ }^{12} \mathrm{CO} J=10-9$ is detected towards 40/46 sources in total including 32/36 Class 0/Is (not detected in CHA 02, PER 07, PER 15 and W40 07). $\mathrm{H}_{2}^{18} \mathrm{O} 1_{10}-1_{01}$ is only detected towards the source with the strongest $\mathrm{H}_{2} \mathrm{O}$ emission, SERS 02, while $\mathrm{C}^{18} \mathrm{O} J=9-8$ is only detected towards four sources (PER 02, SERS 02, W40 04 and 05).

A more detailed analysis of the kinematics of the HIFI lines is presented and discussed in San José-García (2015), including the results of Gaussian decomposition of the lines using the methods outlined for the WISH sample by Mottram et al. (2014) and San José-García et al. (2013) for $\mathrm{H}_{2} \mathrm{O}$ and $\mathrm{CO}$, respectively. In summary, the minimum number of Gaussian components is found that results in no residuals above $3 \sigma$, with these components then categorised between the envelope and $\mathrm{C}$ or $J$-type outflow-related shocks depending on their width and offset from the source velocity. A global fit is used for the $\mathrm{H}_{2} \mathrm{O}$ transitions with the component peak velocity and line-widths constrained by all lines and the intensity allowed to vary between transitions because the lines all have a consistent shape. The different $\mathrm{CO}$ transitions are fit independently as their line profile shapes vary between different transitions.

\subsection{PACS}

\subsubsection{Observational details}

PACS consisted of four detectors, two photoconductor arrays with $16 \times 25$ pixels for integral field unit (IFU) spectroscopy and two bolometer arrays with $16 \times 32$ and $32 \times 64$ pixels for broad-band imaging photometry. In IFU spectroscopy mode, observations were taken simultaneously in the red 1st order grating $(102-210 \mu \mathrm{m})$ and one of the $2 \mathrm{nd}$ or $3 \mathrm{rd}$ order blue gratings (51-73 $\mu \mathrm{m}$ or $71-105 \mu \mathrm{m})$ over $5 \times 5$ spatial pixels (spaxels), which covered a $47^{\prime \prime} \times 47^{\prime \prime}$ field of view. For details of the 70, 100 and $160 \mu \mathrm{m}$ PACS (and 250, 350 and $500 \mu \mathrm{m}$ SPIRE, Griffin et al. 2010) photometric maps used to determine the continuum flux densities for the SEDs (discussed in Sect. A.1) see André et al. (2010).
WILL PACS observations were carried out using the IFU in line-scan mode where deep observations were obtained for targeted wavelength regions (bandwidth $\Delta \lambda / \lambda=0.01$ ) around selected transitions. Two wavelength settings were used, each including observations in both the blue and red gratings, as summarised in Table 3. The principle transitions within these regions are from $\mathrm{H}_{2} \mathrm{O}, \mathrm{OH},[\mathrm{OI}$, $\mathrm{CO}$ and [C II], the properties of which are given in Table A.3. While WILL targeted the key lines observed by WISH, some of the wavelength ranges were shifted slightly in order to allow for better baseline subtraction or additional line detections (e.g. those around 82 and $90 \mu \mathrm{m}$ were shifted to slightly longer wavelengths) while others were omitted to save time (e.g. around CO $J=14-13$ ). The velocity resolution of PACS ranges from $75 \mathrm{~km} \mathrm{~s}^{-1}$ at the shortest wavelength to $300 \mathrm{~km} \mathrm{~s}^{-1}$, with only [O I] sometimes showing velocity resolved line profiles in a few sources. All observations used a chopping/nodding observing mode with off-positions within $6^{\prime}$ of the target coordinates. The obsids for WILL PACS observations are given in Table B.1. For one source, TAU 08, PACS data were not obtained because the coolant on Herschel ran out before they could be successfully observed.

Data reduction was performed with HIPE v.10 with Calibration Tree 45, including spectral flat-fielding (see Herczeg et al. 2012; Green et al. 2013a, for more details). The flux density was normalised to the telescopic background and calibrated using observations of Neptune, resulting in an overall calibration uncertainty in flux densities of approximately $20 \%$ (Karska et al. 2014b). 1D spectra were obtained by summing over a number of spaxels chosen after inspection of the 2D spectral maps (Karska et al. 2013), with only the central spaxel used for pointlike emission multiplied by the wavelength-dependent instrumental correction factors to account for the PSF (see PACS Observers $\mathrm{Manual}^{2}$ ).

\subsubsection{Results}

An overview of the PACS spectra for all sources is shown in Fig. 4, while an overview of the detection of all transitions is given in Table A.4. An extensive analysis of the PACS data for WILL sources in the Perseus molecular cloud was published in Karska et al. (2014b), while a global study of PACS spectroscopy towards all WILL, DIGIT and WISH sources will be presented in Karska et al. (in prep.). Line flux densities were extracted from the PACS data as described in Karska et al. (2013).

The detection statistics for the main transitions are also given in Table A.4. The most frequently detected line is [O I], which is detected in 42 out of 48 sources. Those sources not showing [O I] detections (AQU 03-06, PER 13 and W40 06) are generally not detected in other PACS lines. These sources have weak and/or narrow lines, where detected, in the HIFI observations (cf. Fig. 2). There are 30 sources detected in at least one PACS water transition, while 27 are detected in at least one $\mathrm{OH}$ line and 32 in at least one $\mathrm{CO}$ line, with a detection more likely in the lower-energy transitions.

\subsection{Ground-based follow-up}

Follow-up ground-based observations were conducted towards the WILL sample, where not already available, to complement the Herschel spectral line information. Approximately half of

2 http://herschel.esac.esa.int/Docs/PACS/html/pacs_om. html 


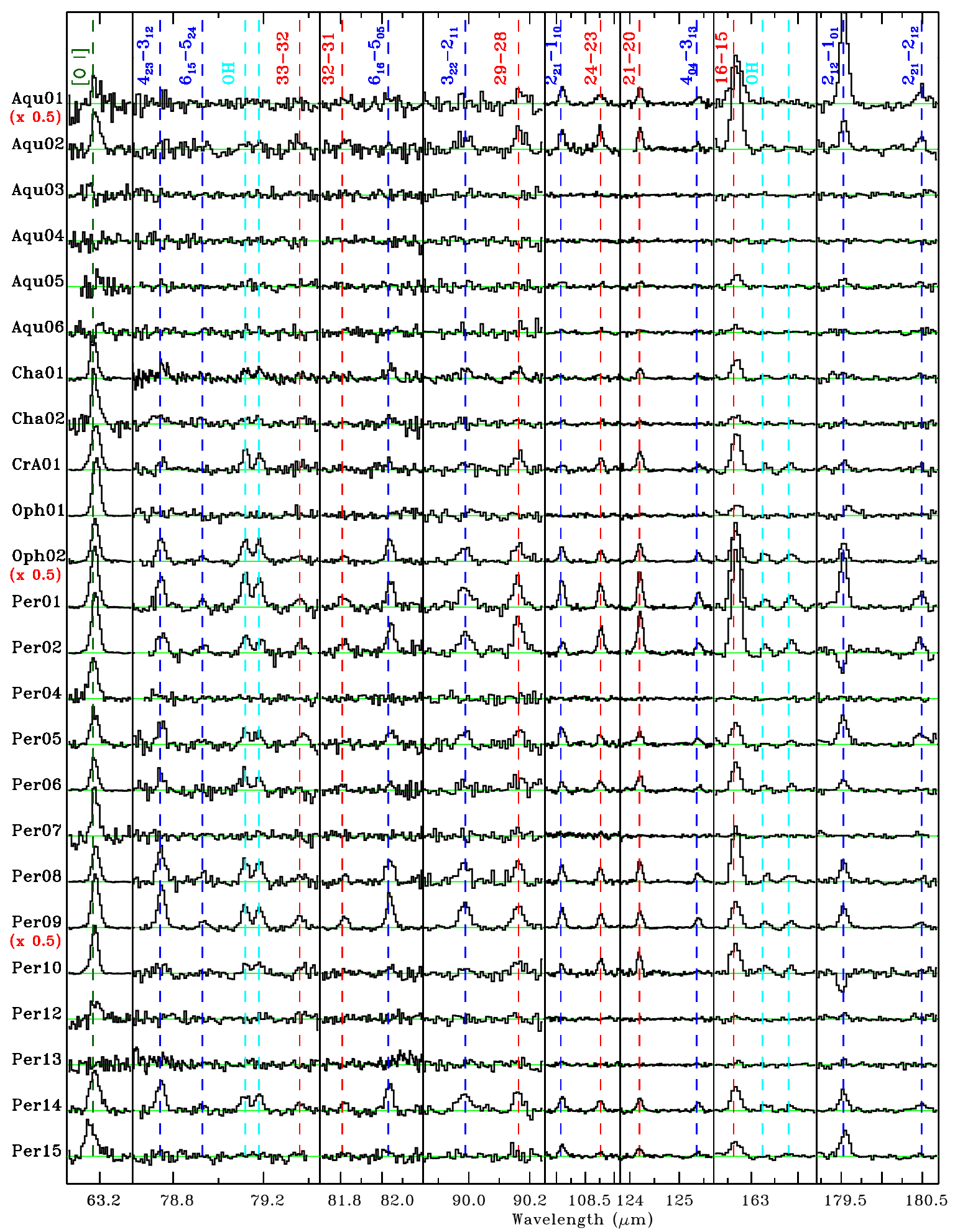

Fig. 4. Overview of continuum-subtracted PACS spectra for selected lines. These are not corrected for the PSF. $\mathrm{H}_{2} \mathrm{O}, \mathrm{CO}$ and $\mathrm{OH}$ lines are marked in blue, red and cyan, respectively, with the [O I] marked in green. The $y$-axis of each spectrum for all lines except [O I] goes from 0 to 5 Jy, with the brightest sources scaled down by the factor indicated in red below the source name. The [O I] spectra are scaled separately by a factor between 0.05 and 1 .

the sources in the final catalogue were not part of the samples and regions already observed in $\mathrm{HCO}^{+} J=4-3$ by Carney et al. (2016), so such observations were undertaken to confirm the embedded protostellar nature of the sample (see Appendix A.7).
The follow-up observations also included maps of ${ }^{12} \mathrm{CO} \mathrm{J}=$ $3-2$ to characterise the entrained molecular outflow and $\mathrm{C}^{18} \mathrm{O}$ $J=3-2$ to obtain the source velocity and turbulent line-width in the cold envelope. 
J. C. Mottram et al.: Outflows, infall and evolution of a sample of embedded low-mass protostars

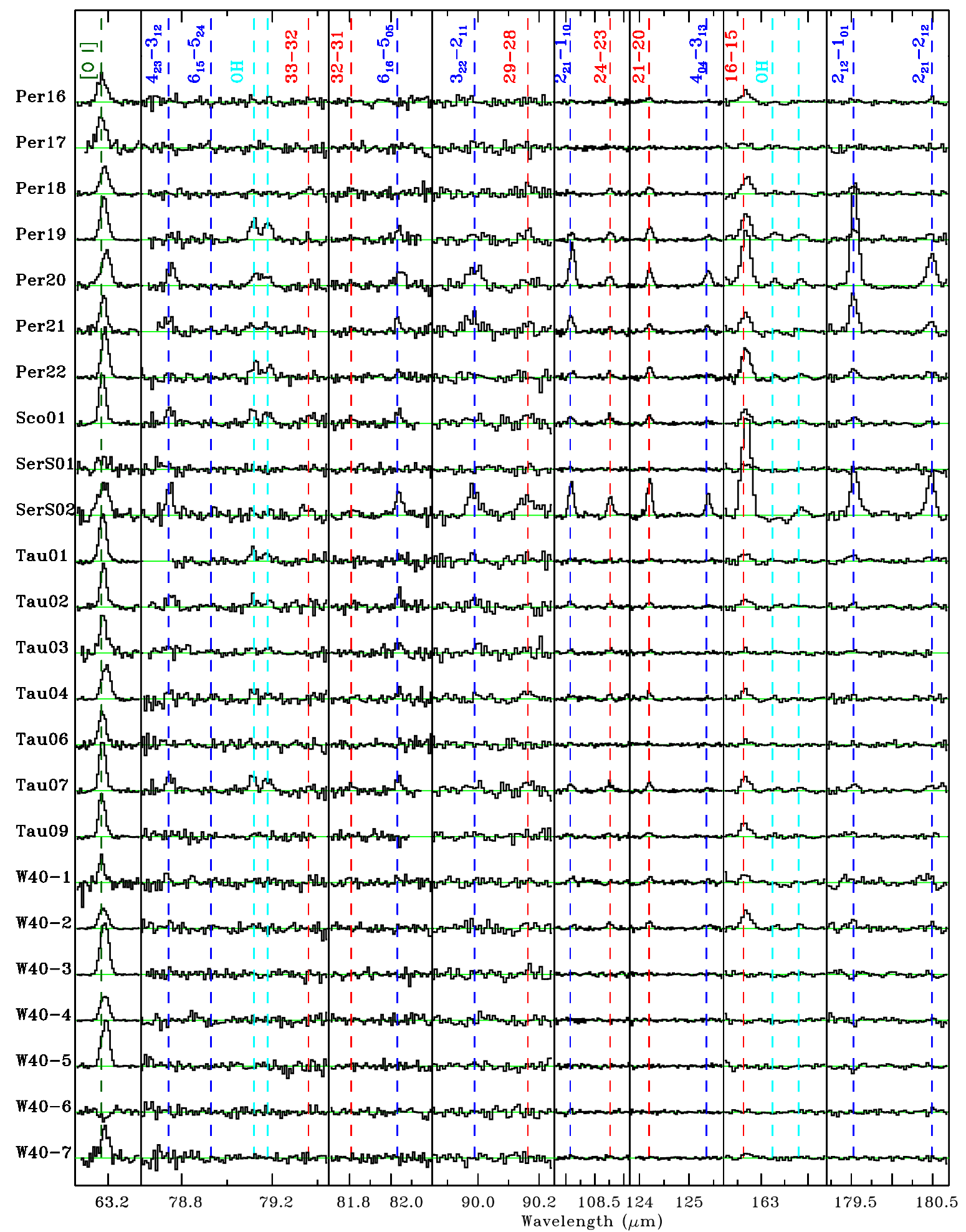

Fig. 4. continued.

All but the two WILL sources in Chameleon are observable from the James Clerk Maxwell Telescope $\left(\mathrm{JCMT}^{3}\right)$ on Mauna Kea, Hawaii. Observations of $\mathrm{C}^{18} \mathrm{O} J=3-2$ and $\mathrm{HCO}^{+} J=4-3$ were obtained using HARP (Buckle et al. 2009) and the ACSIS

3 The James Clerk Maxwell Telescope has historically been operated by the Joint Astronomy Centre on behalf of the Science and Technology Facilities Council of the United Kingdom, the National Research Council of Canada and the Netherlands Organisation for Scientific Research autocorrelator at the JCMT as $2^{\prime} \times 2^{\prime}$ jiggle maps either as part of observing programs M12AN08 and M12BN07 or from the archive where these were already taken as part of other programs. These also included $2^{\prime} \times 2^{\prime}$ jiggle map observations of all sources in ${ }^{12} \mathrm{CO}$ and $\mathrm{H}^{13} \mathrm{CO}^{+} J=4-3$, while ${ }^{13} \mathrm{CO} J=3-2$ was obtained simultaneously with $\mathrm{C}^{18} \mathrm{O} J=3-2$ for those sources that had not been previously observed. In a few cases, the ${ }^{12} \mathrm{CO}$ and ${ }^{13} \mathrm{CO}$ observations were supplemented with cut-outs from 
the large basket-woven raster maps taken as part of the JCMT Gould Belt survey observations of Perseus, Taurus and Ophiuchus (Curtis et al. 2010; Davis et al. 2010; White et al. 2015).

For the two Chameleon sources, a series of lines were observed with the Atacama Pathfinder EXperiment (APEX ${ }^{4}$ ) telescope at Llano de Chajnantor, Chile as part of project M0002_90. These consisted of $2^{\prime} \times 2^{\prime}$ on-the-fly maps of ${ }^{12} \mathrm{CO}$ $J=3-2$ and $2-1$, as well as single pointings of ${ }^{12} \mathrm{CO} J=4-3$, ${ }^{13} \mathrm{CO}, \mathrm{C}^{18} \mathrm{O}$ and $\mathrm{C}^{17} \mathrm{O} J=3-2$ and $2-1$, and $\mathrm{HCO}^{+}$and $\mathrm{H}^{13} \mathrm{CO}^{+}$ $J=4-3$, using the $\mathrm{FLASH}^{+}$(for the $300 \mathrm{GHz}$ and $450 \mathrm{GHz}$ bands) and APEX1 (for the $225 \mathrm{GHz}$ band, Vassilev et al. 2008) receivers.

The initial reduction of the JCMT jiggle maps was performed using the most up-to-date version of the STARLINK ${ }^{5}$ reduction package ORAC-DR (Jenness et al. 2015). Similar initial reduction was performed for the APEX data using GILDAS-CLASS ${ }^{6}$. Following this, all data were (re-)baselined, corrected to the $T_{\mathrm{mb}}$ scale, and re-sampled to a common velocity scale with $0.2 \mathrm{~km} \mathrm{~s}^{-1}$ resolution using customised PYTHON scripts. A summary of the observed lines, adopted beam efficiencies and typical $\sigma_{\text {rms }}$ values obtained is presented in Table A.8. ${ }^{12} \mathrm{CO}$ emission is detected towards all sources but not all show evidence of outflows (see Sects. 4.1 and A.4 for more details). More details of detections and non-detections in the ${ }^{13} \mathrm{CO}, \mathrm{C}^{18} \mathrm{O}, \mathrm{C}^{17} \mathrm{O}, \mathrm{HCO}^{+}$ and $\mathrm{H}^{13} \mathrm{CO}^{+}$spectra can be found in Sect. A.6.

\section{Outflow characteristics and energetics}

In this section we present selected characterisation and comparative analysis of the Herschel and ground-based spectral line observations, focusing on the entrained outflow as probed by ${ }^{12} \mathrm{CO}$ $J=3-2$ and outflow/wind/jet-related shocks traced by PACS [OI] observations and the broader components of the HIFI $\mathrm{H}_{2} \mathrm{O}$ and ${ }^{12} \mathrm{CO} J=10-9$ lines. In this and the following section, the pre-stellar and Class II sources are excluded from all analyses as they do not show strong outflow or envelope signatures (see Sect. A.7 for characterisation of sources).

Details of how the various entrained outflow-related properties (i.e. mass, momentum, energy, force and mass-loss rate, maximum velocity, dynamical time, inclination and radius) were measured are given in Appendix A.4, along with a table of their values for all WILL sources with detected outflows (Table A.6). For consistency, the calculations are performed following the same method as that used by Yildiz et al. (2015) for the WISH sources, thus ensuring consistency between the WISH and WILL measurements.

\subsection{Low-J CO emission}

One simple, initial question to ask is whether or not the observations are consistent with the common assumption that all embedded protostars have outflows. Overall 34/37 (92\%) of the Class 0/I sources in the WILL sample show outflow emission associated with the source in $\mathrm{CO} J=3-2$ (shown in Fig. A.2), Two of the Class II sources (CHA 01 and TAU 03) also show outflow activity, which is discussed further in Appendix C. Of the three Class 0/I sources without detections, Per 12, a Class 0 source, shows indications in Spitzer images that the outflow is

\footnotetext{
4 APEX is a collaboration between the Max-Planck-Institut für Radioastronomie, the European Southern Observatory, and the Onsala Space Observatory.

5 http://starlink. eao.hawaii.edu/starlink

6 http://www. iram. fr/IRAMFR/GILDAS
}

in the plane of the sky (see Fig. 19 in Tobin et al. 2015, and associated discussion). Cha 02, a Class I, is faint or not detected in most tracers, but is detected in [O I] with PACS. W4001, a Class 0, is also not detected in most PACS lines but shows a faint broad blue-shifted line-wing in the HIFI spectra. Thus the lack of detection for these three sources is likely due to sensitivity, meaning our observations are consistent with the hypothesis that all Class 0/I sources drive a molecular outflow.

An important next step in understanding the mechanism and impact of outflows on star formation is to constrain how the properties of outflows are related to those of the protostar. Figure 5 shows comparisons of the full-width at zero intensity (FWZI), which is the sum of the maximum velocities in the red and blue outflow lobes, mass in the entrained outflow $\left(M_{\text {out }}\right)$, the mass entrainment rate in the outflow $\left(\dot{M}_{\text {out }}\right)$ and the timeaveraged momentum or outflow force $\left(F_{\mathrm{CO}}\right)$ measured from $\mathrm{CO}$ $J=3-2$ with $L_{\mathrm{bol}}, T_{\mathrm{bol}}$ and $M_{\mathrm{env}}$ for all WISH and WILL Class $0 /$ I sources. $\dot{M}_{\text {out }}$ and $F_{\mathrm{CO}}$, as calculated quantities, are corrected for the inclination (see Appendix A.4 for details), while we do not correct for inclination for directly measured quantities, such as FWZI and $M_{\text {out }}$.

The strongest correlation $(5.9 \sigma)$ is between the outflow mass and envelope mass, perhaps unsurprisingly given that the outflow is entrained from the envelope, with $M_{\text {out }} / M_{\text {env }}$ centred around $1 \%$, as shown by the grey dashed line. A significant, though weaker, correlation is also found between outflow mass and luminosity $(3.4 \sigma)$, possibly due to the correlation between $L_{\mathrm{bol}}$ and $M_{\text {env }}$ (see Fig. 1). Since the mass is also an important factor in the calculation of $\dot{M}_{\text {out }}$ and $F_{\mathrm{CO}}$, it is not surprising that both also show significant (i.e. $\geq 3 \sigma$ ) correlations with $M_{\text {env }}$ and $L_{\text {bol }}$. In general, all parameters decrease between Class 0 and Class I, as reflected in the significant negative correlations with $T_{\text {bol }}$ seen for FWZI, $M_{\text {out }}, \dot{M}_{\text {out }}$ and $F_{\mathrm{CO}}(3.9,3.8,3.6$ and $3.1 \sigma$ respectively).

Correlations of $F_{\mathrm{CO}}$ with $L_{\mathrm{bol}}$ and $M_{\mathrm{env}}$ have been known for some time, including Cabrit \& Bertout (1992) who found the relationship between $F_{\mathrm{CO}}$ and $L_{\text {bol }}$ indicated by the dotdashed line in Fig. 5 for a sample of Class 0 sources, and Bontemps et al. (1996) who found the relationships indicated by the solid lines for a sample of primarily Class I sources. These have subsequently been confirmed to hold when extended to the high-mass regime for a sample of young protostars in Cygnus-X by Duarte-Cabral et al. (2013) and for a sample of massive young stellar objects (MYSOs) and young H II regions by Maud et al. (2015). The relationships seen between these variables in the combined WISH and WILL sample are steeper than that found by Cabrit \& Bertout (1992) and Bontemps et al. (1996). This may be due to differences in the calculation method (van der Marel et al. 2013), or to the fact that the luminosities were likely overestimated and the $F_{\mathrm{CO}}$ values underestimated in these previous studies due to the larger beam and lower sensitivity of older observations.

At first glance, the lower right panel of Fig. 5 would seem to show a slight offset in the $F_{\mathrm{CO}}$ measurements between the WISH and WILL samples, suggesting that either there is a difference in the measurements or that they come from distinct populations. However, there is no distinct break between the WISH and WILL sources, or between Class 0 and I when considering $M_{\text {env }}$ vs. $M_{\text {out }}$ and $\dot{M}_{\text {out }}$, so the WISH sources are merely the extreme upper end of a continuous distribution. The WISH Class 0 sources were all chosen to be strong outflow sources, and the $M_{\text {out }}$ and $\dot{M}_{\text {out }}$ panels of Fig. 5 suggest that they are more prominent due to a larger reservoir of material (i.e. larger $M_{\text {env }}$ ), rather 
J. C. Mottram et al.: Outflows, infall and evolution of a sample of embedded low-mass protostars

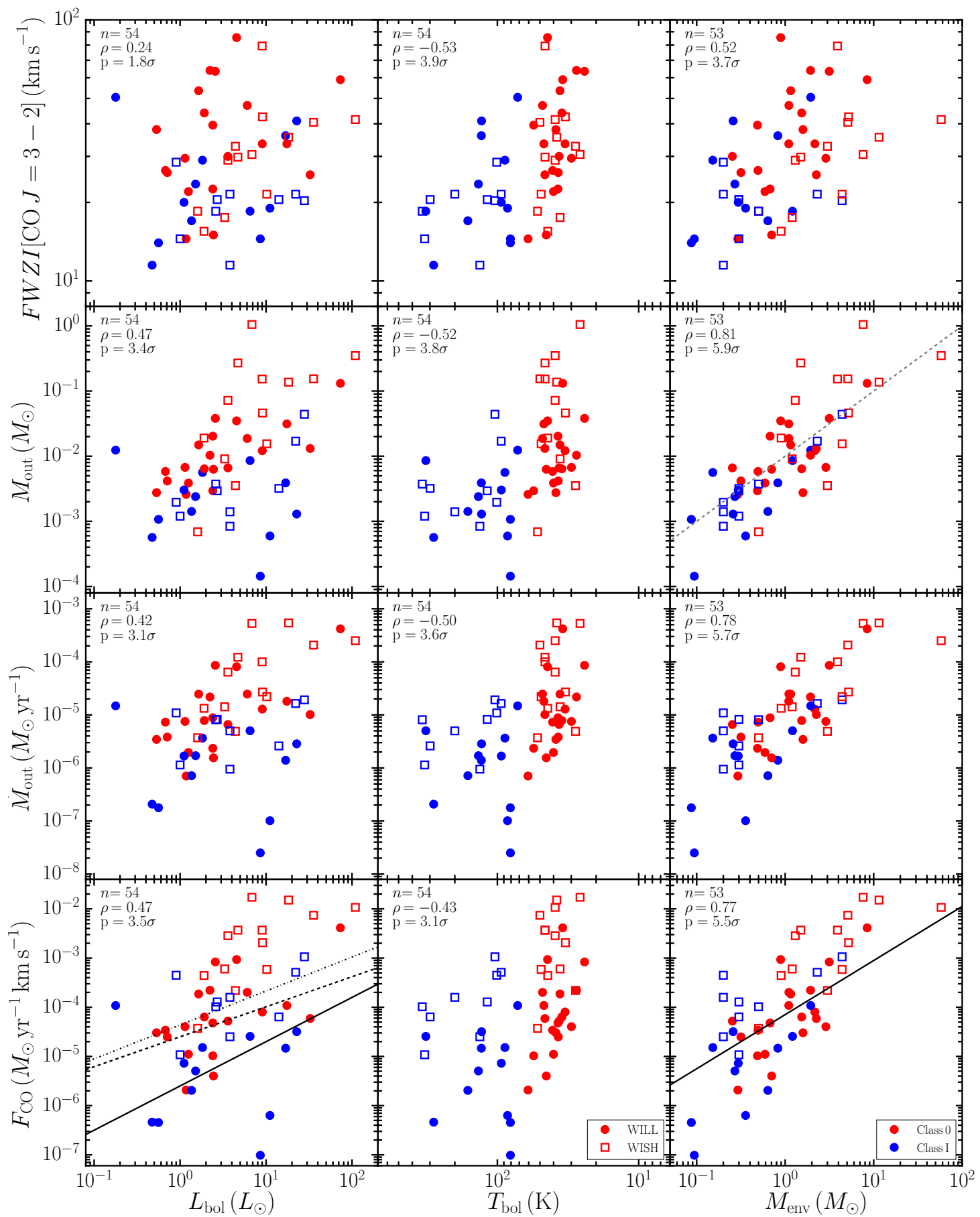

Fig. 5. Comparison of FWZI, $M_{\text {out }}, \dot{M}_{\text {out }}$ and $F_{\text {CO }}$ obtained from CO $J=3-2$ maps with $L_{\text {bol }}, T_{\text {bol }}$ and $M_{\text {env }}$ for the WILL (filled symbols) and WISH (open symbols) sources. The number of sources, correlation coefficient and probability that the correlation is not simply due to random distributions in the variables are shown in the upper-left of each panel. The grey dashed line in the panel for $M_{\text {out }}$ vs. $M_{\text {env }}$ indicates where $M_{\text {out }} / M_{\text {env }}=1 \%$. The solid black lines show the relations found by Bontemps et al. (1996) between $F_{\mathrm{CO}}, L_{\mathrm{bol}}$ and $M_{\text {env }}$ for a sample of Class I sources. The dot-dashed black line shows the best-fit found between $F_{\mathrm{CO}}$ and $L_{\mathrm{bol}}$ by Cabrit \& Bertout (1992) for a sample of Class 0 sources, while the dashed black line shows an extension to the low-mass regime of the fit to a sample of massive young sources from Maud et al. (2015).

than faster outflows as they have similar or even lower FWZI than the WILL sources.

For the Class I sources, there is little difference between the WISH and WILL sources in FWZI or $M_{\text {out }}$, but the WISH Class I sources tend to have smaller outflows (see Table A.6 for WILL sources and Table 3 in Yildiz et al. 2015, for WISH sources) resulting in larger values for the WISH sources of $\dot{M}_{\text {out }}$ and $F_{\text {CO. }}$. This could be because the WISH Class I sources are typically in smaller, more isolated clouds with shorter distances from the protostar to the cloud edge than the Class I sources in WILL.

Let us now consider the physical implications of the main correlations between outflow and source properties. The correlation between $F_{\mathrm{CO}}$ and (current) $M_{\mathrm{env}}$ is often interpreted as the result of an underlying link between envelope mass and the mass accretion rate $\left(\dot{M}_{\text {acc }}\right)$, which is itself related to the driving of the outflow (Bontemps et al. 1996; Duarte-Cabral et al. 2013). 

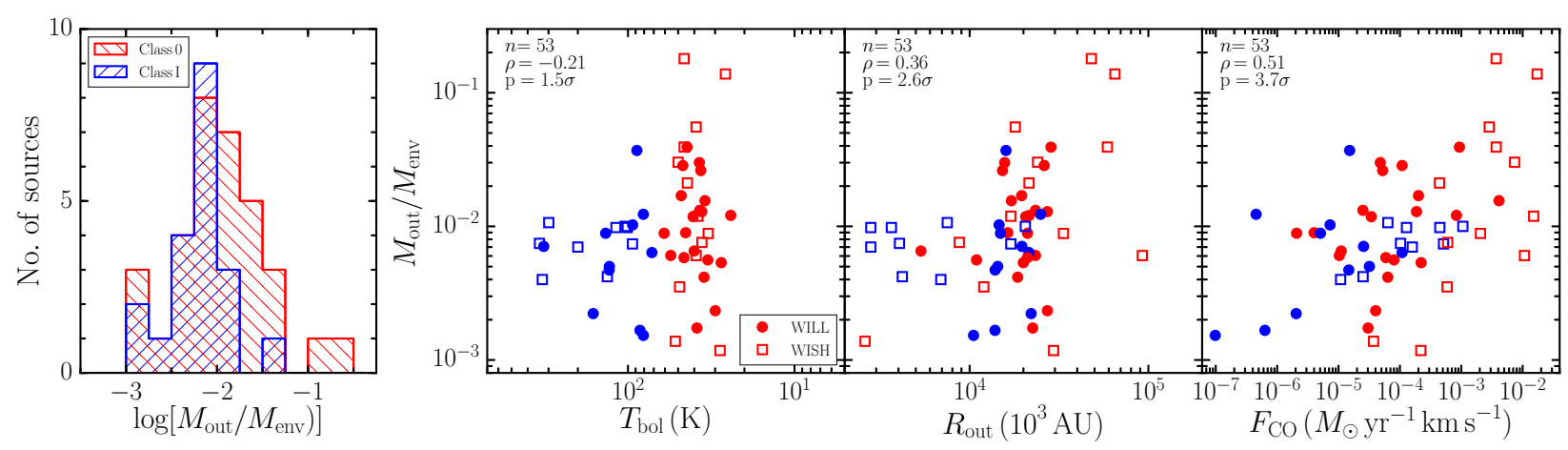

Fig. 6. Histogram of the ratio of outflow to envelope mass (i.e. $M_{\text {out }} / M_{\text {env }}$ ) for Class 0 (red) and I (blue) sources (left), as well as how this varies with $T_{\text {bol }}$ (middle-left), the mean length of the outflow lobes $\left(R_{\text {out }}\right.$, middle-right $)$ and outflow force $\left(F_{\mathrm{CO}}, r i g h t\right)$. The colours and symbols have the same meaning as in Fig. 5.

As the central source evolves, $M_{\text {env }}$ and $\dot{M}_{\text {acc }}$ decrease, leading naturally to the decrease in $F_{\mathrm{CO}}$ and other outflow-related properties between Class 0 and I sources. The comparatively tight relationship between $M_{\text {out }}$ and $M_{\text {env }}$ further supports this interpretation.

Indeed, the relation between $M_{\text {out }}$ and $M_{\text {env }}$ requires more investigation in its own right. Figure 6 shows a histogram of the fraction of mass in the outflow compared to the envelope (i.e. $M_{\text {out }} / M_{\text {env }}$ ), as well as how this varies with $T_{\text {bol }}$ (as a more continuous proxy for source evolution), the mean length of the outflow lobes $\left(R_{\text {out }}\right)$, and the strength of the outflow as measured by $F_{\mathrm{CO}}$. The values of $M_{\text {out }} / M_{\text {env }}$ vary between $\sim 0.1$ and $10 \%$, peaking around $1 \%$. The peak is similar between Class 0 and I with no significant trend with $T_{\text {bol }}$, except that the Class 0 sources extend to larger values. This seems to be related to some Class 0 sources having longer outflows (i.e. larger $R_{\text {out }}$ ) and thus have likely entrained additional material from the clump/cloud outside their original envelope. A statistically significant $(3.7 \sigma)$ correlation with $F_{\mathrm{CO}}$ is found, though with more than an order of magnitude spread.

The first impression of the peak value of $M_{\text {out }} / M_{\text {env }}$ being $\sim 10^{-2}$ in the histogram shown in Fig. 6 might be that this is rather low compared to a "typical" star formation efficiency of 30-50\% (e.g. Myers 2008; Offner et al. 2014; Frank et al. 2014, and references therein). In order to understand whether or not this value is actually reasonable, let us first assume that the outflow is responsible for removing all of the envelope material that does not end up on the star. In this case, the average mass entrainment rate in the outflow over the Class $0+\mathrm{I}$ lifetime $\left(\tau_{\text {Class } 0 / \mathrm{I}}\right)$ is given by:

$\dot{M}_{\text {out }}=\left(1-\epsilon_{\text {sf }}\right) \frac{M_{\text {env }}}{\tau_{\text {Class } 0+\mathrm{I}}}$,

where $\epsilon_{\mathrm{sf}}$ is the core-to-star formation efficiency, that is, the fraction of the envelope that will end up on the star. The observed mass-loss rate in the outflow, averaged along the flow, is given by:

$M_{\text {out }}=\dot{M}_{\text {out }} t_{\text {dyn }}$,

where $t_{\text {dyn }}$ is the dynamical time of the flow.

It is worth pointing out that $t_{\text {dyn }}$ is not necessarily the age of the source, particularly if outflow activity is time-variable. Indeed, if ejection stops then, after some time, radiative losses and mixing with the ambient cloud material will dissipate all the angular momentum and energy from the flow, meaning that the observed $t_{\mathrm{dyn}}$ is likely a lower limit to the true "age" of total accretion/outflow activity in a given source. However, if we assume that the overall mass outflow rate for a given burst is not significantly different from the average over the lifetime of the main accretion (i.e. Class $0+\mathrm{I}$ ) phase, or equally that protostellar outflows have an approximately constant entrainment efficiency per unit length, then we can combine and re-arrange Eqs. (3) and (4) to get:

$\frac{M_{\text {out }}}{M_{\text {env }}}=\left(1-\epsilon_{\text {sf }}\right) \frac{t_{\text {dyn }}}{\tau_{\text {Class } 0+\mathrm{I}}}$.

The ratio of $t_{\text {dyn }}$ to $\tau_{\text {Class } 0+\mathrm{I}}$ effectively expresses the duty cycle of the outflow.

For $\tau_{\text {Class } 0 / \mathrm{I}} \approx 0.5 \mathrm{Myr}$ (Dunham et al. 2015; Heiderman \& Evans 2015; Carney et al. 2016) and a typical dynamical time for the outflow of approximately $10^{4} \mathrm{yr}$, the ratio of outflow to envelope mass has a value of $\sim 0.01$ if the $\epsilon_{\mathrm{sf}}=0.5$. Thus, while certainly missing some details, and being affected by variation from source to source and with time, the fact that we find median value for $M_{\text {out }} / M_{\text {env }}$ of approximately $1 \%$ is consistent with protostellar outflows having an approximately constant entrainment efficiency per unit length, a core-to-star formation efficiency of approximately $50 \%$, and an outflow duty cycle of order $\sim 5 \%$.

\subsection{HIFI water and mid-J CO emission}

The water and CO $J=10-9$ spectra of many of the WILL sources show broad line wings, indicative of outflow emission, as seen in previous WISH observations (see e.g. Fig. 2 and Kristensen et al. 2012; San José-García et al. 2013, 2016; Mottram et al. 2014). The FWZI of $\mathrm{H}_{2} \mathrm{O}$ is strongly correlated with CO $J=10-9$ and $J=3-2$ (see Fig. 7), with $\mathrm{H}_{2} \mathrm{O}$ consistently tracing faster material than these $\mathrm{CO}$ transitions, typically by a factor of $\sim 2$. The narrower line-widths of CO $J=10-9$ with respect to CO $J=3-2$ are likely because the CO $J=3-2$ FWZI is calculated as the difference of the maximum red and blue velocity offsets anywhere in the $2^{\prime} \times 2^{\prime}$ maps while for $\mathrm{CO}$ $J=10-9$ this is measured from a single HIFI spectrum with a $18.4^{\prime \prime}$ beam centred at the source position, that is, over a smaller region.

Figure 8 shows a comparison of various source and outflowrelated properties with the integrated intensity of the $\mathrm{H}_{2} \mathrm{O}$ $1_{10}-1_{01}(557 \mathrm{GHz})$ line after scaling to a common distance of 200 pc. A linear scaling is used because the emission is dominated by outflows, which likely fill the beam along the outflow axis but not perpendicular to it (see Mottram et al. 2014, for more details). We are able to confirm the strong correlation 

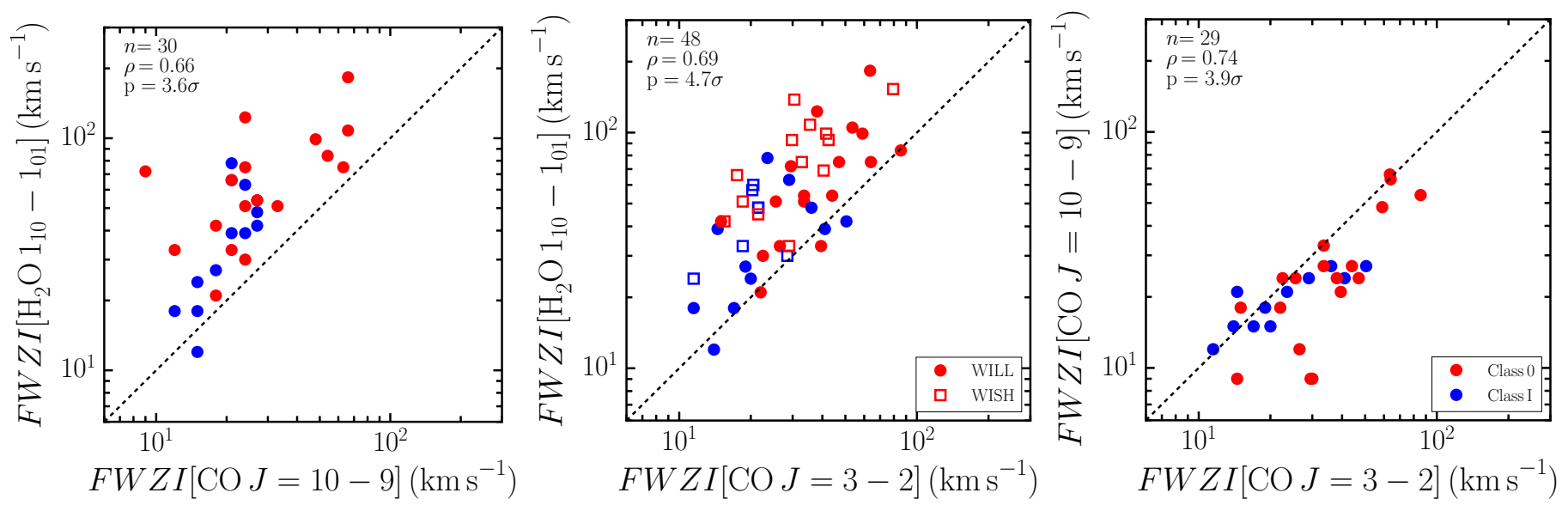

Fig. 7. Comparison of the full-width at zero intensity widths of $\mathrm{H}_{2} \mathrm{O} 1_{10}-1_{01}, \mathrm{CO} J=10-9$ and 3-2. The dashed black lines indicate the line of equality.
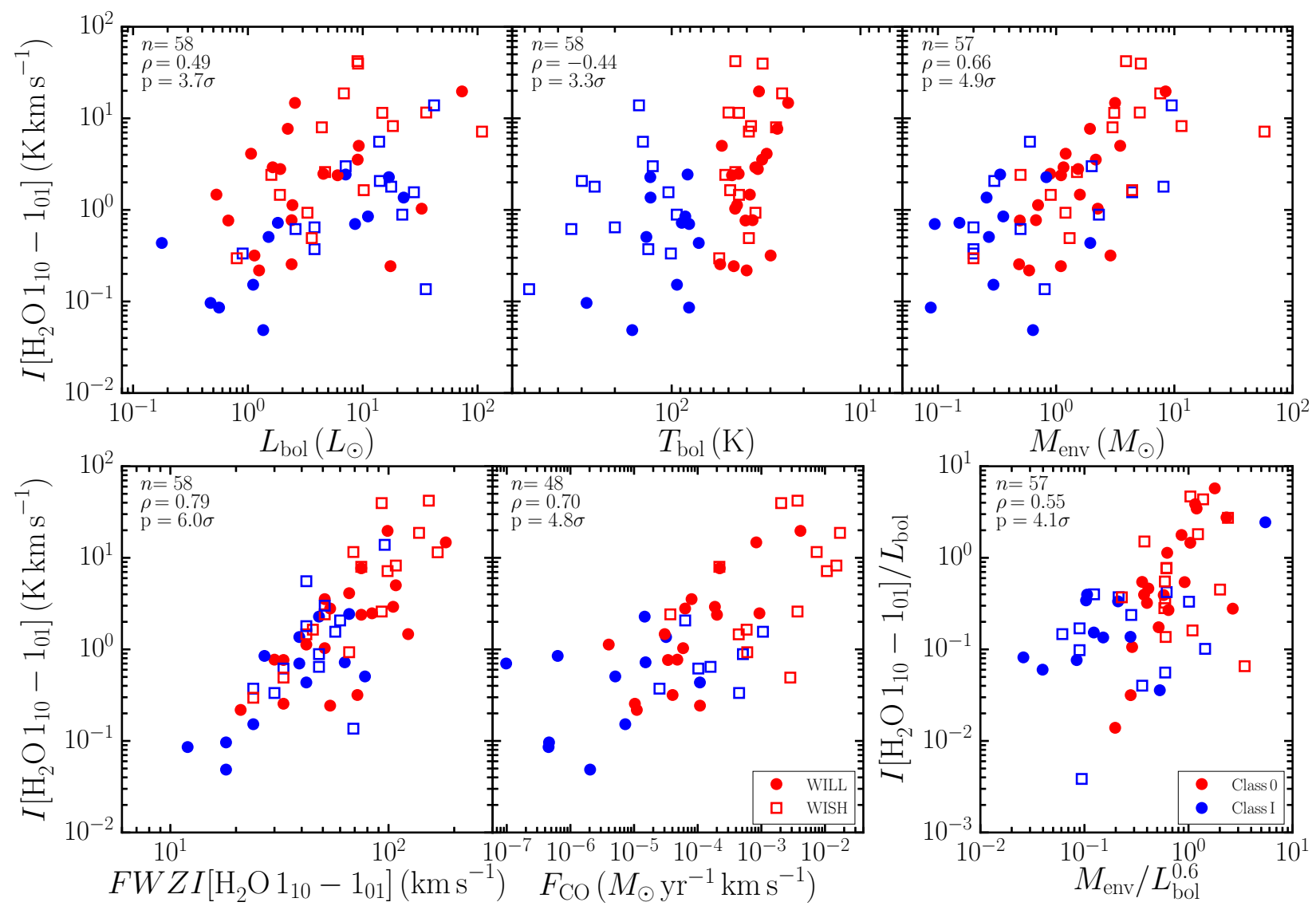

Fig. 8. Comparison of the integrated intensity of $\mathrm{H}_{2} \mathrm{O} 1_{10}-1_{01}$ linearly scaled to a distance of $200 \mathrm{pc}$ with various source (top) and outflow-related (bottom left and middle) properties, as well as the integrated intensity normalised by $L_{\mathrm{bol}}$ vs. the evolutionary indicator $M_{\mathrm{env}} / L_{\mathrm{bol}}^{0.6}($ bottom right) proposed by Bontemps et al. (1996). The number of sources, correlation coefficient and probability that the correlation is not just due to random distributions in the variables are shown in the upper-left of each panel.

found by Kristensen et al. (2012) for the WISH sample alone between the integrated intensity of the water line with its FWZI (at $6.0 \sigma)$ and $M_{\text {env }}(4.9 \sigma)$. A new correlation is also found with $F_{\mathrm{CO}}$ $(4.8 \sigma)$, firmly showing that water emission is related to, though not tracing the same material as, the entrained molecular outflow. Furthermore, shallower trends of water line intensity with $L_{\text {bol }}$ and inversely with $T_{\text {bol }}$, hinted at but not significant in the WISH sample (e.g. see Fig. 6 of Kristensen et al. 2012), are now confirmed as statistically significant at 3.7 and $3.3 \sigma$ respectively.
To further examine the variation of water emission with source evolution, the lower-right panel of Fig. 8 shows the integrated intensity normalised by the source bolometric luminosity (thus minimising the contribution due to source brightness) vs. $M_{\text {env }} / L_{\text {bol }}^{0.6}$, which was proposed by Bontemps et al. (1996) as an evolutionary indicator. The clear positive correlation $(4.1 \sigma)$ seen in this panel reinforces the finding that the intensity of water emission decreases as sources evolve, independent of the relationship between integrated intensity and $L_{\mathrm{bol}}$. 
The WILL observations therefore reinforce and confirm the results from WISH: $\mathrm{H}_{2} \mathrm{O}$ traces a warmer and faster component of protostellar outflow than the cold entrained molecular outflowing material traced by low-J CO (e.g. Nisini et al. 2010; Kristensen et al. 2012; Karska et al. 2013; Santangelo et al. 2013; Busquet et al. 2014; Mottram et al. 2014; San José-García et al. 2016). In addition, they confirm that the intensity of $\mathrm{H}_{2} \mathrm{O}$ is related to envelope mass and the strength of the entrained molecular outflow, and is higher for younger and/or more luminous sources.

\section{3. $[O I]$ emission}

It has been suggested for some time that emission from [O I] is a good alternative tracer of the mass loss from protostellar systems (e.g. Hollenbach 1985; Giannini et al. 2001). In Class 0/I protostars it is thought to primarily trace the atomic/ionised wind, because most PACS observations are spectrally unresolved and those few sources that do show velocity-resolved emission (e.g. see Nisini et al. 2015) are dominated by the unresolved $\left(\lesssim 100 \mathrm{~km} \mathrm{~s}^{-1}\right.$ ) component. While there may be a contribution on-source from the disk, as in more evolved sources (see e.g Howard et al. 2013), [O I] emission in Class 0/I sources is often spatially extended and only fainter off-source by a factor of $\sim 2$ compared to the peak position, so the wind likely still dominates.

The first comprehensive surveys of the [O I] $63 \mu \mathrm{m}$ transition towards samples of YSOs, observed in an 80" beam with the Infrared Space Observatory Long Wavelength Spectrometer (ISOLWS, Swinyard et al. 1998), suggested a link between the mass loss in [O I] and that in $\mathrm{CO}$ for Class 0 sources (Giannini et al. 2001). Only a marginal difference was seen in [O I] luminosity between Class 0 and I sources (Nisini et al. 2002), in contrast to the trend in CO. More recent studies by Podio et al. (2012) and Watson et al. (2016) with PACS on Herschel at 9" resolution have used [O I] observations to claim trends of decreasing mass loss in the wind between Classes 0, I and II. However, both suffered from low number statistics, and Podio et al. (2012) mixed the same ISO results where no trend was found with early detections from Herschel PACS, which have significantly different beam sizes and observing methods that could induce such changes. For example, the chopping as part of PACS observations can cancel out up to $80 \%$ of the large-scale emission that is still detected by ISO (see Appendix E of Karska et al. 2013). The combined WILL, WISH and DIGIT dataset, with consistent observations of a large number of YSOs is ideally placed to help solve this issue.

Figure 9 shows the distribution of [O I] luminosity in the $63 \mu \mathrm{m}$ line, integrated over the PACS spaxels associated with source outflows, and how this varies with various source parameters for the WILL, WISH and DIGIT samples (see Table A.7 for $L[\mathrm{OI}]$ values). Also shown are the measurements from Herschel studies of a number of Class I/II sources in Taurus (Podio et al. 2012), the "Herschel Orion Protostars" survey (HOPS Watson et al. 2016), and the "FU Orionis Objects Surveyed with Herschel" survey (FOOSH Green et al. 2013b) which targeted a number of Flat spectrum and Class II sources that show evidence of FU Ori-type luminosity outbursts. None of the detected sources have a line luminosity below the upper limit for disk sources found by Howard et al. (2013) towards sources in Taurus $\left(4 \times 10^{17} \mathrm{~W} \mathrm{~m}^{-2}\right.$, corresponding to $\sim 2 \times 10^{-5} L_{\odot}$ assuming a distance of $140 \mathrm{pc}$ ).

Two primary results stand out from Fig. 9. First, $L[\mathrm{OI}]$ is strongly correlated with $L_{\text {bol }}$ but not with $M_{\text {env }}$, with sources of all evolutionary classification following the overall trend. This is essentially the reverse of the situation found with low- $J \mathrm{CO}$, where the correlation is weak with $L_{\text {bol }}$ and strong with $M_{\text {env }}$ (see e.g. Fig. 5). $\mathrm{H}_{2} \mathrm{O}$ shows clear correlations with both $M_{\text {env }}$ and $L_{\text {bol }}$ (see 8), though the relationship is slightly stronger with $M_{\text {env }}$ than $L_{\mathrm{bol}}$, consistent with it tracing actively shocked outflow material between the entrained outflow, probed by low- $J$ $\mathrm{CO}$, and the wind, probed by [O I].

Second, there is no statistically significant variation in $L[\mathrm{O} \mathrm{I}]$ with evolutionary stage, either when considering the flat distribution between $L[\mathrm{OI}]$ and $T_{\text {bol }}$ or the histogram of $L[\mathrm{OI}]$, which shows remarkably similar distributions for Class 0 , I or II sources. This is not due to an evolutionary trend being masked by the correlation with $L_{\mathrm{bol}}$, as shown by the flat distribution in $L[\mathrm{OI}] / L_{\text {bol }}$ vs. $M_{\text {env }} / L_{\text {bol }}^{0.6}$. There is also no statistically significant correlation with envelope mass or integrated intensity in the $\mathrm{H}_{2} \mathrm{O} 1_{10}-1_{01}$, which is dominated by the fast, actively shocked component of the molecular outflow.

This apparent contradiction between the evolutionary behaviour of mass-loss indicators, that is, the decrease of $\mathrm{CO}$ and $\mathrm{H}_{2} \mathrm{O}$ velocity, intensity etc. as sources evolve compared to the invariance of [OI], will be explored and discussed in more detail in the following subsections. It is interesting to note that the FOOSH sources, which are all known to be undergoing luminosity outbursts, are on the upper end of, but consistent with, the distribution of other sources in terms of $L[\mathrm{OI}]$ vs. $L_{\mathrm{bol}}$. Thus, [O I] must react relatively quickly to variations in the mass accretion rate, which has a significant contribution to the observed source luminosity.

\subsection{Mass accretion vs. loss}

The balance of mass loss vs. accretion is important in revealing the rate at which the central protostar gains mass, as well as what fraction of the initial envelope will become part of the central source, that is, the core to star efficiency of star formation.

Direct measurement of the mass accretion rate is extremely challenging for embedded protostars because the UV, optical and near-IR continuum and lines typically used to do this in more evolved T-Tauri stars (e.g. Ingleby et al. 2013) are too heavily extincted. An approximate estimate can be obtained, however, by rearranging the equation for accretion luminosity, that is,

$\dot{M}_{\mathrm{acc}}=\frac{L_{\mathrm{acc}} R_{*}}{G M_{*}}$

with the aid of a number of empirically constrained assumptions.

Firstly, accretion is assumed to generate all the observed bolometric luminosity for Class 0 sources and $50 \%$ for Class I sources, in keeping with the range observed in the few cases where this could be measured $\left(L_{\mathrm{acc}} / L_{\mathrm{bol}}=0.1-0.8\right.$ : Nisini et al. 2005; Antoniucci et al. 2008; Caratti o Garatti et al. 2012). Next, a typical stellar mass $\left(M_{*}\right)$ of $0.5 M_{\odot}$ for Class I sources is assumed and $0.2 M_{\odot}$ for Class 0 sources as they are still gaining mass, as in Nisini et al. (2015). The chosen values are for sources that will end up slightly more massive than the peak of the IMF ( 0.2 $M_{\odot}$, Chabrier 2005). However, as already discussed in Sect. 2.2, our sample is biased towards slightly higher luminosities, and thus presumably stellar masses, than the global distribution, so this assumption is probably not far off. Indeed, these stellar masses are broadly in keeping with several recent mass determinations for similar embedded protostars from disk studies (Tobin et al. 2012; Murillo et al. 2013; Harsono et al. 2014; Codella et al. 2014). Finally, we assume a stellar radius $\left(R_{*}\right)$ of $4 R_{\odot}$. The calculated values are given in Table A.7 and shown vs. 
J. C. Mottram et al.: Outflows, infall and evolution of a sample of embedded low-mass protostars
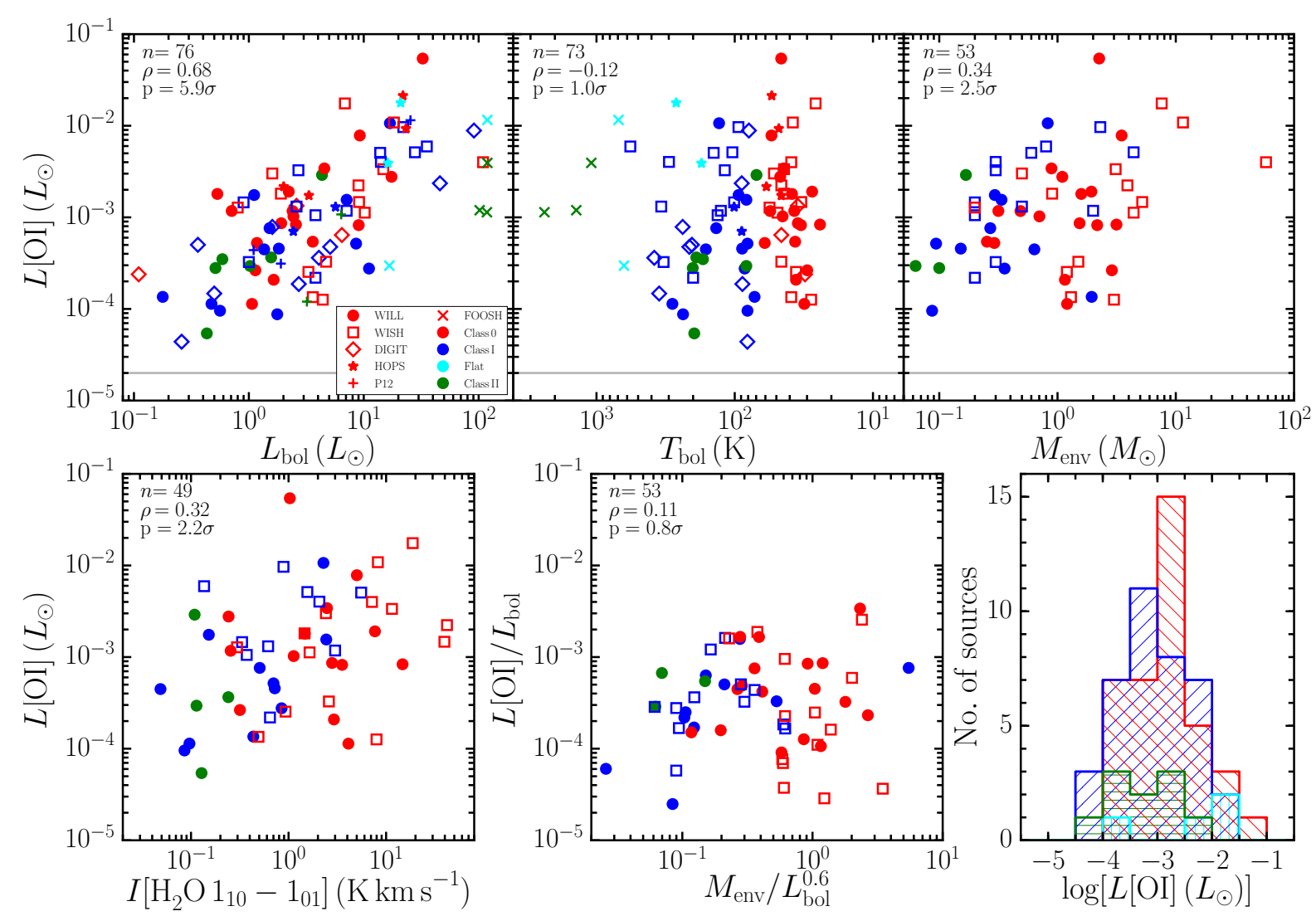

Fig. 9. [O I] luminosity vs. $L_{\text {bol }}$ (top-left), $T_{\text {bol }}$ (top-middle), $M_{\text {env }}$ (top-right) and integrated intensity in the $\mathrm{H}_{2} \mathrm{O} 1_{10}-1_{01}$ transition (bottom-left). Bottom-middle: [OI] luminosity normalised by $L_{\mathrm{bol}}$ vs. the evolutionary indicator $M_{\text {env }} / L_{\mathrm{bol}}^{0.6}$ proposed by Bontemps et al. (1996). The plots include data from the WILL, WISH and DIGIT samples, as well as from the literature from Podio et al. (2012) and the HOPS (Watson et al. 2016) and FOOSH (Green et al. 2013b) surveys where available. The number of sources and correlation statistics in the upper-right of each panel include only Class 0/I sources so as to be conservative. The horizontal grey line in the top panels indicates the upper limit for disk emission from Howard et al. (2013). Bottom-right: histogram of $L[\mathrm{OI}]$ as a function of spectral type, including sources from all surveys.

$L_{\mathrm{bol}}, T_{\mathrm{bol}}$ and $M_{\mathrm{env}}$ in the top panels of Fig. 10. The solid line in the upper-left panel shows the relation assumed in the evolutionary models of Duarte-Cabral et al. (2013), that is, Eq. (2) with $\tau=3 \times 10^{5} \mathrm{yr}$.

Hollenbach (1985) noted a simple scaling between the [O I] line luminosity at $63 \mu \mathrm{m}$ and the total mass-flux through the dissociative shock(s) producing it, given by:

$\dot{M}_{\mathrm{s}}=10^{-4} L[$ O I $63 \mu \mathrm{m}]$.

For shocks generated by the wind, as is most likely the case for the emission probed by [O I], the mass flux through the shock(s), $\dot{M}_{\mathrm{s}}$, is related to the wind mass-flux, $\dot{M}_{\mathrm{w}}$, by the general formula (see Dougados et al. 2010):

$\dot{M}_{\mathrm{s}}=N_{\mathrm{s}} \frac{v_{\mathrm{s}}}{v_{\mathrm{w}} \cos (\theta)} \dot{M}_{\mathrm{w}}$,

where $N_{\mathrm{s}}$ is the number of shocks in the beam, $v_{\mathrm{s}}$ is the shock speed, and $\theta$ is the angle between the normal to the shock front and the wind direction (the $1 / \cos (\theta)$ term then accounts for the ratio of the shock area to the wind cross section). It may be seen that $\dot{M}_{\mathrm{s}}=\dot{M}_{\mathrm{w}}$ in the simple case considered by Hollenbach (1985) if we are observing a static terminal shock where the wind is stopped against a much denser ambient medium; in this case, $N_{\mathrm{s}}=1$ and $v_{\mathrm{s}}=v_{\mathrm{w}} \cos (\theta)$. This remains valid if the wind is not isotropic but collimated into a jet.

If we are instead observing weaker internal shocks travelling along the jet/wind, then $v_{\mathrm{s}} \ll v_{\mathrm{w}} \cos (\theta)$ but this will tend to be compensated for by the presence of several shocks in the beam (i.e. $N_{\mathrm{s}}>1$ ), as suggested by the chains of closely spaced internal knots seen in optical jets.

An alternative method for obtaining the average $\dot{M}_{\mathrm{w}}$ in this case is to consider that the [O I] emission is approximately uniform along the flow within the aperture, and to divide the emitting gas mass by the aperture crossing time. The derivation of emitting mass requires assumptions on the temperature and electron density, which are somewhat uncertain without also having observations of the [OI] $145 \mu \mathrm{m}$ transition. However, Nisini et al. (2015) found that the differences are small between this alternative per-unit-length calculation and the Hollenbach (1985) formulation for a terminal static wind shock (i.e. $\dot{M}_{\mathrm{s}}=$ $\left.\dot{M}_{\mathrm{w}}\right)$. Hence, although we note that there are some uncertainties involved, we adopt $\dot{M}_{\mathrm{w}}[\mathrm{O} \mathrm{I}]=\dot{M}_{\mathrm{s}}$ as given by Eq. (7) to estimate the wind mass-flux from $L[\mathrm{OI}]$ for our targets. The calculated values are given in Table A.7.

The ratio of the mass-loss rate in the wind as measured from [O I] using Eq. (7) to the mass accretion rate (i.e. $\dot{M}_{\mathrm{w}}[\mathrm{O}$ I $] / \dot{M}_{\text {acc }}$ ) is compared to $L_{\mathrm{bol}}, T_{\mathrm{bol}}$ and $M_{\mathrm{env}}$ in the middle panels of Fig. 10. $\dot{M}_{\mathrm{w}}[\mathrm{O} \mathrm{I}] / \dot{M}_{\text {acc }}$ varies from approximately $0.1 \%$ to $100 \%$ with a median of $13 \%$, in agreement with previous determinations (e.g. Cabrit 2009; Ellerbroek et al. 2013) and in line with theoretical predictions (e.g. Konigl \& Pudritz 2000; Ferreira et al. 2006). However, approximately two-thirds of all Class 0 sources lie below $10 \%$. 


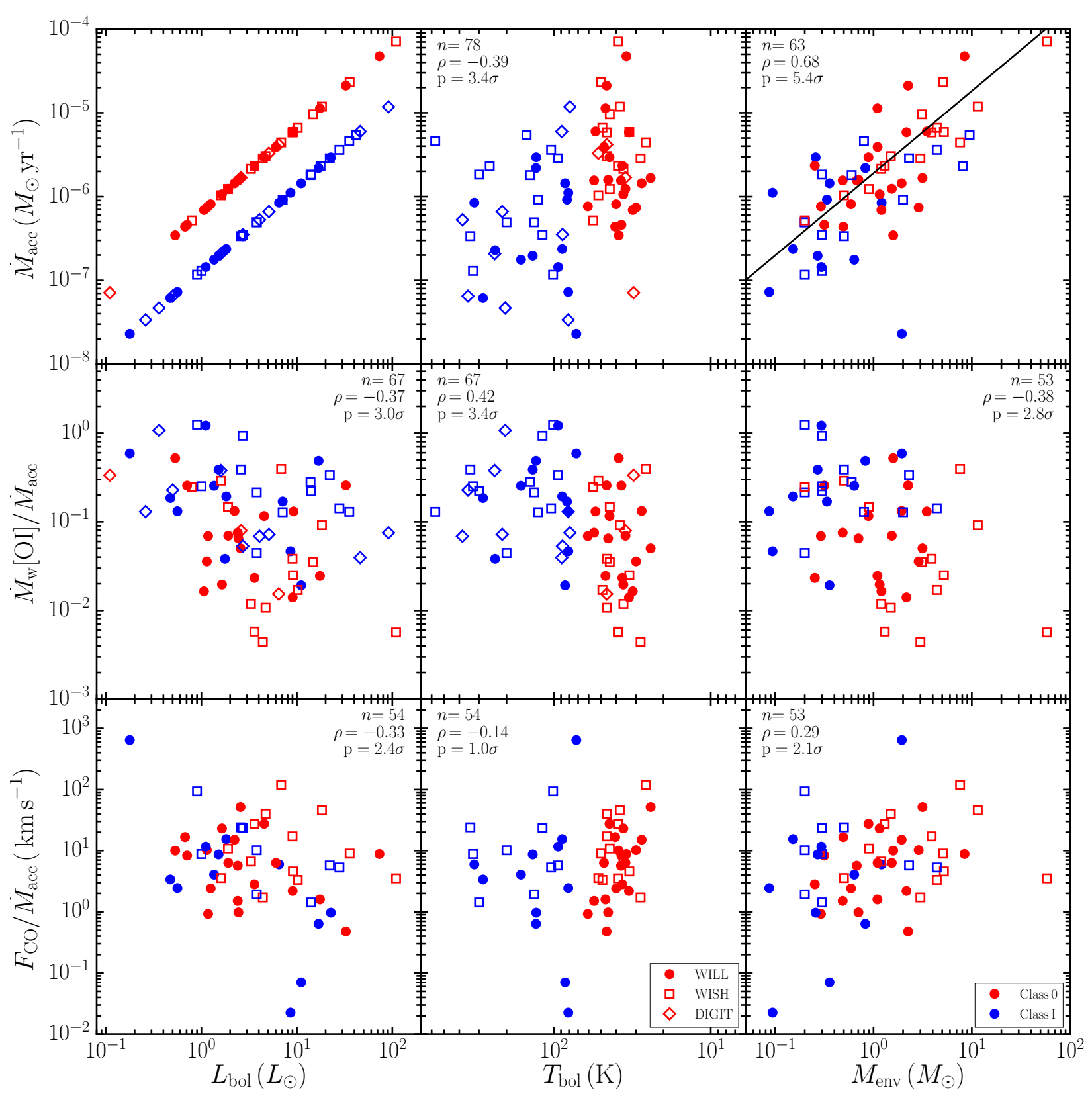

Fig. 10. Mass accretion rate $\left(\dot{M}_{\text {acc }}\right.$, top), the ratio of mass-loss rate in the wind from [O I] to mass accretion rate (middle), and the ratio of outflow force from CO $J=3-2$ to mass accretion rate (bottom), vs. $L_{\mathrm{bol}}$ (left), $T_{\mathrm{bol}}$ (middle) and $M_{\mathrm{env}}$ (right). The solid line in the upper-right panel indicates the relationship between $\dot{M}_{\text {acc }}$ and $M_{\text {env }}$ from Duarte-Cabral et al. (2013), which is part of the evolutionary models shown in Fig. 1 .

The lower panels of Fig. 10 show similar comparison using the outflow force as measured from CO $J=3-2$. Assuming the entrainment process is momentum conserving:

$F_{\mathrm{CO}}=\dot{M}_{\mathrm{w}} v_{\mathrm{w}} \epsilon_{\mathrm{ent}}$,

where $\epsilon_{\mathrm{ent}}$ is the entrainment efficiency. The ratio with the mass accretion rate is then:

$\frac{F_{\mathrm{CO}}}{\dot{M}_{\mathrm{acc}}}=\frac{\dot{M}_{\mathrm{w}}}{\dot{M}_{\mathrm{acc}}} v_{\mathrm{w}} \epsilon_{\mathrm{ent}}$.

$\frac{\dot{M}_{\mathrm{w}}}{\dot{M}_{\mathrm{acc}}} v_{\mathrm{w}}$ is expected to be approximately constant due to conservation of angular momentum, with a value close to the Keplerian velocity of the disk at the launching radius (Duarte-Cabral et al. 2013).
We find that $F_{\mathrm{CO}} / \dot{M}_{\mathrm{acc}}$ is relatively invariant with $L_{\mathrm{bol}}, T_{\mathrm{bol}}$ and $M_{\text {env }}$, as shown by values of the Pearson coefficient $\rho$ consistent with 0 (i.e. $p<3 \sigma$ ). Taken together, this suggests that the efficiency of entrainment, $\epsilon_{\text {ent }}$, is not dependent on source properties. The Keplerian velocity for a disk around a 0.2 or $0.5 M_{\odot}$ source is approximately $10-20 \mathrm{~km} \mathrm{~s}^{-1}$ at $1 \mathrm{AU}$, which, for a median value of $F_{\mathrm{CO}} / \dot{M}_{\mathrm{acc}}$ of $6.3 \mathrm{~km} \mathrm{~s}^{-1}$, suggests values for $\epsilon_{\mathrm{ent}}$ of approximately $0.3-0.6$. If the wind is launched at larger radii then $\epsilon_{\text {ent }}$ could be closer to 1 .

$L$ [O I] does not vary with $T_{\text {bol }}, M_{\text {env }}$ or evolutionary stage (see Sect. 4.3 and Fig. 9), so the increase of $\dot{M}_{\mathrm{w}}[\mathrm{O} \mathrm{I}] / \dot{M}_{\text {acc }}$ between Class 0 and I with increasing $T_{\text {bol }}$ and with decreasing $M_{\text {env }}$ is caused by the decrease in $\dot{M}_{\text {acc }}$, while $\dot{M}_{\mathrm{w}}[\mathrm{O}$ I] remains relatively constant. In contrast, the invariance of $F_{\mathrm{CO}} / \dot{M}_{\mathrm{acc}}$ is caused by both $\dot{M}_{\text {acc }}$ and $\dot{M}_{\mathrm{w}}$ decreasing with increasing $T_{\text {bol }}$ and decreasing $M_{\text {env }}$ (see the lower panels of Fig. 5 for variation of 


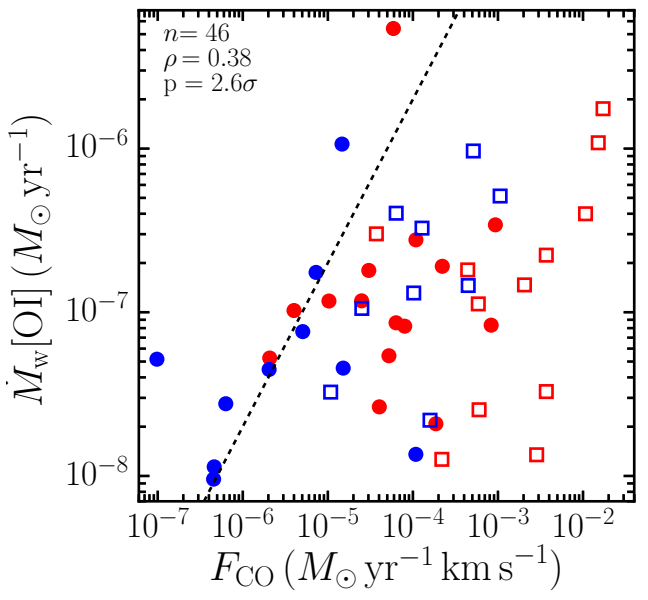

Fig. 11. Ratio of mass-loss rate in the wind from [O I] to the outflow force from $\mathrm{CO} J=3-2$. The dashed line indicates the expected locus if both trace the mass-loss rate in the wind, $v_{\mathrm{w}}=100 \mathrm{~km} \mathrm{~s}^{-1}$ and $\epsilon_{\mathrm{ent}}=0.5$. Lower values of $v_{\mathrm{w}}$ and/or $\epsilon_{\text {ent }}$ move this line to the left. The symbols and colours have the same meaning as in Fig. 10.

$F_{\mathrm{CO}}$ with $T_{\text {bol }}$ and $\left.M_{\text {env }}\right)$. The reason for the difference in behaviour between these two measures of the ratio of mass loss to mass accretion is discussed further in the following section.

\subsection{On the difference between [O I] and $\mathrm{CO}$}

The difference in behaviour between the atomic component of the wind (as traced by [OI]) and the entrained molecular outflow (as traced by low- $J$ CO) might seem to be in contradiction with models where the wind is the driving agent of the outflow (see e.g. Arce et al. 2007). Indeed a direct comparison, shown in Fig. 11, suggests that either the wind and outflow are not linked, $[\mathrm{OI}]$ is under-estimating the mass loss rate in the wind or $F_{\mathrm{CO}}$ is overestimated. However, there are several factors relating to what component of the system each tracer probes that argue against rushing to such a conclusion.

First, [OI] only traces the atomic component of the wind and/or jet. Jets in Class 0 protostars are known to have a significant molecular component, as identified from high-velocity features (detected in e.g. $\mathrm{CO}, \mathrm{SiO}$ and/or $\mathrm{H}_{2} \mathrm{O}$, Bachiller et al. 1991; Tafalla et al. 2010; Kristensen et al. 2011) with typical mass-loss rates of approximately $10^{-7}-10^{-5} M_{\odot} \mathrm{yr}^{-1}$ (e.g. Santiago-García et al. 2009; Lee et al. 2010a). These are typically approximately ten times higher than measured from [O I], but the molecular jet component disappears in older sources. This suggests an evolution in composition from molecular to atomic/ionised (see Nisini et al. 2015, for a detailed discussion), most likely due to increasing temperature of the protostar and decreasing density, and thus shielding, in the jet. Such arguments also hold for any wide-angle wind that could be present and contributing to driving the entrained $\mathrm{CO}$ outflow. Therefore, while the mass-loss rate due to the wind as a whole will decrease as the source evolves, in line with the decrease in the average mass accretion rate, the mass loss in the atomic component may remain approximately constant due to the shift in the composition of the wind.

Next, the optical depth of the continuum at $63 \mu \mathrm{m}$ is likely considerable in the inner envelope in Class 0 sources (see e.g. Kristensen et al. 2012), so the observed [O I] flux may be significantly lower than the "true" emission. The continuum optical depth will decrease as the source evolves and $M_{\text {env }}$ decreases, which may also act to counteract the evolution in the mass loss in the wind. However, such an effect should also cause the ratio of the $63 \mu \mathrm{m}$ to $145 \mu \mathrm{m}$ [O I] lines to vary with continuum optical depth of the source, and a wavelength-dependent deficit in $\mathrm{CO}$ and $\mathrm{H}_{2} \mathrm{O}$ transitions. Neither is clearly seen in PACS observations (see e.g. Karska et al. 2013). This is therefore likely a minor effect dominating only for sight-lines directly towards the protostar through the disk.

Finally, there is increasing evidence that episodic or timevariable accretion is important in embedded protostars from the very earliest phases of their evolution (see Dunham et al. 2014; Audard et al. 2014, for recent reviews). Accretion variability provides a consistent explanation for very low luminosity objects (e.g. Dunham et al. 2006), the observed spread and trends in protostellar (e.g. Dunham et al. 2010) and outflow related (e.g. Duarte-Cabral et al. 2013) properties, and luminosity bursts, brighter by at least a factor of ten, have now been observed in at least two embedded sources (Caratti o Garatti et al. 2011; Fischer et al. 2012; Safron et al. 2015). Chains of high-velocity molecular knots or "bullets" observed in Class 0 outflows and jets, with typical spacings of 1000-10000 AU between minor and major episodes, respectively (e.g. Santiago-García et al. 2009; Lee et al. 2015), past heating of $\mathrm{CO}_{2}$ ice (e.g. Kim et al. 2012) and the difference between the expected and observed CO snow surface in a number of protostars (Visser et al. 2015; Jørgensen et al. 2015) also provide indirect evidence of outbursts.

The imprint of time-variable accretion will be different for the molecular outflow and atomic wind, leading to differences in their properties. The luminosity will react quickly to any changes in the accretion rate (Johnstone et al. 2013), and thus traces the current or instantaneous activity. Since $[\mathrm{O} \mathrm{I}]$ is dominated by the wind, it traces material that is closely related to the current accretion state and thus is correlated with luminosity regardless of whether the source is in outburst (e.g. the FOOSH sources) or not (see Fig. 9).

In contrast, the entrained molecular outflow traced by low$J$ CO, particularly when measured over the full extent of the outflow, is an average of the ejection activity over at least $10^{3}-10^{5} \mathrm{yr}$. Indeed, the highest intensity in the entrained outflow as traced by low- $J$ CO is usually offset from the central source. If these spots represent major ejections triggered by accretion bursts, then such episodes should have occurred approximately hundreds to thousands of years ago, leaving enough time for the luminosity and circumstellar material to cool back to pre-burst levels (e.g. Arce \& Goodman 2001; Arce et al. 2013). Thus, the mass loss in the molecular outflow is related to the time-averaged mass-accretion rate and may be dominated by any periods of high accretion/ejection during outbursts (see also e.g. Dunham et al. 2006; Lee et al. 2010b).

The decrease of $F_{\mathrm{CO}}$ between Class 0 and I (see Fig. 5) therefore shows that the average mass accretion rate decreases as sources evolve, as originally proposed by Bontemps et al. (1996). The combination of decreasing mass accretion rates and episodic accretion was shown by Duarte-Cabral et al. (2013) to be consistent with the observed relationships between, and spread of, $L_{\mathrm{bol}}, M_{\mathrm{env}}$ and $F_{\mathrm{CO}}$. In particular, variation of the mass-accretion rate on shorter timescales than the dynamical timescale of the outflow helps to explain why outflow properties are less correlated with $L_{\text {bol }}$ than with $M_{\text {env }}$ (see Fig. 5 and Sect. 4.1). Those sources that show particularly high outflow forces and/or peak emission close to the source position may therefore have recently finished such a burst, or have a higher duty cycle of outburst to quiescent accretion. Thus, the mass-loss 

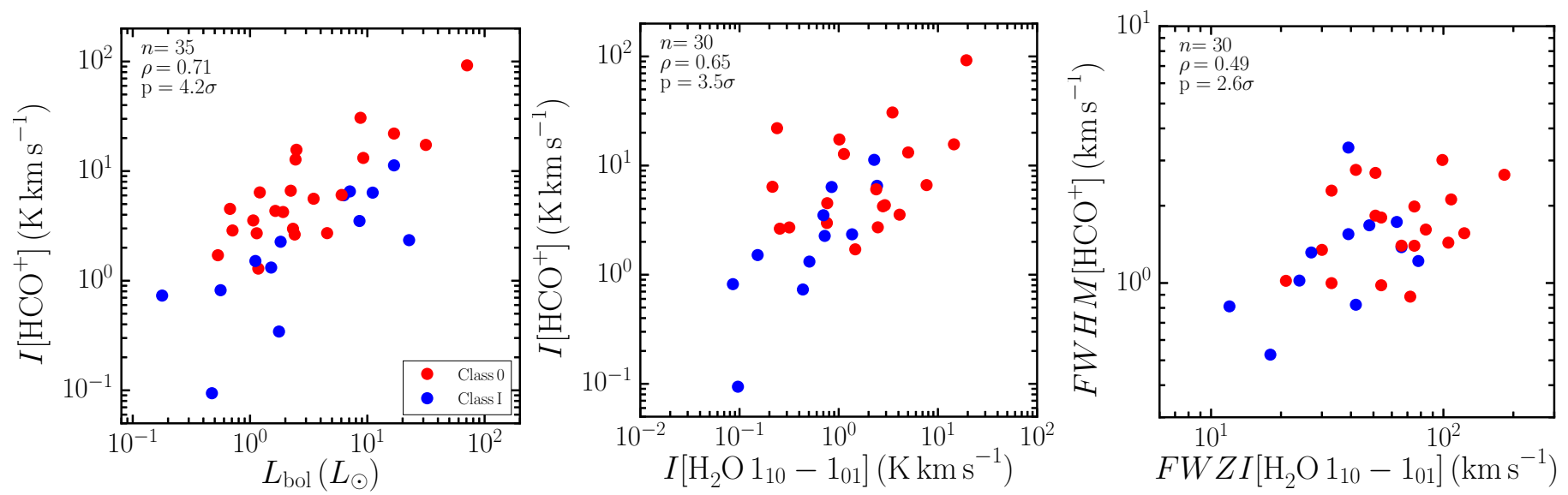

Fig. 12. Comparison of the integrated intensity of $\mathrm{HCO}^{+} J=4-3$ with $L_{\mathrm{bol}}$ (left) and the integrated intensity of $\mathrm{H}_{2} \mathrm{O} 1_{10}-1_{01}$ scaled to a distance of $200 \mathrm{pc}$ (middle), as well as the FWHM of $\mathrm{HCO}^{+}$vs. the FWZI of $\mathrm{H}_{2} \mathrm{O} 1_{10}-1_{01}$ (right). The number of sources, correlation coefficient and probability that the correlation is not just due to random distributions in the variables are shown in the upper-left of each panel.

rate in the wind from $[\mathrm{OI}]$ and in the outflow force measured by low- $J \mathrm{CO}$ are not directly correlated because the relationship between the current and time-averaged mass-accretion rate will be different for each source based on a complex combination of the source age, properties and mass-accretion history.

Some combination of the effects discussed above therefore causes the observed lack of correlation between $\mathrm{CO}$ and [O I]. As such, [O I] is not necessarily a direct alternative to $\mathrm{CO}$ for tracing mass loss and/or entrainment due to the jet/wind/outflow system in protostars, in contradiction to the early findings of Giannini et al. (2001).

\section{Envelope}

\section{1. $\mathrm{HCO}^{+}$vs. $\mathrm{H}_{2} \mathrm{O}$}

Instead of outflows, $\mathrm{HCO}^{+} J=4-3$ emission primarily traces cool, high-density envelope material $\left(T \sim 40 \mathrm{~K}, n_{\mathrm{cr}}=2 \times\right.$ $10^{7} \mathrm{~cm}^{-3}$ though the effective density for optically thick emission could be as low as $10^{4} \mathrm{~cm}^{-3}$, see Shirley 2015), and so is a relatively clean discriminator between young, embedded protostars and pre-stellar or more evolved disk sources (van Kempen et al. 2009; Carney et al. 2016). Spatially compact detections are found in this line towards most of the WILL sample sources, confirming them to be genuine embedded Class 0/I sources, while pre-stellar and Class II sources are either nondetections or show extended emission with no clear peak at the source position (see Appendix A.7 for details).

$\mathrm{H}_{2} \mathrm{O}$ emission is a good tracer of warm, relatively dense material in shocks related to protostellar outflows $(T \gtrsim 300 \mathrm{~K}$, $n=10^{5}-10^{8} \mathrm{~cm}^{-3}$, Kristensen et al. 2013; Mottram et al. 2014). Sources with higher luminosities typically have stronger outflows (and thus stronger $\mathrm{H}_{2} \mathrm{O}$ emission) and will lead, all other things being equal, to more mass at a given temperature in their envelopes and thus higher intensity in molecular tracers such as $\mathrm{HCO}^{+}$. It is therefore not unreasonable to expect that the emission in these two tracers may be related in Class 0/I sources.

Carney et al. (2016) compared the nature of $\mathrm{HCO}^{+}$emission (compact, confused or extended/not detected) and the detection of water in the WILL PACS $179 \mu$ m observations. There are 18 sources in common between their sample and WILL: 13 are classified as Class 0/I (i.e. compact $\mathrm{HCO}^{+} J=4-3$ ), 3 as confused and 2 as Class II (i.e. extended and/or non-detections in $\left.\mathrm{HCO}^{+}\right)$. Most sources $(14 / 18)$ have detected $179 \mu \mathrm{m}$ water emission, with four Class 0/I sources and one confused source showing extended emission. As both Class II sources were detected, while three Class 0/I sources and one confused source was not, Carney et al. (2016) did not find a clear relationship with evolution between the spatial distributions and detection of $\mathrm{HCO}^{+}$and $\mathrm{H}_{2} \mathrm{O}$.

Considering the $\mathrm{H}_{2} \mathrm{O} \quad 1_{10}-1_{01}$ HIFI observations (see Fig. 12), for the two Class II sources, TAU 07 is not detected at the $3 \sigma$ level and TAU 09 shows very weak, narrow emission with an integrated intensity of $0.16 \mathrm{~K} \mathrm{~km} \mathrm{~s}^{-1}$, equivalent to $10^{-18} \mathrm{~W} \mathrm{~m}^{-2}$, which could include a contribution from the disk (cf. Podio et al. 2012, 2013; Fedele et al. 2013). Thus, while $\mathrm{H}_{2} \mathrm{O}$ may be detected in either Class 0/I or II, the origin and intensity of the water emission changes as the source evolves. Strong detection of either $\mathrm{HCO}^{+}$or water is therefore still a good indication of the youth of a protostar.

For the Class 0/I sources, the full WILL dataset enables the relationship between these species to be probed further by comparing the integrated intensity and FWHM of $\mathrm{HCO}^{+}$ with the integrated intensity and FWZI of the $\mathrm{H}_{2} \mathrm{O} 1_{10}-1_{01}$ HIFI observations (see Fig. 12). $\mathrm{HCO}^{+}$intensity is correlated both with $L_{\text {bol }}$ and the intensity of the water line at 4.2 and $3.5 \sigma$ significance, respectively. There is not a strong, statistically significant relationship between the kinematics of the two lines and no significant line-wings are seen in the $\mathrm{HCO}^{+}$spectra (see Fig. A.3). $\mathrm{HCO}^{+} J=4-3$ therefore seems to primarily trace parts of the envelope that are further from, and thus less disturbed by, the outflow. $\mathrm{HCO}^{+}$can be destroyed through reactions with $\mathrm{H}_{2} \mathrm{O}$ (e.g. Jørgensen et al. 2013), so the higher abundance of $\mathrm{H}_{2} \mathrm{O}$ in the outflow $\left(X\left[\mathrm{H}_{2} \mathrm{O}\right]\right.$ or approximately $10^{-5}-10^{-7}$ : Tafalla et al. 2013; Santangelo et al. 2013; Kristensen et al. 2017) compared to the envelope $\left(10^{-8}-10^{-11}\right.$ : Mottram et al. 2013; Schmalzl et al. 2014) could be suppressing the $\mathrm{HCO}^{+}$abundance in the outflow. This would explain why it is a poorer tracer of the outflow than might otherwise be expected. The correlation between the intensity of the two lines is therefore likely due to the relation between emission in each line and the source luminosity and structure, assisted by their tracing similar densities.

\subsection{Infall signatures}

The bulk of the $\mathrm{H}_{2} \mathrm{O}$ emission comes from outflow-related shocks that have Gaussian-like profiles in velocity-resolved spectra. Once this contribution is removed, the residual profiles 


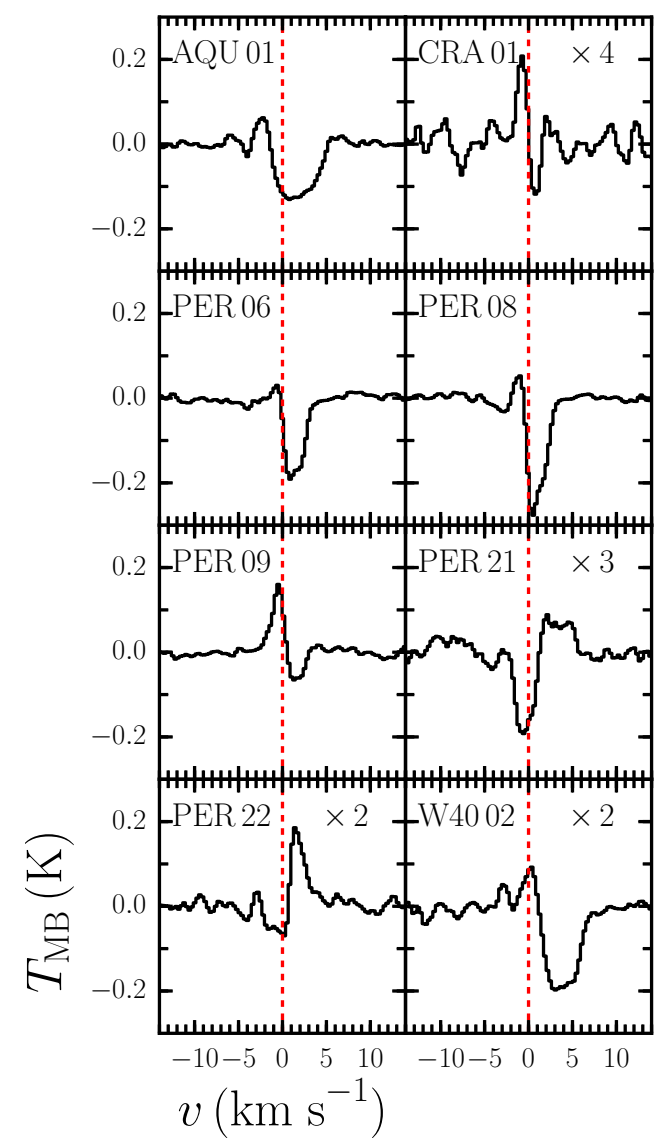

Fig. 13. Outflow-subtracted $\mathrm{H}_{2} \mathrm{O} 1_{10}-1_{01}$ residual line profiles for those sources showing either regular (PER 21 and PER 22) or inverse (all other) P-Cygni line profiles. All have been recentred so that the source velocity is at zero. The number in the upper-right corner of each panel indicates what factor the spectra have been multiplied by to aid visibility.

show the remaining water emission and/or absorption associated with the envelope. For the WISH sample, this process revealed seven sources with inverse P-Cygni line profiles indicative of infall and five with regular P-Cygni line profiles indicative of expansion motions in the envelope (Kristensen et al. 2012; Mottram et al. 2013). When the same procedure is performed for the WILL $\mathrm{H}_{2} \mathrm{O} 1_{10}-1_{01}$ observations, removing the shock emission using the Gaussian decomposition of the profiles from San José-García (2015), six sources (3 Class 0 and 3 Class I) show inverse P-Cygni and two sources (both Class 0) show regular P-Cygni line profiles (see Fig. 13).

The two WILL sources in Serpens South have broad water absorption features, but they are not offset enough to be considered inverse P-Cygni (see Fig. 2). These may trace the large-scale cloud collision identified by Kirk et al. (2013), similar to that in the Serpens Main cloud first identified by Duarte-Cabral et al. (2011) and revealed to be the origin of the strong inverse P-Cygni line profile in water in Serpens-SMM4 by Mottram et al. (2013).

As an optically thick, higher density tracer, $\mathrm{HCO}^{+} J=4-3$ is also sensitive to infall and expansion motions in protostellar envelopes (e.g. Gregersen et al. 1997; Myers et al. 2000). Though single-dish observations do not show absorption below the continuum, they can exhibit asymmetric line profiles, with the peak shifted to either the blue (infall) or red (expansion). The asymmetry between the red and blue peaks can be quantified using

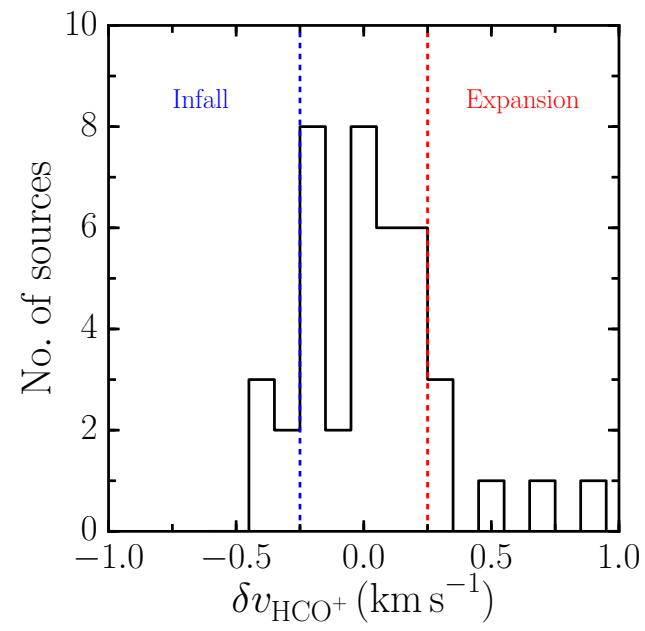

Fig. 14. Histogram of the normalised offset of the peak of $\mathrm{HCO}^{+} 4-3$ with respect to $\mathrm{C}^{18} \mathrm{O} 3-2$. The red and blue dashed lines indicate the boundaries outside which the offset is considered significant (see Mardones et al. 1997).

the $\delta v$ parameterisation suggested by Mardones et al. (1997, see Sect. A.6 for more details):

$\delta v=\frac{v_{\text {thick }}-v_{\text {LSR }}}{F W H M_{\text {thin }}}$,

where $v_{\text {thick }}$ is the velocity of the peak emission in an optically thick tracer (in this case $\mathrm{HCO}^{+}$) and $\mathrm{FWHM}_{\text {thin }}$ is the line width of an optically thin tracer (in this case $\mathrm{C}^{18} \mathrm{O}$ ). Values above or below 0.25 indicate a shift in the optically thick line of more than a quarter of the optically thin line width and so are considered significant. Thus, values above 0.25 indicate expansion motions while those below -0.25 indicate infall. Values between -0.25 and 0.25 are consistent with the optically thick and thin tracers being in agreement. A histogram of the values calculated for the WILL sample is shown in Fig. 14, with five sources showing blue asymmetry (i.e. $\delta v<-0.25,3$ Class 0 and 2 Class I) and six showing red asymmetry (i.e. $\delta v>0.25,3$ Class 0 and 3 Class I).

Only one source (PER 08) exhibits non-static line signatures in both water and $\mathrm{HCO}^{+}$, however they conflict as the water shows an inverse $\mathrm{P}-\mathrm{Cygni}$ line profile and the $\mathrm{HCO}^{+}$a red asymmetry, leaving the status of this source uncertain. It is likely that these two tracers probe different radii, and so perhaps infall and expansion dominate in different parts of the envelope.

What is possibly more puzzling is that the vast majority of sources do not show indications of either infall or expansion in either tracer. Two-sample Kolmogorov-Smirnov (K-S) tests were performed comparing the cumulative distributions of source properties (e.g. $L_{\text {bol }}, T_{\text {bol }}$ etc.) for sources that show infall or expansion motions with respect to those that do not, in order to see if any source properties correlate with the detection of infall or expansion signatures. Only the integrated intensity of $\mathrm{C}^{18} \mathrm{O}$ $3-2$ shows a statistically significant difference $(\leq 1 \%$ chance of being drawn from the same distribution) between sources with either an infall or expansion signature and those that do not: sources with higher $\mathrm{C}^{18} \mathrm{O}$ line intensity are more likely to show signs of infall.

Sources with clear infall motions are statistically more likely to have higher FWZI in ${ }^{12} \mathrm{CO} 3-2$, that is, broader outflow linewings, than those showing no or expansion envelope motions ( $0.9 \%$ likelihood of coming from the same distribution). However, there are some sources that have high FWZI but no indication of radial envelope motions. One other result worth noting is 
that the presence of envelope motions is not more likely for certain values of outflow inclination (see van der Marel et al. 2013, and Sect. A.4 for details of how these were determined), suggesting that the orientation of the protostellar system is not the overriding cause of not detecting infall or expansion in our observations.

Infall must take place in all protostars, at least in the early phases, and at later times it seems unlikely that expansion of the envelope is restricted to a few select sources. Thus the low detection rate of such signatures in both tracers and the lack of a consistent trend with evolutionary Class is puzzling. It may well be that this is an observational effect, caused by small infall motions being lost in the general turbulent field on the large spatial scales that dominate single-dish observations. Mapping the velocity field inside protostellar envelopes with interferometers is likely needed to conclusively understand how material moves radially, and how this varies between different sources and over time (e.g. Yen et al. 2014; Aso et al. 2015; Evans et al. 2015).

\section{Evolution of water line profile components from Class 0 to Class I}

The intensity and line-width of water emission decreases for WISH sources between Class 0 and I (Kristensen et al. 2012; Mottram et al. 2014), while the rotational temperature of mid- $J$ $\mathrm{CO}$ and water excitation conditions do not (Karska et al. 2013; Mottram et al. 2014). Mottram et al. (2014) therefore suggested that the observed evolution in water line intensity and line-width from Class 0 to I was caused by a decrease in the velocity of the wind driving the outflow, due to the increase of the outflow cavity opening angle as proposed in the models of Panoglou et al. (2012), for example, rather than a decrease in density in the $\mathrm{H}_{2} \mathrm{O}$ emitting gas. However, the small sample size of the WISH survey when broken down into evolutionary classifications meant that some trends, while hinted at by the data, were not statistically significant.

Inclusion of the WILL sample helps to resolve this issue. For example, Fig. 15 shows a histogram of the components found in HIFI water spectra, updated from that shown by Kristensen et al. (2012) to include the WILL survey sources and now using the more physically motivated nomenclature introduced by Mottram et al. (2014). A broad cavity shock component is observed in almost all Class 0 and I sources. Mottram et al. (2014) argued that this is caused by C-type shocks in the outflow cavity wall, though Yvart et al. (2016) suggest an alternate explanation where this component is formed in a dusty disk wind. Spot shock components, associated with offset $J$-type shocks either in bullets along the jet or at the base of the outflow (Kristensen et al. 2013; Mottram et al. 2014), are far more likely to be detected in Class 0 than Class I sources. Inverse P-Cygni line profiles associated with infall are more common for younger sources, though the inclusion of the WILL sample means that expansion motions traced by regular P-Cygni line profiles are now approximately equally common in both Class 0 and Class I sources. With the exception of the regular P-Cygni profiles, the evolution of water line profile components found for the combined WISH and WILL samples confirms the conclusion of Kristensen et al. (2012) and Mottram et al. (2014) that the outflows of young Class 0 sources are more energetic and their envelopes more infall-dominated than their more evolved Class I counterparts.

Two-sample K-S tests show that there is less than $2 \%$ chance of the Class 0 and I distributions of $M_{\text {env }}, M_{\text {out }}, F_{\mathrm{CO}}, \dot{M}_{\text {out }}$, the integrated intensity of $\mathrm{HCO}^{+}$and $\mathrm{H}_{2} \mathrm{O} 1_{10}-1_{01}$, and the FWZI of

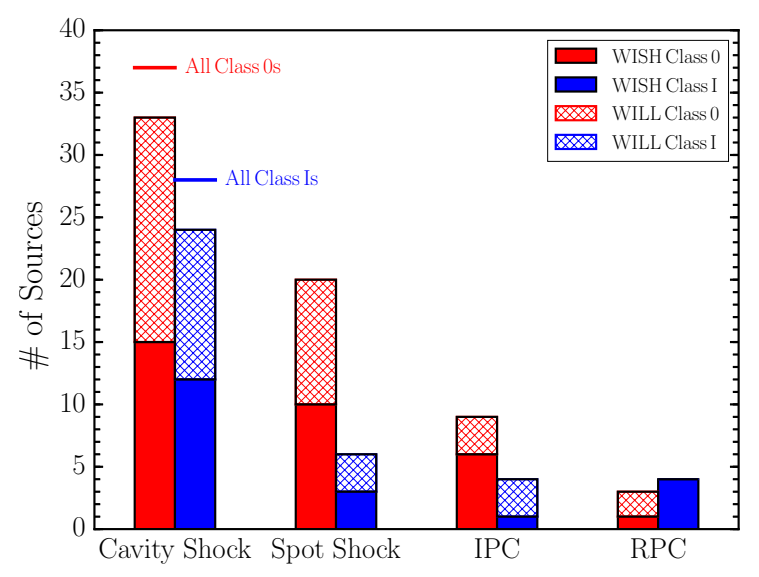

Fig. 15. Bar chart of the number of shock and inverse/regular P-Cygni envelope components seen in water in the WISH (solid) and WILL (hatched) surveys for Class 0 (red) and I (blue) sources. The horizontal red and blue lines indicate the total number of Class 0 and I sources across both samples respectively.

$\mathrm{CO} J=3-2$ and $\mathrm{H}_{2} \mathrm{O} 1_{10}-1_{01}$ being drawn from the same distribution, with the values for Class 0 sources being larger on average than those of Class I sources. In particular, this confirms the decrease in $F_{\mathrm{CO}}$ and both the line-width and intensity of water emission with evolution of the central source, reinforcing the direct relation between water emission and outflow/shock activity.

One caveat is that the Class 0 and I sources in WISH and WILL sources are not evenly drawn from the sampled starforming regions. For example, only Class I or II sources are included in the Taurus star-formation region, while many of the Class 0 sources are in Perseus, which is a much more active starforming complex. The observed differences and trends between Class 0 and I may therefore be accentuated by environmental differences. However, all the evidence suggests that Class I sources have slower, less powerful outflows and show less sign of strong infall motions in their envelopes than Class 0 sources.

\section{Summary and conclusions}

This paper has presented a set of Herschel and ground-based follow-up observations, characterisation and initial analysis of a flux-limited sample of Class 0/I YSOs in the Gould Belt. From this comprehensive dataset, combined with observations from the WISH and DIGIT surveys, we are able to conclude that:

- Water line profiles are dominated by emission from the actively shocked regions in outflows, the activity of which decreases in strength (i.e. has lower intensity and FWZI, see Fig. 8) and has fewer $J$-type shocks (i.e. fewer spot-shock components, see Fig. 15) as sources evolve from Class 0 to I. We also confirm the decrease in the force of the cooler and slower entrained outflowing gas, measured from low- $J \mathrm{CO}$, as young embedded protostars evolve.

- The ratio of mass in the entrained outflow to envelope mass (i.e. $M_{\text {out }} / M_{\text {env }}$ ) remains relatively constant between Class 0 and I with a median of approximately $1 \%$, consistent with a core-to-star formation efficiency of approximately $50 \%$ and an outflow duty cycle of approximately $5 \%$.

- $F_{\mathrm{CO}} / \dot{M}_{\mathrm{acc}}$ is relatively constant with $L_{\mathrm{bol}}, T_{\mathrm{bol}}$ and $M_{\mathrm{env}}$, suggesting that the entrainment efficiency is constant and independent of the power and evolution of the driving source of the flow. The constant value of $F_{\mathrm{CO}} / \dot{M}_{\text {acc }}$ implies a median velocity at the wind launching radius of $6.3 \mathrm{~km} \mathrm{~s}^{-1}$. This 
in turn suggests an entrainment efficiency of approximately $30-60 \%$ if the wind is launched around $1 \mathrm{AU}$, or close to $100 \%$ if it is launched at larger radii.

- $L[\mathrm{OI}]$ is strongly correlated with $L_{\mathrm{bol}}$ but not with $M_{\mathrm{env}}$, in contrast to low- $J \mathrm{CO}$, which is strongly correlated with $M_{\text {env }}$ and more weakly related to $L_{\text {bol }}$. This suggests that $[\mathrm{OI}]$ is more closely related to the current accretion activity while low- $J \mathrm{CO}$ traces the average activity over timescales of approximately $10^{2}-10^{4} \mathrm{yr} . \mathrm{H}_{2} \mathrm{O}$ is more strongly correlated with $L_{\mathrm{bol}}$ than $M_{\mathrm{env}}$, but with a smaller difference than for low- $J \mathrm{CO}$, consistent with it tracing actively shocked material between the wind and entrained outflow.

- L[OI] does not vary significantly between Class 0 and I, likely because the molecular to atomic ratio in the wind and jet decreases as the source evolves, as suggested by Nisini et al. (2015). This could be caused by increased temperature and decreased density (and thus shielding) in more evolved sources. [OI] is therefore a poor tracer of the timeaveraged mass-loss rate, and thus a poor alternative to $\mathrm{CO}$.

- Infall signatures are predominantly seen in Class 0 sources in both $\mathrm{H}_{2} \mathrm{O}$ and $\mathrm{HCO}^{+}$single-dish observations, but with little overlap in detections between the two tracers. However, infall signatures remain elusive in the majority of sources. Thus, while water is a good tracer of infall, it is by no means the universal tracer needed to understand how this proceeds in general.

- The conclusions drawn from the WISH sample hold and become more statistically robust when the combined WISH+WILL+DIGIT sample is analysed.

Further exploitation of these data and this sample can be found for the HIFI data in San José-García (2015) and will be presented for the PACS data in Karska et al. (in prep.). The use of PACS and HIFI in this way is cementing the unique legacy of Herschel on the energetics of star formation and the origin of water in the interstellar medium.

Acknowledgements. We thank the anonymous referee for constructive comments that improved the clarity and content of the paper. J.C.M. acknowledges support from grant 614.001.008 from The Netherlands Organisation for Scientific Research (NWO), from the European Union A-ERC grant 291141 CHEMPLAN, and from the European Research Council under the European Community's Horizon 2020 framework program (2014-2020) via the ERC Consolidator grant "From Cloud to Star Formation (CSF)" (project number 648505). Astrochemistry in Leiden is supported by The Netherlands Research School for Astronomy (NOVA), by a Spinoza grant, by a Royal Netherlands Academy of Arts and Sciences (KNAW) professor prize, and by the CHEMPLAN A-ERC grant. A.K. acknowledges support from the Foundation for Polish Science (FNP) and the Polish National Science Center grant 2013/11/N/ST9/00400. D.F. acknowledges support from the Italian Ministry of Education, Universities and Research project SIR (RBSI14ZRHR). HIFI has been designed and built by a consortium of institutes and university departments from across Europe, Canada and the United States under the leadership of SRON Netherlands Institute for Space Research, Groningen, The Netherlands and with major contributions from Germany, France and the US. Consortium members are: Canada: CSA, U.Waterloo France: CESR, LAB, LERMA, IRAM; Germany: KOSMA, MPIfR, MPS; Ireland, NUI Maynooth; Italy: ASI, IFSI-INAF, Osservatorio Astrofisico di ArcetriINAF; The Netherlands: SRON, TUD; Poland: CAMK, CBK; Spain: Observatorio Astronómico Nacional (IGN), Centro de Astrobiología (CSIC-INTA). Sweden: Chalmers University of Technology - MC2, RSS \& GARD; Onsala Space Observatory; Swedish National Space Board, Stockholm University Stockholm Observatory; Switzerland: ETH Zurich, FHNW; USA: Caltech, JPL, NHSC. PACS has been developed by a consortium of institutes led by MPE (Germany) and including UVIE (Austria); KU Leuven, CSL, IMEC (Belgium); CEA LAM (France); MPIA (Germany); INAF-IFSI/OAA/OAP/OAT, LENS, SISSA (Italy); IAC (Spain). This development has been supported by the funding agencies BMVIT (Austria), ESA-PRODEX (Belgium), CEA/CNES (France), DLR (Germany), ASI/INAF (Italy), and CICYT/MCYT (Spain). This research made use of ASTROPY, a community-developed core PYTHON package for Astronomy (Astropy Collaboration et al. 2013), and APLPY, an open-source package for plotting astronomical images with PYTHON hosted at http://aplpy.github . com.

\section{References}

Alcalá, J. M., Spezzi, L., Chapman, N., et al. 2008, ApJ, 676, 427 André, P., Ward-Thompson, D., \& Barsony, M. 1993, ApJ, 406, 122 André, P., Ward-Thompson, D., \& Barsony, M. 2000, in Protostars and Planets IV, eds. V. Mannings, A. Boss, \& S. Russell (Tucson: Univ. of Arizona Press), 59

André, P., Men'shchikov, A., Bontemps, S., et al. 2010, A\&A, 518, L102 Antoniucci, S., Nisini, B., Giannini, T., \& Lorenzetti, D. 2008, A\&A, 479, 503 Arce, H. G., \& Goodman, A. A. 2001, ApJ, 551, L171

Arce, H. G., Shepherd, D., Gueth, F., et al. 2007, in Protostars and Planets V, eds.

B. Reipurth, D. Jewitt, \& K. Keil (Tucson: Univ. of Arizona Press), 245

Arce, H. G., Mardones, D., Corder, S. A., et al. 2013, ApJ, 774, 39

Aso, Y., Ohashi, N., Saigo, K., et al. 2015, ApJ, 812, 27

Astropy Collaboration, Robitaille, T. P., Tollerud, E. J., et al. 2013, A\&A, 558, A33

Audard, M., Ábrahám, P., Dunham, M. M., et al. 2014, in Protostars and Planets VI, eds. H. Beuther, R. Klessen, C. Dullemond, \& T. Henning (Tucson: Univ. of Arizona Press), 387

Bachiller, R., Martin-Pintado, J., \& Planesas, P. 1991, A\&A, 251, 639

Bontemps, S., André, P., Terebey, S., \& Cabrit, S. 1996, A\&A, 311, 858

Brown, J. M., Pontoppidan, K. M., van Dishoeck, E. F., et al. 2013, ApJ, 770, 94

Buckle, J. V., Hills, R. E., Smith, H., et al. 2009, MNRAS, 399, 1026

Busquet, G., Lefloch, B., Benedettini, M., et al. 2014, A\&A, 561, A120

Cabrit, S. 2009, Observational Constraints to Steady Jet Models in Young Stars, eds. K. Tsinganos, T. Ray, \& M. Stute, 247

Cabrit, S., \& Bertout, C. 1990, ApJ, 348, 530

Cabrit, S., \& Bertout, C. 1992, A\&A, 261, 274

Caratti o Garatti, A., Garcia Lopez, R., Scholz, A., et al. 2011, A\&A, 526, L1

Caratti o Garatti, A., Garcia Lopez, R., Antoniucci, S., et al. 2012, A\&A, 538, A64

Carney, M. T., Yıldız, U. A., Mottram, J. C., et al. 2016, A\&A, 586, A44 Caselli, P., Benson, P. J., Myers, P. C., \& Tafalla, M. 2002, ApJ, 572, 238 Chabrier, G. 2005, in The Initial Mass Function 50 Years Later, eds. E. Corbelli,

F. Palla, \& H. Zinnecker, Astrophys. Space Sci. Lib., 327, 41

Chen, H., Myers, P. C., Ladd, E. F., \& Wood, D. O. S. 1995, ApJ, 445, 377 Codella, C., Cabrit, S., Gueth, F., et al. 2014, A\&A, 568, L5

Crapsi, A., van Dishoeck, E. F., Hogerheijde, M. R., Pontoppidan, K. M., \& Dullemond, C. P. 2008, A\&A, 486, 245

Curtis, E. I., Richer, J. S., \& Buckle, J. V. 2010, MNRAS, 401, 455

Daniel, F., Dubernet, M.-L., \& Grosjean, A. 2011, A\&A, 536, A76

Davis, C. J., Chrysostomou, A., Hatchell, J., et al. 2010, MNRAS, 405, 759

de Geus, E. J., de Zeeuw, P. T., \& Lub, J. 1989, A\&A, 216, 44

de Graauw, T., Helmich, F. P., Phillips, T. G., et al. 2010, A\&A, 518, L6

Di Francesco, J., Johnstone, D., Kirk, H., MacKenzie, T., \& Ledwosinska, E. 2008, ApJS, 175, 277

Dougados, C., Bacciotti, F., Cabrit, S., \& Nisini, B. 2010, in Lect. Notes Phys.

793, eds. P. J. V. Garcia, \& J. M. Ferreira (Berlin: Springer Verlag), 213 Downes, T. P., \& Cabrit, S. 2007, A\&A, 471, 873

Duarte-Cabral, A., Dobbs, C. L., Peretto, N., \& Fuller, G. A. 2011, A\&A, 528, A50

Duarte-Cabral, A., Bontemps, S., Motte, F., et al. 2013, A\&A, 558, A125 Dubernet, M.-L., Daniel, F., Grosjean, A., \& Lin, C. Y. 2009, A\&A, 497, 911

Dunham, M. M., Evans, II, N. J., Bourke, T. L., et al. 2006, ApJ, 651, 945

Dunham, M. M., Evans, II, N. J., Terebey, S., Dullemond, C. P., \& Young, C. H. 2010, ApJ, 710, 470

Dunham, M. M., Stutz, A. M., Allen, L. E., et al. 2014, in Protostars and Planets VI, eds. H. Beuther, R. Klessen, C. Dullemond, \& T. Henning (Tucson: Univ. of Arizona Press), 195

Dunham, M. M., Allen, L. E., Evans, II, N. J., et al. 2015, ApJS, 220, 11

Ellerbroek, L. E., Podio, L., Kaper, L., et al. 2013, A\&A, 551, A5

Enoch, M. L., Evans, II, N. J., Sargent, A. I., \& Glenn, J. 2009, ApJ, 692, 973

Evans, II, N. J., Allen, L. E., Blake, G. A., et al. 2003, PASP, 115, 965

Evans, II, N. J., Dunham, M. M., Jørgensen, J. K., et al. 2009, ApJS, 181, 321

Evans, II, N. J., Di Francesco, J., Lee, J.-E., et al. 2015, ApJ, 814, 22

Fedele, D., Bruderer, S., van Dishoeck, E. F., et al. 2013, A\&A, 559, A77

Ferreira, J., Dougados, C., \& Cabrit, S. 2006, A\&A, 453, 785

Fischer, C. F., \& Saha, H. P. 1983, Phys. Rev. A, 28, 3169

Fischer, W. J., Megeath, S. T., Tobin, J. J., et al. 2012, ApJ, 756, 99

Frank, A., Ray, T. P., Cabrit, S., et al. 2014, in Protostars and Planets VI, eds.

H. Beuther, R. Klessen, C. Dullemond, \& T. Henning (Tucson: Univ. of Arizona Press), 451

Frerking, M. A., Langer, W. D., \& Wilson, R. W. 1982, ApJ, 262, 590 
Furlan, E., Fischer, W. J., Ali, B., et al. 2016, ApJS, 224, 5

Gallagher, D. B., Irace, W. R., \& Werner, M. W. 2003, in IR Space Telescopes and Instruments, ed. J. C. Mather, Proc. SPIE, 4850, 17

Giannini, T., Nisini, B., \& Lorenzetti, D. 2001, ApJ, 555, 40

Gould, B. G. 1879, Resultados del Observatorio Nacional Argentino, 1, 0

Green, J. D., Evans, II, N. J., Jørgensen, J. K., et al. 2013a, ApJ, 770, 123

Green, J. D., Evans, II, N. J., Kóspál, Á., et al. 2013b, ApJ, 772, 117

Green, J. D., Yang, Y.-L., Evans, II, N. J., et al. 2016, AJ, 151, 75

Greene, T. P., Wilking, B. A., André, P., Young, E. T., \& Lada, C. J. 1994, ApJ, 434,614

Gregersen, E. M., Evans, II, N. J., Zhou, S., \& Choi, M. 1997, ApJ, 484, 256

Griffin, M. J., Abergel, A., Abreu, A., et al. 2010, A\&A, 518, L3

Harsono, D., Jørgensen, J. K., van Dishoeck, E. F., et al. 2014, A\&A, 562, A77

Hatchell, J., Terebey, S., Huard, T., et al. 2012, ApJ, 754, 104

Heiderman, A., \& Evans, II, N. J. 2015, ApJ, 806, 231

Heiderman, A., Evans, II, N. J., Allen, L. E., Huard, T., \& Heyer, M. 2010, ApJ, 723, 1019

Herczeg, G. J., Karska, A., Bruderer, S., et al. 2012, A\&A, 540, A84

Hirota, T., Bushimata, T., Choi, Y. K., et al. 2008, PASJ, 60, 37

Hogerheijde, M. R., Jansen, D. J., \& van Dishoeck, E. F. 1995, A\&A, 294, 792

Hollenbach, D. 1985, Icarus, 61, 36

Howard, C. D., Sandell, G., Vacca, W. D., et al. 2013, ApJ, 776, 21

Ingleby, L., Calvet, N., Herczeg, G., et al. 2013, ApJ, 767, 112

Jansen, D. J., van Dishoeck, E. F., Keene, J., Boreiko, R. T., \& Betz, A. L. 1996, A\&A, 309, 899

Jenness, T., Currie, M. J., Tilanus, R. P. J., et al. 2015, MNRAS, 453, 73

Johnstone, D., Hendricks, B., Herczeg, G. J., \& Bruderer, S. 2013, ApJ, 765, 133

Jørgensen, J. K., Johnstone, D., Kirk, H., \& Myers, P. C. 2007, ApJ, 656, 293

Jørgensen, J. K., Johnstone, D., Kirk, H., et al. 2008, ApJ, 683, 822

Jørgensen, J. K., van Dishoeck, E. F., Visser, R., et al. 2009, A\&A, 507, 861

Jørgensen, J. K., Visser, R., Sakai, N., et al. 2013, ApJ, 779, L22

Jørgensen, J. K., Visser, R., Williams, J. P., \& Bergin, E. A. 2015, A\&A, 579, A23

Karska, A., Herczeg, G. J., van Dishoeck, E. F., et al. 2013, A\&A, 552, A141

Karska, A., Herpin, F., Bruderer, S., et al. 2014a, A\&A, 562, A45

Karska, A., Kristensen, L. E., van Dishoeck, E. F., et al. 2014b, A\&A, 572, A9

Kauffmann, J., Bertoldi, F., Bourke, T. L., Evans, II, N. J., \& Lee, C. W. 2008 A\&A, 487, 993

Kenyon, S. J., Gómez, M., \& Whitney, B. A. 2008, in Low Mass Star Formation in the Taurus-Auriga Clouds, ed. B. Reipurth, 405

Kim, H. J., Evans, II, N. J., Dunham, M. M., Lee, J.-E., \& Pontoppidan, K. M 2012, ApJ, 758, 38

Kirk, H., Myers, P. C., Bourke, T. L., et al. 2013, ApJ, 766, 115

Klaassen, P. D., Juhasz, A., Mathews, G. S., et al. 2013, A\&A, 555, A73

Knude, J., \& Høg, E. 1998, A\&A, 338, 897

Konigl, A., \& Pudritz, R. E. 2000, in Protostars and Planets IV, eds. V. Mannings, A. Boss, \& S. Russell (Tucson: Univ. of Arizona Press), 759

Könyves, V., André, P., Men'shchikov, A., et al. 2015, A\&A, 584, A91

Kristensen, L. E., van Dishoeck, E. F., Tafalla, M., et al. 2011, A\&A, 531, L1

Kristensen, L. E., van Dishoeck, E. F., Bergin, E. A., et al. 2012, A\&A, 542, A8

Kristensen, L. E., van Dishoeck, E. F., Benz, A. O., et al. 2013, A\&A, 557, A23

Kristensen, L. E., van Dishoeck, E. F., Mottram, J. C., et al. 2017, A\&A

Kwon, W., Looney, L. W., Crutcher, R. M., \& Kirk, J. M. 2006, ApJ, 653, 1358

Lada, C. J. 1987, in Star Forming Regions, eds. M. Peimbert, \& J. Jugaku, IAU Symp., 115, 1

Lada, C. J., \& Wilking, B. A. 1984, ApJ, 287, 610

Lee, C.-F., Hasegawa, T. I., Hirano, N., et al. 2010a, ApJ, 713, 731

Lee, J.-E., Lee, H.-G., Shinn, J.-H., et al. 2010b, ApJ, 709, L74

Lee, C.-F., Hirano, N., Zhang, Q., et al. 2015, ApJ, 805, 186

Lindberg, J. E., Jørgensen, J. K., Green, J. D., et al. 2014, A\&A, 565, A29

Loinard, L. 2013, in Advancing the Physics of Cosmic Distances, ed. R. de Grijs, IAU Symp., 289, 36

Manoj, P., Watson, D. M., Neufeld, D. A., et al. 2013, ApJ, 763, 83

Mardones, D., Myers, P. C., Tafalla, M., et al. 1997, ApJ, 489, 719

Marseille, M. G., van der Tak, F. F. S., Herpin, F., \& Jacq, T. 2010, A\&A, 522, A40

Matuszak, M., Karska, A., Kristensen, L. E., et al. 2015, A\&A, 578, A20

Maud, L. T., Moore, T. J. T., Lumsden, S. L., et al. 2015, MNRAS, 453, 645

Maury, A. J., André, P., Men'shchikov, A., Könyves, V., \& Bontemps, S. 2011, A\&A, 535, A77

Megeath, S. T., Gutermuth, R., Muzerolle, J., et al. 2012, AJ, 144, 192

Mottram, J. C., van Dishoeck, E. F., Schmalzl, M., et al. 2013, A\&A, 558, A126

Mottram, J. C., Kristensen, L. E., van Dishoeck, E. F., et al. 2014, A\&A, 572, A21

Müller, H. S. P., Thorwirth, S., Roth, D. A., \& Winnewisser, G. 2001, A\&A, 370, L49
Müller, H. S. P., Schlöder, F., Stutzki, J., \& Winnewisser, G. 2005, J. Mol. Struct., 742,215

Murillo, N. M., Lai, S.-P., Bruderer, S., Harsono, D., \& van Dishoeck, E. F. 2013, A\&A, 560, A103

Myers, P. C. 2008, ApJ, 687, 340

Myers, P. C., \& Ladd, E. F. 1993, ApJ, 413, L47

Myers, P. C., Evans, II, N. J., \& Ohashi, N. 2000, in Protostars and Planets IV, eds. V. Mannings, A. Boss, \& S. Russell (Tucson: Univ. of Arizona Press), 217

Nagy, Z., Van der Tak, F. F. S., Ossenkopf, V., et al. 2013, A\&A, 550, A96

Nisini, B., Giannini, T., \& Lorenzetti, D. 2002, ApJ, 574, 246

Nisini, B., Antoniucci, S., Giannini, T., \& Lorenzetti, D. 2005, A\&A, 429, 543

Nisini, B., Benedettini, M., Codella, C., et al. 2010, A\&A, 518, L120

Nisini, B., Santangelo, G., Giannini, T., et al. 2015, ApJ, 801, 121

Offner, S. S. R., Clark, P. C., Hennebelle, P., et al. 2014, in Protostars and Planets

VI, eds. H. Beuther, R. Klessen, C. Dullemond, \& T. Henning (Tucson: Univ. of Arizona Press), 53

Ortiz-León, G. N., Dzib, S. A., Kounkel, M. A., et al. 2017, ApJ, 834, 143

Ott, S. 2010, in Astronomical Data Analysis Software and Systems XIX, eds. Y. Mizumoto, K.-I. Morita, \& M. Ohishi, ASP Conf. Ser., 434, 139

Padgett, D. L., Rebull, L. M., Stapelfeldt, K. R., et al. 2008, ApJ, 672, 1013

Panoglou, D., Cabrit, S., Pineau Des Forêts, G., et al. 2012, A\&A, 538, A2

Peterson, D. E., Caratti o Garatti, A., Bourke, T. L., et al. 2011, ApJS, 194, 43

Pickett, H. M., Poynter, I. R. L., Cohen, E. A., et al. 2010, J. Quant. Spectr. Rad. Transf., 111, 1617

Pilbratt, G. L., Riedinger, J. R., Passvogel, T., et al. 2010, A\&A, 518, L1

Plunkett, A. L., Arce, H. G., Corder, S. A., et al. 2013, ApJ, 774, 22

Podio, L., Kamp, I., Flower, D., et al. 2012, A\&A, 545, A44

Podio, L., Kamp, I., Codella, C., et al. 2013, ApJ, 766, L5

Poglitsch, A., Waelkens, C., Geis, N., et al. 2010, A\&A, 518, L2

Rebull, L. M., Stapelfeldt, K. R., Evans, II, N. J., et al. 2007, ApJS, 171, 447

Rebull, L. M., Padgett, D. L., McCabe, C.-E., et al. 2010, ApJS, 186, 259

Roelfsema, P. R., Helmich, F. P., Teyssier, D., et al. 2012, A\&A, 537, A17

Sadavoy, S. I., Di Francesco, J., \& Johnstone, D. 2010, ApJ, 718, L32

Safron, E. J., Fischer, W. J., Megeath, S. T., et al. 2015, ApJ, 800, L5

San José-García, I. 2015, Ph.D. Thesis, Leiden University, The Netherlands

San José-García, I., Mottram, J. C., Kristensen, L. E., et al. 2013, A\&A, 553, A125

San José-García, I., Mottram, J. C., van Dishoeck, E. F., et al. 2016, A\&A, 585, A103

Santangelo, G., Nisini, B., Antoniucci, S., et al. 2013, A\&A, 557, A22

Santangelo, G., Nisini, B., Codella, C., et al. 2014, A\&A, 568, A125

Santiago-García, J., Tafalla, M., Johnstone, D., \& Bachiller, R. 2009, A\&A, 495, 169

Schmalzl, M., Visser, R., Walsh, C., et al. 2014, A\&A, 572, A81

Shirley, Y. L. 2015, PASP, 127, 299

Shu, F. H., Adams, F. C., \& Lizano, S. 1987, ARA\&A, 25, 23

Skrutskie, M. F., Cutri, R. M., Stiening, R., et al. 2006, AJ, 131, 1163

Swinyard, B. M., Burgdorf, M. J., Clegg, P. E., et al. 1998, in Infrared Astronomical Instrumentation, ed. A. M. Fowler, Proc. SPIE, 3354, 888

Tafalla, M., Santiago-García, J., Hacar, A., \& Bachiller, R. 2010, A\&A, 522, A91

Tafalla, M., Liseau, R., Nisini, B., et al. 2013, A\&A, 551, A116

Tobin, J. J., Looney, L. W., Mundy, L. G., Kwon, W., \& Hamidouche, M. 2007, ApJ, 659, 1404

Tobin, J. J., Hartmann, L., Chiang, H.-F., et al. 2012, Nature, 492, 83

Tobin, J. J., Looney, L. W., Wilner, D. J., et al. 2015, ApJ, 805, 125

Tobin, J. J., Looney, L. W., Li, Z.-Y., et al. 2016, ApJ, 818, 73

van der Marel, N., Kristensen, L. E., Visser, R., et al. 2013, A\&A, 556, A76

van Dishoeck, E. F., Kristensen, L. E., Benz, A. O., et al. 2011, PASP, 123, 138

van Kempen, T. A., van Dishoeck, E. F., Salter, D. M., et al. 2009, A\&A, 498, 167

Vassilev, V., Meledin, D., Lapkin, I., et al. 2008, A\&A, 490, 1157

Visser, R., Bergin, E. A., \& Jørgensen, J. K. 2015, A\&A, 577, A102

Watson, D. M., Calvet, N. P., Fischer, W. J., et al. 2016, ApJ, 828, 52

White, G. J., Drabek-Maunder, E., Rosolowsky, E., et al. 2015, MNRAS, 447, 1996

Whittet, D. C. B., Prusti, T., Franco, G. A. P., et al. 1997, A\&A, 327, 1194

Yen, H.-W., Takakuwa, S., Ohashi, N., et al. 2014, ApJ, 793, 1

Yildı, U. A., Kristensen, L. E., van Dishoeck, E. F., et al. 2013, A\&A, 556, A89

Yıldız, U. A., Kristensen, L. E., van Dishoeck, E. F., et al. 2015, A\&A, 576, A109

Yvart, W., Cabrit, S., Pineau des Forêts, G., \& Ferreira, J. 2016, A\&A, 585, A74

Zink, L. R., Evenson, K. M., Matsushima, F., Nelis, T., \& Robinson, R. L. 1991, ApJ, 371, L85 
1 Leiden Observatory, Leiden University, PO Box 9513, 2300 RA Leiden, The Netherlands

2 Max Planck Institute for Astronomy, Königstuhl 17, 69117 Heidelberg, Germany

${ }^{3}$ Max Planck Institut für Extraterrestrische Physik, Giessenbachstrasse 1, 85748 Garching, Germany

${ }^{4}$ Harvard-Smithsonian Center for Astrophysics, 60 Garden Street, Cambridge, MA 02138, USA

5 Centre for Star and Planet Formation, Niels Bohr Institute and Natural History Museum of Denmark, University of Copenhagen, Øster Voldgade 5-7, 1350 Copenhagen K, Denmark

${ }^{6}$ Centre for Astronomy, Nicolaus Copernicus University, Faculty of Physics, Astronomy and Informatics, Grudziadzka 5, 87100 Torun, Poland

7 Kavli Institut for Astronomy and Astrophysics, Yi He Yuan Lu 5, HaiDian Qu, Peking University, 100871 Beijing, PR China

${ }^{8}$ Laboratoire AIM, CEA/DSM-CNRS-Université Paris Diderot, IRFU/Service d'Astrophysique, CEA Saclay, 91191 Gif-sur-Yvette, France

9 Univ. Bordeaux, LAB, UMR5804, 33270 Floirac, France

10 CNRS, LAB, UMR5804, 33270 Floirac, France

11 LERMA, Observatoire de Paris, UMR 8112 du CNRS, ENS, UPMC, UCP, 61 Av. de l'Observatoire, 75014 Paris, France
12 Institut de Planétologie et d'Astrophysique de Grenoble (IPAG) UMR 5274, 38041 Grenoble, France

13 Department of Astronomy, University of Texas at Austin, Austin, TX 78712, USA

14 INAF-Osservatorio Astrofisico di Arcetri, L.go E. Fermi 5, 50125 Firenze, Italy

15 Space Telescope Science Institute, 3700 Baltimore, MD, USA

16 Universität Heidelberg, Zentrum für Astronomie, Institut für Theoretische Astrophysik (ITA), Albert-Ueberle-Str. 2, 69120 Heidelberg, Germany

17 NRC Herzberg Astronomy and Astrophysics, 5071 West Saanich Rd, Victoria, BC, V9E 2E7, Canada

18 Department of Physics and Astronomy, University of Victoria, Victoria, BC, V8P 1A1, Canada

19 INAF-Osservatorio Astronomico di Roma, via Frascati 33, 00040 Monte Porzio Catone, Italy

20 Observatorio Astronomico Nacional (OAN-IGN), Alfonso XII 3, 28014 Madrid, Spain

${ }^{21}$ European Southern Observatory, Karl-Schwarzschild-Strasse 2, 85748 Garching, Germany

22 Jet Propulsion Laboratory, California Institute of Technology, 4800 Oak Grove Drive, Pasadena CA, 91109, USA 


\section{Appendix A: Property determination}

This section presents the various properties of the WILL sources, and details of how they were determined.

\section{A.1. Spectral energy distributions}

In constructing the spectral energy distributions (SEDs) of the WILL sources, the photometric flux densities from the near-IR to $24 \mu \mathrm{m}$ from 2MASS (Skrutskie et al. 2006) and Spitzer have been collected, where detected, from the latest determinations by Dunham et al. (2015). Flux densities for detections at 450 and/or $850 \mu \mathrm{m}$ with SCUBA on the JCMT were taken from the catalogue of Di Francesco et al. (2008), and the $1.3 \mathrm{~mm}$ detections with MAMBO on the IRAM 30m telescope by Maury et al. (2011) for Aquila, Serpens South and W40 were also included.

All WILL sources lie within the Herschel PACS and SPIRE photometric maps observed in parallel-mode at 70, 160, 250, 350 and $500 \mu \mathrm{m}$ as part of the Herschel Gould Belt Survey, with many also within the smaller regions observed in PACS-only mode at 100 and $160 \mu \mathrm{m}$. Where both parallel and PACS-only mode observations are available at $160 \mu \mathrm{m}$ we use the data taken in PACS-only mode, as these were observed with a slower scanning speed and thus are of better quality than those data taken in parallel with SPIRE observations. Processed and calibrated mosaics were downloaded from the Herschel Science Archive (HSA ${ }^{7}$, see also the Herschel Gould Belt survey archive for further products ${ }^{8}$ ). The observation identification numbers for these maps are given in Table B.2.

Aperture photometry was then performed using PYTHON routines from the ASTROPY package to extract flux densities for all sources at all available wavelengths. The starting value for the source aperture, inner and outer sky radii for each source at each wavelength was set at 6,10 and 12 pixels, typically corresponding to 6,10 and 12 times the beam size. Rings at these radii were then overlaid on images of the data and the radii adjusted to best encompass each source and local background respectively, while excluding nearby sources. Aperture correction factors were calculated and applied for each combination of aperture and sky annuli using the relevant PACS and SPIRE point source function images.

In some cases, additional nearby sources are blended with the primary WILL source at longer wavelengths where the Herschel beam becomes large. For these sources, we specify the longest wavelength where the sources are reliably separated. The flux densities at longer wavelengths are then scaled down by the ratio of the flux densities in the reliable image between the aperture used for that wavelength and the aperture used for the longer wavelength data. For example, if a source is blended at $500 \mu \mathrm{m}$, then the flux density at that wavelength is scaled down by the ratio of the flux density at $350 \mu \mathrm{m}$ to the flux density in the $350 \mu \mathrm{m}$ image within the region defined by the aperture used for the $500 \mu \mathrm{m}$ data.

Finally, continuum flux densities from the WILL PACS spectra were also obtained in the same manner as for the WISH sources (see Karska et al. 2013, for details). However, it is sometimes difficult in the PACS spectral maps to separate the emission related to the protostar from surrounding emission. Therefore, in cases where the PACS spectral continuum is significantly higher than obtained from the broad-band photometric maps, the latter is preferred and the PACS spectral continuum flux densities

\footnotetext{
7 http://www.cosmos.esa.int/web/herschel/

science-archive/

8 http://gouldbelt-herschel.cea.fr/archives
}

are not included in the SED. The broad-band photometric flux densities for all sources are given in Table A.1, with the PACS spectral continuum flux densities given separately in Table A.2. The SEDs of all sources are shown in Fig. A.1.

For Aquila sources 01-04, the peak PACS continuum is offset from the coordinates in Maury et al. (2011), and so extraction of the SED and ground-based molecular line emission is performed at the peak flux position.

\section{A.2. Continuum properties}

A number of properties are often calculated from YSO SEDs in order to characterise them. While previous determinations have been made for the better-studied members of the WILL sample, variations in method and implementation could lead to biases or systematic effects between sources in different regions. In addition, Herschel data provide significantly higher-resolution images for flux density determination than were previously available between 70 and $250 \mu \mathrm{m}$ (e.g. Herschel has a FWHM of $\sim 8^{\prime \prime}$ at $100 \mu \mathrm{m}$ compared to $3^{\prime} \times 5^{\prime}$ for IRAS). In order to be consistent with other Herschel surveys, most notably the WISH and DIGIT surveys, we follow the definitions in Dunham et al. (2010) and calculate the bolometric luminosity $\left(L_{\text {bol }}\right)$ using:

$L_{\mathrm{bol}}=4 \pi \mathrm{d}^{2} \int_{0}^{\infty} F_{\nu} \mathrm{d} v$

the sub-mm luminosity using:

$L_{\mathrm{smm}}=4 \pi \mathrm{d}^{2} \int_{0}^{v=c / 350 \mu \mathrm{m}} F_{\nu} \mathrm{d} v$,

and bolometric temperature using:

$T_{\text {bol }}=1.25 \times 10^{-11} \frac{\int_{0}^{\infty} v F_{\nu} \mathrm{d} v}{\int_{0}^{\infty} F_{v} \mathrm{~d} v}$.

In these equations, $F_{v}$ is the flux density at frequency $v$, and $d$ is the distance to the source. The integrals were calculated over the available SED flux densities using trapezium integration, which was found by Karska et al. (2013) to provide the most consistent results for the WISH survey sources.

The infrared spectral index (see Lada 1987) is also calculated for those sources where the source is detected in at least three wavelengths between 2 and $25 \mu \mathrm{m}$ using a least-squares linear fit to the logarithms of wavelength and flux density such that:

$\alpha_{\mathrm{IR}}=\frac{\mathrm{d} \log _{10}\left(\lambda F_{\lambda}\right)}{\mathrm{d} \log _{10}(\lambda)}$,

where $F_{\lambda}$ is the flux density at wavelength $\lambda$.

Finally, for the sources in the Aquila Rift we take the envelope mass $\left(M_{\text {env }}\right)$ from Maury et al. (2011), calculated from the integrated intensity at $1.2 \mathrm{~mm}$, corrected to the updated distance for that region. In other regions we calculate $M_{\text {env }}$ for those sources with SCUBA $850 \mu \mathrm{m}$ observations using Eq. (1) from Jørgensen et al. (2009):

$M_{\mathrm{env}}=0.44\left(\frac{L_{\mathrm{bol}}}{1 L_{\odot}}\right)^{-0.36}\left(\frac{S_{15^{\prime \prime}, 850 \mu \mathrm{m}}}{1 \mathrm{Jy} \mathrm{beam}^{-1}}\right)^{1.2}\left(\frac{\mathrm{d}}{125 \mathrm{pc}}\right)^{1.2} M_{\odot}$,

where $S_{15^{\prime \prime}, 850 \mu \mathrm{m}}$ is the peak SCUBA $850 \mu \mathrm{m}$ flux density in a $15^{\prime \prime}$ pixel. This empirical relation was derived from comparison of observed source properties with the results of dust radiative transfer models. The results of these calculations are summarised in Table 1. 
J. C. Mottram et al.: Outflows, infall and evolution of a sample of embedded low-mass protostars

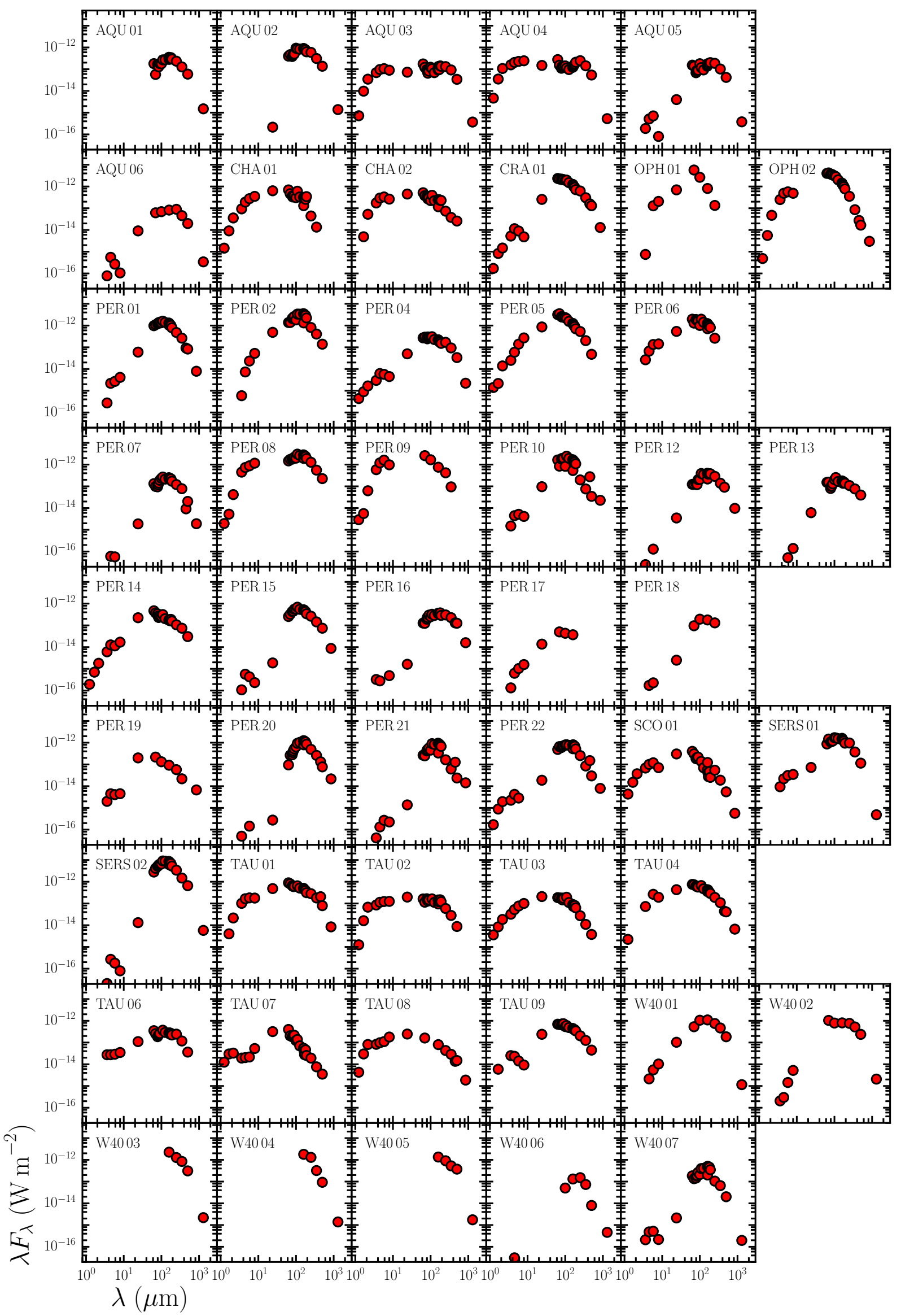

Fig. A.1. SEDs for all WILL sources. Data points from the PACS spectra are only included when used in the $L_{\text {bol }}$ calculations. 
Table A.1. SED photometric continuum fluxes.

\begin{tabular}{|c|c|c|c|c|c|c|c|c|c|c|c|c|c|}
\hline \multirow{3}{*}{$\begin{array}{l}\text { Name } \\
\text { AQU } 01 \\
\text { AQU } 02\end{array}$} & \multirow{3}{*}{$\begin{array}{c}\begin{array}{c}J \\
(\mathrm{mJy})\end{array} \\
- \\
-\end{array}$} & \multirow{3}{*}{$\begin{array}{c}\begin{array}{c}H \\
(\mathrm{mJy})\end{array} \\
- \\
-\end{array}$} & \multirow{3}{*}{$\begin{array}{c}\begin{array}{c}K_{\mathrm{S}} \\
(\mathrm{mJy})\end{array} \\
- \\
-\end{array}$} & \multicolumn{2}{|c|}{$\begin{array}{c}3.6 \\
(\mathrm{mJy})\end{array}$} & \multicolumn{2}{|c|}{$\begin{array}{c}4.5 \\
(\mathrm{mJy})\end{array}$} & \multicolumn{2}{|c|}{$\begin{array}{c}5.8 \\
(\mathrm{mJy})\end{array}$} & \multicolumn{2}{|c|}{$\begin{array}{c}8.0 \\
(\mathrm{mJy})\end{array}$} & \multicolumn{2}{|c|}{$\begin{array}{c}24 \\
(\mathrm{mJy})\end{array}$} \\
\hline & & & & - & & - & & - & & - & & - & \\
\hline & & & & - & & - & & - & & - & & $1.72 \pm$ & 0.60 \\
\hline AQU 03 & $0.30 \pm 0.06$ & $5.39 \pm 0.17$ & $25.00 \pm 0.60$ & $83.40 \pm$ & 4.17 & $145.00 \pm$ & 6.99 & $205.00 \pm$ & 9.76 & $239.00 \pm$ & 11.40 & $581.00 \pm$ & 54.10 \\
\hline AQU 04 & $92 \pm 0.09$ & $19.50 \pm 0.49$ & $.40 \pm 1.64$ & $190.00 \pm$ & 9.28 & $306.00 \pm$ & 15.90 & $441.00 \pm$ & 20.80 & $650.00 \pm$ & 31.20 & $1190.00 \pm$ & 118.00 \\
\hline AQU 05 & - & - & - & $0.22 \pm$ & 0.02 & $0.77 \pm$ & 0.08 & $1.37 \pm$ & 0.09 & $0.21 \pm$ & 0.10 & $31.90 \pm$ & 2.98 \\
\hline AQU 06 & - & - & - & $0.09 \pm$ & 0.01 & $0.83 \pm$ & 0.04 & $0.52 \pm$ & 0.06 & $0.28 \pm$ & 0.13 & $72.60 \pm$ & 6.85 \\
\hline CHA 01 & $60 \pm 0.06$ & $5.04 \pm 0.18$ & $5.70 \pm 0.61$ & $112.00 \pm$ & 10.10 & $287.00 \pm$ & 24.00 & $533.00 \pm$ & 27.00 & $939.00 \pm$ & 48.30 & $5080.00 \pm$ & 473.00 \\
\hline CHA 02 & - & $2.70 \pm 0.12$ & $3.00 \pm 1.10$ & $210.00 \pm$ & 10.00 & $440.00 \pm$ & 10.00 & $640.00 \pm$ & 31.00 & $700.00 \pm$ & 34.00 & $3600.00 \pm$ & 340.00 \\
\hline CRA 01 & $0.07 \pm 0.01$ & $0.46 \pm 0.05$ & $1.06 \pm 0.11$ & $6.30 \pm$ & & $17.20 \pm$ & 1.20 & $17.30 \pm$ & 1.10 & $12.90 \pm$ & 0.70 & $2040.00 \pm$ & 193.00 \\
\hline & - & - & - & $0.90 \pm$ & 0.25 & - & & $250.00 \pm$ & 30.00 & $550.00 \pm$ & 130.00 & $5600.00 \pm$ & 840.00 \\
\hline OPH 02 & $0.20 \pm 0.04$ & $3.10 \pm 0.28$ & $34.00 \pm 2.10$ & $300.00 \pm$ & 24.00 & $730.00 \pm$ & 48.00 & $1100.00 \pm$ & 55.00 & $1300.00 \pm$ & 65.00 & - & \\
\hline PER 01 & - & - & - & $0.33 \pm$ & & $3.30 \pm$ & 0.29 & $5.20 \pm$ & 0.36 & $11.00 \pm$ & 0.57 & $480.00 \pm$ & 51.00 \\
\hline & - & - & - & $71 \pm$ & & $11.00 \pm$ & 0.74 & $45.00 \pm$ & & $140.00 \pm$ & 7.10 & $3900.00 \pm$ & 420.00 \\
\hline $\mathrm{PL}$ & $0.18 \pm 0.01$ & $0.50 \pm 0.03$ & $1.20 \pm 0.08$ & $3.60 \pm$ & 0.2 & $9.30 \pm$ & 0.65 & $11.00 \pm$ & 0.77 & $12.00 \pm$ & 0.82 & $400.00 \pm$ & 42.00 \\
\hline & $8 \pm 0.03$ & $20 \pm 0.15$ & $10.00 \pm 0$ & $30.00 \pm$ & 2.3 & $89.00 \pm$ & 5.70 & $270.00 \pm$ & 13.00 & $720.00 \pm$ & 41.00 & $6900.00 \pm$ & 1200.00 \\
\hline & - & - & - & $32.00 \pm$ & 2.20 & $100.00 \pm$ & 6.00 & $260.00 \pm$ & 13.00 & $380.00 \pm$ & 23.00 & $4300.00 \pm$ & 460.00 \\
\hline PER 0 & - & - & - & - & & $0.09 \pm$ & 0.01 & $0.11 \pm$ & 0.03 & - & & $15.00 \pm$ & 1.60 \\
\hline & $30 \pm 0.04$ & $2.90 \pm 0$. & $.00 \pm 1.80$ & $540.00 \pm$ & & $1100.00 \pm$ & 82.00 & $1700.00 \pm 2$ & 210.00 & $3100.00 \pm$ & 190.00 & - & \\
\hline PER 09 & $0 \pm 0.06$ & $0 \pm 0$ & $.00 \pm 2.80$ & $700.00 \pm$ & 76.00 & $1800.00 \pm$ & 170.00 & $3100.00 \pm 2$ & 280.00 & $2600.00 \pm$ & 250.00 & - & \\
\hline PER 10 & - & - & - & $1.80 \pm$ & 0.18 & $6.70 \pm$ & 0.66 & $10.00 \pm$ & 0.83 & $11.00 \pm$ & 0.78 & $770.00 \pm$ & 82.00 \\
\hline & - & - & - & $0.03 \pm$ & 0.01 & - & & & & - & & & \\
\hline $\mathrm{PL}$ & - & - & - & - & & $0.02 \pm$ & 0.01 & $0.10 \pm$ & 0. & $0.37 \pm$ & 0.04 & $0 \pm$ & 5.20 \\
\hline & $08 \pm 0.01$ & $0.39 \pm 0.02$ & $1.30 \pm 0.10$ & $7.30 \pm$ & 0.5 & & & & & & & $1800.00 \pm$ & 190.00 \\
\hline & - & - & - & $0.13 \pm$ & & & & $0.83 \pm$ & 0.07 & & 0.0 & & 1.50 \\
\hline PER 1 & - & - & - & $0.39 \pm$ & 0.0 & $0.42 \pm$ & 0.14 & - & & $1.30 \pm$ & 0.11 & $13.00 \pm$ & 1.40 \\
\hline & - & - & - & $0.16 \pm$ & 0.01 & $0.94 \pm$ & & $2.00 \pm$ & & $4.20 \pm$ & 0.30 & $110.00 \pm$ & 12.00 \\
\hline & - & - & - & - & & $0.26 \pm$ & 0.0 & $0.44 \pm$ & 0.05 & - & & $20.00 \pm$ & 2.10 \\
\hline PER 1 & - & - & - & $2.40 \pm$ & 0.17 & $6.70 \pm$ & 0.34 & $7.90 \pm$ & 0.40 & $12.00 \pm$ & 0.60 & $1600.00 \pm$ & 170.00 \\
\hline & - & - & - & & & - & & & & - & & & 0.34 \\
\hline & - & - & - & & & $0.20 \pm$ & 0.06 & & 0.0 & $0.60 \pm$ & 0.12 & $0 \pm$ & 1.20 \\
\hline & $7+0$ & $9 \pm 0$ & $0 \pm 0$ & & & & & & & - & & $0 \pm$ & 20.00 \\
\hline & $8 \pm 0$ & $3 \pm 0$ & $30 \pm$ & \pm & & & & $231.00 \pm$ & 10.9 & $189.00 \pm$ & 11.60 & $2430.00 \pm$ & 248.00 \\
\hline SERS & - & - & - & $11.30 \pm$ & & $32.80 \pm$ & 1.62 & $60.20 \pm$ & 2.8 & $92.60 \pm$ & 4.56 & $582.00 \pm$ & 105.00 \\
\hline & - & - & - & & & $0.40 \pm$ & & & & $0.21 \pm$ & & & 11.90 \\
\hline & - & $22 \pm 0.12$ & $.51 \pm 0.31$ & & & $248.06 \pm$ & 11. & & 16. & $473.22 \pm$ & 21.79 & $3887.74 \pm$ & 143.23 \\
\hline & $0.52 \pm 0.04$ & $4+0$ & $.70 \pm 1 . C$ & & & $170.04 \pm$ & & $238.07 \pm$ & 10.96 & $333.47 \pm$ & 15.36 & $1576.52 \pm$ & 58.08 \\
\hline & & $88 \pm 0.2$ & $27 \pm 0$. & & & $78.44 \pm$ & 3.61 & & & & & & 61.38 \\
\hline & $2 \pm 0.06$ & - & - & & 4. & - & & $511.33 \pm$ & 23.55 & $514.12 \pm$ & 23.68 & $3450.95 \pm$ & 128.24 \\
\hline & - & - & - & & & $41.17 \pm$ & 1.9 & & & & 4.2 & & 32.51 \\
\hline & $4+01$ & $0+0$ & $44+0$ & & & & & & & & 6.0 & & 93.76 \\
\hline TAU 0 & $77 \pm 0.07$ & $16.50 \pm 0.5$ & $.55 \pm 1.19$ & $99.21 \pm$ & 4.5 & & & & & $477.60 \pm$ & 21.99 & $1966.52 \pm$ & 72.44 \\
\hline & - & $3.27 \pm 0.1$ & - & $29.96 \pm$ & 1.38 & & 1.6 & & & & 1.13 & $1912.93 \pm$ & 70.47 \\
\hline & - & - & - & - & & & & & & & 3.7 & $824.00 \pm$ & 78.40 \\
\hline & - & - & - & $0.25 \pm$ & 0.09 & $0.45 \pm$ & 0.07 & $2.80 \pm$ & 1.01 & $13.90 \pm$ & 3.94 & - & \\
\hline & - & - & - & - & & - & & - & & - & & - & \\
\hline & - & - & - & - & & - & & - & & - & & - & \\
\hline & - & - & - & - & & - & & - & & - & & - & \\
\hline & - & - & - & - & & $0.05 \pm$ & & - & & - & & - & \\
\hline W40 07 & - & - & - & $0.25 \pm$ & 0.03 & $0.73 \pm$ & 0.05 & $1.00 \pm$ & 0.07 & $0.58 \pm$ & 0.06 & $17.00 \pm$ & 1.58 \\
\hline
\end{tabular}

Notes. All numerical wavelengths in $\mu \mathrm{m} .{ }^{(a)}$ A $20 \%$ uncertainty is assumed for the SCUBA fluxes. ${ }^{(b)}$ Wavelength used to correct for contamination of nearby sources in longer-wavelength Herschel fluxes. ${ }^{(c)}$ Flux corrected for contamination of nearby sources using lower-wavelength Herschel map.

\section{A.3. PACS line properties and detection statistics}

Table 3 gives the wavelength ranges covered by the two PACS settings used for the WILL survey. Table A.3 summarises the properties of the principle transitions observed with PACS towards the WILL sample, while Table A.4 indicates which of these are detected towards each WILL source. The overall fraction of sources detected in each transition is also given in the bottom row of Table A.4. The spectral resolution of PACS is not sufficient to separate the CO $J=31-30$ transition at $84.41 \mu \mathrm{m}$ and the $\mathrm{OH} 84.42 \mu \mathrm{m}$ transition. Emission at this wavelength is therefore marked as a detection for both lines but could be in only $\mathrm{CO}$ or $\mathrm{OH}$. The detection (or not) of neighbouring transitions is likely a reasonable estimate of whether the detected emission is from $\mathrm{CO}, \mathrm{OH}$ or a blend of the two.

\section{A.4. Entrained outflow}

Molecular outflows, usually detected through observations of low- $J{ }^{12} \mathrm{CO}$, are a ubiquitous signpost of ongoing star formation. In order to identify and quantify the properties of the entrained outflowing material associated with WILL sources from ${ }^{12} \mathrm{CO} J=3-2$ maps, a number of steps were taken, following van der Marel et al. (2013):

1. The data were resampled to $0.5 \mathrm{~km} \mathrm{~s}^{-1}$ to improve the sensitivity to line-wing emission.

2. Maps of maximum red and blue-shifted velocities were identified in all spectra as the channel where the emission first reaches $1 \sigma_{\text {rms }}$. 
Table A.1. continued.

\begin{tabular}{|c|c|c|c|c|c|c|c|c|c|}
\hline Name & $\begin{array}{c}70 \\
(\mathrm{Jy}) \\
\end{array}$ & $\begin{array}{l}100 \\
(\mathrm{Jy})\end{array}$ & $\begin{array}{l}160 \\
(\mathrm{Jy})\end{array}$ & $\begin{array}{l}250 \\
(\mathrm{Jy}) \\
\end{array}$ & $\begin{array}{l}350 \\
(\mathrm{Jy}) \\
\end{array}$ & $\begin{array}{r}450^{a} \\
(\mathrm{Jy})\end{array}$ & $\begin{array}{l}500 \\
(\mathrm{Jy}) \\
\end{array}$ & $\begin{array}{r}850^{a} \\
(\mathrm{Jy})\end{array}$ & $\begin{array}{c}1300 \\
(\mathrm{Jy})\end{array}$ \\
\hline AQU 01 & $1.33 \pm 0.03$ & $5.89 \pm 0.08$ & $15.07 \pm 0.25^{b}$ & $19.43 \pm 0.67^{c}$ & $14.85 \pm 0.69^{c}$ & - & $9.87 \pm 0.52^{c}$ & - & $0.65 \pm 0.26$ \\
\hline AQU 02 & $10.88 \pm 0.20$ & $30.77 \pm 0.35$ & $47.80 \pm 1.01^{b}$ & $49.43 \pm 1.23^{c}$ & $36.19 \pm 1.12^{c}$ & - & $22.82 \pm 0.79^{c}$ & - & $0.60 \pm 0.19$ \\
\hline AQU 03 & $2.84 \pm 0.06$ & $4.09 \pm 0.05$ & $7.08 \pm 0.14^{b}$ & $10.94 \pm 0.41^{c}$ & $10.75 \pm 0.60^{c}$ & - & $5.69 \pm 0.48^{c}$ & - & $0.16 \pm 0.06$ \\
\hline AQU 04 & $3.56 \pm 0.09$ & $4.72 \pm 0.07$ & $6.67 \pm 0.17^{b}$ & $20.56 \pm 0.52^{c}$ & $16.71 \pm 0.66^{c}$ & - & $8.98 \pm 0.77^{c}$ & - & $0.23 \pm 0.10$ \\
\hline AQU 05 & $3.21 \pm 0.08$ & $5.95 \pm 0.08$ & $9.45 \pm 0.19^{b}$ & $15.47 \pm 0.49^{c}$ & $11.88 \pm 0.83^{c}$ & - & $6.98 \pm 0.60^{c}$ & - & $0.16 \pm 0.06$ \\
\hline AQU 06 & $1.44 \pm 0.06$ & $2.32 \pm 0.06$ & $4.38 \pm 0.24^{b}$ & $7.44 \pm 0.62^{c}$ & $5.33 \pm 0.62^{c}$ & - & $3.30 \pm 0.50^{c}$ & - & $0.15 \pm 0.06$ \\
\hline CHA 01 & $10.87 \pm 0.23$ & $10.23 \pm 0.14$ & $6.99 \pm 0.17^{b}$ & $3.67 \pm 0.25^{c}$ & $1.58 \pm 0.26^{c}$ & - & - & - & - \\
\hline CHA 02 & $8.87 \pm 0.17$ & $7.48 \pm 0.10$ & $6.25 \pm 0.13^{b}$ & $6.11 \pm 0.43^{c}$ & $4.42 \pm 0.54^{c}$ & - & $4.29 \pm 0.47^{c}$ & - & - \\
\hline CRA 01 & $51.72 \pm 0.94$ & $64.45 \pm 0.65$ & $66.03 \pm 0.81^{b}$ & $51.78 \pm 0.89^{c}$ & $35.52 \pm 0.77^{c}$ & $23.84 \pm 4.77$ & $21.79 \pm 0.51^{c}$ & $3.68 \pm 0.74$ & - \\
\hline OPH 01 & $134.00 \pm 1.60$ & $88.39 \pm 0.78$ & $43.25 \pm 1.18^{b}$ & $11.22 \pm 1.48^{c}$ & - & - & - & - & - \\
\hline ОРН 02 & $98.24 \pm 1.82$ & $103.55 \pm 1.18$ & $71.39 \pm 1.14^{b}$ & $29.44 \pm 0.96^{c}$ & $9.99 \pm 0.76^{c}$ & $4.11 \pm 0.82$ & $2.78 \pm 0.51^{c}$ & $0.85 \pm 0.17$ & - \\
\hline PER 01 & $25.57 \pm 0.51$ & $44.87 \pm 0.55$ & $55.21 \pm 0.90^{b}$ & $40.01 \pm 0.91^{c}$ & $30.67 \pm 0.85^{c}$ & $13.83 \pm 2.77$ & $13.83 \pm 0.56^{c}$ & $2.24 \pm 0.45$ & - \\
\hline PER 02 & $32.16 \pm 0.72$ & $60.76 \pm 0.97$ & $70.74 \pm 2.59^{b}$ & $67.82 \pm 2.70^{c}$ & $47.30 \pm 2.46^{c}$ & - & $23.14 \pm 1.44^{c}$ & - & - \\
\hline PER 04 & $6.80 \pm 0.13$ & $9.36 \pm 0.12$ & $11.72 \pm 0.16^{b}$ & $14.12 \pm 0.20^{c}$ & $10.89 \pm 0.21^{c}$ & - & $5.60 \pm 0.13^{c}$ & $0.63 \pm 0.13$ & - \\
\hline PER 05 & $81.94 \pm 1.59$ & $77.36 \pm 0.92$ & $66.27 \pm 1.03^{b}$ & $44.23 \pm 0.76^{c}$ & $23.63 \pm 0.61^{c}$ & - & $7.93 \pm 0.35^{c}$ & - & - \\
\hline PER 06 & $30.24 \pm 0.84$ & $33.65 \pm 0.63$ & $38.17 \pm 0.72^{b}$ & $21.75 \pm 1.24^{c}$ & - & - & - & - & - \\
\hline PER 07 & $2.30 \pm 0.05$ & $7.75 \pm 0.13$ & $11.47 \pm 0.26^{b}$ & $10.05 \pm 0.32^{c}$ & $9.05 \pm 0.43^{c}$ & $1.38 \pm 0.28$ & $3.38 \pm 0.36^{c}$ & $0.54 \pm 0.11$ & - \\
\hline PER 08 & $40.55 \pm 0.62$ & $66.15 \pm 0.56$ & $102.63 \pm 1.46^{b}$ & $106.89 \pm 2.08^{c}$ & $64.92 \pm 1.97^{c}$ & - & $37.75 \pm 1.27^{c}$ & - & - \\
\hline PER 09 & $60.37 \pm 2.08$ & $55.78 \pm 1.24$ & $40.46 \pm 1.89^{b}$ & $34.99 \pm 2.77^{c}$ & $11.04 \pm 1.34^{c}$ & - & - & - & - \\
\hline PER 10 & $19.80 \pm 0.48$ & $27.83 \pm 0.49$ & $28.43 \pm 1.21^{b}$ & $16.50 \pm 1.31^{c}$ & $8.86 \pm 1.20^{c}$ & $41.51 \pm 8.30$ & $5.77 \pm 0.77^{c}$ & $6.46 \pm 1.29$ & - \\
\hline PER 12 & $2.87 \pm 0.07$ & $6.96 \pm 0.13$ & $11.60 \pm 0.32^{b}$ & $23.94 \pm 0.60^{c}$ & $16.26 \pm 1.09^{c}$ & $13.63 \pm 2.73$ & - & $2.74 \pm 0.55$ & - \\
\hline PER 13 & $3.63 \pm 0.07$ & $6.81 \pm 0.10$ & $9.47 \pm 0.16^{b}$ & $9.07 \pm 0.26^{c}$ & $8.87 \pm 0.31^{c}$ & - & $6.61 \pm 0.24^{c}$ & - & - \\
\hline PER 14 & $8.76 \pm 0.17$ & $8.65 \pm 0.11$ & $9.73 \pm 0.13^{b}$ & $8.88 \pm 0.22^{c}$ & $8.65 \pm 0.29^{c}$ & - & $5.08 \pm 0.21^{c}$ & - & - \\
\hline PER 15 & $7.53 \pm 0.14$ & $18.50 \pm 0.24$ & $26.54 \pm 0.42^{b}$ & $22.04 \pm 0.46^{c}$ & $16.89 \pm 0.46^{c}$ & - & $12.37 \pm 0.32^{c}$ & $2.47 \pm 0.49$ & - \\
\hline PER 16 & $2.90 \pm 0.06$ & $8.34 \pm 0.11$ & $19.21 \pm 0.30^{b}$ & $25.29 \pm 0.52^{c}$ & $26.48 \pm 0.64^{c}$ & $19.55 \pm 3.91$ & $20.97 \pm 0.48^{c}$ & $4.53 \pm 0.91$ & - \\
\hline PER 17 & $1.17 \pm 0.03$ & $1.45 \pm 0.03$ & $1.98 \pm 0.11^{b}$ & - & - & - & - & - & - \\
\hline PER 18 & $2.24 \pm 0.05$ & $6.44 \pm 0.09$ & $9.50 \pm 0.24^{b}$ & $10.88 \pm 0.65^{c}$ & - & - & - & - & - \\
\hline PER 19 & $5.08 \pm 0.10$ & $4.43 \pm 0.07$ & $4.90 \pm 0.12^{b}$ & $4.80 \pm 0.29^{c}$ & $2.55 \pm 0.41^{c}$ & - & - & $1.90 \pm 0.38$ & - \\
\hline PER 20 & $6.18 \pm 0.13$ & $21.36 \pm 0.27$ & $42.63 \pm 0.76^{b}$ & $41.10 \pm 1.49^{c}$ & $30.42 \pm 1.35^{c}$ & $19.88 \pm 3.98$ & $12.88 \pm 0.77^{c}$ & $6.13 \pm 1.23$ & - \\
\hline PER 21 & $5.82 \pm 0.15$ & $15.16 \pm 0.28$ & $17.89 \pm 0.71^{b}$ & $13.60 \pm 0.89^{c}$ & $7.16 \pm 0.84^{c}$ & $18.99 \pm 3.80$ & $3.96 \pm 0.61^{c}$ & $4.04 \pm 0.81$ & - \\
\hline PER 22 & $15.46 \pm 0.31$ & $26.24 \pm 0.40$ & $28.13 \pm 0.83^{b}$ & $21.27 \pm 0.80^{c}$ & $9.88 \pm 0.62^{c}$ & $22.52 \pm 4.50$ & $4.96 \pm 0.36^{c}$ & $2.24 \pm 0.45$ & - \\
\hline SCO 01 & $7.16 \pm 0.15$ & - & $6.57 \pm 0.11^{b}$ & $4.52 \pm 0.15^{c}$ & $2.21 \pm 0.15^{c}$ & - & $0.92 \pm 0.11^{c}$ & $0.16 \pm 0.03$ & - \\
\hline SERS 01 & $34.27 \pm 0.49$ & $56.60 \pm 0.36$ & $84.69 \pm 0.73^{b}$ & $80.67 \pm 1.28^{c}$ & $43.57 \pm 1.30^{c}$ & - & $19.09 \pm 0.92^{c}$ & - & $0.21 \pm 0.05$ \\
\hline SERS 02 & $96.64 \pm 1.39$ & $219.00 \pm 1.75$ & $327.90 \pm 4.45^{b}$ & $283.98 \pm 8.56^{c}$ & $172.40 \pm 6.58^{c}$ & - & $109.94 \pm 3.96^{c}$ & - & $2.50 \pm 0.31$ \\
\hline TAU 01 & $18.67 \pm 0.38$ & - & $24.18 \pm 0.29^{b}$ & $23.52 \pm 0.39^{c}$ & $20.99 \pm 0.43^{c}$ & $30.74 \pm 6.15$ & $13.20 \pm 0.31^{c}$ & $2.38 \pm 0.48$ & - \\
\hline TAU 02 & $2.67 \pm 0.05$ & - & $5.09 \pm 0.06^{b}$ & $4.92 \pm 0.20^{c}$ & $3.28 \pm 0.23^{c}$ & - & $1.46 \pm 0.16^{c}$ & - & - \\
\hline TAU 03 & $4.21 \pm 0.09$ & - & $4.77 \pm 0.09^{b}$ & $2.27 \pm 0.13^{c}$ & $1.27 \pm 0.13^{c}$ & - & $0.63 \pm 0.09^{c}$ & - & - \\
\hline TAU 04 & $17.04 \pm 0.37$ & - & $18.77 \pm 0.33^{b}$ & $15.82 \pm 0.32^{c}$ & $12.66 \pm 0.36^{c}$ & $6.62 \pm 1.32$ & $6.86 \pm 0.24^{c}$ & $1.87 \pm 0.37$ & - \\
\hline TAU 06 & $5.96 \pm 0.12$ & - & $14.23 \pm 0.13^{b}$ & $20.28 \pm 0.28^{c}$ & $13.72 \pm 0.32^{c}$ & - & $6.05 \pm 0.21^{c}$ & - & - \\
\hline TAU 07 & $4.77 \pm 0.09$ & $4.10 \pm 0.05$ & $2.88 \pm 0.06^{b}$ & $1.60 \pm 0.10^{c}$ & $0.89 \pm 0.15^{c}$ & - & $0.59 \pm 0.13^{c}$ & - & - \\
\hline TAU 08 & $3.76 \pm 0.08$ & - & $4.28 \pm 0.07^{b}$ & $3.59 \pm 0.14^{c}$ & $3.35 \pm 0.17^{c}$ & $2.05 \pm 0.41$ & $2.47 \pm 0.14^{c}$ & $0.53 \pm 0.11$ & - \\
\hline TAU 09 & $15.73 \pm 0.26$ & $18.11 \pm 0.17$ & $23.20 \pm 0.24^{b}$ & $17.00 \pm 0.36^{c}$ & $14.60 \pm 0.43^{c}$ & - & $7.46 \pm 0.46^{c}$ & - & - \\
\hline W40 01 & $12.42 \pm 0.95$ & $35.37 \pm 0.88$ & $58.60 \pm 2.35^{b}$ & $62.61 \pm 3.19^{c}$ & $54.51 \pm 2.87^{c}$ & - & $30.67 \pm 1.76^{c}$ & - & $0.50 \pm 0.08$ \\
\hline W40 02 & $24.33 \pm 3.36$ & $26.28 \pm 1.73$ & $42.81 \pm 2.71^{b}$ & $63.53 \pm 3.16^{c}$ & $60.78 \pm 2.87^{c}$ & - & $39.55 \pm 1.80^{c}$ & - & $0.90 \pm 0.24$ \\
\hline W40 03 & - & - & $120.48 \pm 3.55^{b}$ & $106.22 \pm 3.83^{c}$ & $98.41 \pm 3.34^{c}$ & - & $51.79 \pm 1.94^{c}$ & - & $0.95 \pm 0.29$ \\
\hline W40 04 & - & - & $95.75 \pm 3.06^{b}$ & $108.24 \pm 3.46^{c}$ & $37.51 \pm 3.02^{c}$ & - & $15.47 \pm 1.72^{c}$ & - & $0.60 \pm 0.14$ \\
\hline W40 05 & - & - & $71.84 \pm 2.85^{b}$ & $75.62 \pm 3.70^{c}$ & $61.72 \pm 5.65^{c}$ & - & $62.30 \pm 7.37^{c}$ & - & $0.76 \pm 0.23$ \\
\hline W40 06 & - & $1.68 \pm 0.09$ & $7.01 \pm 0.40^{b}$ & $12.62 \pm 0.91^{c}$ & $8.58 \pm 0.85^{c}$ & - & $1.33 \pm 0.77^{c}$ & - & $0.20 \pm 0.06$ \\
\hline W40 07 & $3.18 \pm 0.07$ & $7.01 \pm 0.10$ & $10.72 \pm 0.28^{b}$ & $8.76 \pm 0.53^{c}$ & $7.58 \pm 0.95^{c}$ & - & $3.35 \pm 0.49^{c}$ & - & $0.09 \pm 0.03$ \\
\hline
\end{tabular}

3. The outer velocity $\left(v_{\text {out }}\right)$ of each outflow lobe was defined from the maximum velocity maps as the most offset value from the source velocity. The maximum velocity $\left(v_{\max }\right)$ is the absolute difference between $v_{\text {out }}$ and $v_{\text {LSR }}$.

4. The inner velocity $\left(v_{\text {in }}\right)$ for each lobe was defined by the same approach using a spectrum without any outflow emission, so as to mask out cloud emission.

5. Integrated maps for the red and blue outflow lobes were created by integrating between the minimum and maximum velocities. Visual inspection and comparison of these maps with the continuum emission was then used to identify the spectra associated with an outflow from the source. Excluding low-velocity emission will lead to an underestimate in the mass and related properties by factors of a few (Downes \& Cabrit 2007). However, this is generally preferable to performing an incorrect correction based on poor knowledge of the contribution from the envelope (Cabrit \& Bertout 1990).

6. Each source was assigned an inclination $(i)$ of $10,30,50$ or $70^{\circ}$ between the outflow axis and the line of sight, such that $i=0^{\circ}$ is pole-on, through visual inspection of the overlap between the red and blue lobes with each other and the source position.

7. The radius associated with each outflow lobe $\left(R_{\mathrm{CO}}\right)$ was defined as the distance between the source position and furthest pixel containing outflow emission.

8. The mass in each channel of each pixel $\left(m_{i j}\right)$ was calculated assuming an excitation temperature $\left(T_{\mathrm{ex}}\right)$ of $75 \mathrm{~K}, \mu=2.8$ (Kauffmann et al. 2008) and a CO abundance relative to $\mathrm{H}_{2}$ of $1.2 \times 10^{-4}$ (Frerking et al. 1982), consistent with the 
Table A.2. Pacs spectral continuum fluxes.

\begin{tabular}{|c|c|c|c|c|c|c|c|c|c|c|c|c|}
\hline Name & 63.2 & 79.2 & 81.8 & 84.6 & 90.0 & 108.8 & 125.4 & 157.7 & 164.0 & 169.1 & 179.5 & 190.0 \\
\hline AQU 01 & 3.8 & 4.5 & 3.8 & 3.6 & 5.5 & 9.7 & 11.3 & 18.5 & 18.2 & 17.5 & 20.4 & 18.5 \\
\hline AQU 02 & 8.4 & 10.1 & 12.2 & 13.1 & 15.1 & 31.3 & 34.9 & 46.5 & 46.6 & 44.4 & 46.3 & 39.9 \\
\hline AQU 03 & 3.7 & 2.5 & 2.2 & 1.8 & 2.4 & 3.8 & 2.9 & 4.8 & 6.2 & 5.4 & 8.5 & 8.6 \\
\hline AQU 04 & 5.8 & 2.9 & 3.6 & 3.9 & 4.0 & 4.1 & 4.0 & 6.4 & 7.4 & 8.6 & 10.5 & 13.2 \\
\hline AQU 05 & 3.2 & 1.8 & 2.3 & 2.2 & 2.7 & 4.3 & 3.9 & 7.2 & 8.7 & 9.1 & 11.2 & 12.7 \\
\hline AQU $06^{a, b}$ & - & - & - & - & - & - & - & - & - & - & - & - \\
\hline CHA 01 & 14.7 & 9.7 & 9.0 & 10.2 & 11.2 & 22.0 & 13.8 & 16.6 & 16.6 & 15.8 & 15.4 & 21.7 \\
\hline CHA 02 & 10.9 & 9.4 & 8.3 & 7.6 & 9.3 & 14.3 & 9.3 & 13.0 & 13.6 & 13.8 & 13.8 & 14.5 \\
\hline CRA 01 & 49.4 & 57.7 & 56.9 & 58.4 & 62.5 & 71.5 & 62.9 & 65.2 & 60.7 & 55.9 & 54.5 & 45.5 \\
\hline $\mathrm{OPH} 01^{a}$ & 139.1 & 157.8 & 161.3 & 161.5 & 159.7 & 161.0 & 137.4 & 111.0 & 102.6 & 94.0 & 86.7 & 73.4 \\
\hline OPH 02 & 84.7 & 98.7 & 99.3 & 102.2 & 101.9 & 102.5 & 84.6 & 75.8 & 69.4 & 64.3 & 59.1 & 49.1 \\
\hline PER 01 & 20.7 & 31.7 & 35.3 & 38.0 & 42.8 & 57.7 & 58.1 & 68.6 & 65.9 & 62.4 & 60.4 & 50.2 \\
\hline $\mathrm{R} 02$ & 29.1 & 55.7 & 60.1 & 66.4 & 78.6 & 122.1 & 139.4 & 186.0 & 182.9 & 177.1 & 175.8 & 141.7 \\
\hline PER 04 & 5.7 & 7.1 & 6.7 & 8.4 & 8.4 & 11.1 & 9.5 & 11.6 & 11.1 & 10.1 & 10.6 & 9.6 \\
\hline PER 05 & 67.1 & 67.8 & 67.3 & 70.0 & 71.7 & 75.3 & 65.2 & 65.3 & 62.0 & 57.5 & 54.0 & 45.0 \\
\hline PER 06 & 41.4 & 50.2 & 48.9 & 48.1 & 49.3 & 71.2 & 59.3 & 65.1 & 62.0 & 56.6 & 56.5 & 51.2 \\
\hline R 07 & 2.8 & 2.5 & 3.2 & 4.0 & 5.7 & 9.7 & 9.0 & 13.1 & 13.1 & 11.9 & 13.2 & 10.9 \\
\hline PER 08 & 31.2 & 47.0 & 51.0 & 55.2 & 65.6 & 109.9 & 110.8 & 147.7 & 146.5 & 144.2 & 141.9 & 120.3 \\
\hline PER $09^{a}$ & 242.5 & 291.1 & 301.7 & 308.8 & 324.4 & 398.7 & 357.1 & 310.7 & 281.6 & 260.8 & 227.9 & 190.6 \\
\hline PER 10 & 34.4 & 47.8 & 52.3 & 53.3 & 58.4 & 88.3 & 80.9 & 88.4 & 84.3 & 80.6 & 78.6 & 68.9 \\
\hline R 12 & 2.6 & 3.6 & 3.3 & 3.4 & 6.1 & 14.1 & 14.6 & 21.0 & 20.8 & 20.5 & 22.8 & 24.0 \\
\hline R 13 & 3.2 & 2.1 & 2.7 & 2.9 & 3.9 & 9.2 & 7.0 & 8.9 & 8.9 & 7.8 & 9.3 & 8.8 \\
\hline PER 14 & 9.9 & 9.0 & 7.3 & 6.5 & 7.9 & 11.4 & 8.5 & 9.1 & 10.0 & 9.3 & 11.3 & 10.0 \\
\hline PER 15 & 5.4 & 11.4 & 11.9 & 12.6 & 16.6 & 24.9 & 23.0 & 27.5 & 27.7 & 26.5 & 26.0 & 21.7 \\
\hline PER 16 & 2.7 & 5.1 & 6.4 & 6.4 & 8.9 & 11.8 & 11.9 & 18.6 & 19.5 & 18.3 & 22.5 & 18.2 \\
\hline PER $17^{a}$ & 2.7 & 3.1 & 1.9 & 2.3 & 2.8 & 5.7 & 6.2 & 14.1 & 14.1 & 15.3 & 16.2 & 16.4 \\
\hline PER $18^{a}$ & 3.0 & 5.1 & 5.3 & 5.5 & 8.1 & 11.5 & 12.0 & 20.3 & 19.6 & 20.0 & 20.4 & 20.5 \\
\hline PER $19^{a}$ & 0.9 & 3.2 & 2.5 & 3.1 & 3.5 & 6.7 & 4.5 & 7.9 & 7.0 & 6.8 & 8.4 & 10.8 \\
\hline PER 20 & 2.0 & 7.3 & 9.5 & 11.9 & 15.6 & 33.9 & 43.2 & 65.0 & 66.8 & 63.6 & 64.6 & 54.3 \\
\hline PER 21 & 5.6 & 11.1 & 13.0 & 13.4 & 17.0 & 32.8 & 34.8 & 49.8 & 49.0 & 46.7 & 46.9 & 42.8 \\
\hline PER 22 & 10.3 & 15.8 & 18.2 & 19.2 & 21.5 & 29.2 & 31.5 & 40.9 & 43.5 & 40.8 & 41.2 & 32.7 \\
\hline SCO 01 & 8.4 & 4.9 & 5.0 & 5.9 & 6.5 & 5.0 & 2.9 & 2.8 & 2.3 & 1.5 & 2.8 & 1.6 \\
\hline SERS 01 & 18.4 & 28.6 & 29.9 & 32.6 & 35.7 & 59.8 & 63.9 & 77.5 & 74.9 & 72.2 & 70.8 & 60.4 \\
\hline SERS 02 & 58.8 & 134.4 & 148.5 & 163.4 & 193.3 & 327.2 & 370.8 & 452.4 & 440.3 & 425.2 & 411.8 & 333.8 \\
\hline TAU 01 & 18.8 & 17.4 & 17.4 & 17.0 & 18.2 & 23.6 & 21.4 & 26.4 & 25.5 & 24.1 & 23.7 & 19.9 \\
\hline TAU 02 & 3.4 & 4.4 & 3.8 & 3.6 & 4.4 & 5.9 & 4.7 & 7.6 & 6.6 & 7.0 & 8.8 & 7.7 \\
\hline TAU 03 & 3.9 & 4.5 & 4.8 & 4.1 & 5.2 & 7.0 & 4.6 & 4.6 & 4.0 & 4.5 & 4.8 & 4.0 \\
\hline TAU 04 & 15.9 & 16.9 & 16.6 & 17.0 & 17.1 & 24.2 & 19.9 & 22.7 & 23.0 & 21.1 & 19.7 & 18.3 \\
\hline TAU 06 & 7.4 & 4.9 & 5.8 & 6.5 & 7.8 & 13.4 & 12.2 & 14.6 & 15.3 & 13.7 & 15.7 & 14.1 \\
\hline TAU 07 & 8.4 & 4.9 & 5.0 & 5.9 & 6.5 & 5.0 & 2.9 & 2.8 & 2.3 & 1.5 & 2.8 & 1.6 \\
\hline TAU $08^{c}$ & - & - & - & - & - & - & - & - & - & - & - & - \\
\hline TAU 09 & 14.7 & 17.3 & 19.5 & 19.9 & 22.0 & 22.0 & 20.4 & 23.3 & 22.8 & 22.6 & 23.1 & 21.7 \\
\hline $\mathrm{W} 4001^{a}$ & 36.7 & 67.3 & 77.2 & 84.7 & 103.8 & 193.8 & 226.6 & 274.0 & 267.7 & 252.9 & 240.7 & 193.0 \\
\hline $\mathrm{W} 4002^{a}$ & 537.6 & 455.5 & 454.6 & 446.9 & 431.4 & 400.4 & 324.3 & 267.7 & 247.2 & 230.2 & 209.5 & 180.1 \\
\hline $\mathrm{W} 4003^{a}$ & 128.8 & 204.8 & 219.6 & 230.9 & 251.7 & 352.6 & 353.5 & 363.1 & 339.4 & 316.0 & 288.7 & 238.3 \\
\hline $\mathrm{W} 4004^{a}$ & 208.7 & 303.3 & 323.7 & 340.3 & 372.4 & 496.3 & 465.4 & 429.8 & 395.4 & 363.5 & 324.5 & 268.3 \\
\hline $\mathrm{W} 4005^{a}$ & 106.8 & 173.7 & 187.8 & 200.3 & 228.2 & 343.1 & 346.4 & 362.9 & 335.7 & 316.1 & 291.6 & 238.9 \\
\hline $\mathrm{W} 4006^{a}$ & - & - & - & - & - & - & - & - & - & 0.1 & 6.3 & 8.5 \\
\hline W40 07 & 3.9 & 3.8 & 5.0 & 5.3 & 8.0 & 14.4 & 17.0 & 25.9 & 27.4 & 24.8 & 27.4 & 22.2 \\
\hline
\end{tabular}

Notes. All fluxes are given in Jy, all numerical wavelengths in $\mu \mathrm{m} .{ }^{(a)}$ Data not included in SED. ${ }^{(b)}$ Continuum unreliable due to contamination of reference positions. ${ }^{(c)}$ Source not observed with PACS.

outflow properties determined by Yildiz et al. (2015) for the WISH sample. Changing $T_{\text {ex }}$ to 100 or $50 \mathrm{~K}$ would only raise or lower the mass by a factor of 1.2. No correction for $\tau$ is performed, as the optically thick parts of the line near the line centre are excluded and the line wings are typically optically thin in outflows associated with low-mass protostars (see van der Marel et al. 2013; Yıldız et al. 2015).
9. The physical properties of the outflow were calculated using the separation method (M7 in van der Marel et al. 2013), where the mass, momentum and energy are calculated separately for each lobe on a per-channel basis for each spectrum, then summed over all channels and spectra, while the maximum velocity is used to calculate the dynamical time of the 
J. C. Mottram et al.: Outflows, infall and evolution of a sample of embedded low-mass protostars

Table A.3. Principle lines observed with PACS.

\begin{tabular}{|c|c|c|c|c|c|c|c|c|}
\hline Species & Transition & $\begin{array}{l}\text { Rest frequency }{ }^{a} \\
(\mathrm{GHz})\end{array}$ & $\begin{array}{l}\text { Wavelength } \\
(\mu \mathrm{m})\end{array}$ & $\begin{array}{c}E_{\mathrm{u}} / k_{\mathrm{b}} \\
(\mathrm{K})\end{array}$ & $\begin{array}{l}A_{\mathrm{ul}}{ }^{b} \\
\left(\mathrm{~s}^{-1}\right) \\
\end{array}$ & $\begin{array}{c}n_{\mathrm{cr}} \\
\left(\mathrm{cm}^{-3}\right)\end{array}$ & $\begin{array}{r}\theta_{\mathrm{mb}}{ }^{c} \\
\left({ }^{\prime \prime}\right)\end{array}$ & $\begin{array}{l}\text { Obs. time }^{d} \\
(\mathrm{~min})\end{array}$ \\
\hline $\mathrm{o}-\mathrm{H}_{2} \mathrm{O}$ & $\begin{array}{l}2_{12}-1_{01} \\
2_{21}-2_{12} \\
2_{21}-1_{10} \\
4_{23}-3_{12} \\
6_{16}-5_{05} \\
7_{16}-7_{07} \\
8_{18}-7_{07} \\
\end{array}$ & $\begin{array}{l}1669.90477 \\
1661.00764 \\
2773.97659 \\
3807.25841 \\
3654.60328 \\
3536.66681 \\
4734.29617 \\
\end{array}$ & $\begin{array}{r}179.527 \\
180.488 \\
108.073 \\
78.742 \\
82.031 \\
84.770 \\
63.323 \\
\end{array}$ & \begin{tabular}{r|}
114.4 \\
194.1 \\
194.1 \\
432.2 \\
643.5 \\
1013.2 \\
1070.7 \\
\end{tabular} & $\begin{array}{l}5.59 \times 10^{-2} \\
3.06 \times 10^{-2} \\
2.56 \times 10^{-1} \\
4.84 \times 10^{-1} \\
7.49 \times 10^{-1} \\
2.16 \times 10^{-1} \\
1.75 \times 10^{0} \\
\end{array}$ & $\begin{array}{l}3 \times 10^{8} \\
1 \times 10^{8} \\
1 \times 10^{9} \\
2 \times 10^{9} \\
4 \times 10^{9} \\
1 \times 10^{9} \\
1 \times 10^{10} \\
\end{array}$ & $\begin{array}{r}12.7 \\
12.8 \\
7.6 \\
5.6 \\
5.8 \\
6.0 \\
4.5 \\
\end{array}$ & $\begin{array}{l}36 \\
36 \\
17 \\
36 \\
36 \\
36 \\
17 \\
\end{array}$ \\
\hline $\mathrm{p}-\mathrm{H}_{2} \mathrm{O}$ & $\begin{array}{l}3_{22}-2_{11} \\
4_{04}-3_{13} \\
6_{15}-5_{24} \\
\end{array}$ & $\begin{array}{l}3331.45838 \\
2391.57263 \\
3798.28164 \\
\end{array}$ & $\begin{array}{r}89.988 \\
125.354 \\
78.928 \\
\end{array}$ & $\begin{array}{l}296.8 \\
319.5 \\
781.1 \\
\end{array}$ & $\begin{array}{l}3.52 \times 10^{-1} \\
1.73 \times 10^{-1} \\
4.52 \times 10^{-1} \\
\end{array}$ & $\begin{array}{l}1 \times 10^{9} \\
9 \times 10^{8} \\
2 \times 10^{9} \\
\end{array}$ & $\begin{array}{l}6.4 \\
8.9 \\
5.6 \\
\end{array}$ & $\begin{array}{l}36 \\
36 \\
36 \\
\end{array}$ \\
\hline $\mathrm{OH}$ & $\begin{array}{l}1 / 2,1 / 2-3 / 2,3 / 2 \\
1 / 2,1 / 2-3 / 2,3 / 2 \\
3 / 2,1 / 2-1 / 2,1 / 2 \\
3 / 2,1 / 2-1 / 2,1 / 2 \\
7 / 2,3 / 2-5 / 2,3 / 2 \\
7 / 2,3 / 2-5 / 2,3 / 2\end{array}$ & $\begin{array}{l}3786.16998 \\
3789.17979 \\
1834.74735 \\
1837.81682 \\
3543.77937 \\
3551.18525 \\
\end{array}$ & \begin{tabular}{r|}
79.181 \\
79.118 \\
163.397 \\
163.124 \\
84.597 \\
84.420 \\
\end{tabular} & $\begin{array}{l}181.7 \\
181.9 \\
269.8 \\
270.2 \\
290.5 \\
291.2\end{array}$ & $\begin{array}{l}3.52 \times 10^{-2} \\
3.49 \times 10^{-2} \\
6.37 \times 10^{-2} \\
6.40 \times 10^{-2} \\
5.13 \times 10^{-1} \\
5.16 \times 10^{-1}\end{array}$ & $\begin{array}{l}1 \times 10^{8} \\
8 \times 10^{7} \\
2 \times 10^{8} \\
2 \times 10^{8} \\
2 \times 10^{9} \\
2 \times 10^{9}\end{array}$ & $\begin{array}{r}5.6 \\
5.6 \\
11.6 \\
11.5 \\
6.0 \\
6.0\end{array}$ & $\begin{array}{l}36 \\
36 \\
36 \\
36 \\
36 \\
36\end{array}$ \\
\hline [O I $]$ & ${ }^{3} \mathrm{P}_{1}-{ }^{3} \mathrm{P}_{2}$ & 4744.77749 & 63.184 & 227.7 & $8.91 \times 10^{-5}$ & $5 \times 10^{5}$ & 4.5 & 17 \\
\hline $\mathrm{CO}$ & $\begin{array}{l}16-15 \\
21-20 \\
24-23 \\
29-28 \\
31-30 \\
32-31 \\
33-32 \\
\end{array}$ & $\begin{array}{l}1841.34551 \\
2413.91711 \\
2756.38758 \\
3325.00528 \\
3551.59236 \\
3664.68418 \\
3777.63573 \\
\end{array}$ & $\begin{array}{r}162.812 \\
124.193 \\
108.763 \\
90.163 \\
84.411 \\
81.806 \\
79.360 \\
\end{array}$ & $\begin{array}{r}751.7 \\
1276.1 \\
1656.5 \\
2399.8 \\
2735.3 \\
2911.2 \\
3092.5 \\
\end{array}$ & $\begin{array}{l}4.05 \times 10^{-4} \\
8.83 \times 10^{-4} \\
1.28 \times 10^{-3} \\
2.13 \times 10^{-3} \\
2.52 \times 10^{-3} \\
2.74 \times 10^{-3} \\
2.95 \times 10^{-3} \\
\end{array}$ & $\begin{array}{l}1 \times 10^{6} \\
2 \times 10^{6} \\
3 \times 10^{6} \\
6 \times 10^{6} \\
7 \times 10^{6} \\
7 \times 10^{6} \\
8 \times 10^{6} \\
\end{array}$ & $\begin{array}{r}11.5 \\
8.8 \\
7.7 \\
6.4 \\
6.0 \\
5.8 \\
5.6 \\
\end{array}$ & $\begin{array}{l}36 \\
36 \\
17 \\
36 \\
36 \\
36 \\
36 \\
\end{array}$ \\
\hline [C II] & ${ }^{2} \mathrm{P}_{3 / 2}-{ }^{2} \mathrm{P}_{1 / 2}$ & 1900.53690 & 157.741 & 91.2 & $2.32 \times 10^{-6}$ & $3 \times 10^{3}$ & 11.2 & 36 \\
\hline
\end{tabular}

Notes. ${ }^{(a)}$ Taken from the JPL database (Pickett et al. 2010) for $\mathrm{H}_{2} \mathrm{O}, \mathrm{OH}$ and CO, Zink et al. (1991) for [O I] and CDMS (Müller et al. 2001, 2005) for [C II]. ${ }^{(b)}$ Taken from Daniel et al. (2011) and Dubernet et al. (2009) for $\mathrm{H}_{2} \mathrm{O}$, the JPL database (Pickett et al. 2010) for OH and CO, Fischer \& Saha (1983) for [O I] and CDMS (Müller et al. 2001, 2005) for [CII]. ${ }^{(c)}$ Calculated using Eq. (3) from Roelfsema et al. (2012). ${ }^{(d)}$ Total time including on+off source and overheads.

flow, that is:

$$
\begin{aligned}
& M_{\text {out }}=\sum_{j=1}^{\text {npix }} \sum_{v_{\text {in }}}^{v_{\text {out }}} m_{i, j} \mathrm{~d} v \\
& P_{\text {out }}=\sum_{j=1}^{\text {npix }} \sum_{v_{\text {in }}}^{v_{\text {out }}} m_{i, j}\left|v_{i}-v_{\mathrm{LSR}}\right| \mathrm{d} v, \\
& E_{\text {out }}=\sum_{j=1}^{\text {npix }} \sum_{v_{\text {in }}}^{v_{\text {out }}} m_{i, j}\left|v_{i}-v_{\mathrm{LSR}}\right|^{2} \mathrm{~d} v, \\
& t_{\text {dyn }}=\frac{R_{\mathrm{CO}}}{v_{\text {max }}} \\
& \dot{M}_{\text {out }}=\frac{c_{\mathrm{f}} M_{\text {out }}}{t_{\text {dyn }}}
\end{aligned}
$$

and

$$
F_{\mathrm{CO}}=\frac{c_{\mathrm{f}} P_{\mathrm{out}}}{t_{\mathrm{dyn}}},
$$

where $c_{\mathrm{f}}$ is a correction factor to account for inclination, given in Table A.5 and derived from the models of Downes \& Cabrit (2007) by van der Marel et al. (2013).
Table A.6 gives the calculated outflow properties for the red and blue lobes separately, including the velocity limits, outflow mass $\left(M_{\text {out }}\right)$, momentum $\left(P_{\text {out }}\right)$, kinetic energy $\left(E_{\text {out }}\right)$, radius, inclination, dynamical time $\left(t_{\text {dyn }}\right)$, force $\left(F_{\mathrm{CO}}\right)$ and mass outflow rate $\left(\dot{M}_{\text {out }}\right)$. In some cases, the outflow may extend beyond the $2^{\prime} \times 2^{\prime}$ coverage of the observations, so these values may be lower limits to the total value. Figure A.2 shows the outflow lobes of the observed outflows associated with the WILL sources overlaid on the Herschel PACS 70 micron maps.

\section{A.5. Mass accretion and loss}

Table A.7 presents the calculated mass accretion rates for all WILL, WISH and DIGIT sources, calculated as discussed in Sect. 4.4 using Eq. (6), as well as the observed luminosity in the [O I] $63 \mu \mathrm{m}$ transition and the mass-loss rate in the wind derived using the relation from Hollenbach (1985), given in Eq. (7). $L[\mathrm{OI} 63 \mu \mathrm{m}]$ is not given for OPH 01 , W40 01 and W40 03-06 as the detections in $[\mathrm{O} \mathrm{I}]$ towards these sources are almost certainly due to PDR emission.

\section{A.6. Ground-based line fitting}

Table A.8 presents the basic properties of all transitions observed with the JCMT and APEX. Gaussian fitting was performed for 
Table A.4. PACS line detections.

\begin{tabular}{|c|c|c|c|c|c|c|c|c|c|c|c|}
\hline Name & $2{ }_{12}-1_{01}$ & $2_{21}-2_{12}$ & $2_{21}-1_{10}$ & $\begin{array}{l}\mathrm{O}-\mathrm{H}_{2} \mathrm{O} \\
4_{23}-3_{12}\end{array}$ & $6_{16}-5_{05}$ & $7_{16}-7_{07}$ & $8_{18}-7_{07}$ & $3_{22}-2_{11}$ & $\begin{array}{l}\mathrm{p}-\mathrm{H}_{2} \mathrm{O} \\
4_{04}-3_{13}\end{array}$ & $6_{15}-5_{24}$ & $\begin{array}{c}{[\mathrm{OI}]} \\
{ }^{3} \mathrm{P}_{1}-{ }^{3} \mathrm{P}_{2} \\
\end{array}$ \\
\hline AQU 01 & $\mathrm{Y}$ & $\mathrm{Y}$ & $\mathrm{Y}$ & $\mathrm{N}$ & $\mathrm{N}$ & $\mathrm{N}$ & $\mathrm{N}$ & $\mathrm{N}$ & $\mathrm{Y}$ & $\mathrm{N}$ & $\mathrm{Y}$ \\
\hline AQU 02 & $\mathrm{Y}$ & $\mathrm{Y}$ & $\mathrm{Y}$ & $\mathrm{N}$ & $\mathrm{N}$ & $\mathrm{N}$ & $\mathrm{N}$ & $\mathrm{Y}$ & $Y$ & $\mathrm{~N}$ & Y \\
\hline AQU 03 & $\mathrm{~N}$ & $\mathrm{~N}$ & $\mathrm{~N}$ & $\mathrm{~N}$ & $\mathrm{~N}$ & $\mathrm{~N}$ & $\mathrm{~N}$ & $\mathrm{~N}$ & $\mathrm{~N}$ & $\mathrm{~N}$ & $\mathrm{~N}$ \\
\hline AQU 04 & $\mathrm{~N}$ & $\mathrm{~N}$ & $\mathrm{~N}$ & $\mathrm{~N}$ & $\mathrm{~N}$ & $\mathrm{~N}$ & $\mathrm{~N}$ & $\mathrm{~N}$ & $\mathrm{~N}$ & $\mathrm{~N}$ & $\mathrm{~N}$ \\
\hline AQU 05 & $\mathrm{Y}$ & $\mathrm{N}$ & $\mathrm{Y}$ & $\mathrm{N}$ & $\mathrm{N}$ & $\mathrm{N}$ & $\mathrm{N}$ & $\mathrm{N}$ & $\mathrm{N}$ & $\mathrm{N}$ & $\mathrm{N}$ \\
\hline AQU 06 & $\mathrm{~N}$ & $\mathrm{~N}$ & $\mathrm{~N}$ & $\mathrm{~N}$ & $\mathrm{~N}$ & $\mathrm{~N}$ & $\mathrm{~N}$ & $\mathrm{~N}$ & $\mathrm{~N}$ & $\mathrm{~N}$ & $\mathrm{~N}$ \\
\hline CHA 01 & $\mathrm{Y}$ & $\mathrm{N}$ & $\mathrm{Y}$ & $\mathrm{Y}$ & Y & $\mathrm{N}$ & $\mathrm{N}$ & $\mathrm{Y}$ & $\mathrm{Y}$ & $\mathrm{N}$ & $\mathrm{Y}$ \\
\hline CHA 02 & $\mathrm{~N}$ & $\mathrm{~N}$ & Y & Y & Y & $\mathrm{N}$ & $\mathrm{N}$ & $\mathrm{N}$ & $\mathrm{N}$ & Y & Y \\
\hline CRA 01 & $\mathrm{Y}$ & $\mathrm{N}$ & $Y$ & $\mathrm{Y}$ & $\mathrm{N}$ & $\mathrm{N}$ & $\mathrm{N}$ & Y & $\mathrm{Y}$ & $\mathrm{N}$ & Y \\
\hline ОРН 01 & $\mathrm{Y}$ & $\mathrm{N}$ & $\mathrm{N}$ & $\mathrm{N}$ & $\mathrm{N}$ & $\mathrm{N}$ & $\mathrm{N}$ & $\mathrm{N}$ & $\mathrm{N}$ & $\mathrm{N}$ & $\mathrm{Y}$ \\
\hline ОРН 02 & $\mathrm{Y}$ & $\mathrm{Y}$ & $\mathrm{Y}$ & $\mathrm{Y}$ & Y & $\mathrm{N}$ & $\mathrm{Y}$ & $\mathrm{Y}$ & $\mathrm{Y}$ & $\mathrm{Y}$ & Y \\
\hline PER 01 & $\mathrm{Y}$ & $\mathrm{Y}$ & $\mathrm{Y}$ & $\mathrm{Y}$ & Y & $\mathrm{N}$ & $\mathrm{Y}$ & $\mathrm{Y}$ & $\mathrm{Y}$ & $\mathrm{Y}$ & $\mathrm{Y}$ \\
\hline PER 02 & Y & Y & Y & Y & Y & $\mathrm{N}$ & Y & Y & Y & Y & Y \\
\hline PER 04 & $\mathrm{~N}$ & $\mathrm{~N}$ & $\mathrm{~N}$ & $\mathrm{~N}$ & $\mathrm{~N}$ & $\mathrm{~N}$ & $\mathrm{~N}$ & $\mathrm{~N}$ & $\mathrm{~N}$ & $\mathrm{~N}$ & $\mathrm{Y}$ \\
\hline PER 05 & $\mathrm{Y}$ & $\mathrm{Y}$ & $\mathrm{Y}$ & Y & Y & $\mathrm{N}$ & $\mathrm{N}$ & Y & Y & Y & Y \\
\hline PER 06 & $\mathrm{Y}$ & $\mathrm{N}$ & $\mathrm{Y}$ & $\mathrm{Y}$ & $\mathrm{Y}$ & $\mathrm{N}$ & $\mathrm{Y}$ & $\mathrm{N}$ & $\mathrm{Y}$ & $\mathrm{N}$ & Y \\
\hline PER 07 & $\mathrm{~N}$ & $\mathrm{~N}$ & $\mathrm{~N}$ & $\mathrm{~N}$ & $\mathrm{~N}$ & $\mathrm{~N}$ & $\mathrm{~N}$ & $\mathrm{~N}$ & $\mathrm{~N}$ & $\mathrm{~N}$ & Y \\
\hline PER 08 & $\mathrm{Y}$ & $\mathrm{N}$ & $\mathrm{Y}$ & Y & Y & $\mathrm{N}$ & Y & Y & Y & $\mathrm{N}$ & Y \\
\hline PER 09 & Y & $\mathrm{Y}$ & $\mathrm{Y}$ & $\mathrm{Y}$ & $\mathrm{Y}$ & $\mathrm{N}$ & Y & $\mathrm{Y}$ & $Y$ & Y & Y \\
\hline PER 10 & $\mathrm{Y}$ & $\mathrm{N}$ & $\mathrm{Y}$ & $\mathrm{N}$ & $\mathrm{N}$ & $\mathrm{N}$ & $\mathrm{N}$ & $\mathrm{N}$ & $\mathrm{Y}$ & $\mathrm{N}$ & Y \\
\hline PER 12 & $\mathrm{~N}$ & $\mathrm{~N}$ & $\mathrm{~N}$ & $\mathrm{~N}$ & $\mathrm{~N}$ & $\mathrm{~N}$ & $\mathrm{~N}$ & $\mathrm{~N}$ & $\mathrm{~N}$ & $\mathrm{~N}$ & Y \\
\hline PER 13 & $\mathrm{~N}$ & $\mathrm{~N}$ & $\mathrm{Y}$ & $\mathrm{N}$ & $\mathrm{N}$ & $\mathrm{N}$ & $\mathrm{N}$ & $\mathrm{N}$ & $\mathrm{Y}$ & $\mathrm{N}$ & $\mathrm{N}$ \\
\hline PER 14 & $\mathrm{Y}$ & $\mathrm{Y}$ & $\mathrm{Y}$ & $\mathrm{Y}$ & $\mathrm{Y}$ & $\mathrm{N}$ & $\mathrm{Y}$ & $\mathrm{Y}$ & $\mathrm{Y}$ & $\mathrm{N}$ & $\mathrm{Y}$ \\
\hline PER 15 & $\mathrm{Y}$ & $\mathrm{N}$ & $\mathrm{Y}$ & $\mathrm{N}$ & $\mathrm{Y}$ & $\mathrm{N}$ & $\mathrm{N}$ & $\mathrm{N}$ & $Y$ & $\mathrm{~N}$ & $\mathrm{Y}$ \\
\hline PER 16 & $\mathrm{~N}$ & $\mathrm{~N}$ & $\mathrm{~N}$ & $\mathrm{~N}$ & $\mathrm{~N}$ & $\mathrm{~N}$ & $\mathrm{~N}$ & $\mathrm{~N}$ & $\mathrm{~N}$ & $\mathrm{~N}$ & Y \\
\hline PER 17 & $\mathrm{~N}$ & $\mathrm{~N}$ & $\mathrm{~N}$ & $\mathrm{~N}$ & $\mathrm{~N}$ & $\mathrm{~N}$ & $\mathrm{~N}$ & $\mathrm{~N}$ & $\mathrm{~N}$ & $\mathrm{~N}$ & Y \\
\hline PER 18 & Y & $\mathrm{Y}$ & $\mathrm{Y}$ & $\mathrm{N}$ & $\mathrm{N}$ & $\mathrm{N}$ & $\mathrm{N}$ & $\mathrm{N}$ & $\mathrm{N}$ & $\mathrm{N}$ & Y \\
\hline PER 19 & $\mathrm{Y}$ & $\mathrm{Y}$ & $\mathrm{Y}$ & $\mathrm{N}$ & $\mathrm{Y}$ & $\mathrm{N}$ & $\mathrm{N}$ & $\mathrm{N}$ & $\mathrm{Y}$ & $\mathrm{N}$ & $\mathrm{Y}$ \\
\hline PER 20 & $\mathrm{Y}$ & $\mathrm{Y}$ & $\mathrm{Y}$ & $\mathrm{Y}$ & $\mathrm{N}$ & $\mathrm{N}$ & $\mathrm{N}$ & $\mathrm{N}$ & $\mathrm{Y}$ & $\mathrm{N}$ & Y \\
\hline PER 21 & $\mathrm{Y}$ & $\mathrm{Y}$ & $\mathrm{Y}$ & $\mathrm{Y}$ & $\mathrm{Y}$ & $\mathrm{N}$ & $\mathrm{N}$ & $\mathrm{Y}$ & Y & $\mathrm{N}$ & Y \\
\hline PER 22 & $\mathrm{Y}$ & $\mathrm{Y}$ & $\mathrm{Y}$ & $\mathrm{Y}$ & $\mathrm{Y}$ & $\mathrm{N}$ & $\mathrm{N}$ & $\mathrm{N}$ & $Y$ & $\mathrm{~N}$ & Y \\
\hline SCO 01 & $\mathrm{Y}$ & $\mathrm{N}$ & $\mathrm{Y}$ & $\mathrm{Y}$ & $\mathrm{Y}$ & $\mathrm{N}$ & $\mathrm{Y}$ & $\mathrm{N}$ & $\mathrm{Y}$ & $\mathrm{N}$ & $\mathrm{Y}$ \\
\hline SERS 01 & $\mathrm{~N}$ & $\mathrm{~N}$ & $\mathrm{~N}$ & $\mathrm{~N}$ & $\mathrm{~N}$ & $\mathrm{~N}$ & $\mathrm{~N}$ & $\mathrm{~N}$ & $\mathrm{~N}$ & $\mathrm{~N}$ & Y \\
\hline SERS 02 & $\mathrm{Y}$ & $\mathrm{Y}$ & $\mathrm{Y}$ & $\mathrm{Y}$ & $\mathrm{N}$ & $\mathrm{N}$ & Y & $\mathrm{Y}$ & Y & $\mathrm{N}$ & Y \\
\hline TAU 01 & $\mathrm{Y}$ & $\mathrm{N}$ & $\mathrm{N}$ & $\mathrm{N}$ & $\mathrm{N}$ & $\mathrm{N}$ & $\mathrm{N}$ & $\mathrm{N}$ & $\mathrm{Y}$ & $\mathrm{N}$ & Y \\
\hline TAU 02 & $\mathrm{~N}$ & $\mathrm{~N}$ & $\mathrm{Y}$ & $\mathrm{Y}$ & Y & $\mathrm{N}$ & Y & $\mathrm{N}$ & $\mathrm{N}$ & $\mathrm{N}$ & Y \\
\hline TAU 03 & $\mathrm{Y}$ & $\mathrm{N}$ & $Y$ & $\mathrm{~N}$ & $\mathrm{Y}$ & $\mathrm{N}$ & $\mathrm{Y}$ & Y & $\mathrm{N}$ & $\mathrm{N}$ & Y \\
\hline TAU 04 & $\mathrm{~N}$ & $\mathrm{~N}$ & $\mathrm{~N}$ & $\mathrm{~N}$ & $\mathrm{~N}$ & $\mathrm{~N}$ & $\mathrm{~N}$ & $\mathrm{~N}$ & $\mathrm{~N}$ & $\mathrm{~N}$ & Y \\
\hline TAU 06 & $\mathrm{~N}$ & $\mathrm{~N}$ & $\mathrm{~N}$ & $\mathrm{~N}$ & $\mathrm{~N}$ & $\mathrm{~N}$ & $\mathrm{~N}$ & $\mathrm{~N}$ & $\mathrm{~N}$ & $\mathrm{~N}$ & $\mathrm{Y}$ \\
\hline TAU 07 & $\mathrm{Y}$ & $\mathrm{N}$ & $\mathrm{Y}$ & $\mathrm{Y}$ & $\mathrm{Y}$ & $\mathrm{N}$ & Y & $\mathrm{N}$ & $\mathrm{Y}$ & $\mathrm{N}$ & $\mathrm{Y}$ \\
\hline TAU $08^{a}$ & - & - & - & - & - & - & - & - & - & - & - \\
\hline TAU 09 & Y & $\mathrm{N}$ & $\mathrm{Y}$ & Y & $\mathrm{N}$ & $\mathrm{N}$ & $\mathrm{N}$ & $\mathrm{Y}$ & $\mathrm{N}$ & Y & Y \\
\hline W4001 & $\mathrm{N}$ & $\mathrm{N}$ & $\mathrm{Y}$ & $\mathrm{N}$ & $\mathrm{N}$ & $\mathrm{N}$ & $\mathrm{N}$ & $\mathrm{N}$ & $\mathrm{N}$ & $\mathrm{N}$ & Y \\
\hline W40 02 & $\mathrm{~N}$ & $\mathrm{~N}$ & $\mathrm{Y}$ & $\mathrm{N}$ & $\mathrm{N}$ & $\mathrm{N}$ & $\mathrm{N}$ & $\mathrm{N}$ & $Y$ & $\mathrm{~N}$ & Y \\
\hline W40 03 & $\mathrm{~N}$ & $\mathrm{~N}$ & $\mathrm{~N}$ & $\mathrm{~N}$ & $\mathrm{~N}$ & $\mathrm{~N}$ & $\mathrm{~N}$ & $\mathrm{~N}$ & $\mathrm{~N}$ & $\mathrm{~N}$ & Y \\
\hline W40 04 & $\mathrm{~N}$ & $\mathrm{~N}$ & $\mathrm{~N}$ & $\mathrm{~N}$ & $\mathrm{~N}$ & $\mathrm{~N}$ & $\mathrm{~N}$ & $\mathrm{~N}$ & $\mathrm{~N}$ & $\mathrm{~N}$ & $\mathrm{Y}$ \\
\hline W40 05 & $\mathrm{~N}$ & $\mathrm{~N}$ & $\mathrm{~N}$ & $\mathrm{~N}$ & $\mathrm{~N}$ & $\mathrm{~N}$ & $\mathrm{~N}$ & $\mathrm{~N}$ & $\mathrm{~N}$ & $\mathrm{~N}$ & $\mathrm{Y}$ \\
\hline W40 06 & $\mathrm{~N}$ & $\mathrm{~N}$ & $\mathrm{~N}$ & $\mathrm{~N}$ & $\mathrm{~N}$ & $\mathrm{~N}$ & $\mathrm{~N}$ & $\mathrm{~N}$ & $\mathrm{~N}$ & $\mathrm{~N}$ & $\mathbf{N}^{b}$ \\
\hline W40 07 & $\mathrm{~N}$ & $\mathrm{~N}$ & $\mathrm{~N}$ & $\mathrm{~N}$ & $\mathrm{~N}$ & $\mathrm{~N}$ & $\mathrm{~N}$ & $\mathrm{~N}$ & $\mathrm{~N}$ & $\mathrm{~N}$ & $\mathrm{Y}$ \\
\hline Total & $27 / 48$ & $14 / 48$ & $30 / 48$ & $19 / 48$ & $18 / 48$ & $0 / 48$ & $12 / 48$ & $14 / 48$ & $24 / 48$ & $7 / 48$ & $42 / 48$ \\
\hline
\end{tabular}

Notes. ${ }^{(a)}$ Source not observed with PACS due to end of Herschel mission. ${ }^{(b)}$ In absorption due to contamination of the off-position. ${ }^{(c)}$ The CO $J=31-30$ transition at $84.41 \mu \mathrm{m}$ and the $\mathrm{OH} 84.42 \mu \mathrm{m}$ transition are blended.

the central spectra of all sources for ${ }^{13} \mathrm{CO}, \mathrm{C}^{18} \mathrm{O}, \mathrm{HCO}^{+}$and $\mathrm{H}^{13} \mathrm{CO}^{+}$in order to determine line-widths and central velocities. The $v_{\mathrm{LSR}}$ for each source is defined as the peak position of the fit to the $\mathrm{C}^{18} \mathrm{O} J=3-2$ central spectrum. For the four sources where this is not detected, three (SCO 01, TAU 03, TAU 07) were also observed and detected in ${ }^{13} \mathrm{CO} J=3-2$, so the fit to this line is used instead. For the remaining source (TAU 08) we use the velocity derived by Caselli et al. (2002) from $\mathrm{N}_{2} \mathrm{H}^{+} J=1-0$ observations. The integrated intensity of the lines is measured over a window of \pm 3 FWHM. 
J. C. Mottram et al.: Outflows, infall and evolution of a sample of embedded low-mass protostars

Table A.4. continued.

\begin{tabular}{|c|c|c|c|c|c|c|c|c|c|c|c|c|c|}
\hline Name & $16-14$ & $21-20$ & $24-23$ & $\begin{array}{c}\mathrm{CO} \\
29-28\end{array}$ & $31-30^{c}$ & $32-31$ & $33-32$ & $79.18 \mu \mathrm{m}$ & $79.12 \mu \mathrm{m}$ & $163.40 \mu \mathrm{m}$ & $\begin{array}{l}\mathrm{DH} \\
163.12 \mu \mathrm{m}\end{array}$ & $84.60 \mu \mathrm{m}$ & $84.42 \mu \mathrm{m}^{c}$ \\
\hline AQU 01 & $\mathrm{Y}$ & $\mathrm{Y}$ & $\mathrm{Y}$ & $\mathrm{N}$ & $\mathrm{N}$ & $\mathrm{N}$ & $\mathrm{N}$ & $\mathrm{N}$ & $\mathrm{N}$ & $\mathrm{N}$ & $\mathrm{N}$ & $\mathrm{N}$ & $\mathrm{N}$ \\
\hline AQU 02 & $\mathrm{Y}$ & Y & Y & Y & Y & $\mathrm{N}$ & $\mathrm{N}$ & $\mathrm{N}$ & $\mathrm{N}$ & $\mathrm{N}$ & $\mathrm{N}$ & Y & Y \\
\hline AQU 03 & $\mathrm{~N}$ & $\mathrm{~N}$ & $\mathrm{~N}$ & $\mathrm{~N}$ & $\mathrm{~N}$ & $\mathrm{~N}$ & $\mathrm{~N}$ & $\mathrm{~N}$ & $\mathrm{~N}$ & $\mathrm{~N}$ & $\mathrm{~N}$ & $\mathrm{~N}$ & $\mathrm{~N}$ \\
\hline AQU 04 & $\mathrm{~N}$ & $\mathrm{~N}$ & $\mathrm{~N}$ & $\mathrm{~N}$ & $\mathrm{~N}$ & $\mathrm{~N}$ & $\mathrm{~N}$ & $\mathrm{~N}$ & $\mathrm{~N}$ & $\mathrm{~N}$ & $\mathrm{~N}$ & $\mathrm{~N}$ & $\mathrm{~N}$ \\
\hline AQU 05 & $\mathrm{Y}$ & $\mathrm{Y}$ & $\mathrm{N}$ & $\mathrm{N}$ & $\mathrm{N}$ & $\mathrm{N}$ & $\mathrm{N}$ & $\mathrm{N}$ & $\mathrm{N}$ & $\mathrm{N}$ & $\mathrm{N}$ & $\mathrm{N}$ & $\mathrm{N}$ \\
\hline AQU 06 & $\mathrm{~N}$ & $\mathrm{~N}$ & $\mathrm{~N}$ & $\mathrm{~N}$ & $\mathrm{~N}$ & $\mathrm{~N}$ & $\mathrm{~N}$ & $\mathrm{~N}$ & $\mathrm{~N}$ & $\mathrm{~N}$ & $\mathrm{~N}$ & $\mathrm{~N}$ & $\mathrm{~N}$ \\
\hline CHA 01 & $\mathrm{Y}$ & $\mathrm{Y}$ & $\mathrm{Y}$ & $\mathrm{Y}$ & $\mathrm{Y}$ & $\mathrm{N}$ & $\mathrm{N}$ & $\mathrm{Y}$ & $\mathrm{Y}$ & $\mathrm{Y}$ & $\mathrm{Y}$ & $\mathrm{Y}$ & $\mathrm{Y}$ \\
\hline CHA 02 & Y & $\mathrm{Y}$ & $\mathrm{N}$ & $\mathrm{N}$ & $\mathrm{Y}$ & $\mathrm{N}$ & $\mathrm{N}$ & Y & Y & $\mathrm{N}$ & $\mathrm{N}$ & Y & Y \\
\hline CRA 01 & $\mathrm{Y}$ & $\mathrm{Y}$ & $\mathrm{Y}$ & $\mathrm{Y}$ & $\mathrm{Y}$ & $\mathrm{N}$ & $\mathrm{N}$ & $\mathrm{Y}$ & $\mathrm{Y}$ & Y & Y & $Y$ & $\mathrm{Y}$ \\
\hline ОРН 01 & $\mathrm{Y}$ & $\mathrm{N}$ & $\mathrm{N}$ & $\mathrm{N}$ & $\mathrm{N}$ & $\mathrm{N}$ & $\mathrm{N}$ & $\mathrm{N}$ & $\mathrm{N}$ & $\mathrm{N}$ & $\mathrm{N}$ & $\mathrm{N}$ & $\mathrm{N}$ \\
\hline ОРН 02 & $\mathrm{Y}$ & $\mathrm{Y}$ & $\mathrm{Y}$ & $\mathrm{Y}$ & $\mathrm{Y}$ & $\mathrm{Y}$ & $\mathrm{N}$ & $\mathrm{Y}$ & $\mathrm{Y}$ & $\mathrm{Y}$ & $\mathrm{Y}$ & $\mathrm{Y}$ & $\mathrm{Y}$ \\
\hline PER 01 & $\mathrm{Y}$ & $\mathrm{Y}$ & $\mathrm{Y}$ & $Y$ & $Y$ & $\mathrm{Y}$ & Y & $Y$ & $Y$ & $\mathrm{Y}$ & $\mathrm{Y}$ & $Y$ & $\mathrm{Y}$ \\
\hline PER 02 & $\mathrm{Y}$ & $\mathrm{Y}$ & $\mathrm{Y}$ & $\mathrm{Y}$ & $\mathrm{Y}$ & $\mathrm{Y}$ & $\mathrm{Y}$ & $\mathrm{Y}$ & $\mathrm{Y}$ & Y & $\mathrm{Y}$ & $\mathrm{Y}$ & $\mathrm{Y}$ \\
\hline PER 04 & $\mathrm{~N}$ & $\mathrm{~N}$ & $\mathrm{~N}$ & $\mathrm{~N}$ & $\mathrm{~N}$ & $\mathrm{~N}$ & $\mathrm{~N}$ & $\mathrm{~N}$ & $\mathrm{~N}$ & $\mathrm{~N}$ & $\mathrm{~N}$ & $\mathrm{~N}$ & $\mathrm{~N}$ \\
\hline PER 05 & $\mathrm{Y}$ & $\mathrm{Y}$ & $\mathrm{Y}$ & $\mathrm{Y}$ & $\mathrm{Y}$ & $\mathrm{N}$ & $\mathrm{Y}$ & $\mathrm{Y}$ & $\mathrm{Y}$ & $\mathrm{N}$ & $\mathrm{N}$ & $\mathrm{Y}$ & $\mathrm{Y}$ \\
\hline PER 06 & $\mathrm{Y}$ & $\mathrm{Y}$ & $\mathrm{Y}$ & $\mathrm{N}$ & Y & $\mathrm{Y}$ & $\mathrm{N}$ & $\mathrm{Y}$ & $\mathrm{Y}$ & Y & $\mathrm{Y}$ & $\mathrm{Y}$ & $\mathrm{Y}$ \\
\hline PER 07 & $\mathrm{~N}$ & $\mathrm{~N}$ & $\mathrm{~N}$ & $\mathrm{~N}$ & $\mathrm{~N}$ & $\mathrm{~N}$ & $\mathrm{~N}$ & $\mathrm{~N}$ & $\mathrm{~N}$ & $\mathrm{~N}$ & $\mathrm{~N}$ & $\mathrm{~N}$ & $\mathrm{~N}$ \\
\hline PER 08 & Y & Y & Y & Y & Y & $\mathrm{Y}$ & $\mathrm{N}$ & Y & Y & Y & Y & Y & Y \\
\hline PER 09 & $\mathrm{Y}$ & $\mathrm{Y}$ & $\mathrm{Y}$ & $\mathrm{Y}$ & Y & $\mathrm{Y}$ & $\mathrm{Y}$ & $\mathrm{Y}$ & $\mathrm{Y}$ & Y & Y & Y & $Y$ \\
\hline PER 10 & $\mathrm{Y}$ & Y & Y & Y & Y & $\mathrm{N}$ & $\mathrm{N}$ & $\mathrm{Y}$ & Y & Y & Y & Y & Y \\
\hline PER 12 & $\mathrm{~N}$ & $\mathrm{~N}$ & $\mathrm{~N}$ & $\mathrm{~N}$ & $\mathrm{~N}$ & $\mathrm{~N}$ & $\mathrm{~N}$ & $\mathrm{~N}$ & $\mathrm{~N}$ & $\mathrm{~N}$ & $\mathrm{~N}$ & $\mathrm{~N}$ & $\mathrm{~N}$ \\
\hline PER 13 & $\mathrm{~N}$ & $\mathrm{~N}$ & $\mathrm{~N}$ & $\mathrm{~N}$ & $\mathrm{~N}$ & $\mathrm{~N}$ & $\mathrm{~N}$ & $\mathrm{~N}$ & $\mathrm{~N}$ & $\mathrm{~N}$ & $\mathrm{~N}$ & $\mathrm{~N}$ & $\mathrm{~N}$ \\
\hline PER 14 & $\mathrm{Y}$ & $\mathrm{Y}$ & $\mathrm{Y}$ & $\mathrm{Y}$ & $\mathrm{Y}$ & $\mathrm{Y}$ & $\mathrm{Y}$ & $\mathrm{Y}$ & $\mathrm{Y}$ & $\mathrm{Y}$ & $\mathrm{Y}$ & $\mathrm{Y}$ & $\mathrm{Y}$ \\
\hline PER 15 & $\mathrm{Y}$ & $\mathrm{Y}$ & $\mathrm{Y}$ & $\mathrm{N}$ & $\mathrm{Y}$ & $\mathrm{N}$ & $\mathrm{N}$ & $\mathrm{N}$ & $\mathrm{N}$ & $\mathrm{Y}$ & $\mathrm{N}$ & $\mathrm{Y}$ & $\mathrm{Y}$ \\
\hline PER 16 & $\mathrm{Y}$ & $\mathrm{Y}$ & $\mathrm{N}$ & $\mathrm{N}$ & $\mathrm{N}$ & $\mathrm{N}$ & $\mathrm{N}$ & $\mathrm{N}$ & $\mathrm{N}$ & $\mathrm{N}$ & $\mathrm{N}$ & $\mathrm{N}$ & $\mathrm{N}$ \\
\hline PER 17 & $\mathrm{~N}$ & $\mathrm{~N}$ & $\mathrm{~N}$ & $\mathrm{~N}$ & $\mathrm{~N}$ & $\mathrm{~N}$ & $\mathrm{~N}$ & $\mathrm{~N}$ & $\mathrm{~N}$ & $\mathrm{~N}$ & $\mathrm{~N}$ & $\mathrm{~N}$ & $\mathrm{~N}$ \\
\hline PER 18 & $\mathrm{Y}$ & $\mathrm{Y}$ & $\mathrm{Y}$ & $\mathrm{N}$ & $\mathrm{N}$ & $\mathrm{N}$ & $\mathrm{N}$ & $\mathrm{N}$ & $\mathrm{N}$ & Y & Y & $\mathrm{N}$ & $\mathrm{N}$ \\
\hline PER 19 & $\mathrm{Y}$ & $\mathrm{Y}$ & $\mathrm{Y}$ & $\mathrm{N}$ & Y & $\mathrm{N}$ & $\mathrm{N}$ & $\mathrm{Y}$ & $\mathrm{Y}$ & $\mathrm{Y}$ & $\mathrm{Y}$ & Y & Y \\
\hline PER 20 & Y & Y & Y & $\mathrm{N}$ & Y & $\mathrm{N}$ & $\mathrm{N}$ & $\mathrm{Y}$ & $\mathrm{Y}$ & Y & Y & Y & Y \\
\hline PER 21 & $\mathrm{Y}$ & $\mathrm{Y}$ & $\mathrm{Y}$ & Y & $\mathrm{Y}$ & $\mathrm{N}$ & $\mathrm{N}$ & $\mathrm{Y}$ & $\mathrm{Y}$ & $\mathrm{Y}$ & $\mathrm{N}$ & $\mathrm{Y}$ & $\mathrm{Y}$ \\
\hline PER 22 & $\mathrm{Y}$ & $\mathrm{Y}$ & $\mathrm{Y}$ & $\mathrm{N}$ & $\mathrm{Y}$ & $\mathrm{N}$ & $\mathrm{N}$ & $\mathrm{Y}$ & Y & $\mathrm{N}$ & $\mathrm{Y}$ & $\mathrm{Y}$ & $\mathrm{Y}$ \\
\hline SCO 01 & $\mathrm{Y}$ & $\mathrm{Y}$ & $\mathrm{Y}$ & $\mathrm{Y}$ & $\mathrm{Y}$ & $\mathrm{N}$ & $\mathrm{N}$ & $\mathrm{Y}$ & $\mathrm{Y}$ & $\mathrm{Y}$ & $\mathrm{Y}$ & $Y$ & $\mathrm{Y}$ \\
\hline SERS 01 & $\mathrm{~N}$ & $\mathrm{~N}$ & $\mathrm{~N}$ & $\mathrm{~N}$ & $\mathrm{~N}$ & $\mathrm{~N}$ & $\mathrm{~N}$ & $\mathrm{~N}$ & $\mathrm{~N}$ & $\mathrm{~N}$ & $\mathrm{~N}$ & $\mathrm{~N}$ & $\mathrm{~N}$ \\
\hline SERS 02 & Y & $\mathrm{Y}$ & Y & $\mathrm{Y}$ & Y & $\mathrm{N}$ & $\mathrm{N}$ & $\mathrm{N}$ & $\mathrm{N}$ & $\mathrm{N}$ & $\mathrm{N}$ & $\mathrm{Y}$ & Y \\
\hline TAU 01 & $\mathrm{Y}$ & $\mathrm{Y}$ & $\mathrm{N}$ & $\mathrm{N}$ & $\mathrm{Y}$ & $\mathrm{N}$ & $\mathrm{N}$ & Y & $\mathrm{Y}$ & $\mathrm{N}$ & $\mathrm{N}$ & $\mathrm{Y}$ & $\mathrm{Y}$ \\
\hline TAU 02 & $\mathrm{Y}$ & Y & Y & $\mathrm{N}$ & Y & $\mathrm{N}$ & $\mathrm{N}$ & Y & $\mathrm{Y}$ & Y & Y & $Y$ & Y \\
\hline TAU 03 & $\mathrm{~N}$ & $\mathrm{Y}$ & $\mathrm{Y}$ & $\mathrm{N}$ & $\mathrm{N}$ & $\mathrm{N}$ & $\mathrm{N}$ & $\mathrm{Y}$ & $\mathrm{Y}$ & $\mathrm{N}$ & $\mathrm{N}$ & $Y$ & $\mathrm{~N}$ \\
\hline TAU 04 & Y & Y & $\mathrm{N}$ & Y & Y & $\mathrm{N}$ & $\mathrm{N}$ & $\mathrm{Y}$ & $\mathrm{Y}$ & $\mathrm{N}$ & $\mathrm{N}$ & $\mathrm{Y}$ & Y \\
\hline TAU 06 & $\mathrm{~N}$ & $\mathrm{~N}$ & $\mathrm{~N}$ & $\mathrm{~N}$ & $\mathrm{~N}$ & $\mathrm{~N}$ & $\mathrm{~N}$ & $\mathrm{~N}$ & $\mathrm{~N}$ & $\mathrm{~N}$ & $\mathrm{~N}$ & $\mathrm{~N}$ & $\mathrm{~N}$ \\
\hline TAU 07 & $\mathrm{Y}$ & Y & $\mathrm{Y}$ & $\mathrm{Y}$ & Y & $\mathrm{N}$ & $\mathrm{N}$ & $\mathrm{Y}$ & $\mathrm{Y}$ & Y & $\mathrm{Y}$ & $\mathrm{Y}$ & $\mathrm{Y}$ \\
\hline TAU $08^{a}$ & - & - & - & - & - & - & - & - & - & - & - & - & - \\
\hline TAU 09 & $\mathrm{Y}$ & $\mathrm{Y}$ & $\mathrm{Y}$ & $\mathrm{Y}$ & $\mathrm{Y}$ & $\mathrm{N}$ & $\mathrm{N}$ & $\mathrm{N}$ & $\mathrm{N}$ & $\mathrm{Y}$ & $\mathrm{N}$ & $\mathrm{Y}$ & $\mathrm{Y}$ \\
\hline W4001 & Y & $\mathrm{Y}$ & $\mathrm{N}$ & $\mathrm{N}$ & $\mathrm{N}$ & $\mathrm{N}$ & $\mathrm{N}$ & $\mathrm{N}$ & $\mathrm{N}$ & $\mathrm{N}$ & $\mathrm{N}$ & $\mathrm{N}$ & $\mathrm{N}$ \\
\hline W40 02 & $\mathrm{Y}$ & $\mathrm{Y}$ & $\mathrm{Y}$ & $\mathrm{N}$ & Y & $\mathrm{N}$ & $\mathrm{N}$ & $\mathrm{N}$ & $\mathrm{N}$ & Y & $\mathrm{Y}$ & $\mathrm{Y}$ & $\mathrm{Y}$ \\
\hline W40 03 & $\mathrm{~N}$ & $\mathrm{~N}$ & $\mathrm{~N}$ & $\mathrm{~N}$ & $\mathrm{~N}$ & $\mathrm{~N}$ & $\mathrm{~N}$ & $\mathrm{~N}$ & $\mathrm{~N}$ & $\mathrm{~N}$ & $\mathrm{~N}$ & $\mathrm{~N}$ & $\mathrm{~N}$ \\
\hline W40 04 & $\mathrm{~N}$ & $\mathrm{~N}$ & $\mathrm{~N}$ & $\mathrm{~N}$ & $\mathrm{~N}$ & $\mathrm{~N}$ & $\mathrm{~N}$ & $\mathrm{~N}$ & $\mathrm{~N}$ & $\mathrm{~N}$ & $\mathrm{~N}$ & $\mathrm{~N}$ & $\mathrm{~N}$ \\
\hline W40 05 & $\mathrm{~N}$ & $\mathrm{~N}$ & $\mathrm{~N}$ & $\mathrm{~N}$ & $\mathrm{~N}$ & $\mathrm{~N}$ & $\mathrm{~N}$ & $\mathrm{~N}$ & $\mathrm{~N}$ & $\mathrm{~N}$ & $\mathrm{~N}$ & $\mathrm{~N}$ & $\mathrm{~N}$ \\
\hline W4006 & $\mathrm{N}$ & $\mathrm{N}$ & $\mathrm{N}$ & $\mathrm{N}$ & $\mathrm{N}$ & $\mathrm{N}$ & $\mathrm{N}$ & $\mathrm{N}$ & $\mathrm{N}$ & $\mathrm{N}$ & $\mathrm{N}$ & $\mathrm{N}$ & $\mathrm{N}$ \\
\hline W40 07 & $\mathrm{~N}$ & $\mathrm{~N}$ & $\mathrm{~N}$ & $\mathrm{~N}$ & $\mathrm{~N}$ & $\mathrm{~N}$ & $\mathrm{~N}$ & $\mathrm{~N}$ & $\mathrm{~N}$ & $\mathrm{~N}$ & $\mathrm{~N}$ & $\mathrm{~N}$ & $\mathrm{~N}$ \\
\hline Total & $32 / 48$ & $32 / 48$ & $26 / 48$ & $17 / 48$ & $26 / 48$ & $7 / 48$ & $5 / 48$ & $22 / 48$ & $22 / 48$ & $20 / 48$ & $18 / 48$ & $27 / 48$ & $26 / 48$ \\
\hline
\end{tabular}

Table A.5. Correction factors for line-of-sight outflow inclination.

\begin{tabular}{lllll}
\hline \hline$i$ & $10^{\circ}$ & $30^{\circ}$ & $50^{\circ}$ & $70^{\circ}$ \\
\hline$c_{\mathrm{f}}$ & 1.2 & 2.8 & 4.4 & 7.1 \\
\hline
\end{tabular}

For the optically thick $\mathrm{HCO}^{+}$and ${ }^{13} \mathrm{CO}$, we also quantify any blue/red asymmetry by calculating $\delta v$ as defined by
Mardones et al. (1997) using Eq. (11). For most sources, we use the peak of the Gaussian fit to the line, but in the case of five sources (AQU 02, PER 04, SERS 01, SERS 02 and TAU 09), the position of the maximum intensity is used in this calculation for $\mathrm{HCO}^{+}$because strong self-absorption or a broad, fainter second component skews the Gaussian fit away from the maximum intensity. 
Table A.6. Outflow properties.

\begin{tabular}{|c|c|c|c|c|c|c|c|c|c|c|c|c|}
\hline Name & $\begin{array}{c}i \\
\left({ }^{\circ}\right) \\
\end{array}$ & Lobe & $\begin{array}{c}v_{\text {in }}{ }^{a} \\
\left(\mathrm{~km} \mathrm{~s}^{-1}\right) \\
\end{array}$ & $\begin{array}{c}\begin{array}{c}v_{\text {out }}{ }^{a} \\
\left(\mathrm{~km} \mathrm{~s}^{-1}\right)\end{array} \\
\end{array}$ & $\begin{array}{l}\left|v_{\max }\right|^{a} \\
\left(\mathrm{~km} \mathrm{~s}^{-1}\right)\end{array}$ & $\begin{array}{c}M_{\text {out }^{a}} \\
\left(M_{\odot}\right)\end{array}$ & $\begin{array}{c}P_{\text {out }}{ }^{a} \\
\left(M_{\odot} \mathrm{km} \mathrm{s}^{-1}\right) \\
\end{array}$ & $\begin{array}{l}E_{\text {out }}{ }^{a} \\
\text { (erg) }\end{array}$ & $\begin{array}{c}R_{\mathrm{CO}}{ }^{a} \\
\left(10^{3} \mathrm{AU}\right) \\
\end{array}$ & $\begin{array}{c}t_{\mathrm{dyn}}{ }^{a} \\
\left(10^{3} \mathrm{yr}\right) \\
\end{array}$ & $\begin{array}{c}F_{\mathrm{CO}}^{b} \\
\left(M_{\odot} \mathrm{yr}^{-1} \mathrm{~km} \mathrm{~s}^{-1}\right) \\
\end{array}$ & $\begin{array}{c}\dot{M}_{\text {out }}{ }^{b} \\
\left(M_{\odot} \mathrm{yr}^{-1}\right)\end{array}$ \\
\hline AQU 01 & 70 & $\begin{array}{l}\mathrm{R} \\
\mathrm{B}\end{array}$ & $\begin{array}{r}11.0 \\
3.0\end{array}$ & $\begin{array}{r}33.5 \\
-30.0 \\
\end{array}$ & $\begin{array}{l}26.1 \\
37.4 \\
\end{array}$ & $\begin{array}{l}1.6 \times 10^{-02} \\
2.2 \times 10^{-02} \\
\end{array}$ & $\begin{array}{l}.5 \times 10^{-01} \\
2.2 \times 10^{-01} \\
\end{array}$ & $\begin{array}{l}1.8 \times 10^{+43} \\
3.3 \times 10^{+43} \\
\end{array}$ & $\begin{array}{l}15.0 \\
28.1\end{array}$ & $\begin{array}{l}2.7 \\
3.6 \\
\end{array}$ & $\begin{array}{l}3.9 \times 10^{-04} \\
4.5 \times 10^{-04} \\
\end{array}$ & $\begin{array}{l}4.2 \times 10^{-05} \\
4.3 \times 10^{-05} \\
\end{array}$ \\
\hline AQU 02 & 30 & $\begin{array}{l}\mathrm{R} \\
\mathrm{B}\end{array}$ & $\begin{array}{r}10.5 \\
4.5 \\
\end{array}$ & $\begin{array}{r}25.0 \\
-8.5 \\
\end{array}$ & & $\begin{array}{l}7.4 \times 10^{-03} \\
4.8 \times 10^{-03} \\
\end{array}$ & $\begin{array}{l}4.2 \times 10^{-02} \\
3.1 \times 10^{-02} \\
\end{array}$ & $\begin{array}{l}3.1 \times 10^{+42} \\
2.6 \times 10^{+42}\end{array}$ & $\begin{array}{r}16.4 \\
5.5 \\
\end{array}$ & $\begin{array}{l}4.4 \\
1.6 \\
\end{array}$ & $\begin{array}{l}2.7 \times 10^{-05} \\
5.4 \times 10^{-05} \\
\end{array}$ & $\begin{array}{l}4.6 \times 10^{-06} \\
8.2 \times 10^{-06} \\
\end{array}$ \\
\hline AQU 03 & $-^{c}$ & - & - & - & - & - & - & - & - & - & - & - \\
\hline AQU 04 & 70 & $\begin{array}{l}\mathrm{R} \\
\mathrm{B}\end{array}$ & $\begin{array}{r}11.0 \\
2.5 \\
\end{array}$ & $\begin{array}{r}17.5 \\
-1.0 \\
\end{array}$ & $\begin{array}{c}9.9 \\
8.6 \\
\end{array}$ & $\begin{array}{l}7.7 \times 10^{-03} \\
8.7 \times 10^{-04}\end{array}$ & $\begin{array}{l}3.7 \times 10^{-02} \\
5.4 \times 10^{-03}\end{array}$ & $\begin{array}{l}1.9 \times 10^{+42} \\
3.4 \times 10^{+41}\end{array}$ & $\begin{array}{l}29.2 \\
10.0\end{array}$ & $\begin{array}{r}14.0 \\
5.5 \\
\end{array}$ & $\begin{array}{l}1.9 \times 10^{-05} \\
6.9 \times 10^{-06} \\
\end{array}$ & $\begin{array}{l}3.9 \times 10^{-06} \\
1.1 \times 10^{-06} \\
\end{array}$ \\
\hline AQU 05 & 30 & $\begin{array}{l}\mathrm{R} \\
\mathrm{B} \\
\end{array}$ & $\begin{array}{r}11.0 \\
2.5 \\
\end{array}$ & $\begin{array}{r}18.5 \\
-4.0 \\
\end{array}$ & & $\begin{array}{l}1.9 \times 10^{-02} \\
1.5 \times 10^{-03}\end{array}$ & $\begin{array}{l}9.8 \times 10^{-02} \\
1.2 \times 10^{-02} \\
\end{array}$ & $\begin{array}{l}5.5 \times 10^{+42} \\
9.8 \times 10^{+41} \\
\end{array}$ & $\begin{array}{l}15.1 \\
16.4 \\
\end{array}$ & $\begin{array}{l}6.4 \\
6.8 \\
\end{array}$ & $\begin{array}{l}4.3 \times 10^{-05} \\
4.8 \times 10^{-06} \\
\end{array}$ & $\begin{array}{l}8.2 \times 10^{-06} \\
6.1 \times 10^{-07} \\
\end{array}$ \\
\hline AQU 06 & 10 & $\begin{array}{l}\mathrm{R} \\
\mathrm{B}\end{array}$ & & $\begin{array}{r}18.5 \\
-3.5 \\
\end{array}$ & & $\begin{array}{l}2.7 \times 10^{-03} \\
1.1 \times 10^{-03} \\
\end{array}$ & & $\begin{array}{l}6.5 \times 10^{+41} \\
7.7 \times 10^{+41}\end{array}$ & & & $\begin{array}{l}6.0 \times 10^{-06} \\
5.0 \times 10^{-06}\end{array}$ & \\
\hline CHA 01 & 30 & $\begin{array}{l}\mathrm{R} \\
\mathrm{B}\end{array}$ & & $\begin{array}{r}12.0 \\
2.0 \\
\end{array}$ & & & $\begin{array}{l}3.7 \times 10^{-03} \\
2.0 \times 10^{-04}\end{array}$ & $\begin{array}{l}1.5 \times 10^{+41} \\
4.8 \times 10^{+39} \\
\end{array}$ & $\begin{array}{r}10.7 \\
4.8 \\
\end{array}$ & $\begin{array}{l}7.1 \\
7.8 \\
\end{array}$ & $\begin{array}{l}1.4 \times 10^{-06} \\
7.3 \times 10^{-08} \\
\end{array}$ & $\begin{array}{l}3.8 \times 10^{-07} \\
3.2 \times 10^{-08} \\
\end{array}$ \\
\hline CHA 02 & $-^{c}$ & - & - & - & - & - & - & - & - & - & - & - \\
\hline CRA 01 & 50 & $\begin{array}{l}\mathrm{R} \\
\mathrm{B}\end{array}$ & & $\begin{array}{r}22.8 \\
-16.7 \\
\end{array}$ & & & & & & & & \\
\hline OPH 01 & $-^{c}$ & - & - & - & - & - & - & - & - & - & - & - \\
\hline OPH 02 & 10 & $\begin{array}{l}\mathrm{R} \\
\mathrm{B} \\
\end{array}$ & & & & & & & $\begin{array}{r}9.2 \\
11.8 \\
\end{array}$ & $\begin{array}{l}7.0 \\
6.8 \\
\end{array}$ & & \\
\hline PER 01 & 70 & $\begin{array}{l}\mathrm{R} \\
\mathrm{B}\end{array}$ & & $\begin{array}{r}49.2 \\
-36.3 \\
\end{array}$ & & & & $\begin{array}{l}3.2 \times 10^{+43} \\
2.9 \times 10^{+43} \\
\end{array}$ & & & & $\begin{array}{l}3.9 \times 10^{-05} \\
4.2 \times 10^{-05} \\
\end{array}$ \\
\hline PER 02 & $-^{d}$ & - & - & - & - & - & - & - & - & - & - & - \\
\hline PER 04 & 50 & $\begin{array}{l}\mathrm{R} \\
\mathrm{B}\end{array}$ & & & & & & & & $\begin{array}{c}17.6 \\
9.6 \\
\end{array}$ & & \\
\hline PER 05 & 10 & $\begin{array}{l}\mathrm{R} \\
\mathrm{B}\end{array}$ & $\begin{array}{r}12.7 \\
3.2 \\
\end{array}$ & $\begin{array}{r}17.7 \\
-1.3 \\
\end{array}$ & & & & & & $\begin{array}{l}6.3 \\
7.7 \\
\end{array}$ & $\begin{array}{l}3.5 \times 10^{-07} \\
2.9 \times 10^{-07}\end{array}$ & $\begin{array}{l}4.8 \times 10^{-08} \\
5.4 \times 10^{-08}\end{array}$ \\
\hline PER 06 & $-^{d}$ & - & - & - & - & - & - & - & - & - & - & - \\
\hline PER 07 & 70 & $\begin{array}{l}\mathrm{R} \\
\mathrm{B}\end{array}$ & $\begin{array}{r}11.2 \\
2.2 \\
\end{array}$ & $\begin{array}{r}20.7 \\
-5.3 \\
\end{array}$ & & & & & & $\begin{array}{r}10.4 \\
6.6 \\
\end{array}$ & & \\
\hline PER 08 & 10 & $\begin{array}{l}\mathrm{R} \\
\mathrm{B} \\
\end{array}$ & & $\begin{array}{r}21.5 \\
-14.5 \\
\end{array}$ & & & & & & & & \\
\hline PER 09 & 70 & $\begin{array}{l}\mathrm{R} \\
\mathrm{B}\end{array}$ & $\begin{array}{r}13.0 \\
0.5\end{array}$ & $\begin{array}{r}25.0 \\
-16.0 \\
\end{array}$ & & & & & & $\begin{array}{l}4.0 \\
2.8\end{array}$ & $\begin{array}{l}8.8 \times 10^{-06} \\
2.3 \times 10^{-05} \\
\end{array}$ & $\begin{array}{l}9.6 \times 10^{-07} \\
1.9 \times 10^{-06}\end{array}$ \\
\hline PER 10 & 50 & $\begin{array}{l}\mathrm{R} \\
\mathrm{B} \\
\end{array}$ & $\begin{array}{r}13.2 \\
2.2 \\
\end{array}$ & $\begin{array}{r}32.2 \\
-14.8 \\
\end{array}$ & & & & & & $\begin{array}{l}2.9 \\
5.0 \\
\end{array}$ & $\begin{array}{l}1.5 \times 10^{-04} \\
5.3 \times 10^{-05} \\
\end{array}$ & $\begin{array}{l}1.9 \times 10^{-05} \\
5.4 \times 10^{-06} \\
\end{array}$ \\
\hline PER 12 & $-^{c}$ & - & - & - & - & - & - & - & - & - & - & - \\
\hline PER 13 & 70 & $\begin{array}{l}\mathrm{R} \\
\mathrm{B} \\
\end{array}$ & $\begin{array}{r}10.7 \\
5.7 \\
\end{array}$ & $\begin{array}{r}22.2 \\
-4.3 \\
\end{array}$ & & & & & $\begin{array}{l}14.2 \\
27.0 \\
\end{array}$ & $\begin{array}{r}4.7 \\
10.4 \\
\end{array}$ & $\begin{array}{l}2.9 \times 10^{-05} \\
5.4 \times 10^{-06} \\
\end{array}$ & $\begin{array}{l}6.1 \times 10^{-06} \\
1.2 \times 10^{-06} \\
\end{array}$ \\
\hline PER 14 & 50 & $\begin{array}{l}\mathrm{R} \\
\mathrm{B}\end{array}$ & & & & & & & $\begin{array}{r}4.0 \\
27.9 \\
\end{array}$ & $\begin{array}{l}1.4 \\
8.7 \\
\end{array}$ & & $\begin{array}{l}9.7 \times 10^{-07} \\
2.7 \times 10^{-06}\end{array}$ \\
\hline PER 15 & 70 & $\begin{array}{l}\mathrm{R} \\
\mathrm{B} \\
\end{array}$ & $\begin{array}{c}9.7 \\
1.2 \\
\end{array}$ & $\begin{array}{r}28.2 \\
-25.3 \\
\end{array}$ & & & & & & $\begin{array}{l}3.9 \\
5.4\end{array}$ & $\begin{array}{l}1.2 \times 10^{-04} \\
6.9 \times 10^{-05} \\
\end{array}$ & $\begin{array}{l}1.8 \times 10^{-05} \\
6.4 \times 10^{-06} \\
\end{array}$ \\
\hline PER 16 & 70 & $\begin{array}{l}\mathrm{R} \\
\mathrm{B}\end{array}$ & & $\begin{array}{c}23.2 \\
-6.3 \\
\end{array}$ & & & $\begin{array}{l}2.3 \times 10^{-02} \\
1.4 \times 10^{-02}\end{array}$ & $\begin{array}{l}1.7 \times 10^{+42} \\
1.1 \times 10^{+42}\end{array}$ & $\begin{array}{l}17.4 \\
37.0\end{array}$ & $\begin{array}{r}5.1 \\
13.2 \\
\end{array}$ & $\begin{array}{l}3.3 \times 10^{-05} \\
7.8 \times 10^{-06}\end{array}$ & $\begin{array}{l}6.4 \times 10^{-06} \\
1.2 \times 10^{-06}\end{array}$ \\
\hline PER 17 & 50 & $\begin{array}{l}\mathrm{R} \\
\mathrm{B} \\
\end{array}$ & $\begin{array}{r}10.8 \\
2.8 \\
\end{array}$ & $\begin{array}{r}30.3 \\
-20.2 \\
\end{array}$ & & & $\begin{array}{l}3.8 \times 10^{-02} \\
5.2 \times 10^{-02}\end{array}$ & $\begin{array}{l}3.4 \times 10^{+42} \\
4.9 \times 10^{+42}\end{array}$ & $\begin{array}{l}12.8 \\
30.1 \\
\end{array}$ & $\begin{array}{l}2.6 \\
5.3 \\
\end{array}$ & $\begin{array}{l}6.6 \times 10^{-05} \\
4.3 \times 10^{-05} \\
\end{array}$ & $\begin{array}{l}8.9 \times 10^{-06} \\
5.9 \times 10^{-06}\end{array}$ \\
\hline PER 18 & 70 & $\begin{array}{l}\mathrm{R} \\
\mathrm{B}\end{array}$ & $\begin{array}{r}10.8 \\
2.8 \\
\end{array}$ & $\begin{array}{r}32.3 \\
-5.7 \\
\end{array}$ & & & $\begin{array}{l}2.1 \times 10^{-02} \\
3.9 \times 10^{-03}\end{array}$ & $\begin{array}{l}2.7 \times 10^{+42} \\
2.5 \times 10^{+41}\end{array}$ & $\begin{array}{l}32.4 \\
12.7 \\
\end{array}$ & $\begin{array}{l}6.0 \\
4.9 \\
\end{array}$ & $\begin{array}{l}2.5 \times 10^{-05} \\
5.7 \times 10^{-06}\end{array}$ & $\begin{array}{l}2.4 \times 10^{-06} \\
1.0 \times 10^{-06}\end{array}$ \\
\hline PER 19 & 50 & $\begin{array}{l}\mathrm{R} \\
\mathrm{B}\end{array}$ & $\begin{array}{c}9.7 \\
2.7 \\
\end{array}$ & $\begin{array}{r}15.7 \\
-4.3 \\
\end{array}$ & $\begin{array}{r}9.0 \\
11.0 \\
\end{array}$ & $\begin{array}{l}2.4 \times 10^{-03} \\
6.0 \times 10^{-04} \\
\end{array}$ & $\begin{array}{l}9.3 \times 10^{-03} \\
3.2 \times 10^{-03}\end{array}$ & $\begin{array}{l}3.9 \times 10^{+41} \\
1.9 \times 10^{+41}\end{array}$ & $\begin{array}{l}17.4 \\
11.8 \\
\end{array}$ & $\begin{array}{l}9.2 \\
5.1 \\
\end{array}$ & $\begin{array}{l}4.5 \times 10^{-06} \\
2.8 \times 10^{-06} \\
\end{array}$ & $\begin{array}{l}1.2 \times 10^{-06} \\
5.2 \times 10^{-07}\end{array}$ \\
\hline & 50 & $\begin{array}{l}\mathrm{R} \\
\mathrm{B}\end{array}$ & $\begin{array}{r}11.2 \\
6.2\end{array}$ & $\begin{array}{r}50.2 \\
-13.8\end{array}$ & $\begin{array}{l}41.3 \\
22.7\end{array}$ & $\begin{array}{l}5.6 \times 10^{-03} \\
4.7 \times 10^{-03}\end{array}$ & $\begin{array}{l}6.0 \times 10^{-02} \\
3.8 \times 10^{-02}\end{array}$ & $\begin{array}{l}1.2 \times 10^{+43} \\
4.2 \times 10^{+42}\end{array}$ & $\begin{array}{l}11.8 \\
28.1\end{array}$ & $\begin{array}{l}1.4 \\
5.9\end{array}$ & $\begin{array}{l}1.9 \times 10^{-04} \\
2.8 \times 10^{-05}\end{array}$ & $\begin{array}{l}1.8 \times 10^{-05} \\
3.5 \times 10^{-06}\end{array}$ \\
\hline
\end{tabular}

Notes. ${ }^{(a)}$ Measured values, i.e. not corrected for inclination. ${ }^{(b)}$ Corrected for inclination as discussed in detail in van der Marel et al. (2013) and Yildiz et al. (2015). ${ }^{(c)}$ No outflow associated with this source. ${ }^{(d)}$ An outflow is detected but is too contaminated by outflows from others sources for meaningful determinations to be made. See Sect. C for details. 
Table A.6. continued.

\begin{tabular}{|c|c|c|c|c|c|c|c|c|c|c|c|c|}
\hline Name & $\begin{array}{c}i \\
\left({ }^{\circ}\right) \\
\end{array}$ & Lobe & $\begin{array}{c}\begin{array}{c}v_{\text {in }}{ }^{a} \\
\left(\mathrm{~km} \mathrm{~s}^{-1}\right)\end{array} \\
\end{array}$ & $\begin{array}{c}\begin{array}{c}v_{\text {out }}{ }^{a} \\
\left(\mathrm{~km} \mathrm{~s}^{-1}\right)\end{array} \\
\end{array}$ & $\begin{array}{l}\left|v_{\max }\right|^{a} \\
\left(\mathrm{~km} \mathrm{~s}^{-1}\right)\end{array}$ & $\begin{array}{c}M_{\text {out }}{ }^{a} \\
\left(M_{\odot}\right)\end{array}$ & $\begin{array}{c}P_{\text {out }^{a}}{ }^{-1} \\
\left(M_{\odot} \mathrm{km} \mathrm{s}^{-1}\right)\end{array}$ & $\begin{array}{l}E_{\text {out }}{ }^{a} \\
(\mathrm{erg})\end{array}$ & $\begin{array}{c}R_{\mathrm{CO}}{ }^{a} \\
\left(10^{3} \mathrm{AU}\right) \\
\end{array}$ & $\begin{array}{c}t_{\mathrm{dyn}}{ }^{a} \\
\left(10^{3} \mathrm{yr}\right)\end{array}$ & $\begin{array}{c}F_{\mathrm{CO}}^{b} \\
\left(M_{\odot} \mathrm{yr}^{-1} \mathrm{~km} \mathrm{~s}^{-1}\right)\end{array}$ & $\begin{array}{c}\dot{M}_{\text {out }}{ }^{b} \\
\left(M_{\odot} \mathrm{yr}^{-1}\right)\end{array}$ \\
\hline PER 21 & 50 & $\begin{array}{l}\mathrm{R} \\
\mathrm{B}\end{array}$ & $\begin{array}{r}11.2 \\
4.7 \\
\end{array}$ & $\begin{array}{r}28.2 \\
-15.8 \\
\end{array}$ & $\begin{array}{l}19.5 \\
24.5 \\
\end{array}$ & $\begin{array}{l}4.0 \times 10^{-03} \\
2.4 \times 10^{-03} \\
\end{array}$ & $\begin{array}{l}2.5 \times 10^{-02} \\
2.2 \times 10^{-02} \\
\end{array}$ & $\begin{array}{l}2.3 \times 10^{+42} \\
2.6 \times 10^{+42}\end{array}$ & $\begin{array}{l}26.4 \\
10.8 \\
\end{array}$ & $\begin{array}{l}6.4 \\
2.1 \\
\end{array}$ & $\begin{array}{l}1.7 \times 10^{-05} \\
4.6 \times 10^{-05}\end{array}$ & $\begin{array}{l}2.7 \times 10^{-06} \\
5.1 \times 10^{-06} \\
\end{array}$ \\
\hline PER 22 & 30 & $\begin{array}{l}\mathrm{R} \\
\mathrm{B}\end{array}$ & $\begin{array}{r}11.2 \\
6.2 \\
\end{array}$ & $\begin{array}{r}18.2 \\
3.2 \\
\end{array}$ & $\begin{array}{l}8.5 \\
6.5 \\
\end{array}$ & $\begin{array}{l}5.4 \times 10^{-03} \\
9.0 \times 10^{-04}\end{array}$ & $\begin{array}{l}1.2 \times 10^{-02} \\
3.6 \times 10^{-03} \\
\end{array}$ & $\begin{array}{l}3.2 \times 10^{+41} \\
1.5 \times 10^{+41}\end{array}$ & $\begin{array}{l}21.9 \\
10.8\end{array}$ & $\begin{array}{r}12.3 \\
7.8 \\
\end{array}$ & $\begin{array}{l}2.7 \times 10^{-06} \\
1.3 \times 10^{-06} \\
\end{array}$ & $\begin{array}{l}1.2 \times 10^{-06} \\
3.2 \times 10^{-07}\end{array}$ \\
\hline SCO 01 & $-^{c}$ & - & - & - & - & - & - & - & - & - & - & - \\
\hline SERS 01 & 50 & $\begin{array}{l}\mathrm{R} \\
\mathrm{B}\end{array}$ & $\begin{array}{r}11.0 \\
3.5 \\
\end{array}$ & $\begin{array}{r}18.5 \\
-15.0 \\
\end{array}$ & & $\begin{array}{l}1.5 \times 10^{-02} \\
1.6 \times 10^{-02}\end{array}$ & $\begin{array}{l}6.4 \times 10^{-02} \\
1.2 \times 10^{-01} \\
\end{array}$ & $\begin{array}{l}3.1 \times 10^{+42} \\
1.0 \times 10^{+43}\end{array}$ & & $\begin{array}{l}9.1 \\
6.6 \\
\end{array}$ & $\begin{array}{l}3.1 \times 10^{-05} \\
7.8 \times 10^{-05} \\
\end{array}$ & $\begin{array}{l}7.2 \times 10^{-06} \\
1.1 \times 10^{-05}\end{array}$ \\
\hline SERS 02 & 50 & $\begin{array}{l}\mathrm{R} \\
\mathrm{B}\end{array}$ & & $\begin{array}{r}40.5 \\
-18.5 \\
\end{array}$ & & $\begin{array}{l}1.0 \times 10^{-01} \\
3.0 \times 10^{-02}\end{array}$ & $\begin{array}{l}9.9 \times 10^{-01} \\
3.2 \times 10^{-01}\end{array}$ & $\begin{array}{l}1.2 \times 10^{+44} \\
3.9 \times 10^{+43}\end{array}$ & $\begin{array}{r}7.9 \\
26.4\end{array}$ & $\begin{array}{l}1.1 \\
4.8 \\
\end{array}$ & $\begin{array}{l}3.8 \times 10^{-03} \\
2.9 \times 10^{-04} \\
\end{array}$ & $\begin{array}{l}3.9 \times 10^{-04} \\
2.8 \times 10^{-05} \\
\end{array}$ \\
\hline TAU 01 & 50 & $\begin{array}{l}\mathrm{R} \\
\mathrm{B}\end{array}$ & & & & $\begin{array}{l}2.1 \times 10^{-03} \\
2.6 \times 10^{-04}\end{array}$ & $\begin{array}{l}5.4 \times 10^{-03} \\
8.5 \times 10^{-04} \\
\end{array}$ & $\begin{array}{l}1.8 \times 10^{+41} \\
3.9 \times 10^{+40}\end{array}$ & $\begin{array}{r}26.4 \\
3.4 \\
\end{array}$ & $\begin{array}{r}12.6 \\
1.2 \\
\end{array}$ & $\begin{array}{l}1.9 \times 10^{-06} \\
3.2 \times 10^{-06}\end{array}$ & $\begin{array}{l}7.5 \times 10^{-07} \\
9.5 \times 10^{-07}\end{array}$ \\
\hline TAU 02 & 50 & $\begin{array}{l}\mathrm{R} \\
\mathrm{B}\end{array}$ & & & $\begin{array}{l}4.9 \\
6.6 \\
\end{array}$ & & $\begin{array}{l}5.6 \times 10^{-04} \\
6.2 \times 10^{-04} \\
\end{array}$ & $\begin{array}{l}1.2 \times 10^{+40} \\
1.9 \times 10^{+40} \\
\end{array}$ & & $\begin{array}{r}17.9 \\
8.5 \\
\end{array}$ & $\begin{array}{l}1.4 \times 10^{-07} \\
3.2 \times 10^{-07} \\
\end{array}$ & $\begin{array}{l}7.7 \times 10^{-08} \\
1.3 \times 10^{-07} \\
\end{array}$ \\
\hline TAU 03 & 50 & $\begin{array}{l}\mathrm{R} \\
\mathrm{B} \\
\end{array}$ & & & & & & & & $\begin{array}{r}8.5 \\
30.2 \\
\end{array}$ & $\begin{array}{l}6.5 \times 10^{-07} \\
1.6 \times 10^{-08} \\
\end{array}$ & $\begin{array}{l}3.2 \times 10^{-07} \\
5.0 \times 10^{-09}\end{array}$ \\
\hline TAU 04 & 50 & $\begin{array}{l}\mathrm{R} \\
\mathrm{B} \\
\end{array}$ & & $\begin{array}{r}18.0 \\
1.0 \\
\end{array}$ & $\begin{array}{r}11.7 \\
5.3 \\
\end{array}$ & $\begin{array}{l}9.5 \times 10^{-04} \\
4.7 \times 10^{-04} \\
\end{array}$ & $\begin{array}{l}2.9 \times 10^{-03} \\
6.5 \times 10^{-04} \\
\end{array}$ & $\begin{array}{l}1.3 \times 10^{+41} \\
1.2 \times 10^{+40} \\
\end{array}$ & & $\begin{array}{r}6.6 \\
24.8 \\
\end{array}$ & $\begin{array}{l}1.9 \times 10^{-06} \\
1.2 \times 10^{-07} \\
\end{array}$ & $\begin{array}{l}6.3 \times 10^{-07} \\
8.3 \times 10^{-08} \\
\end{array}$ \\
\hline TAU 06 & 30 & $\begin{array}{l}\mathrm{R} \\
\mathrm{B}\end{array}$ & $\begin{array}{l}9.0 \\
5.5\end{array}$ & $\begin{array}{r}14.0 \\
0.0 \\
\end{array}$ & $\begin{array}{l}6.8 \\
7.2 \\
\end{array}$ & $\begin{array}{l}5.4 \times 10^{-04} \\
5.3 \times 10^{-04}\end{array}$ & $\begin{array}{l}1.5 \times 10^{-03} \\
1.3 \times 10^{-03} \\
\end{array}$ & $\begin{array}{l}4.6 \times 10^{+40} \\
3.7 \times 10^{+40}\end{array}$ & $\begin{array}{l}26.4 \\
23.6 \\
\end{array}$ & $\begin{array}{l}18.4 \\
15.5 \\
\end{array}$ & $\begin{array}{l}2.2 \times 10^{-07} \\
2.3 \times 10^{-07}\end{array}$ & $\begin{array}{l}8.3 \times 10^{-08} \\
9.5 \times 10^{-08}\end{array}$ \\
\hline TAU 07 & $-^{c}$ & - & - & - & - & - & - & - & - & - & - & - \\
\hline TAU 08 & $--^{c}$ & - & - & - & - & - & - & - & - & - & - & - \\
\hline TAU 09 & $-^{c}$ & - & - & - & - & - & - & - & - & - & - & - \\
\hline W40 01 & $-^{c}$ & - & - & - & - & - & - & - & - & - & - & - \\
\hline W40 02 & 70 & $\begin{array}{l}\mathrm{R} \\
\mathrm{B} \\
\end{array}$ & $\begin{array}{r}11.5 \\
2.5 \\
\end{array}$ & $\begin{array}{r}19.0 \\
-6.5 \\
\end{array}$ & $\begin{array}{l}14.2 \\
11.3 \\
\end{array}$ & $\begin{array}{l}2.4 \times 10^{-03} \\
1.1 \times 10^{-02} \\
\end{array}$ & $\begin{array}{l}2.1 \times 10^{-02} \\
4.8 \times 10^{-02} \\
\end{array}$ & $\begin{array}{l}1.9 \times 10^{+42} \\
2.5 \times 10^{+42} \\
\end{array}$ & $\begin{array}{l}15.8 \\
26.4 \\
\end{array}$ & $\begin{array}{r}5.3 \\
11.0 \\
\end{array}$ & $\begin{array}{l}2.8 \times 10^{-05} \\
3.1 \times 10^{-05} \\
\end{array}$ & $\begin{array}{l}3.2 \times 10^{-06} \\
6.9 \times 10^{-06} \\
\end{array}$ \\
\hline W40 03 & $-^{c}$ & - & - & - & - & - & - & - & - & - & - & - \\
\hline W40 04 & $-^{c}$ & - & - & - & - & - & - & - & - & - & - & - \\
\hline W40 05 & $--^{c}$ & - & - & - & - & - & - & - & - & - & - & - \\
\hline W40 06 & $--^{c}$ & - & - & - & - & - & - & - & - & - & - & - \\
\hline W40 07 & 50 & $\begin{array}{l}\mathrm{R} \\
\mathrm{B}\end{array}$ & $\begin{array}{r}10.5 \\
3.0 \\
\end{array}$ & $\begin{array}{l}26.0 \\
-4.0 \\
\end{array}$ & $\begin{array}{l}18.6 \\
11.4 \\
\end{array}$ & $\begin{array}{l}5.4 \times 10^{-03} \\
1.2 \times 10^{-03}\end{array}$ & $\begin{array}{l}4.6 \times 10^{-02} \\
8.7 \times 10^{-03}\end{array}$ & $\begin{array}{l}5.1 \times 10^{+42} \\
6.9 \times 10^{+41}\end{array}$ & $\begin{array}{r}26.4 \\
4.2 \\
\end{array}$ & $\begin{array}{l}6.7 \\
1.7 \\
\end{array}$ & $\begin{array}{l}3.0 \times 10^{-05} \\
2.2 \times 10^{-05}\end{array}$ & $\begin{array}{l}3.5 \times 10^{-06} \\
3.0 \times 10^{-06}\end{array}$ \\
\hline
\end{tabular}

The results for all sources are presented in Table A.9, and Fig. A.3 shows the central $\mathrm{HCO}^{+}$and $\mathrm{C}^{18} \mathrm{O}$ spectra for all sources.

\section{A.7. Evolutionary classification}

Table A.10 presents a summary of the various indicators used to reach the final classification of the evolutionary state of sources in the WILL sample. Firstly, we consider whether or not an entrained molecular outflow is associated with the source in $\mathrm{CO}$ $J=3-2$, and whether or not there are broad line-wings in the HIFI water and CO $J=10-9$ spectra. Sources with all three signatures are likely to be the youngest protostars, with strong, energetic and likely warm outflows. Those without any detected outflow signatures are likely either pre-stellar or more evolved (i.e. Class II) sources.

Next, we follow the method of van Kempen et al. (2009) and Carney et al. (2016) using maps of the molecular emission in $\mathrm{HCO}^{+} J=4-3$ and $\mathrm{C}^{18} \mathrm{O} J=3-2$ to separate Class $0 / \mathrm{I} \mathrm{em}-$ bedded protostars from edge-on Class II disk sources. If both transitions are strong and spatially concentrated then the source is most likely a genuine embedded (i.e. Class 0/I) YSO. If not, the source is either too cold (i.e. pre-stellar) or does not have a significant envelope and so is a more evolved disk source. In the W40 sources, the extended emission in both lines is likely due to the PDR and may mask the presence of compact emission associated with the sources, so they are designated as confused. A few other sources also receive this designation, in line with Carney et al. (2016), if there are multiple sources in the field that cannot be disentangled at the resolution of the observations.

The presence of strong, compact sub-mm continuum emission is also indicative of a young (i.e. pre-stellar or embedded Class 0/I) source, allowing pre-stellar and Class II sources to be distinguished (e.g. André et al. 2000). Finally, the $T_{\text {bol }}$ classification (i.e. $T_{\text {bol }}<70 \mathrm{~K}$ corresponds to Class $0,70 \leq T_{\text {bol }}<650 \mathrm{~K}$ to Class I) can be used to separate Class 0 and I sources, though on its own this calculation is not always able to correctly separate other highly-reddened sources; for example edge-on Class II disks (Crapsi et al. 2008).

Overall, the WILL sample consists of 23 Class 0, 14 Class I, 8 Class II and 4 potentially pre-stellar sources. Most of the Class II sources are in Taurus, while the pre-stellar sources are all in Aquila/W40. Six sources (one Class 0, one Class II and four pre-stellar) have narrow yet bright ${ }^{12} \mathrm{CO} J=10-9$ emission (see Fig. 3) suggestive of a PDR along the line of sight to the source. The details of some of the more complex determinations are discussed in Appendix C. 
Table A.7. Mass-accretion rate, the luminosity of [O I] in the $63 \mu \mathrm{m}$ line, and mass-loss rate calculated from it.

\begin{tabular}{|c|c|c|c|c|c|c|c|}
\hline Name & $\begin{array}{c}\dot{M}_{\mathrm{acc}} \\
\left(M_{\odot} \mathrm{yr}^{-1}\right)\end{array}$ & $\begin{array}{c}L[\mathrm{O} \text { I } 63 \mu \mathrm{m}] \\
\left(L_{\odot}\right)\end{array}$ & $\begin{array}{c}\dot{M}_{\mathrm{w}} \\
\left(M_{\odot} \mathrm{yr}^{-1}\right)\end{array}$ & Name & $\begin{array}{c}\dot{M}_{\mathrm{acc}} \\
\left(M_{\odot} \mathrm{yr}^{-1}\right)\end{array}$ & $\begin{array}{c}L[\mathrm{O} \text { I } 63 \mu \mathrm{m}] \\
\left(L_{\odot}\right)\end{array}$ & $\begin{array}{c}\dot{M}_{\mathrm{w}} \\
\left(M_{\odot} \mathrm{yr}^{-1}\right)\end{array}$ \\
\hline AQU 01 & $1.7 \times 10^{-06}$ & $8.3 \times 10^{-04}$ & $8.3 \times 10^{-08}$ & AQU 02 & $5.9 \times 10^{-06}$ & $8.2 \times 10^{-04}$ & $8.2 \times 10^{-08}$ \\
\hline AQU 03 & $-^{a}$ & $-^{b}$ & - & AQU 04 & $8.4 \times 10^{-07}$ & $-^{b}$ & - \\
\hline AQU 05 & $1.6 \times 10^{-06}$ & $-^{b}$ & - & AQU 06 & $8.1 \times 10^{-07}$ & $-^{b}$ & - \\
\hline CHA 01 & ${ }^{a}$ & $3.7 \times 10^{-04}$ & $3.7 \times 10^{-08}$ & CHA 02 & $2.3 \times 10^{-07}$ & $8.7 \times 10^{-05}$ & $8.7 \times 10^{-09}$ \\
\hline CRA 01 & $1.6 \times 10^{-06}$ & $1.2 \times 10^{-03}$ & $1.2 \times 10^{-07}$ & ОРН 01 & $-^{a}$ & $2.9 \times 10^{-03 c}$ & - \\
\hline OPH 02 & $1.1 \times 10^{-06}$ & $5.2 \times 10^{-04}$ & $5.2 \times 10^{-08}$ & PER 01 & $2.9 \times 10^{-06}$ & $3.4 \times 10^{-03}$ & $3.4 \times 10^{-07}$ \\
\hline PER 02 & $6.0 \times 10^{-06}$ & $7.8 \times 10^{-03}$ & $7.8 \times 10^{-07}$ & PER 04 & $7.6 \times 10^{-07}$ & $5.3 \times 10^{-04}$ & $5.3 \times 10^{-08}$ \\
\hline PER 05 & $1.4 \times 10^{-06}$ & $2.8 \times 10^{-04}$ & $2.8 \times 10^{-08}$ & PER 06 & $9.2 \times 10^{-07}$ & $1.6 \times 10^{-03}$ & $1.6 \times 10^{-07}$ \\
\hline PER 07 & $4.6 \times 10^{-07}$ & $1.2 \times 10^{-03}$ & $1.2 \times 10^{-07}$ & PER 08 & $2.2 \times 10^{-06}$ & $1.1 \times 10^{-02}$ & $1.1 \times 10^{-06}$ \\
\hline PER 09 & $2.9 \times 10^{-06}$ & $-^{e}$ & - & PER 10 & $3.9 \times 10^{-06}$ & $-^{e}$ & - \\
\hline PER 12 & $6.9 \times 10^{-07}$ & $1.1 \times 10^{-04}$ & $1.1 \times 10^{-08}$ & PER 13 & $4.4 \times 10^{-07}$ & $-^{b}$ & - \\
\hline PER 14 & $2.4 \times 10^{-07}$ & $4.6 \times 10^{-04}$ & $4.6 \times 10^{-08}$ & PER 15 & $1.1 \times 10^{-06}$ & $2.1 \times 10^{-04}$ & $2.1 \times 10^{-08}$ \\
\hline PER 16 & $7.4 \times 10^{-07}$ & $2.6 \times 10^{-04}$ & $2.6 \times 10^{-08}$ & PER 17 & $2.3 \times 10^{-08}$ & $1.4 \times 10^{-04}$ & $1.4 \times 10^{-08}$ \\
\hline PER 18 & $3.4 \times 10^{-07}$ & $1.8 \times 10^{-03}$ & $1.8 \times 10^{-07}$ & PER 19 & $1.4 \times 10^{-07}$ & $1.8 \times 10^{-03}$ & $1.8 \times 10^{-07}$ \\
\hline PER 20 & $1.4 \times 10^{-06}$ & $1.9 \times 10^{-03}$ & $1.9 \times 10^{-07}$ & PER 21 & $1.2 \times 10^{-06}$ & $8.6 \times 10^{-04}$ & $8.6 \times 10^{-08}$ \\
\hline PER 22 & $1.6 \times 10^{-06}$ & $1.0 \times 10^{-03}$ & $1.0 \times 10^{-07}$ & SCO 01 & $-^{a}$ & $2.8 \times 10^{-04}$ & $2.8 \times 10^{-08}$ \\
\hline SERS 01 & $1.1 \times 10^{-05}$ & $2.8 \times 10^{-03}$ & $2.8 \times 10^{-07}$ & SERS 02 & $4.7 \times 10^{-05}$ & $-^{e}$ & - \\
\hline TAU 01 & $2.0 \times 10^{-07}$ & $7.6 \times 10^{-04}$ & $7.6 \times 10^{-08}$ & TAU 02 & $6.1 \times 10^{-08}$ & $1.1 \times 10^{-04}$ & $1.1 \times 10^{-08}$ \\
\hline TAU 03 & $-^{a}$ & $5.4 \times 10^{-05}$ & $5.4 \times 10^{-09}$ & TAU 04 & $1.8 \times 10^{-07}$ & $4.5 \times 10^{-04}$ & $4.5 \times 10^{-08}$ \\
\hline TAU 06 & $7.3 \times 10^{-08}$ & $9.6 \times 10^{-05}$ & $9.6 \times 10^{-09}$ & TAU 07 & $-^{a}$ & $3.5 \times 10^{-04}$ & $3.5 \times 10^{-08}$ \\
\hline TAU 08 & $-^{a}$ & $-{ }^{f}$ & - & TAU 09 & $-^{a}$ & $2.9 \times 10^{-04}$ & $2.9 \times 10^{-08}$ \\
\hline W40 01 & $-^{a}$ & $3.1 \times 10^{-04 c}$ & - & W4002 & $2.1 \times 10^{-05}$ & $5.4 \times 10^{-02}$ & $5.4 \times 10^{-06}$ \\
\hline W40 03 & $-^{a}$ & $1.5 \times 10^{-01 c}$ & - & W40 04 & ${ }^{a}$ & $1.1 \times 10^{-01 c}$ & - \\
\hline W40 05 & $-^{a}$ & $7.8 \times 10^{-02 c}$ & - & W4006 & $-^{a}$ & $-^{c, d}$ & - \\
\hline W40 07 & $2.3 \times 10^{-06}$ & $5.4 \times 10^{-04}$ & $5.4 \times 10^{-08}$ & L1448-MM & $5.8 \times 10^{-06}$ & $2.2 \times 10^{-03}$ & $2.2 \times 10^{-07}$ \\
\hline IRAS03245+3002 & $4.2 \times 10^{-06}$ & $6.4 \times 10^{-04}$ & $6.4 \times 10^{-08}$ & L1455-IRS3 & $4.7 \times 10^{-08}$ & $5.0 \times 10^{-04}$ & $5.0 \times 10^{-08}$ \\
\hline NGC 1333-IRAS2A & $2.3 \times 10^{-05}$ & $-^{b}$ & - & NGC 1333-IRAS3A & $5.4 \times 10^{-06}$ & $-^{f}$ & - \\
\hline NGC 1333-IRAS4A & $5.9 \times 10^{-06}$ & $1.5 \times 10^{-03 g}$ & - & NGC 1333-IRAS4B & $2.9 \times 10^{-06}$ & $1.3 \times 10^{-04}$ & $1.3 \times 10^{-08}$ \\
\hline IRAS03301+3111 & $5.3 \times 10^{-07}$ & $3.6 \times 10^{-04}$ & $3.6 \times 10^{-08}$ & $\mathrm{~B} 1 \mathrm{a}$ & $3.5 \times 10^{-07}$ & $1.9 \times 10^{-04}$ & $1.9 \times 10^{-08}$ \\
\hline $\mathrm{B} 1 \mathrm{c}$ & $3.3 \times 10^{-06}$ & $-{ }^{b}$ & - & L1489 & $4.9 \times 10^{-07}$ & $2.2 \times 10^{-04}$ & $2.2 \times 10^{-08}$ \\
\hline L1551-IRS5 & $2.9 \times 10^{-06}$ & $9.7 \times 10^{-03}$ & $9.7 \times 10^{-07}$ & TMR1 & $4.9 \times 10^{-07}$ & $1.1 \times 10^{-03}$ & $1.1 \times 10^{-07}$ \\
\hline TMC1A & $3.5 \times 10^{-07}$ & $3.3 \times 10^{-03}$ & $3.3 \times 10^{-07}$ & L1527 & $1.2 \times 10^{-06}$ & $1.8 \times 10^{-03}$ & $1.8 \times 10^{-07}$ \\
\hline TMC1 & $1.2 \times 10^{-07}$ & $1.5 \times 10^{-03}$ & $1.5 \times 10^{-07}$ & IRAM04191 & $7.1 \times 10^{-08}$ & $2.4 \times 10^{-04}$ & $2.4 \times 10^{-08}$ \\
\hline HH46 & $3.6 \times 10^{-06}$ & $5.1 \times 10^{-03}$ & $5.1 \times 10^{-07}$ & Ced110-IRS4 & $5.2 \times 10^{-07}$ & $1.3 \times 10^{-03}$ & $1.3 \times 10^{-07}$ \\
\hline BHR71 & $9.6 \times 10^{-06}$ & $3.4 \times 10^{-03}$ & $3.4 \times 10^{-07}$ & IRAS12496 & $4.6 \times 10^{-06}$ & $5.9 \times 10^{-03}$ & $5.9 \times 10^{-07}$ \\
\hline IRAS15398 & $1.0 \times 10^{-06}$ & $3.0 \times 10^{-03}$ & $3.0 \times 10^{-07}$ & GSS30 & $1.8 \times 10^{-06}$ & $5.1 \times 10^{-03}$ & $5.1 \times 10^{-07}$ \\
\hline VLA1623-243 & $1.7 \times 10^{-06}$ & $1.3 \times 10^{-03}$ & $1.3 \times 10^{-07}$ & WL12 & $2.1 \times 10^{-07}$ & $7.9 \times 10^{-04}$ & $7.9 \times 10^{-08}$ \\
\hline Elias29 & $1.8 \times 10^{-06}$ & $4.0 \times 10^{-03}$ & $4.0 \times 10^{-07}$ & IRS44 & $6.6 \times 10^{-07}$ & $4.8 \times 10^{-04}$ & $4.8 \times 10^{-08}$ \\
\hline IRS46 & $6.5 \times 10^{-08}$ & $1.5 \times 10^{-04}$ & $1.5 \times 10^{-08}$ & IRS63 & $1.3 \times 10^{-07}$ & $3.3 \times 10^{-04}$ & $3.3 \times 10^{-08}$ \\
\hline RNO91 & $3.4 \times 10^{-07}$ & $1.3 \times 10^{-03}$ & $1.3 \times 10^{-07}$ & L483 & $6.6 \times 10^{-06}$ & $1.1 \times 10^{-03}$ & $1.1 \times 10^{-07}$ \\
\hline Ser-SMM1 & $7.1 \times 10^{-05}$ & $4.0 \times 10^{-03}$ & $4.0 \times 10^{-07}$ & Ser-SMM4 & $4.4 \times 10^{-06}$ & $1.8 \times 10^{-02}$ & $1.8 \times 10^{-06}$ \\
\hline Ser-SMM3 & $1.2 \times 10^{-05}$ & $1.1 \times 10^{-02}$ & $1.1 \times 10^{-06}$ & RCrA-IRS5A & $9.2 \times 10^{-07}$ & $1.2 \times 10^{-03}$ & $1.2 \times 10^{-07}$ \\
\hline RCrA I7A & $1.2 \times 10^{-05}$ & $8.9 \times 10^{-03}$ & $8.9 \times 10^{-07}$ & RCrA I7B & $6.0 \times 10^{-06}$ & $2.4 \times 10^{-03}$ & $2.4 \times 10^{-07}$ \\
\hline HH100 & $2.3 \times 10^{-06}$ & $--^{f}$ & - & L723 & $2.3 \times 10^{-06}$ & $1.3 \times 10^{-04}$ & $1.3 \times 10^{-08}$ \\
\hline B335 & $2.1 \times 10^{-06}$ & $2.5 \times 10^{-04}$ & $2.5 \times 10^{-08}$ & L1157 & $3.0 \times 10^{-06}$ & $3.3 \times 10^{-04}$ & $3.3 \times 10^{-08}$ \\
\hline
\end{tabular}

Notes. ${ }^{(a)}$ Not Class 0/I. ${ }^{(b)}$ Non-detection. ${ }^{(c)}$ Dominated by PDR. ${ }^{(d)}$ Line in absorption. ${ }^{(e)}$ Dominated by neighbouring source. ${ }^{(f)}$ Source not observed with PACS. ${ }^{(g)}$ Only one off position was used due to possible contamination but this flux may still be underestimated. 
J. C. Mottram et al.: Outflows, infall and evolution of a sample of embedded low-mass protostars
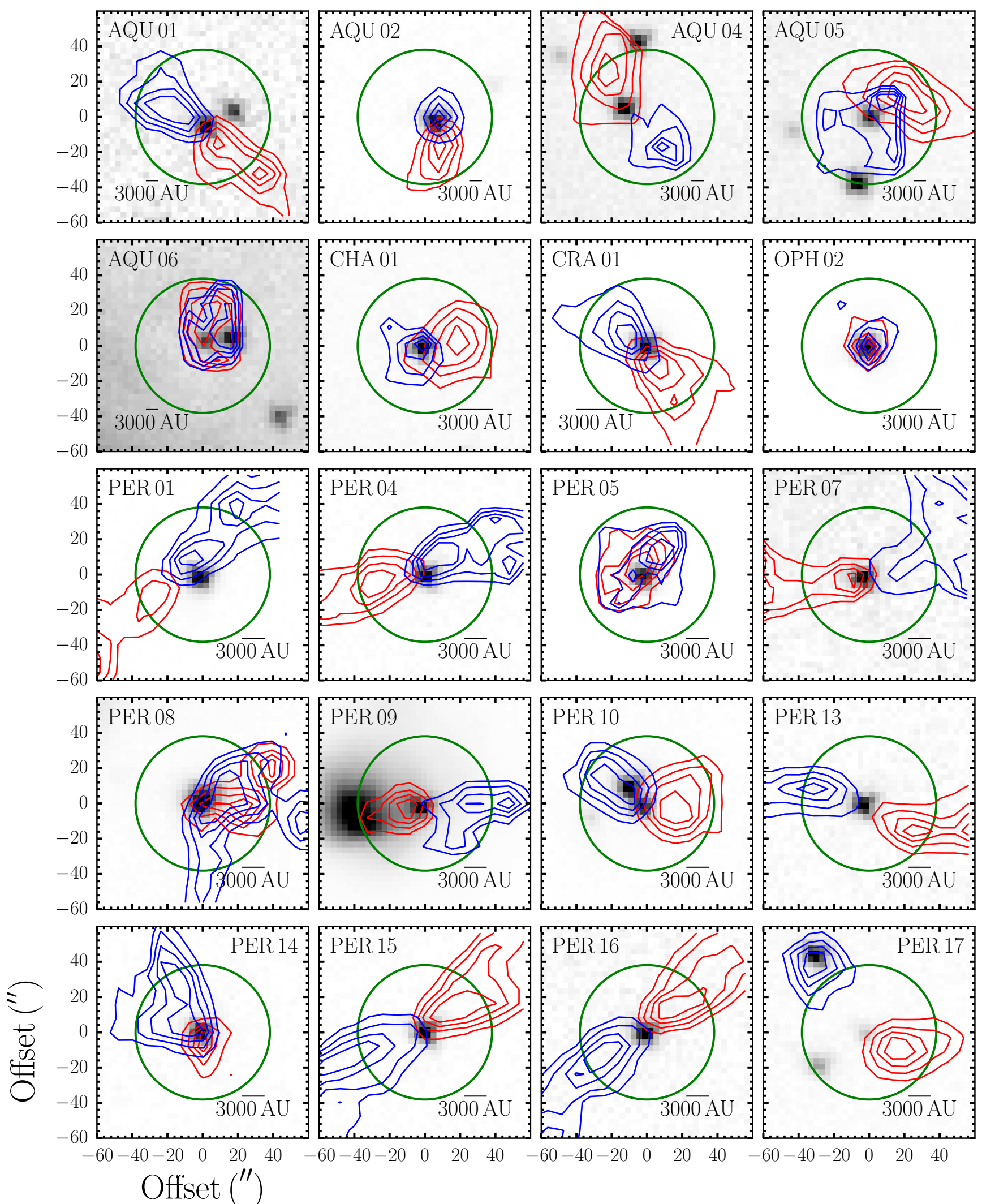

Fig. A.2. Outflow maps. The grey-scale images show the $70 \mu \mathrm{m}$ continuum emission from Herschel, while the red and blue contours show the outflow lobes detected in ${ }^{12} \mathrm{CO} J=3-2$. The levels for the contours are at 20, 40, 60 and $80 \%$ of the maximum velocity-integrated emission. The green circle indicates the HIFI beam for the $\mathrm{H}_{2} \mathrm{O} 1_{10}-1_{01}$ transition. All maps show a region of $2^{\prime} \times 2^{\prime}$ centred on the source position. The black scale-bar in the lower panel of each figure indicates $3000 \mathrm{AU}$ at the distance of the source. 


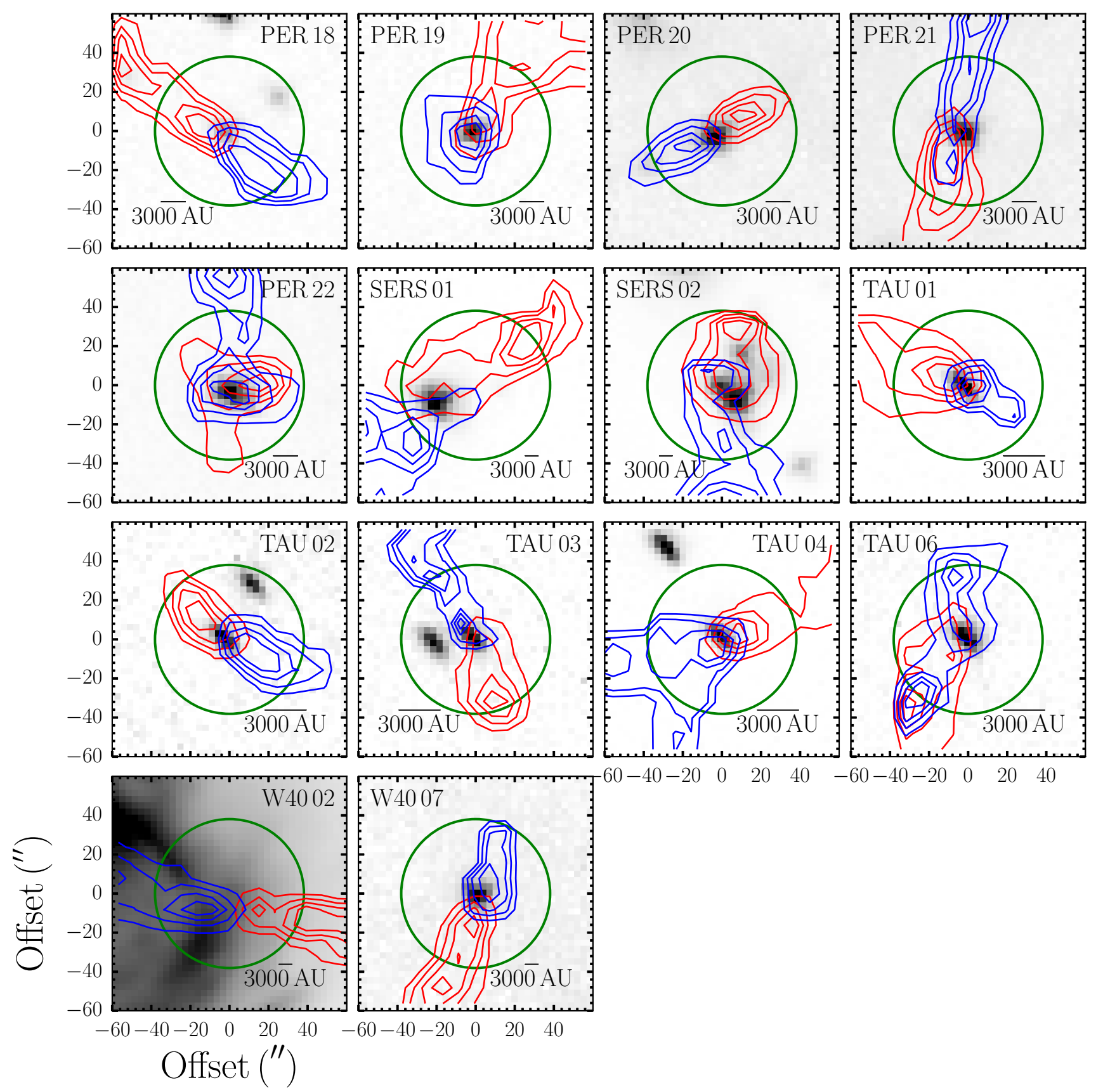

Fig. A.2. continued. 
J. C. Mottram et al.: Outflows, infall and evolution of a sample of embedded low-mass protostars

Table A.8. Species and transitions targeted during the ground-based spectral follow-up.

\begin{tabular}{|c|c|c|c|c|c|c|c|c|c|c|}
\hline Species & Transition & $\begin{array}{l}\text { Rest frequency }{ }^{a} \\
(\mathrm{GHz})\end{array}$ & $\begin{array}{l}E_{\mathrm{u}} / k_{\mathrm{b}} \\
(\mathrm{K})\end{array}$ & $\begin{array}{c}A_{\mathrm{ul}} \\
\left(\mathrm{s}^{-1}\right)\end{array}$ & $\begin{array}{c}n_{\mathrm{cr}} \\
\left(\mathrm{cm}^{-3}\right)\end{array}$ & $\mathrm{JCMT} \eta_{\mathrm{mb}}{ }^{b}$ & $\operatorname{APEX} \eta_{\mathrm{mb}}{ }^{c}$ & $\begin{array}{c}\mathrm{JCMT} \theta_{\mathrm{mb}} \\
\left({ }^{\prime \prime}\right)\end{array}$ & $\begin{array}{l}\text { APEX } \theta_{\mathrm{mb}} \\
\left({ }^{\prime \prime}\right)\end{array}$ & $\begin{array}{c}\text { Median } \sigma_{\text {rms }}{ }^{d} \\
(\mathrm{~K})\end{array}$ \\
\hline $\mathrm{CO}$ & $2-1$ & 230.53800 & 16.60 & $6.91 \times 10^{-7}$ & $6 \times 10^{3}$ & - & 0.75 & - & 27.3 & 0.41 \\
\hline${ }^{13} \mathrm{CO}$ & $2-1$ & 220.39868 & 15.87 & $6.04 \times 10^{-7}$ & $5 \times 10^{3}$ & - & 0.75 & - & 28.5 & 0.08 \\
\hline $\mathrm{C}^{18} \mathrm{O}$ & $2-1$ & 219.56035 & 15.81 & $6.01 \times 10^{-7}$ & $5 \times 10^{3}$ & - & 0.75 & - & 28.6 & 0.08 \\
\hline $\mathrm{C}^{17} \mathrm{O}$ & $2-1$ & 224.71439 & 16.18 & $6.43 \times 10^{-7}$ & $6 \times 10^{3}$ & - & 0.75 & - & 28.0 & 0.08 \\
\hline $\mathrm{CO}$ & $3-2$ & 345.79599 & 33.19 & $2.50 \times 10^{-6}$ & $2 \times 10^{4}$ & 0.63 & 0.73 & 14.5 & 18.2 & 0.09 \\
\hline${ }^{13} \mathrm{CO}$ & $3-2$ & 330.58797 & 31.73 & $2.18 \times 10^{-6}$ & $1 \times 10^{4}$ & 0.63 & 0.73 & 15.2 & 19.0 & 0.22 \\
\hline $\mathrm{C}^{18} \mathrm{O}$ & $3-2$ & 329.33055 & 31.61 & $2.17 \times 10^{-6}$ & $1 \times 10^{4}$ & 0.63 & 0.73 & 15.3 & 19.1 & 0.30 \\
\hline $\mathrm{C}^{17} \mathrm{O}$ & $3-2$ & 337.06113 & 32.35 & $2.32 \times 10^{-6}$ & $1 \times 10^{4}$ & - & 0.73 & - & 18.7 & 0.09 \\
\hline $\mathrm{CO}$ & $4-3$ & 461.04077 & 55.32 & $6.13 \times 10^{-6}$ & $3 \times 10^{4}$ & - & 0.60 & - & 13.6 & 0.12 \\
\hline $\mathrm{HCO}^{+}$ & $4-3$ & 356.73429 & 42.80 & $3.63 \times 10^{-3}$ & $2 \times 10^{7}$ & 0.63 & 0.73 & 14.1 & 17.6 & 0.28 \\
\hline $\mathrm{H}^{13} \mathrm{CO}^{+}$ & $4-3$ & 346.99834 & 41.63 & $3.29 \times 10^{-3}$ & $2 \times 10^{7}$ & 0.63 & 0.73 & 14.5 & 18.2 & 0.13 \\
\hline
\end{tabular}

Notes. ${ }^{(a)}$ Taken from the JPL database (Pickett et al. 2010); ${ }^{(b)}$ historical value from long-term monitoring of standards used for consistency with

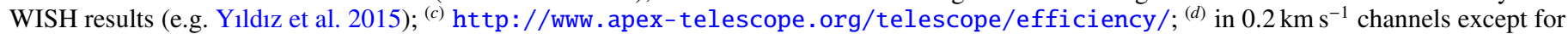
CO $J=3-2$, which is in $0.5 \mathrm{~km} \mathrm{~s}^{-1}$ channels. 


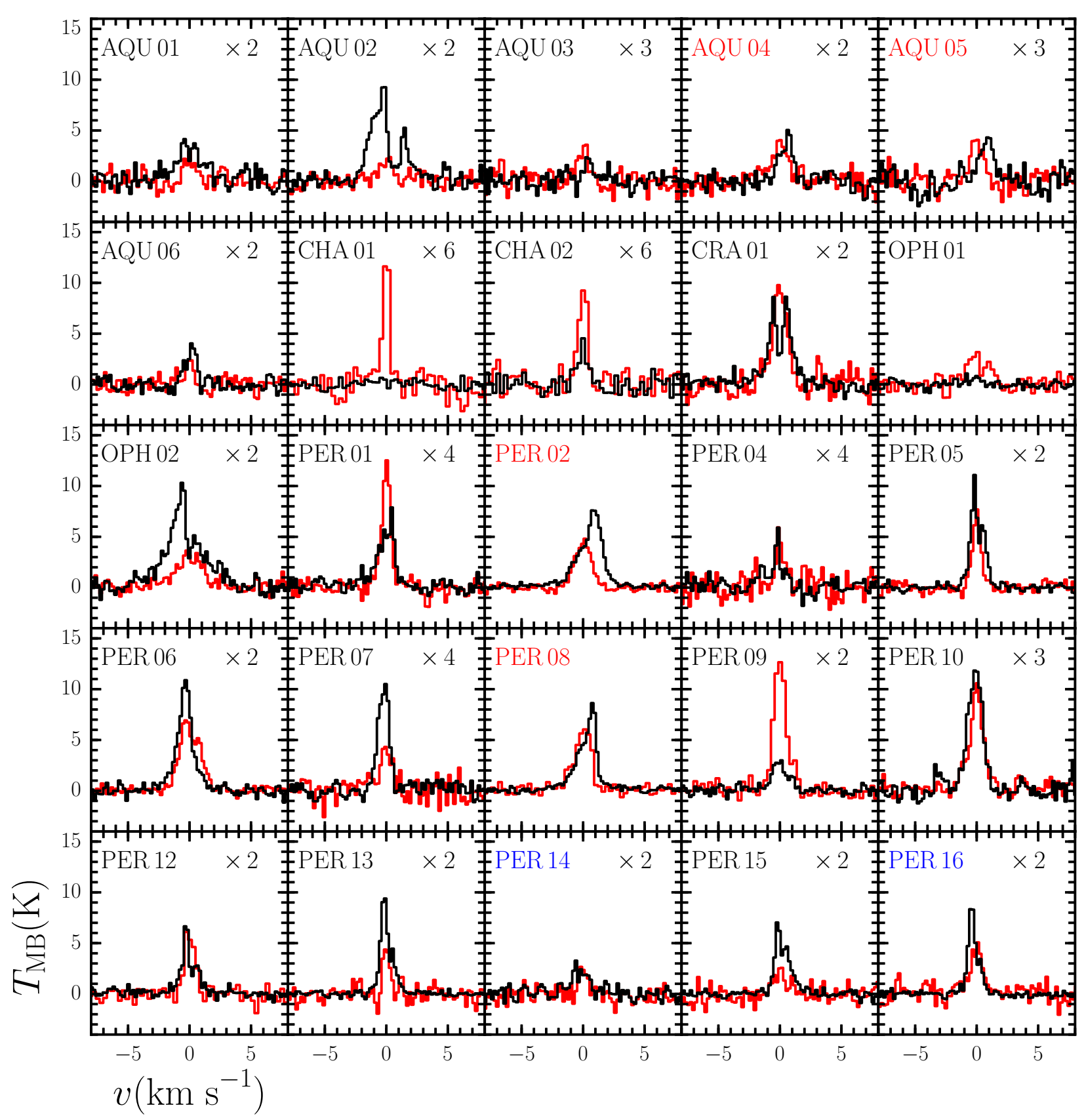

Fig. A.3. Overview of the central $\mathrm{HCO}^{+} J=4-3$ and $\mathrm{C}^{18} \mathrm{O} J=3-2$ spectra shown in black and red respectively. All have been recentred so that the source velocity is at zero. Sources that are considered blue-skewed (i.e. $\delta v<-0.25$ ) have their names in blue, while those considered red-skewed (i.e. $\delta v>0.25$ ) have their names in red. The number in the upper-right corner of each panel indicates what factor the spectra have been multiplied by. 
J. C. Mottram et al.: Outflows, infall and evolution of a sample of embedded low-mass protostars

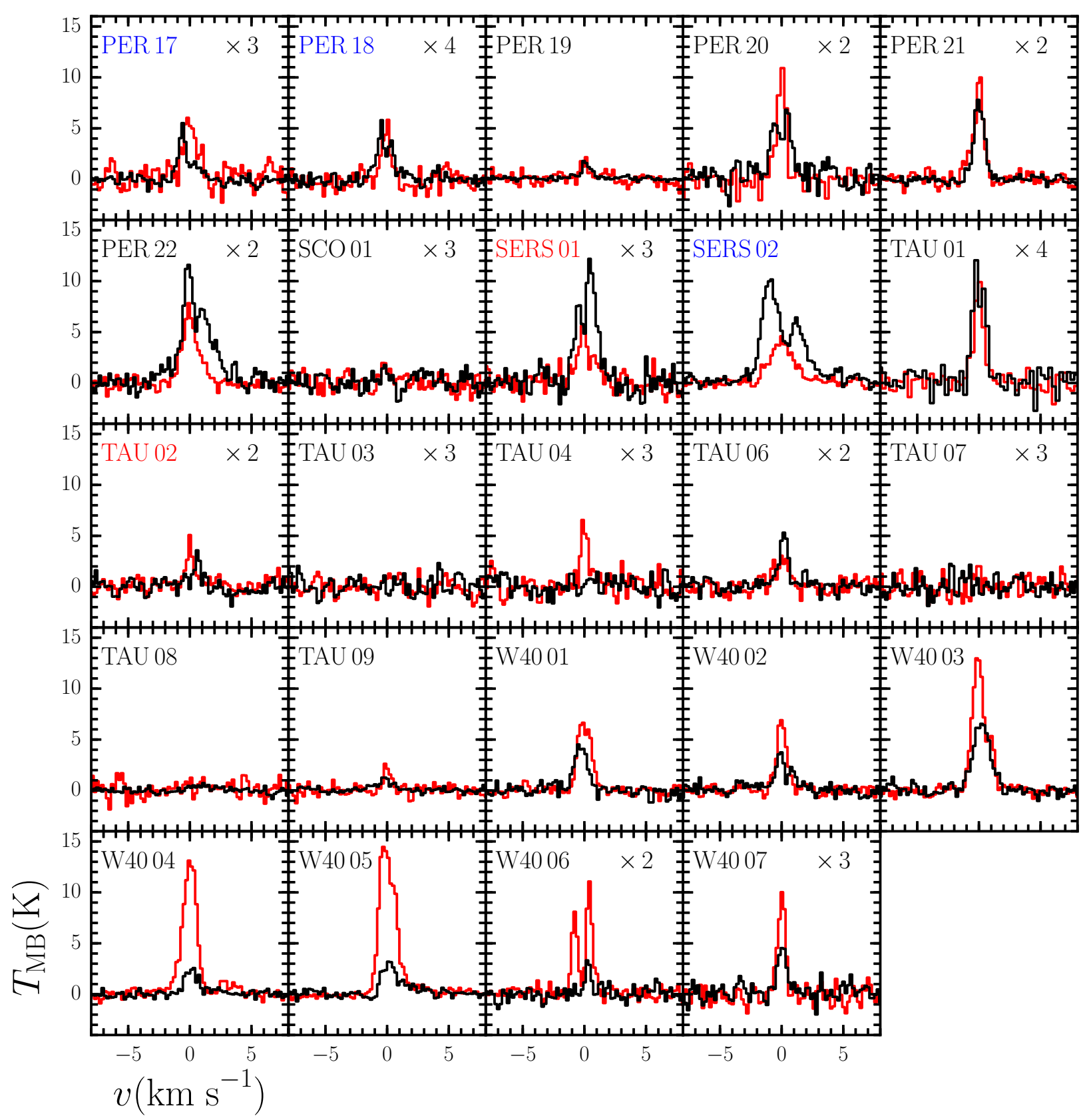

Fig. A.3. continued. 
Table A.9. Ground-based spectral results.

\begin{tabular}{|c|c|c|c|c|c|c|c|c|c|c|}
\hline Name & Molecule & Transition & $\begin{array}{c}\sigma_{\mathrm{rms}} \\
(\mathrm{K})\end{array}$ & $\begin{array}{c}v_{\max } \\
\left(\mathrm{km} \mathrm{s}^{-1}\right)\end{array}$ & $\begin{array}{l}T_{\max } \\
(\mathrm{K})\end{array}$ & $\begin{array}{c}v_{\text {peak }}{ }^{a} \\
\left(\mathrm{~km} \mathrm{~s}^{-1}\right)\end{array}$ & $\begin{array}{c}T_{\text {peak }^{a}} \\
\text { (K) }\end{array}$ & $\begin{array}{c}F W H M^{a} \\
\left(\mathrm{~km} \mathrm{~s}^{-1}\right)\end{array}$ & $\begin{array}{l}\int T_{\mathrm{MB}} \mathrm{d} v^{b} \\
\left(\mathrm{~K} \mathrm{~km} \mathrm{~s}^{-1}\right)\end{array}$ & $\begin{array}{c}\delta v^{c} \\
\left(\mathrm{~km} \mathrm{~s}^{-1}\right)\end{array}$ \\
\hline \multirow[t]{4}{*}{ AQU 01} & ${ }^{13} \mathrm{CO}$ & $3-2$ & 0.25 & 7.2 & 2.33 & $7.3 \pm 0.1$ & $2.1 \pm 0.2$ & $1.9 \pm 0.2$ & $4.23 \pm 0.57$ & $-0.07 \pm 0.03$ \\
\hline & $\mathrm{C}^{18} \mathrm{O}$ & $3-2$ & 0.32 & 7.4 & 1.48 & $7.4 \pm 0.2$ & $1.2 \pm 0.4$ & $1.1 \pm 0.4$ & $1.34 \pm 0.74$ & $0.00 \pm 0.01$ \\
\hline & $\mathrm{HCO}^{+}$ & $4-3$ & 0.39 & 7.0 & 2.08 & $7.4 \pm 0.2$ & $1.6 \pm 0.2$ & $2.6 \pm 0.4$ & $4.56 \pm 0.89$ & $-0.04 \pm 0.01$ \\
\hline & $\mathrm{H}^{13} \mathrm{CO}^{+}$ & $4-3$ & 0.18 & - & $<0.55$ & - & - & - & - & - \\
\hline \multirow{3}{*}{ AQU 02} & ${ }^{13} \mathrm{CO}$ & $3-2$ & 0.21 & 7.6 & 4.47 & $7.5 \pm 0.1$ & $4.2 \pm 0.2$ & $1.8 \pm 0.1$ & $7.95 \pm 0.48$ & $-0.01 \pm 0.01$ \\
\hline & $\mathrm{C}^{18} \mathrm{O}$ & $3-2$ & 0.26 & 7.8 & 1.19 & $7.5 \pm 0.2$ & $1.0 \pm 0.3$ & $1.2 \pm 0.4$ & $1.38 \pm 0.60$ & $0.00 \pm 0.01$ \\
\hline & $\mathrm{HCO}^{+d}$ & $4-3$ & 0.30 & 7.4 & 4.63 & $7.6 \pm 0.1$ & $4.7 \pm 0.3$ & $2.7 \pm 0.1$ & $13.28 \pm 0.94$ & $-0.09 \pm 0.03^{e}$ \\
\hline \multirow{4}{*}{ AQU 03} & ${ }^{13} \mathrm{CO}$ & $3-2$ & 0.21 & 7.4 & 2.33 & $7.1 \pm 0.1$ & $2.2 \pm 0.2$ & $1.4 \pm 0.2$ & $3.27 \pm 0.49$ & $0.02 \pm 0.01$ \\
\hline & $\mathrm{C}^{18} \mathrm{O}$ & $3-2$ & 0.28 & 7.4 & 1.20 & $7.1 \pm 0.2$ & $1.1 \pm 0.4$ & $1.1 \pm 0.4$ & $1.22 \pm 0.65$ & $0.00 \pm 0.01$ \\
\hline & $\mathrm{HCO}^{+}$ & $4-3$ & 0.28 & - & $<0.85$ & - & - & - & - & - \\
\hline & $\mathrm{H}^{13} \mathrm{CO}^{+}$ & $4-3$ & 0.25 & - & $<0.75$ & - & - & - & - & - \\
\hline \multirow{4}{*}{ AQU 04} & ${ }^{13} \mathrm{CO}$ & $3-2$ & 0.22 & 7.4 & 3.90 & $7.5 \pm 0.1$ & $3.2 \pm 0.1$ & $2.8 \pm 0.1$ & $9.29 \pm 0.55$ & $-0.01 \pm 0.01$ \\
\hline & $\mathrm{C}^{18} \mathrm{O}$ & $3-2$ & 0.36 & 7.6 & 2.03 & $7.6 \pm 0.1$ & $1.8 \pm 0.3$ & $1.6 \pm 0.3$ & $3.07 \pm 0.83$ & $0.00 \pm 0.01$ \\
\hline & $\mathrm{HCO}^{+}$ & $4-3$ & 0.33 & 8.2 & 2.52 & $8.1 \pm 0.1$ & $2.1 \pm 0.4$ & $1.3 \pm 0.3$ & $2.81 \pm 0.78$ & $0.33 \pm 0.07$ \\
\hline & $\mathrm{H}^{13} \mathrm{CO}^{+}$ & $4-3$ & 0.19 & - & $<0.56$ & - & - & - & - & - \\
\hline \multirow{4}{*}{ AQU 05} & ${ }^{13} \mathrm{CO}$ & $3-2$ & 0.27 & 7.0 & 2.79 & $7.4 \pm 0.1$ & $1.9 \pm 0.1$ & $2.9 \pm 0.2$ & $5.91 \pm 0.66$ & $0.10 \pm 0.03$ \\
\hline & $\mathrm{C}^{18} \mathrm{O}$ & $3-2$ & 0.30 & 7.4 & 1.36 & $7.3 \pm 0.2$ & $1.4 \pm 0.4$ & $1.2 \pm 0.4$ & $1.72 \pm 0.69$ & $0.00 \pm 0.01$ \\
\hline & $\mathrm{HCO}^{+}$ & $4-3$ & 0.31 & 8.2 & 1.44 & $8.2 \pm 0.2$ & $1.4 \pm 0.3$ & $1.3 \pm 0.4$ & $1.98 \pm 0.71$ & $0.70 \pm 0.21$ \\
\hline & $\mathrm{H}^{13} \mathrm{CO}^{+}$ & $4-3$ & 0.14 & - & $<0.43$ & - & - & - & - & - \\
\hline \multirow[t]{4}{*}{ AQU 06} & ${ }^{13} \mathrm{CO}$ & $3-2$ & 0.25 & 8.4 & 2.52 & $8.3 \pm 0.1$ & $2.0 \pm 0.2$ & $1.9 \pm 0.2$ & $4.11 \pm 0.57$ & $0.11 \pm 0.07$ \\
\hline & $\mathrm{C}^{18} \mathrm{O}$ & $3-2$ & 0.33 & 8.4 & 1.19 & $8.3 \pm 0.2$ & $1.3 \pm 0.7$ & $0.6 \pm 0.4$ & $0.90 \pm 0.76$ & $0.00 \pm 0.01$ \\
\hline & $\mathrm{HCO}^{+}$ & $4-3$ & 0.29 & 8.4 & 2.02 & $8.4 \pm 0.1$ & $1.8 \pm 0.4$ & $1.0 \pm 0.3$ & $1.96 \pm 0.67$ & $0.22 \pm 0.14$ \\
\hline & $\mathrm{H}^{13} \mathrm{CO}^{+}$ & $4-3$ & 0.16 & - & $<0.47$ & - & - & - & - & - \\
\hline \multirow[t]{8}{*}{ CHA 01} & ${ }^{13} \mathrm{CO}$ & $2-1$ & 0.08 & 5.2 & 6.63 & $4.9 \pm 0.1$ & $5.9 \pm 0.2$ & $1.5 \pm 0.1$ & $9.62 \pm 0.43$ & $-0.01 \pm 0.01$ \\
\hline & $\mathrm{C}^{18} \mathrm{O}$ & $2-1$ & 0.08 & 4.7 & 4.44 & $4.7 \pm 0.1$ & $4.8 \pm 0.3$ & $0.7 \pm 0.1$ & $3.63 \pm 0.39$ & $0.00 \pm 0.01$ \\
\hline & $\mathrm{C}^{17} \mathrm{O}$ & $2-1$ & 0.08 & 5.2 & 1.47 & $5.2 \pm 0.1$ & $1.4 \pm 0.3$ & $0.7 \pm 0.2$ & $1.09 \pm 0.37$ & $0.00 \pm 0.01$ \\
\hline & ${ }^{13} \mathrm{CO}$ & $3-2$ & 0.12 & 5.1 & 4.04 & $4.8 \pm 0.1$ & $3.7 \pm 0.2$ & $1.6 \pm 0.1$ & $6.43 \pm 0.55$ & $-0.13 \pm 0.05$ \\
\hline & $\mathrm{C}^{18} \mathrm{O}$ & $3-2$ & 0.16 & 4.8 & 1.94 & $4.9 \pm 0.1$ & $2.2 \pm 0.7$ & $0.6 \pm 0.2$ & $1.49 \pm 0.77$ & $0.00 \pm 0.01$ \\
\hline & $\mathrm{C}^{17} \mathrm{O}$ & $3-2$ & 0.08 & 5.1 & 0.45 & $4.8 \pm 0.2$ & $0.4 \pm 0.2$ & $0.9 \pm 0.6$ & $0.43 \pm 0.35$ & $0.00 \pm 0.01$ \\
\hline & $\mathrm{HCO}^{+}$ & $4-3$ & 0.07 & - & $<0.20$ & - & - & - & - & - \\
\hline & $\mathrm{H}^{13} \mathrm{CO}^{+}$ & $4-3$ & 0.06 & - & $<0.18$ & - & - & - & - & - \\
\hline \multirow[t]{8}{*}{ CHA 02} & ${ }^{13} \mathrm{CO}$ & $2-1$ & 0.09 & 3.5 & 4.66 & $3.2 \pm 0.1$ & $4.7 \pm 0.2$ & $1.3 \pm 0.1$ & $6.71 \pm 0.47$ & $0.18 \pm 0.07$ \\
\hline & $\mathrm{C}^{18} \mathrm{O}$ & $2-1$ & 0.08 & 3.1 & 3.60 & $3.0 \pm 0.1$ & $3.7 \pm 0.3$ & $0.8 \pm 0.1$ & $2.98 \pm 0.43$ & $0.00 \pm 0.01$ \\
\hline & $\mathrm{C}^{17} \mathrm{O}$ & $2-1$ & 0.08 & 3.5 & 1.36 & $3.5 \pm 0.1$ & $1.2 \pm 0.3$ & $0.8 \pm 0.2$ & $1.06 \pm 0.37$ & $0.00 \pm 0.01$ \\
\hline & ${ }^{13} \mathrm{CO}$ & $3-2$ & 0.13 & 3.6 & 2.24 & $3.0 \pm 0.1$ & $2.0 \pm 0.2$ & $1.8 \pm 0.2$ & $3.84 \pm 0.59$ & $-0.01 \pm 0.01$ \\
\hline & $\mathrm{C}^{18} \mathrm{O}$ & $3-2$ & 0.17 & 3.0 & 1.54 & $3.0 \pm 0.1$ & $1.6 \pm 0.5$ & $0.9 \pm 0.3$ & $1.47 \pm 0.75$ & $0.00 \pm 0.01$ \\
\hline & $\mathrm{C}^{17} \mathrm{O}$ & $3-2$ & 0.10 & 3.3 & 0.40 & $3.2 \pm 0.3$ & $0.4 \pm 0.3$ & $0.8 \pm 0.8$ & $0.31 \pm 0.43$ & $0.00 \pm 0.01$ \\
\hline & $\mathrm{HCO}^{+}$ & $4-3$ & 0.11 & 3.0 & 0.76 & $3.0 \pm 0.2$ & $0.6 \pm 0.3$ & $1.0 \pm 0.6$ & $0.65 \pm 0.49$ & $-0.04 \pm 0.02$ \\
\hline & $\mathrm{H}^{13} \mathrm{CO}^{+}$ & $4-3$ & 0.10 & - & $<0.29$ & - & - & - & - & - \\
\hline \multirow[t]{3}{*}{ CRA 01} & ${ }^{13} \mathrm{CO}$ & $3-2$ & 0.43 & 5.1 & 8.82 & $5.1 \pm 0.1$ & $7.2 \pm 0.5$ & $1.2 \pm 0.1$ & $9.22 \pm 1.05$ & $-0.41 \pm 0.05$ \\
\hline & $\mathrm{C}^{18} \mathrm{O}$ & $3-2$ & 0.51 & 5.5 & 4.89 & $5.6 \pm 0.1$ & $4.7 \pm 0.5$ & $1.4 \pm 0.2$ & $6.82 \pm 1.18$ & $0.00 \pm 0.01$ \\
\hline & $\mathrm{HCO}^{+}$ & $4-3$ & 0.31 & 6.1 & 4.32 & $5.7 \pm 0.1$ & $3.2 \pm 0.2$ & $2.3 \pm 0.2$ & $7.69 \pm 0.76$ & $0.04 \pm 0.01$ \\
\hline \multirow[t]{4}{*}{ OPH 01} & ${ }^{13} \mathrm{CO}$ & $3-2$ & 0.50 & 3.0 & 6.79 & $3.7 \pm 0.1$ & $6.9 \pm 0.4$ & $2.4 \pm 0.2$ & $17.87 \pm 1.49$ & $-0.07 \pm 0.02$ \\
\hline & $\mathrm{C}^{18} \mathrm{O}$ & $3-2$ & 0.61 & 3.9 & 3.18 & $3.8 \pm 0.2$ & $2.6 \pm 0.5$ & $2.1 \pm 0.5$ & $5.86 \pm 1.73$ & $0.00 \pm 0.01$ \\
\hline & $\mathrm{HCO}^{+}$ & $4-3$ & 0.34 & - & $<1.01$ & - & - & - & - & - \\
\hline & $\mathrm{H}^{13} \mathrm{CO}^{+}$ & $4-3$ & 0.15 & - & $<0.46$ & - & - & - & - & - \\
\hline \multirow[t]{4}{*}{ OPH 02} & ${ }^{13} \mathrm{CO}$ & $3-2$ & 0.22 & 4.6 & 7.84 & $4.4 \pm 0.1$ & $6.2 \pm 0.2$ & $2.5 \pm 0.1$ & $16.68 \pm 0.62$ & $0.08 \pm 0.01$ \\
\hline & $\mathrm{C}^{18} \mathrm{O}$ & $3-2$ & 0.27 & 4.0 & 1.82 & $4.2 \pm 0.1$ & $1.5 \pm 0.1$ & $2.6 \pm 0.3$ & $4.25 \pm 0.61$ & $0.00 \pm 0.01$ \\
\hline & $\mathrm{HCO}^{+}$ & $4-3$ & 0.32 & 3.6 & 5.16 & $3.6 \pm 0.1$ & $3.4 \pm 0.1$ & $3.4 \pm 0.2$ & $12.22 \pm 0.80$ & $-0.22 \pm 0.03$ \\
\hline & $\mathrm{H}^{13} \mathrm{CO}^{+}$ & $4-3$ & 0.13 & - & $<0.40$ & - & - & - & - & - \\
\hline \multirow[t]{3}{*}{ PER 01} & ${ }^{13} \mathrm{CO}$ & $3-2$ & 0.16 & 3.9 & 5.03 & $4.1 \pm 0.1$ & $4.9 \pm 0.2$ & $1.6 \pm 0.1$ & $8.24 \pm 0.41$ & $-0.00 \pm 0.01$ \\
\hline & $\mathrm{C}^{18} \mathrm{O}$ & $3-2$ & 0.15 & 4.1 & 3.13 & $4.1 \pm 0.1$ & $3.0 \pm 0.3$ & $0.8 \pm 0.1$ & $2.62 \pm 0.36$ & $0.00 \pm 0.01$ \\
\hline & $\mathrm{HCO}^{+}$ & $4-3$ & 0.15 & 4.5 & 1.97 & $4.1 \pm 0.1$ & $1.5 \pm 0.1$ & $1.6 \pm 0.2$ & $2.52 \pm 0.34$ & $0.06 \pm 0.01$ \\
\hline PER 02 & ${ }^{13} \mathrm{CO}$ & $3-2$ & 0.20 & 4.5 & 7.89 & $4.6 \pm 0.1$ & $7.9 \pm 0.1$ & $2.2 \pm 0.1$ & $18.84 \pm 0.48$ & $0.06 \pm 0.01$ \\
\hline & $\mathrm{C}^{18} \mathrm{O}$ & $3-2$ & 0.31 & 4.7 & 4.82 & $4.5 \pm 0.1$ & $4.5 \pm 0.3$ & $1.6 \pm 0.1$ & $7.60 \pm 0.70$ & $0.00 \pm 0.01$ \\
\hline & $\mathrm{HCO}^{+}$ & $4-3$ & 0.16 & 5.3 & 7.60 & $5.3 \pm 0.1$ & $6.7 \pm 0.1$ & $2.1 \pm 0.1$ & $15.10 \pm 0.48$ & $0.46 \pm 0.03$ \\
\hline & $\mathrm{H}^{13} \mathrm{CO}^{+}$ & $4-3$ & 0.16 & - & $<0.47$ & - & - & - & - & - \\
\hline PER 04 & $\mathrm{C}^{18} \mathrm{O}$ & $3-2$ & 0.26 & 5.1 & 1.48 & $5.2 \pm 0.2$ & $1.2 \pm 0.5$ & $0.8 \pm 0.4$ & $1.04 \pm 0.61$ & $0.00 \pm 0.01$ \\
\hline & $\mathrm{HCO}^{+}$ & $4-3$ & 0.16 & 5.1 & 1.48 & $4.9 \pm 0.1$ & $0.8 \pm 0.1$ & $1.6 \pm 0.3$ & $1.29 \pm 0.38$ & $-0.17 \pm 0.08^{e}$ \\
\hline & $\mathrm{H}^{13} \mathrm{CO}^{+}$ & $4-3$ & 0.12 & - & $<0.36$ & - & - & - & - & - \\
\hline PER 05 & ${ }^{13} \mathrm{CO}$ & $3-2$ & 0.24 & 7.1 & 7.29 & $7.3 \pm 0.1$ & $6.2 \pm 0.3$ & $1.4 \pm 0.1$ & $9.07 \pm 0.59$ & $0.01 \pm 0.01$ \\
\hline & $\mathrm{C}^{18} \mathrm{O}$ & $3-2$ & 0.22 & 7.3 & 3.85 & $7.3 \pm 0.1$ & $3.6 \pm 0.4$ & $0.8 \pm 0.1$ & $3.11 \pm 0.50$ & $0.00 \pm 0.01$ \\
\hline
\end{tabular}

Notes. ${ }^{(a)}$ From Gaussian fits. ${ }^{(b)}$ Calculated by integrating over $v_{\mathrm{LSR}} \pm 3$ FWHM. ${ }^{(c)}$ Calculated using Eq. (1) from Mardones et al. (1997). ${ }^{(d)}$ Central absorption masked in Gaussian fit. ${ }^{(e)}$ Position of maximum of optically thick line used due to non-Gaussian line shape. ${ }^{(f)}$ Resampled to $0.3 \mathrm{~km} \mathrm{~s}^{-1}$ to fit weak line. 
J. C. Mottram et al.: Outflows, infall and evolution of a sample of embedded low-mass protostars

Table A.9. continued.

\begin{tabular}{|c|c|c|c|c|c|c|c|c|c|c|}
\hline Name & Molecule & Transition & $\begin{array}{c}\sigma_{\text {rms }} \\
(\mathrm{K})\end{array}$ & $\begin{array}{c}v_{\max } \\
\left(\mathrm{km} \mathrm{s}^{-1}\right)\end{array}$ & $\begin{array}{l}T_{\max } \\
(\mathrm{K})\end{array}$ & $\begin{array}{c}v_{\text {peak }}{ }^{a} \\
\left(\mathrm{~km} \mathrm{~s}^{-1}\right)\end{array}$ & $\begin{array}{c}T_{\text {peak }}{ }^{a} \\
(\mathrm{~K})\end{array}$ & $\begin{array}{c}F W H M^{a} \\
\left(\mathrm{~km} \mathrm{~s}^{-1}\right)\end{array}$ & $\begin{array}{l}\int T_{\mathrm{MB}} \mathrm{d} v^{b} \\
\left(\mathrm{~K} \mathrm{~km} \mathrm{~s}^{-1}\right)\end{array}$ & $\begin{array}{c}\delta v^{c} \\
\left(\mathrm{~km} \mathrm{~s}^{-1}\right)\end{array}$ \\
\hline & $\mathrm{HCO}^{+}$ & $4-3$ & 0.15 & 7.1 & 5.54 & $7.3 \pm 0.1$ & $4.1 \pm 0.2$ & $1.3 \pm 0.1$ & $5.70 \pm 0.40$ & $-0.01 \pm 0.01$ \\
\hline & $\mathrm{H}^{13} \mathrm{CO}^{+}$ & $4-3$ & 0.13 & 7.5 & 0.70 & $7.4 \pm 0.1$ & $0.7 \pm 0.2$ & $0.9 \pm 0.3$ & $0.65 \pm 0.31$ & $0.00 \pm 0.01$ \\
\hline \multirow[t]{4}{*}{ PER 06} & ${ }^{13} \mathrm{CO}$ & $3-2$ & 0.16 & 6.6 & 8.39 & $7.2 \pm 0.1$ & $6.8 \pm 0.1$ & $2.8 \pm 0.1$ & $20.58 \pm 0.55$ & $-0.08 \pm 0.01$ \\
\hline & $\mathrm{C}^{18} \mathrm{O}$ & $3-2$ & 0.15 & 7.0 & 3.46 & $7.3 \pm 0.1$ & $3.2 \pm 0.1$ & $2.0 \pm 0.1$ & $6.84 \pm 0.36$ & $0.00 \pm 0.01$ \\
\hline & $\mathrm{HCO}^{+}$ & $4-3$ & 0.20 & 7.0 & 5.44 & $7.0 \pm 0.1$ & $5.0 \pm 0.2$ & $1.4 \pm 0.1$ & $7.33 \pm 0.47$ & $-0.18 \pm 0.01$ \\
\hline & $\mathrm{H}^{13} \mathrm{CO}^{+}$ & $4-3$ & 0.11 & 6.8 & 0.44 & $7.0 \pm 0.2$ & $0.4 \pm 0.2$ & $0.8 \pm 0.4$ & $0.36 \pm 0.26$ & $0.00 \pm 0.01$ \\
\hline \multirow[t]{4}{*}{ PER 07} & ${ }^{13} \mathrm{CO}$ & $3-2$ & 0.21 & 7.3 & 5.34 & $7.2 \pm 0.1$ & $4.6 \pm 0.1$ & $2.2 \pm 0.1$ & $10.93 \pm 0.51$ & $-0.12 \pm 0.04$ \\
\hline & $\mathrm{C}^{18} \mathrm{O}$ & $3-2$ & 0.23 & 7.3 & 1.08 & $7.4 \pm 0.2$ & $1.0 \pm 0.3$ & $1.3 \pm 0.4$ & $1.31 \pm 0.53$ & $0.00 \pm 0.01$ \\
\hline & $\mathrm{HCO}^{+}$ & $4-3$ & 0.14 & 7.3 & 2.63 & $7.1 \pm 0.1$ & $2.6 \pm 0.2$ & $1.1 \pm 0.1$ & $2.96 \pm 0.32$ & $-0.17 \pm 0.05$ \\
\hline & $\mathrm{H}^{13} \mathrm{CO}^{+}$ & $4-3$ & 0.19 & - & $<0.58$ & - & - & - & - & - \\
\hline \multirow[t]{4}{*}{ PER 08} & ${ }^{13} \mathrm{CO}$ & $3-2$ & 0.34 & 7.8 & 11.17 & $7.3 \pm 0.1$ & $11.0 \pm 0.2$ & $2.9 \pm 0.1$ & $33.92 \pm 1.12$ & $-0.22 \pm 0.02$ \\
\hline & $\mathrm{C}^{18} \mathrm{O}$ & $3-2$ & 0.38 & 7.8 & 6.04 & $7.7 \pm 0.1$ & $6.1 \pm 0.4$ & $1.6 \pm 0.1$ & $10.30 \pm 1.09$ & $0.00 \pm 0.01$ \\
\hline & $\mathrm{HCO}^{+}$ & $4-3$ & 0.19 & 8.4 & 8.63 & $8.1 \pm 0.1$ & $6.9 \pm 0.2$ & $1.7 \pm 0.1$ & $12.31 \pm 0.60$ & $0.29 \pm 0.02$ \\
\hline & $\mathrm{H}^{13} \mathrm{CO}^{+}$ & $4-3$ & 0.10 & 8.2 & 1.20 & $8.3 \pm 0.1$ & $1.2 \pm 0.2$ & $0.7 \pm 0.1$ & $0.90 \pm 0.24$ & $0.00 \pm 0.01$ \\
\hline \multirow{4}{*}{ PER 09} & ${ }^{13} \mathrm{CO}$ & $3-2$ & 0.31 & 6.9 & 20.45 & $7.2 \pm 0.1$ & $19.1 \pm 0.4$ & $2.0 \pm 0.1$ & $39.84 \pm 1.20$ & $-0.24 \pm 0.02$ \\
\hline & $\mathrm{C}^{18} \mathrm{O}$ & $3-2$ & 0.31 & 7.5 & 6.33 & $7.5 \pm 0.1$ & $6.4 \pm 0.5$ & $1.2 \pm 0.1$ & $8.28 \pm 0.89$ & $0.00 \pm 0.01$ \\
\hline & $\mathrm{HCO}^{+}$ & $4-3$ & 0.19 & 7.6 & 1.50 & $7.5 \pm 0.1$ & $1.4 \pm 0.2$ & $1.6 \pm 0.2$ & $2.23 \pm 0.44$ & $-0.03 \pm 0.01$ \\
\hline & $\mathrm{H}^{13} \mathrm{CO}^{+}$ & $4-3$ & 0.11 & - & $<0.34$ & - & - & - & - & - \\
\hline \multirow{4}{*}{ PER 10} & ${ }^{13} \mathrm{CO}$ & $3-2$ & 0.23 & 8.1 & 8.26 & $8.1 \pm 0.1$ & $7.5 \pm 0.1$ & $3.0 \pm 0.1$ & $23.67 \pm 0.58$ & $-0.38 \pm 0.03$ \\
\hline & $\mathrm{C}^{18} \mathrm{O}$ & $3-2$ & 0.20 & 8.7 & 3.53 & $8.7 \pm 0.1$ & $3.1 \pm 0.2$ & $1.4 \pm 0.1$ & $4.50 \pm 0.48$ & $0.00 \pm 0.01$ \\
\hline & $\mathrm{HCO}^{+}$ & $4-3$ & 0.30 & 8.5 & 3.94 & $8.5 \pm 0.1$ & $4.0 \pm 0.3$ & $1.4 \pm 0.1$ & $5.89 \pm 0.71$ & $-0.08 \pm 0.01$ \\
\hline & $\mathrm{H}^{13} \mathrm{CO}^{+}$ & $4-3$ & 0.18 & 9.1 & 0.89 & $9.0 \pm 0.1$ & $0.9 \pm 0.4$ & $0.7 \pm 0.3$ & $0.63 \pm 0.42$ & $0.00 \pm 0.01$ \\
\hline \multirow[t]{4}{*}{ PER 12} & ${ }^{13} \mathrm{CO}$ & $3-2$ & 0.28 & 7.8 & 5.45 & $7.8 \pm 0.1$ & $5.1 \pm 0.2$ & $2.0 \pm 0.1$ & $10.89 \pm 0.80$ & $0.05 \pm 0.01$ \\
\hline & $\mathrm{C}^{18} \mathrm{O}$ & $3-2$ & 0.31 & 7.5 & 3.05 & $7.8 \pm 0.1$ & $3.1 \pm 0.5$ & $1.0 \pm 0.2$ & $3.24 \pm 0.88$ & $0.00 \pm 0.01$ \\
\hline & $\mathrm{HCO}^{+}$ & $4-3$ & 0.21 & 7.4 & 3.33 & $7.6 \pm 0.1$ & $2.3 \pm 0.2$ & $1.4 \pm 0.2$ & $3.47 \pm 0.52$ & $-0.18 \pm 0.04$ \\
\hline & $\mathrm{H}^{13} \mathrm{CO}^{+}$ & $4-3$ & 0.13 & 7.8 & 0.59 & $8.0 \pm 0.2$ & $0.6 \pm 0.3$ & $0.7 \pm 0.4$ & $0.43 \pm 0.30$ & $0.00 \pm 0.01$ \\
\hline \multirow[t]{3}{*}{ PER 13} & $\mathrm{C}^{18} \mathrm{O}$ & $3-2$ & 0.35 & 7.9 & 2.19 & $8.0 \pm 0.1$ & $2.4 \pm 0.8$ & $0.6 \pm 0.2$ & $1.57 \pm 0.82$ & $0.00 \pm 0.01$ \\
\hline & $\mathrm{HCO}^{+}$ & $4-3$ & 0.11 & 7.9 & 4.70 & $7.9 \pm 0.1$ & $4.2 \pm 0.2$ & $1.0 \pm 0.1$ & $4.44 \pm 0.30$ & $-0.18 \pm 0.07$ \\
\hline & $\mathrm{H}^{13} \mathrm{CO}^{+}$ & $4-3$ & 0.17 & 8.1 & 1.42 & $8.1 \pm 0.1$ & $1.3 \pm 0.3$ & $0.7 \pm 0.2$ & $0.94 \pm 0.40$ & $0.00 \pm 0.01$ \\
\hline \multirow[t]{4}{*}{ PER 14} & ${ }^{13} \mathrm{CO}$ & $3-2$ & 0.28 & 6.0 & 5.09 & $6.0 \pm 0.1$ & $4.4 \pm 0.4$ & $1.1 \pm 0.1$ & $5.12 \pm 0.67$ & $-0.34 \pm 0.20$ \\
\hline & $\mathrm{C}^{18} \mathrm{O}$ & $3-2$ & 0.35 & 6.0 & 1.33 & $6.2 \pm 0.2$ & $1.3 \pm 0.7$ & $0.7 \pm 0.4$ & $1.01 \pm 0.82$ & $0.00 \pm 0.01$ \\
\hline & $\mathrm{HCO}^{+}$ & $4-3$ & 0.24 & 5.6 & 1.64 & $5.9 \pm 0.1$ & $1.1 \pm 0.2$ & $1.7 \pm 0.3$ & $2.10 \pm 0.56$ & $-0.43 \pm 0.26$ \\
\hline & $\mathrm{H}^{13} \mathrm{CO}^{+}$ & $4-3$ & 0.13 & - & $<0.38$ & - & - & - & - & - \\
\hline \multirow[t]{3}{*}{ PER 15} & $\mathrm{C}^{18} \mathrm{O}^{f}$ & $3-2$ & 0.30 & 6.9 & 1.20 & $6.9 \pm 0.2$ & $1.0 \pm 0.4$ & $1.4 \pm 0.6$ & $1.46 \pm 0.83$ & $0.00 \pm 0.01$ \\
\hline & $\mathrm{HCO}^{+}$ & $4-3$ & 0.12 & 6.7 & 3.52 & $7.1 \pm 0.1$ & $2.8 \pm 0.1$ & $1.4 \pm 0.1$ & $4.26 \pm 0.32$ & $0.10 \pm 0.04$ \\
\hline & $\mathrm{H}^{13} \mathrm{CO}^{+}$ & $4-3$ & 0.16 & 7.1 & 0.82 & $7.2 \pm 0.2$ & $0.6 \pm 0.3$ & $0.7 \pm 0.4$ & $0.45 \pm 0.38$ & $0.00 \pm 0.01$ \\
\hline \multirow[t]{3}{*}{ PER 16} & $\mathrm{C}^{18} \mathrm{O}$ & $3-2$ & 0.41 & 7.1 & 2.54 & $7.0 \pm 0.1$ & $2.2 \pm 0.5$ & $1.2 \pm 0.3$ & $2.87 \pm 0.94$ & $0.00 \pm 0.01$ \\
\hline & $\mathrm{HCO}^{+}$ & $4-3$ & 0.15 & 6.5 & 4.17 & $6.6 \pm 0.1$ & $3.9 \pm 0.3$ & $0.9 \pm 0.1$ & $3.70 \pm 0.38$ & $-0.29 \pm 0.07$ \\
\hline & $\mathrm{H}^{13} \mathrm{CO}^{+}$ & $4-3$ & 0.16 & 7.3 & 0.56 & $6.9 \pm 0.2$ & $0.5 \pm 0.2$ & $1.0 \pm 0.5$ & $0.56 \pm 0.37$ & $0.00 \pm 0.01$ \\
\hline \multirow[t]{4}{*}{ PER 17} & ${ }^{13} \mathrm{CO}$ & $3-2$ & 0.19 & 6.0 & 3.68 & $6.5 \pm 0.1$ & $3.4 \pm 0.1$ & $2.3 \pm 0.1$ & $8.31 \pm 0.47$ & $-0.09 \pm 0.02$ \\
\hline & $\mathrm{C}^{18} \mathrm{O}$ & $3-2$ & 0.32 & 6.4 & 2.01 & $6.6 \pm 0.1$ & $1.9 \pm 0.3$ & $1.4 \pm 0.3$ & $2.69 \pm 0.74$ & $0.00 \pm 0.01$ \\
\hline & $\mathrm{HCO}^{+}$ & $4-3$ & 0.15 & 6.0 & 1.84 & $6.0 \pm 0.1$ & $1.6 \pm 0.3$ & $0.8 \pm 0.2$ & $1.39 \pm 0.35$ & $-0.44 \pm 0.09$ \\
\hline & $\mathrm{H}^{13} \mathrm{CO}^{+}$ & $4-3$ & 0.11 & - & $<0.32$ & - & - & - & - & - \\
\hline \multirow[t]{3}{*}{ PER 18} & ${ }^{13} \mathrm{CO}$ & $3-2$ & 0.18 & 6.7 & 3.86 & $6.6 \pm 0.1$ & $4.1 \pm 0.1$ & $1.7 \pm 0.1$ & $7.42 \pm 0.41$ & $-0.03 \pm 0.01$ \\
\hline & $\mathrm{C}^{18} \mathrm{O}$ & $3-2$ & 0.20 & 6.7 & 2.60 & $6.6 \pm 0.1$ & $2.7 \pm 0.3$ & $0.9 \pm 0.1$ & $2.55 \pm 0.47$ & $0.00 \pm 0.01$ \\
\hline & $\mathrm{HCO}^{+}$ & $4-3$ & 0.13 & 6.2 & 1.45 & $6.4 \pm 0.1$ & $1.0 \pm 0.1$ & $1.6 \pm 0.2$ & $1.67 \pm 0.29$ & $-0.28 \pm 0.04$ \\
\hline PER 19 & $\mathrm{C}^{18} \mathrm{O}$ & $3-2$ & 0.51 & 6.9 & 2.17 & $6.8 \pm 0.2$ & $1.8 \pm 1.0$ & $0.7 \pm 0.5$ & $1.45 \pm 1.19$ & $0.00 \pm 0.01$ \\
\hline & $\mathrm{HCO}^{+}$ & $4-3$ & 0.18 & 6.7 & 1.80 & $6.9 \pm 0.1$ & $1.5 \pm 0.2$ & $1.0 \pm 0.2$ & $1.67 \pm 0.41$ & $0.22 \pm 0.14$ \\
\hline & $\mathrm{H}^{13} \mathrm{CO}^{+}$ & $4-3$ & 0.15 & - & $<0.46$ & - & - & - & - & - \\
\hline PER 20 & ${ }^{13} \mathrm{CO}$ & $3-2$ & 0.28 & 8.7 & 9.61 & $8.9 \pm 0.1$ & $10.0 \pm 0.3$ & $1.5 \pm 0.1$ & $16.13 \pm 0.78$ & $-0.01 \pm 0.01$ \\
\hline & $\mathrm{C}^{18} \mathrm{O}$ & $3-2$ & 0.38 & 9.0 & 5.46 & $8.9 \pm 0.1$ & $5.5 \pm 0.8$ & $0.8 \pm 0.1$ & $4.72 \pm 1.08$ & $0.00 \pm 0.01$ \\
\hline & $\mathrm{HCO}^{+}$ & $4-3$ & 0.39 & 9.3 & 3.42 & $8.9 \pm 0.1$ & $2.8 \pm 0.3$ & $2.0 \pm 0.2$ & $5.93 \pm 0.93$ & $0.01 \pm 0.01$ \\
\hline & $\mathrm{H}^{13} \mathrm{CO}^{+}$ & $4-3$ & 0.16 & 9.3 & 1.09 & $9.2 \pm 0.1$ & $1.1 \pm 0.3$ & $0.7 \pm 0.2$ & $0.76 \pm 0.37$ & $0.00 \pm 0.01$ \\
\hline PER 21 & ${ }^{13} \mathrm{CO}$ & $3-2$ & 0.21 & 8.7 & 10.32 & $8.6 \pm 0.1$ & $10.4 \pm 0.2$ & $1.7 \pm 0.1$ & $19.13 \pm 0.56$ & $-0.12 \pm 0.01$ \\
\hline & $\mathrm{C}^{18} \mathrm{O}$ & $3-2$ & 0.28 & 8.9 & 5.00 & $8.8 \pm 0.1$ & $4.6 \pm 0.4$ & $0.9 \pm 0.1$ & $4.51 \pm 0.67$ & $0.00 \pm 0.01$ \\
\hline & $\mathrm{HCO}^{+}$ & $4-3$ & 0.14 & 8.7 & 3.90 & $8.8 \pm 0.1$ & $3.8 \pm 0.2$ & $1.0 \pm 0.1$ & $4.01 \pm 0.33$ & $0.02 \pm 0.01$ \\
\hline & $\mathrm{H}^{13} \mathrm{CO}^{+}$ & $4-3$ & 0.15 & 9.1 & 0.71 & $9.0 \pm 0.1$ & $0.8 \pm 0.4$ & $0.5 \pm 0.3$ & $0.42 \pm 0.35$ & $0.00 \pm 0.01$ \\
\hline PER 22 & ${ }^{13} \mathrm{CO}$ & $3-2$ & 0.21 & 9.9 & 8.59 & $9.7 \pm 0.1$ & $8.5 \pm 0.1$ & $2.5 \pm 0.1$ & $22.67 \pm 0.57$ & $-0.06 \pm 0.01$ \\
\hline & $\mathrm{C}^{18} \mathrm{O}$ & $3-2$ & 0.24 & 9.7 & 3.92 & $9.8 \pm 0.1$ & $3.3 \pm 0.2$ & $1.5 \pm 0.1$ & $5.30 \pm 0.55$ & $0.00 \pm 0.01$ \\
\hline & $\mathrm{HCO}^{+}$ & $4-3$ & 0.38 & 9.7 & 5.80 & $10.2 \pm 0.1$ & $4.3 \pm 0.2$ & $2.8 \pm 0.1$ & $12.72 \pm 0.91$ & $0.24 \pm 0.02$ \\
\hline & $\mathrm{H}^{13} \mathrm{CO}^{+}$ & $4-3$ & 0.15 & - & $<0.46$ & - & - & - & - & - \\
\hline SCO 01 & ${ }^{13} \mathrm{CO}$ & $3-2$ & 0.23 & 3.6 & 4.02 & $3.6 \pm 0.1$ & $3.5 \pm 0.3$ & $1.0 \pm 0.1$ & $3.61 \pm 0.53$ & $0.00 \pm 0.01$ \\
\hline
\end{tabular}


Table A.9. continued.

\begin{tabular}{|c|c|c|c|c|c|c|c|c|c|c|}
\hline Name & Molecule & Transition & $\begin{array}{c}\sigma_{\mathrm{rms}} \\
(\mathrm{K})\end{array}$ & $\begin{array}{c}v_{\max } \\
\left(\mathrm{km} \mathrm{s}^{-1}\right)\end{array}$ & $\begin{array}{l}T_{\max } \\
(\mathrm{K})\end{array}$ & $\begin{array}{c}v_{\text {peak }}{ }^{a} \\
\left(\mathrm{~km} \mathrm{~s}^{-1}\right) \\
\end{array}$ & $\begin{array}{c}T_{\text {peak }}{ }^{a} \\
(\mathrm{~K}) \\
\end{array}$ & $\begin{array}{l}F W H M^{a} \\
\left(\mathrm{~km} \mathrm{~s}^{-1}\right)\end{array}$ & $\begin{array}{l}\int T_{\mathrm{MB}} \mathrm{d} v^{b} \\
\left(\mathrm{Kkm} \mathrm{s}^{-1}\right)\end{array}$ & $\begin{array}{c}\delta v^{c} \\
\left(\mathrm{~km} \mathrm{~s}^{-1}\right) \\
\end{array}$ \\
\hline & $\mathrm{C}^{18} \mathrm{O}$ & $3-2$ & 0.26 & - & $<0.79$ & - & - & - & - & - \\
\hline & $\mathrm{HCO}^{+}$ & $4-3$ & 0.30 & - & $<0.90$ & - & - & - & - & - \\
\hline & $\mathrm{H}^{13} \mathrm{CO}^{+}$ & $4-3$ & 0.22 & - & $<0.65$ & - & - & - & - & - \\
\hline \multirow[t]{3}{*}{ SERS 01} & ${ }^{13} \mathrm{CO}$ & $3-2$ & 0.23 & 7.4 & 4.55 & $8.1 \pm 0.1$ & $3.7 \pm 0.2$ & $2.4 \pm 0.1$ & $9.25 \pm 0.63$ & $-0.02 \pm 0.01$ \\
\hline & $\mathrm{C}^{18} \mathrm{O}$ & $3-2$ & 0.30 & 8.2 & 1.88 & $8.2 \pm 0.1$ & $1.4 \pm 0.3$ & $1.6 \pm 0.3$ & $2.45 \pm 0.68$ & $0.00 \pm 0.01$ \\
\hline & $\mathrm{HCO}^{+}$ & $4-3$ & 0.36 & 8.6 & 4.07 & $8.5 \pm 0.1$ & $3.3 \pm 0.3$ & $1.8 \pm 0.2$ & $6.30 \pm 0.85$ & $0.26 \pm 0.06^{e}$ \\
\hline \multirow[t]{3}{*}{ SERS 02} & ${ }^{13} \mathrm{CO}$ & $3-2$ & 0.26 & 7.2 & 6.65 & $8.1 \pm 0.1$ & $5.4 \pm 0.1$ & $5.0 \pm 0.1$ & $29.00 \pm 0.85$ & $0.12 \pm 0.01$ \\
\hline & $\mathrm{C}^{18} \mathrm{O}$ & $3-2$ & 0.33 & 7.8 & 4.59 & $7.8 \pm 0.1$ & $4.0 \pm 0.2$ & $2.3 \pm 0.1$ & $9.59 \pm 0.77$ & $0.00 \pm 0.01$ \\
\hline & $\mathrm{HCO}^{+d}$ & $4-3$ & 0.38 & 7.0 & 10.17 & $7.7 \pm 0.1$ & $11.5 \pm 0.4$ & $3.0 \pm 0.1$ & $36.94 \pm 1.61$ & $-0.36 \pm 0.02^{e}$ \\
\hline \multirow[t]{4}{*}{ TAU 01} & ${ }^{13} \mathrm{CO}$ & $3-2$ & 0.13 & 7.2 & 4.13 & $7.0 \pm 0.1$ & $4.0 \pm 0.1$ & $1.8 \pm 0.1$ & $7.66 \pm 0.39$ & $0.18 \pm 0.03$ \\
\hline & $\mathrm{C}^{18} \mathrm{O}$ & $3-2$ & 0.20 & 6.9 & 2.48 & $6.8 \pm 0.1$ & $2.4 \pm 0.4$ & $0.9 \pm 0.2$ & $2.31 \pm 0.55$ & $0.00 \pm 0.01$ \\
\hline & $\mathrm{HCO}^{+}$ & $4-3$ & 0.22 & 6.6 & 3.01 & $6.8 \pm 0.1$ & $2.7 \pm 0.3$ & $1.2 \pm 0.2$ & $3.49 \pm 0.65$ & $-0.01 \pm 0.01$ \\
\hline & $\mathrm{H}^{13} \mathrm{CO}^{+}$ & $4-3$ & 0.11 & 6.9 & 0.46 & $6.9 \pm 0.2$ & $0.4 \pm 0.3$ & $0.6 \pm 0.4$ & $0.26 \pm 0.28$ & $0.00 \pm 0.01$ \\
\hline \multirow[t]{3}{*}{ TAU 02} & $\mathrm{C}^{18} \mathrm{O}$ & $3-2$ & 0.29 & 6.6 & 2.54 & $6.6 \pm 0.1$ & $2.1 \pm 0.6$ & $0.7 \pm 0.2$ & $1.58 \pm 0.67$ & $0.00 \pm 0.01$ \\
\hline & $\mathrm{HCO}^{+}$ & $4-3$ & 0.34 & 7.2 & 1.79 & $7.2 \pm 0.1$ & $1.7 \pm 0.9$ & $0.5 \pm 0.3$ & $0.95 \pm 0.79$ & $0.85 \pm 0.28$ \\
\hline & $\mathrm{H}^{13} \mathrm{CO}^{+}$ & $4-3$ & 0.15 & - & $<0.45$ & - & - & - & - & - \\
\hline \multirow{4}{*}{ TAU 03} & ${ }^{13} \mathrm{CO}$ & $3-2$ & 0.23 & 7.8 & 2.26 & $7.4 \pm 0.1$ & $1.6 \pm 0.1$ & $2.3 \pm 0.2$ & $3.84 \pm 0.55$ & $0.00 \pm 0.01$ \\
\hline & $\mathrm{C}^{18} \mathrm{O}$ & $3-2$ & 0.27 & - & $<0.82$ & - & - & - & - & - \\
\hline & $\mathrm{HCO}^{+}$ & $4-3$ & 0.29 & - & $<0.86$ & - & - & - & - & - \\
\hline & $\mathrm{H}^{13} \mathrm{CO}^{+}$ & $4-3$ & 0.13 & - & $<0.40$ & - & - & - & - & - \\
\hline \multirow[t]{4}{*}{ TAU 04} & ${ }^{13} \mathrm{CO}$ & $3-2$ & 0.24 & 6.6 & 5.29 & $6.5 \pm 0.1$ & $4.1 \pm 0.2$ & $1.7 \pm 0.1$ & $7.39 \pm 0.58$ & $0.16 \pm 0.06$ \\
\hline & $\mathrm{C}^{18} \mathrm{O}$ & $3-2$ & 0.31 & 6.2 & 2.19 & $6.3 \pm 0.1$ & $2.1 \pm 0.6$ & $0.7 \pm 0.2$ & $1.54 \pm 0.72$ & $0.00 \pm 0.01$ \\
\hline & $\mathrm{HCO}^{+}$ & $4-3$ & 0.28 & - & $<0.84$ & - & - & - & - & - \\
\hline & $\mathrm{H}^{13} \mathrm{CO}^{+}$ & $4-3$ & 0.15 & - & $<0.44$ & - & - & - & - & - \\
\hline \multirow[t]{2}{*}{ TAU 06} & $\mathrm{C}^{18} \mathrm{O}$ & $3-2$ & 0.32 & 7.2 & 1.53 & $7.2 \pm 0.2$ & $1.3 \pm 0.3$ & $1.4 \pm 0.4$ & $1.93 \pm 0.73$ & $0.00 \pm 0.01$ \\
\hline & $\mathrm{HCO}^{+}$ & $4-3$ & 0.28 & 7.4 & 2.66 & $7.4 \pm 0.1$ & $2.7 \pm 0.5$ & $0.8 \pm 0.2$ & $2.29 \pm 0.66$ & $0.12 \pm 0.03$ \\
\hline \multirow[t]{4}{*}{ TAU 07} & ${ }^{13} \mathrm{CO}$ & $3-2$ & 0.21 & 5.8 & 1.70 & $6.3 \pm 0.1$ & $1.5 \pm 0.2$ & $2.0 \pm 0.2$ & $3.11 \pm 0.49$ & $0.00 \pm 0.01$ \\
\hline & $\mathrm{C}^{18} \mathrm{O}$ & $3-2$ & 0.26 & - & $<0.78$ & - & - & - & - & - \\
\hline & $\mathrm{HCO}^{+}$ & $4-3$ & 0.27 & - & $<0.82$ & - & - & - & - & - \\
\hline & $\mathrm{H}^{13} \mathrm{CO}^{+}$ & $4-3$ & 0.18 & - & $<0.54$ & - & - & - & - & - \\
\hline \multirow[t]{3}{*}{ TAU 08} & $\mathrm{C}^{18} \mathrm{O}$ & $3-2$ & 0.80 & - & $<2.41$ & - & - & - & - & - \\
\hline & $\mathrm{HCO}^{+}$ & $4-3$ & 0.25 & - & $<0.75$ & - & - & - & - & - \\
\hline & $\mathrm{H}^{13} \mathrm{CO}^{+}$ & $4-3$ & 0.17 & - & $<0.50$ & - & - & - & - & - \\
\hline \multirow{3}{*}{ TAU 09} & $\mathrm{C}^{18} \mathrm{O}$ & $3-2$ & 0.48 & 5.3 & 2.63 & $5.5 \pm 0.1$ & $2.4 \pm 1.0$ & $0.7 \pm 0.3$ & $1.76 \pm 1.10$ & $0.00 \pm 0.01$ \\
\hline & $\mathrm{HCO}^{+}$ & $4-3$ & 0.21 & 5.3 & 1.27 & $5.3 \pm 0.1$ & $1.0 \pm 0.2$ & $1.8 \pm 0.3$ & $1.89 \pm 0.47$ & $-0.25 \pm 0.12^{e}$ \\
\hline & $\mathrm{H}^{13} \mathrm{CO}^{+}$ & $4-3$ & 0.11 & - & $<0.32$ & - & - & - & - & - \\
\hline \multirow[t]{4}{*}{ W40 01} & ${ }^{13} \mathrm{CO}$ & $3-2$ & 0.26 & 5.2 & 15.10 & $5.2 \pm 0.1$ & $16.1 \pm 0.2$ & $1.9 \pm 0.1$ & $32.16 \pm 0.75$ & $0.22 \pm 0.01$ \\
\hline & $\mathrm{C}^{18} \mathrm{O}$ & $3-2$ & 0.36 & 4.8 & 6.65 & $4.9 \pm 0.1$ & $6.8 \pm 0.4$ & $1.3 \pm 0.1$ & $9.36 \pm 0.82$ & $0.00 \pm 0.01$ \\
\hline & $\mathrm{HCO}^{+}$ & $4-3$ & 0.41 & 4.4 & 4.52 & $4.6 \pm 0.1$ & $4.5 \pm 0.5$ & $1.2 \pm 0.2$ & $5.78 \pm 0.97$ & $-0.23 \pm 0.02$ \\
\hline & $\mathrm{H}^{13} \mathrm{CO}^{+}$ & $4-3$ & 0.14 & 4.6 & 0.84 & $4.7 \pm 0.1$ & $0.7 \pm 0.3$ & $0.8 \pm 0.3$ & $0.58 \pm 0.33$ & $0.00 \pm 0.01$ \\
\hline \multirow[t]{4}{*}{ W40 02} & ${ }^{13} \mathrm{CO}$ & $3-2$ & 0.30 & 4.8 & 27.47 & $4.8 \pm 0.1$ & $27.2 \pm 0.4$ & $1.1 \pm 0.1$ & $31.95 \pm 0.75$ & $0.01 \pm 0.01$ \\
\hline & $\mathrm{C}^{18} \mathrm{O}$ & $3-2$ & 0.37 & 4.8 & 6.90 & $4.8 \pm 0.1$ & $6.8 \pm 0.6$ & $0.9 \pm 0.1$ & $6.85 \pm 0.87$ & $0.00 \pm 0.01$ \\
\hline & $\mathrm{HCO}^{+}$ & $4-3$ & 0.49 & 4.8 & 3.74 & $4.9 \pm 0.1$ & $2.7 \pm 0.4$ & $1.8 \pm 0.3$ & $5.18 \pm 1.15$ & $0.10 \pm 0.01$ \\
\hline & $\mathrm{H}^{13} \mathrm{CO}^{+}$ & $4-3$ & 0.15 & 5.0 & 0.63 & $5.1 \pm 0.2$ & $0.5 \pm 0.3$ & $0.8 \pm 0.5$ & $0.42 \pm 0.35$ & $0.00 \pm 0.01$ \\
\hline W40 03 & ${ }^{13} \mathrm{CO}$ & $3-2$ & 0.29 & 5.8 & 19.39 & $6.1 \pm 0.1$ & $12.0 \pm 0.4$ & $2.9 \pm 0.1$ & $36.48 \pm 1.89$ & $-0.20 \pm 0.01$ \\
\hline & $\mathrm{C}^{18} \mathrm{O}$ & $3-2$ & 0.34 & 6.2 & 12.97 & $6.4 \pm 0.1$ & $12.1 \pm 0.4$ & $1.4 \pm 0.1$ & $17.45 \pm 0.91$ & $0.00 \pm 0.01$ \\
\hline & $\mathrm{HCO}^{+}$ & $4-3$ & 0.48 & 6.6 & 6.55 & $6.6 \pm 0.1$ & $6.6 \pm 0.4$ & $1.6 \pm 0.1$ & $11.43 \pm 1.10$ & $0.17 \pm 0.01$ \\
\hline W40 04 & ${ }^{13} \mathrm{CO}$ & $3-2$ & 0.29 & 6.0 & 16.20 & $7.4 \pm 0.1$ & $8.7 \pm 0.3$ & $5.6 \pm 0.2$ & $51.37 \pm 2.27$ & $0.52 \pm 0.02$ \\
\hline & $\mathrm{C}^{18} \mathrm{O}$ & $3-2$ & 0.35 & 6.6 & 13.12 & $6.7 \pm 0.1$ & $13.2 \pm 0.4$ & $1.3 \pm 0.1$ & $18.29 \pm 0.85$ & $0.00 \pm 0.01$ \\
\hline & $\mathrm{HCO}^{+}$ & $4-3$ & 0.35 & 7.0 & 2.57 & $6.8 \pm 0.1$ & $2.4 \pm 0.4$ & $1.3 \pm 0.2$ & $3.32 \pm 0.80$ & $0.09 \pm 0.01$ \\
\hline & $\mathrm{H}^{13} \mathrm{CO}^{+}$ & $4-3$ & 0.11 & - & $<0.32$ & - & - & - & - & - \\
\hline W40 05 & ${ }^{13} \mathrm{CO}$ & $3-2$ & 0.21 & 5.8 & 14.66 & $7.6 \pm 0.1$ & $11.5 \pm 0.3$ & $4.6 \pm 0.1$ & $55.71 \pm 1.92$ & $0.73 \pm 0.02$ \\
\hline & $\mathrm{C}^{18} \mathrm{O}$ & $3-2$ & 0.26 & 6.2 & 14.47 & $6.5 \pm 0.1$ & $14.6 \pm 0.3$ & $1.5 \pm 0.1$ & $23.71 \pm 0.76$ & $0.00 \pm 0.01$ \\
\hline & $\mathrm{HCO}^{+}$ & $4-3$ & 0.28 & 6.6 & 3.20 & $6.8 \pm 0.1$ & $3.0 \pm 0.3$ & $1.5 \pm 0.2$ & $4.78 \pm 0.67$ & $0.16 \pm 0.01$ \\
\hline & $\mathrm{H}^{13} \mathrm{CO}^{+}$ & $4-3$ & 0.11 & 6.2 & 0.39 & $6.5 \pm 0.2$ & $0.4 \pm 0.2$ & $0.7 \pm 0.5$ & $0.27 \pm 0.26$ & $0.00 \pm 0.01$ \\
\hline W40 06 & ${ }^{13} \mathrm{CO}$ & $3-2$ & 0.20 & 5.8 & 12.81 & $6.0 \pm 0.1$ & $11.8 \pm 0.3$ & $1.9 \pm 0.1$ & $23.49 \pm 0.91$ & $-0.29 \pm 0.03$ \\
\hline & $\mathrm{C}^{18} \mathrm{O}$ & $3-2$ & 0.23 & 7.0 & 5.54 & $6.6 \pm 0.1$ & $3.0 \pm 0.2$ & $1.9 \pm 0.2$ & $6.26 \pm 0.73$ & $0.00 \pm 0.01$ \\
\hline & $\mathrm{HCO}^{+}$ & $4-3$ & 0.29 & 6.8 & 1.66 & $6.9 \pm 0.1$ & $1.7 \pm 0.9$ & $0.5 \pm 0.3$ & $0.91 \pm 0.68$ & $0.16 \pm 0.01$ \\
\hline & $\mathrm{H}^{13} \mathrm{CO}^{+}$ & $4-3$ & 0.12 & - & $<0.36$ & - & - & - & - & - \\
\hline W40 07 & ${ }^{13} \mathrm{CO}$ & $3-2$ & 0.20 & 6.8 & 2.25 & $7.1 \pm 0.1$ & $1.7 \pm 0.1$ & $2.2 \pm 0.2$ & $4.00 \pm 0.47$ & $-0.38 \pm 0.07$ \\
\hline & $\mathrm{C}^{18} \mathrm{O}$ & $3-2$ & 0.25 & 7.4 & 3.34 & $7.4 \pm 0.1$ & $3.3 \pm 0.5$ & $0.7 \pm 0.1$ & $2.49 \pm 0.57$ & $0.00 \pm 0.01$ \\
\hline & $\mathrm{HCO}^{+}$ & $4-3$ & 0.28 & 7.4 & 1.51 & $7.4 \pm 0.1$ & $1.6 \pm 0.5$ & $0.9 \pm 0.3$ & $1.45 \pm 0.65$ & $0.06 \pm 0.01$ \\
\hline & $\mathrm{H}^{13} \mathrm{CO}^{+}$ & $4-3$ & 0.12 & - & $<0.35$ & - & - & - & - & - \\
\hline
\end{tabular}


Table A.10. Source evolution.

\begin{tabular}{|c|c|c|c|c|c|c|}
\hline Name & Outflow & $\mathrm{H}_{2} \mathrm{O}^{a}$ & mid- $J \mathrm{CO}^{a}$ & Stage $^{b}$ & $T_{\text {bol }}$ Class & Final Class ${ }^{c}$ \\
\hline AQU 01 & $\mathrm{Y}$ & B & B & $0 / \mathrm{I}$ & 0 & 0 \\
\hline AQU 02 & $\mathrm{Y}$ & B & B & $0 / \mathrm{I}$ & 0 & 0 \\
\hline AQU 03 & $\mathrm{~N}$ & $\mathrm{E}$ & $\mathrm{E}$ & II & I & II \\
\hline AQU 04 & $\mathrm{Y}$ & $\mathrm{E}$ & $\mathrm{N}$ & $0 / \mathrm{I}$ & I & $\mathrm{I}$ \\
\hline AQU 05 & $\mathrm{Y}$ & $\mathrm{B}$ & $\mathrm{B}$ & $0 / \mathrm{I}$ & 0 & 0 \\
\hline AQU 06 & $\mathrm{Y}$ & $\mathrm{E}$ & $\mathrm{N}$ & $0 / \mathrm{I}$ & 0 & 0 \\
\hline CHA 01 & $\mathrm{Y}$ & $\mathrm{E}$ & $\mathrm{E}$ & II & I & $\mathrm{II}^{d}$ \\
\hline CHA 02 & $\mathrm{~N}$ & $\mathrm{~N}$ & $\mathrm{~N}$ & $0 / \mathrm{I}$ & I & I \\
\hline CRA 01 & $\mathrm{Y}$ & B & $\mathrm{E}$ & $0 / \mathrm{I}$ & 0 & 0 \\
\hline ОРН 01 & $\mathrm{~N}$ & $\mathrm{E}$ & $\mathrm{E}$ & II & I & $\mathrm{II}+\mathrm{PDR} ?^{d}$ \\
\hline OPH 02 & $\mathrm{Y}$ & B & B & $0 / \mathrm{I}$ & I & I \\
\hline PER 01 & $\mathrm{Y}$ & $\mathrm{B}$ & $\mathrm{B}$ & $0 / \mathrm{I}$ & 0 & 0 \\
\hline PER 02 & $\mathrm{Y}$ & B & B & $0 / \mathrm{I}$ & 0 & 0 \\
\hline PER 04 & $\mathrm{Y}$ & $\mathrm{N}$ & $\mathrm{E}$ & $0 / \mathrm{I}$ & 0 & 0 \\
\hline PER 05 & $\mathrm{Y}$ & B & $\mathrm{E}$ & $0 / \mathrm{I}$ & I & I \\
\hline PER 06 & $\mathrm{Y}$ & B & B & $0 / \mathrm{I}$ & I & I \\
\hline PER 07 & $\mathrm{Y}$ & B & $\mathrm{N}$ & $0 / \mathrm{I}$ & 0 & 0 \\
\hline PER 08 & $\mathrm{Y}$ & B & B & $\mathrm{C}$ & I & I \\
\hline PER 09 & $\mathrm{Y}$ & B & B & $\mathrm{C}$ & I & I \\
\hline PER 10 & $\mathrm{Y}$ & B & B & $0 / \mathrm{I}$ & 0 & 0 \\
\hline PER 12 & $\mathrm{~N}$ & B & $\mathrm{E}$ & $0 / \mathrm{I}$ & 0 & 0 \\
\hline PER 13 & $\mathrm{Y}$ & B & $\mathrm{E}$ & $0 / \mathrm{I}$ & 0 & 0 \\
\hline PER 14 & $\mathrm{Y}$ & B & B & $0 / \mathrm{I}$ & I & I \\
\hline PER 15 & $\mathrm{Y}$ & B & $\mathrm{N}$ & $0 / \mathrm{I}$ & 0 & 0 \\
\hline PER 16 & $\mathrm{Y}$ & $\mathrm{E}$ & $\mathrm{E}$ & $0 / \mathrm{I}$ & 0 & 0 \\
\hline PER 17 & $\mathrm{Y}$ & B & $\mathrm{E}$ & $\mathrm{C}$ & I & I \\
\hline PER 18 & $\mathrm{Y}$ & B & B & $0 / \mathrm{I}$ & 0 & 0 \\
\hline PER 19 & Y & B & B & $0 / \mathrm{I}$ & I & I \\
\hline PER 20 & $\mathrm{Y}$ & B & B & 0/I & 0 & 0 \\
\hline PER 21 & Y & B & B & $0 / \mathrm{I}$ & 0 & 0 \\
\hline PER 22 & Y & B & B & $0 / \mathrm{I}$ & 0 & 0 \\
\hline SCO 01 & $\mathrm{~N}$ & $\mathrm{~N}$ & $\mathrm{E}$ & II & I & II \\
\hline SERS 01 & $\mathrm{Y}$ & B & B & $0 / \mathrm{I}$ & 0 & 0 \\
\hline SERS 02 & $\mathrm{Y}$ & B & B & $0 / \mathrm{I}$ & 0 & 0 \\
\hline TAU 01 & $\mathrm{Y}$ & B & $\mathrm{E}$ & $0 / \mathrm{I}$ & I & I \\
\hline TAU 02 & $\mathrm{Y}$ & B & $\mathrm{E}$ & $\mathrm{C}$ & I & I \\
\hline TAU 03 & $\mathrm{Y}$ & B & $\mathrm{N}$ & II & I & $\mathrm{II}^{d}$ \\
\hline TAU 04 & $\mathrm{Y}$ & B & B & $0 / \mathrm{I}$ & I & I \\
\hline TAU 06 & $\mathrm{Y}$ & B & $\mathrm{E}$ & $0 / \mathrm{I}$ & I & I \\
\hline TAU 07 & $\mathrm{~N}$ & $\mathrm{E}$ & $\mathrm{E}$ & II & I & II \\
\hline TAU 08 & $\mathrm{~N}$ & $\mathrm{~N}$ & $\mathrm{E}$ & II & I & II \\
\hline TAU 09 & $\mathrm{~N}$ & E & $\mathrm{E}$ & II & I & II \\
\hline W40 01 & $\mathrm{~N}$ & $\mathrm{~B}+\mathrm{P}$ & $\mathrm{P}$ & $\mathrm{C}$ & 0 & $0+\mathrm{PDR}^{d}$ \\
\hline W4002 & Y & B & B & $0 / \mathrm{I}$ & 0 & 0 \\
\hline W4003 & $\mathrm{N}$ & $\mathrm{P}$ & $\mathrm{P}$ & $\mathrm{C}$ & 0 & $\mathrm{PS}^{2}+\mathrm{PDR}^{d}$ \\
\hline W40 04 & $\mathrm{~N}$ & $\mathrm{P}$ & $\mathrm{P}$ & $\mathrm{C}$ & 0 & $\mathrm{PS}^{2}+\mathrm{PDR}^{d}$ \\
\hline W40 05 & $\mathrm{~N}$ & $\mathrm{P}$ & $\mathrm{P}$ & $\mathrm{C}$ & 0 & $\mathrm{PS}^{2}+\mathrm{PDR}^{d}$ \\
\hline W4006 & $\mathrm{N}$ & $\mathrm{N}$ & $\mathrm{P}$ & $\mathrm{C}$ & 0 & $\mathrm{PS}^{2}+\mathrm{PDR}^{d}$ \\
\hline W40 07 & Y & $\mathrm{N}$ & $\mathrm{E}$ & $\mathrm{C}$ & 0 & 0 \\
\hline
\end{tabular}

Notes. ${ }^{(a)}$ From HIFI observations: $\mathrm{B}=$ broad, $\mathrm{E}=$ envelope, $\mathrm{P}=\mathrm{PDR}$ and $\mathrm{N}=$ non-detection. ${ }^{(b)}$ Based on the scheme of Carney et al. $(2016), \mathrm{C}=$ confused. ${ }^{(c)}$ Final classification, PS? = potentially pre-stellar, $\mathrm{PDR}=$ narrow, bright ${ }^{12} \mathrm{CO} J=10-9$ emission consistent with a photon-dominated region. ${ }^{(d)}$ See Appendix $\mathrm{C}$ for more details. 


\section{Appendix B: Observation IDs}

This section presents the Herschel observation ID numbers for all WILL HIFI and PACS spectral observations (Table B.1), as well as those of the Herschel PACS and SPIRE photometric maps used to extract source photometry (Table B.2).

Table B.1. Herschel observation identification numbers for WILL HIFI and PACS observations.

\begin{tabular}{|c|c|c|c|c|c|c|}
\hline Name & $\mathrm{H}_{2} \mathrm{O} 1_{10}-1_{01}{ }^{a}$ & $\mathrm{H}_{2} \mathrm{O} 3_{12}-2_{21}^{b}$ & $\mathrm{H}_{2} \mathrm{O} 1_{11}-0_{00}{ }^{c}$ & $\mathrm{H}_{2} \mathrm{O} 2_{02}-1_{11}^{d}$ & PACS 1 & PACS 2 \\
\hline AQU 01 & 1342268681 & 1342268121 & 1342266489 & 1342268160 & 1342254232 & 1342254233 \\
\hline AQU 02 & 1342268682 & 1342268125 & 1342266488 & 1342268159 & 1342254270 & 1342254271 \\
\hline AQU 03 & 1342268683 & 1342268486 & 1342266501 & 1342268154 & 1342254226 & 1342254227 \\
\hline AQU 04 & 1342268684 & 1342268492 & 1342266499 & 1342268156 & 1342254225 & 1342254224 \\
\hline AQU 05 & 1342268462 & 1342268119 & 1342266500 & 1342268155 & 1342254229 & 1342254228 \\
\hline AQU 06 & 1342268463 & 1342268123 & 1342266498 & 1342268153 & 1342254272 & 1342254273 \\
\hline CHA 01 & 1342263152 & 1342254889 & 1342263403 & 1342257661 & 1342267618 & 1342267619 \\
\hline CHA 02 & 1342263153 & 1342266398 & 1342263404 & 1342267974 & 1342265695 & 1342265694 \\
\hline CRA 01 & 1342254318 & 1342254375 & 1342254377 & 1342254338 & 1342254254 & 1342254253 \\
\hline OPH 01 & 1342263422 & 1342263173 & 1342266509 & 1342266759 & 1342266926 & 1342266925 \\
\hline OPH 02 & 1342266423 & 1342263172 & 1342266508 & 1342266758 & 1342263470 & 1342263469 \\
\hline PER 01 & 1342263524 & 1342262778 & 1342263321 & 1342262807 & 1342263509 & 1342263508 \\
\hline PER 02 & 1342263526 & 1342262779 & 1342263322 & 1342262806 & 1342263507 & 1342263506 \\
\hline PER 03 & 1342263525 & 1342262777 & 1342263323 & 1342262808 & 1342263511 & 1342263510 \\
\hline PER 04 & 1342263523 & 1342262776 & 1342263324 & 1342262809 & 1342264251 & 1342264250 \\
\hline PER 05 & 1342263529 & 1342262784 & 1342263325 & 1342262795 & 1342264249 & 13422 \\
\hline PER 06 & 1342263530 & 1342262783 & 1342263326 & 1342262794 & 1342264246 & 1342264247 \\
\hline PER 07 & 1342263531 & 1342262782 & 1342263327 & 1342262802 & 1342264244 & 1342264245 \\
\hline PER 08 & 1342263535 & 1342262786 & 1342263328 & 1342262793 & 1342264242 & 1342264243 \\
\hline PER 09 & 1342263431 & 1342262787 & 1342263329 & 1342262792 & 1342267611 & 1342267612 \\
\hline PER 10 & 1342263534 & 1342262785 & 1342263330 & 1342262791 & 1342267615 & 1342267616 \\
\hline PER 11 & 1342263433 & 1342262780 & 1342263331 & 1342262803 & 1342267607 & 1342267608 \\
\hline PER 12 & 1342263532 & 1342262781 & 1342263332 & 1342262804 & 1342267609 & 1342267610 \\
\hline PER 13 & 1342263536 & 1342262788 & 1342263333 & 1342262790 & 1342267613 & 13422 \\
\hline PER 14 & 1342263537 & 1342262774 & 1342263334 & 1342266763 & 1342263512 & 1342263513 \\
\hline PER 15 & 1342263434 & 1342262775 & 1342263335 & 1342262810 & 1342263514 & 1342263515 \\
\hline PER 16 & 1342263538 & 1342263161 & 1342263336 & 1342266764 & 1342265447 & 1342265448 \\
\hline PER 17 & 1342263539 & 1342263163 & 1342263337 & 1342266765 & 1342263486 & 1342263487 \\
\hline PER 18 & 1342263540 & 1342263162 & 1342263338 & 1342266766 & 1342265449 & 1342265450 \\
\hline PER 19 & 1342263541 & 1342263164 & 1342263339 & 1342266767 & 1342265451 & 1342265452 \\
\hline PER 20 & 1342263542 & 1342263165 & 1342263340 & 1342266768 & 1342265453 & 1342265454 \\
\hline PER 21 & 1342263543 & 1342263166 & 1342263341 & 1342266769 & 1342265455 & 1342265456 \\
\hline PER 22 & 1342263544 & 1342263167 & 1342263342 & 1342266770 & 1342265701 & 1342265702 \\
\hline SCO 01 & 1342266428 & 1342263174 & 1342263319 & 1342266760 & 1342267175 & 1342267176 \\
\hline SERS 01 & 1342268464 & 1342268487 & 1342266502 & 1342268158 & 1342254231 & 1342254230 \\
\hline SERS 02 & 1342268465 & 1342268118 & 1342266497 & 1342268157 & 1342254223 & 1342254222 \\
\hline TAU 01 & 1342266913 & 1342268127 & 1342266486 & 1342266771 & 1342265458 & 1342265457 \\
\hline TAU 02 & 1342266931 & 1342268128 & 1342266485 & 1342266772 & 1342265460 & 1342265459 \\
\hline TAU 03 & 1342266932 & 1342268129 & 1342266484 & 1342266773 & 1342265462 & 1342265461 \\
\hline TAU 04 & 1342266933 & 1342268131 & 1342266482 & 1342266774 & 1342265464 & 1342265463 \\
\hline TAU 06 & 1342266934 & 1342268130 & 1342266483 & 1342266775 & 1342265465 & 1342265466 \\
\hline TAU 07 & 1342266935 & 1342268133 & 1342266481 & 1342266776 & 1342265467 & 1342265468 \\
\hline TAU 08 & 1342266930 & 1342268135 & 1342266477 & 1342266778 & $-^{e}$ & \\
\hline TAU 09 & 1342266936 & 1342268134 & 1342266478 & 1342268144 & 1342267856 & 1342267857 \\
\hline W40 01 & 1342268272 & 1342268488 & 1342266496 & 1342268152 & 1342254221 & 1342254220 \\
\hline W40 02 & 1342268273 & 1342268122 & 1342266495 & 1342268151 & 1342254269 & 1342254268 \\
\hline W40 03 & 1342268676 & 1342268489 & 1342266494 & 1342268150 & 1342254267 & 1342254266 \\
\hline W40 04 & 1342268677 & 1342268124 & 1342266493 & 1342268149 & 1342254261 & 1342254260 \\
\hline W40 05 & 1342268678 & 1342268490 & 1342266492 & 1342268148 & 1342254265 & 1342254264 \\
\hline W40 06 & 1342268679 & 1342268491 & 1342266491 & 1342268147 & 1342254258 & 1342254259 \\
\hline W40 07 & 1342268680 & 1342268120 & 1342266490 & 1342268146 & 1342254262 & 1342254263 \\
\hline
\end{tabular}

Notes. ${ }^{(a)}$ Observation also contains $\mathrm{H}_{2}{ }^{18} \mathrm{O} 1_{10}-1_{01}$ in the other sideband. ${ }^{(b)}$ Observation also contains $\mathrm{CO} J=10-9$ in the same sideband. (c) Observation also contains $\mathrm{H}_{2}{ }^{18} \mathrm{O} 1_{11}-0_{00}$ in other sideband and ${ }^{13} \mathrm{CO} J=10-9$ in the same sideband. ${ }^{(d)}$ Observation also contains $\mathrm{C}^{18} \mathrm{O}$ $J=9-8$ in the same sideband. ${ }^{(e)}$ Observation scheduled but not successfully observed before the end of science operations. 
J. C. Mottram et al.: Outflows, infall and evolution of a sample of embedded low-mass protostars

Table B.2. Herschel observation identification numbers for continuum PACS and SPIRE observations used to determine far-IR SED fluxes.

\begin{tabular}{|c|c|c|c|c|}
\hline Region & PACS $70 \mu \mathrm{m}$ & PACS $100 \mu \mathrm{m}$ & PACS $160 \mu \mathrm{m}$ & SPIRE 250,350 and $500 \mu \mathrm{m}$ \\
\hline Aquila, Serpens South \& W40 & 1342186277,1342186278 & 1342193534,1342193535 & 1342193534,1342193535 & 1342186277,1342186278 \\
\hline \multirow[t]{2}{*}{ Chameleon } & 1342213178,1342213179 & 1342224782,1342224783 & 1342224782,1342224783 & 1342213178,1342213179 \\
\hline & 1342213180,1342213181 & 1342212708,1342212709 & 1342212708,1342212709 & 1342213180,1342213181 \\
\hline Corona Australis & 1342206677,1342206678 & 1342218806,1342218807 & 1342218806,1342218807 & 1342206677,1342206678 \\
\hline \multirow[t]{2}{*}{ Ophiuchus } & 1342205093,1342205094 & 1342227148,1342227149 & 1342227148,1342227149 & 1342205093,1342205094 \\
\hline & & 1342227148,1342227149 & 1342227148,1342227149 & \\
\hline \multirow[t]{2}{*}{ Perseus } & 1342190326,1342190327 & 1342227103,1342227104 & 1342227103,1342227104 & 1342190326,1342190327 \\
\hline & 1342214504,1342214505 & 1342216077,1342216078 & 1342216077,1342216078 & 1342214504,1342214505 \\
\hline Scorpius & 1342267724,1342267725 & - & 1342267724,1342267725 & \\
\hline \multirow[t]{3}{*}{ Taurus } & 1342190616,1342190654 & 1342228005,1342228006 & 1342190616,1342228005 & 1342190616,1342190652 \\
\hline & 1342190655 & & 1342228006,1342190652 & 1342190653,1342190654 \\
\hline & & & 1342190653 & $\begin{array}{c}1342190655,1342202253 \\
1342202254\end{array}$ \\
\hline
\end{tabular}

\section{Appendix C: Discussion of individual cases}

This section presents notes on individual sources to explain oddities in the data that bear specific mention.

AQU 01: the additional emission component observed only in the $\mathrm{H}_{2} \mathrm{O} 1_{10}-1_{01}$ line towards this source (which has the largest beam) is almost certainly due to another source on the edge of the beam. This source is outside the beam for all other HIFI observations.

CHA 01: this source shows a relatively small and weak but detectible outflow in $\mathrm{CO} J=3-2$, as well as a relatively narrow detection in $\mathrm{H}_{2} \mathrm{O}$ with $\mathrm{HIFI}$ and detections in [O I], $\mathrm{H}_{2} \mathrm{O}$, $\mathrm{CO}$ and $\mathrm{OH}$ with PACS. The principle reason this source is not designated as a Class $\mathrm{I}$ is the non-detection of $\mathrm{HCO}^{+}$. The low velocity of the outflow suggests this may either be a remnant from the Class I phase, or a disk-wind such as recently seen by ALMA in HD 163296 (Klaassen et al. 2013).

CHA 02: the non-detection of water emission in the HIFI observations for this Class I source is unsurprising given that only a few lines are marginally detected in PACS and no outflow is detected in $\mathrm{CO} J=3-2$. There is a detection in [O I], suggesting that some form of jet or wind is present. This source is therefore probably nearing the Class II phase and simply has too tenuous an envelope for a significant outflow component to still be present and detectible.

OPH 01: this source does not show compact $\mathrm{HCO}^{+}$or $\mathrm{C}^{18} \mathrm{O}$ emission, so is not Class 0/I. There is a cold, starless core to the north that increasingly dominates at longer wavelengths (e.g. Sadavoy et al. 2010) and is causing the source to appear more embedded than it really is. The strong, narrow $\mathrm{CO} J=$ 10-9 emission profile, along with the PACS detection of $\mathrm{CO}$ $J=16-15$ are most likely from a PDR or bow-shock, possibly caused by interaction between the infrared source and the cold core if they are spatially associated. Given that the primary infrared source is visible in the mid-IR (e.g. Brown et al. 2013), we classify it as Class II, whilst noting that there is also PDR-like emission.

PER 02: high-resolution BIMA observations of the $\mathrm{CO}$ outflows and Spitzer IRAC scattered light observations show that the blue outflow lobe of this source is contaminated by the blue outflow lobe originating from L1448-MM to the south (Kwon et al. 2006; Tobin et al. 2007). We therefore do not report outflow properties as it is impossible to disentangle the two flows. However, the BIMA observations do show activity related to the targeted source, so it is still classified as having a detected outflow for the purposes of source evolution. The broad absorption in the blue outflow lobe in the ground-state water lines likely takes place against the outflow from L1448-MM, indicating that it is between this source and the observer. In particular, the saturated absorption feature below the continuum in $\mathrm{H}_{2} \mathrm{O} 1_{11}-0_{00}$ (see San José-García 2015) rules out the possibility that this is instead caused by a combination of emission from the different outflows in the beam. Thus, the source is most likely further away than the outflow from L1448-MM and the two are probably not interacting directly.

PER 04: the non-detection of water for this Class 0 source is slightly surprising given the detection of an outflow in $\mathrm{CO}$ $J=3-2$. However, the low velocity of the outflow may mean that any shocks are not fast enough to sputter water from the grains or warm enough to lead to efficient gas-phase formation. This may indicate that this source is particularly young or has been in a lower-accretion phase for some time.

PER 06: while the CO $J=3-2$ data are consistent with an outflow originating from this source (NGC 1333-IRAS2B), it is impossible to disentangle the contribution of NGC 1333IRAS2A, something even Plunkett et al. (2013) find impossible at three times higher spatial resolution with CARMA, so we follow those authors in not quoting any outflow property values.

PER 08: at least part of the strange outflow morphology from this source may be from another nearby source or indicate that it is a multiple. However, it is a single star in high-resolution VLA observations (Tobin et al. 2016) and there are no other obvious infrared candidates nearby so for now we attribute all the outflow emission to this protostar.

PER 12: the red outflow lobe of NGC 1333-IRAS4A passes through the $\mathrm{H}_{2} \mathrm{O} 1_{10}-1_{01}$ beam but not the other transitions that have smaller beam-sizes; hence the detection in only this transition. There is no evidence in the CO $J=3-2$ data of an outflow associated with this source. However, Tobin et al. (2015) note that Spitzer images are suggestive of a jet or outflow related to the source (see their Fig. 19), so the non-detection is likely because the outflow is in the plane of the sky rather than because this source does not have an outflow.

PER 22: the morphology of the outflow in CO $J=3-2$ is suggestive of two outflows, particularly in the red lobe, with one being approximately north-south and the other east-west. Enoch et al. (2009) find another, more evolved Class I source (Per-emb 55) 9" away, which therefore lies within the Herschel 
beam. VLA observations resolve this additional source into a binary (Tobin et al. 2016), suggesting that this is actually a triple if Per-emb 8 and 55 are spatially associated.

SERS 01: this source is significantly offset in both the continuum and molecular lines from the position given by Maury et al. (2011), which is likely why it is relatively weak in the HIFI and PACS observations.

TAU 03: most of the outflow near this source is likely due to a neighbouring, probably younger, source, though there does seem to be a weak flow from the primary target as well.

TAU 06: the outflow observed in CO $J=3-2$ seems to have two blue outflow lobes, and the red and blue outflow lobes are not well aligned. Given the low velocity of the outflow, it may be close to the plane of the sky, or there may be multiple outflows in the region. There is no other obvious driving source in the vicinity however, so we assign all the emission to the target.

TAU 07-09: these Class II sources exhibit some line broadening in CO $J=3-2$ around the source position. While the linewings are not at high enough velocity offset $\left(F W Z I \lesssim 10 \mathrm{~km} \mathrm{~s}^{-1}\right)$ to be considered related to a true outflow, this is suggestive of either higher turbulence or a small disk wind (cf. Klaassen et al. 2013) in these sources.

W40 01: the HIFI $\mathrm{H}_{2} \mathrm{O}$ spectra show a broad line-wing, in addition to PDR-like absorption close to the $v_{\mathrm{LSR}}$, consistent with an outflow, though there is no outflow detected in low- $J$
CO.The source is compact and relatively bright at $70 \mu \mathrm{m}$, which led Könyves et al. (2015) to designate this source as protostellar, rather than prestellar, and it is detected in the mid-IR. It is therefore most likely a Class 0 source, though the presence of the W40 PDR complicates the detection and passage of the outflow.

W40 03-06: these sources show little or only very weak continuum emission at wavelengths shorter than $100 \mu \mathrm{m}$, while at longer wavelengths, the emission is not particularly compact. They also do not show any signs of outflow and the only line detections are related to emission from the W40 PDR. We therefore designate these sources as potentially prestellar, but note that the PDR makes a reliable classification more difficult.

W40 07: the outflow observed towards this source in $\mathrm{CO}$ $J=3-2$ is surprisingly strong given that there is no emission detected in $\mathrm{H}_{2} \mathrm{O}$ and only a faint, narrow and tentative detection in CO $J=10-9$. The low fluxes in the mid-IR would seem to suggest that there is little warm gas around this source, but the sub-mm and $\mathrm{mm}$ continuum detections indicate a significant reservoir of cold dust. The bright and compact nature of the $70 \mu \mathrm{m}$ PACS continuum emission and shape of the SED led Könyves et al. (2015) to designate this source as protostellar. It is therefore most likely a very young protostellar source where the outflow has not become fast and warm enough to be detected in $\mathrm{H}_{2} \mathrm{O}$ and high- $J$ CO. 